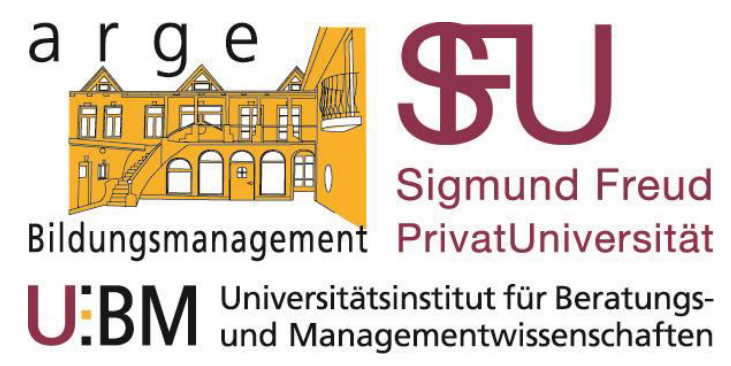

Masterarbeit

\title{
Die österreichische Erinnerungs- und Gedenkkultur am Beispiel des Nationalfonds der Republik Österreich für Opfer des Nationalsozialismus - Gesetzlicher Auftrag und soziale Verantwortung
}

\author{
Verfasserin \\ Stephanie Mayerhofer, BA \\ Zur Erlangung des akademischen Grades \\ Master of Arts - \\ M.A. (Sozialpädagogik und Sozialmanagement)
}

Wien, im September 2019

Fakultät für Psychologie der Sigmund Freud Privat Universität Wien

Institut ARGE Bildungsmanagement

Studienrichtung: Universitätslehrgang

Beratungswissenschaften und Management sozialer Systeme -

Studienschwerpunkt:

Sozialpädagogik und Sozialmanagement

Begutachter: $\quad$ FH-Prof. apl. Prof. Dr. rer. soc. Dr. phil. habil. Bernhard Plé 


\section{Abstract}

1995 wurde der Nationalfonds der Republik Österreich für Opfer des Nationalsozialismus [im Folgenden Nationalfonds genannt, Anm. d. Verf.] gegründet. Der Fonds hat die Aufgabe Entschädigungsmaßnahmen an Opfer des Nationalsozialismus, die bis dahin unzureichend erfolgten, zu erbringen und steht für die Verantwortungsübernahme der Republik Österreich gegenüber den Opfern des Nationalsozialismus. Die Einbettung des Nationalfonds und dessen Leistungen für die österreichische Erinnerungskultur, die zukünftigen Aufgaben, die in der letzten Novelle des Nationalfondsgesetzes aus dem Jahr 2017 verankert wurden und die damit zusammenhängenden strukturellen Veränderungen in der Organisation werden in der vorliegenden Arbeit theoretisch ausgearbeitet und empirisch durch qualitative Forschung untersucht. Anhand leitfadengestützter ExpertInneninterviews und deren Auswertung durch qualitative Inhalts- und Themenanalyse wird aufgezeigt, dass der Nationalfonds durch seine Definition des Opferbegriffs und der Projektförderung zu einer Bewusstseinsbildung in der Zivilgesellschaft und zu einer Erweiterung und einem Paradigmenwechsel in der Erinnerungskultur beigetragen hat. Kritik an einer Institutionalisierung und Ritualisierung der Erinnerungskultur sowie der erneute Generationenwechsel verdeutlichen die Notwendigkeit einer Aktualisierung derselben. Der Nationalfonds kann durch seine Entwicklung von einer Auszahlungs- und Förderstelle hin zu einer Vermittlungsstelle für Wissenschaft, Beratung und Aufarbeitung den geforderten aktuellen Bezugsrahmen schaffen und hinkünftig als Koordinierungsstelle für staatliches Erinnern und Gedenken fungieren. Zusätzlich dazu kann der Nationalfonds als gesellschafts- und bildungspolitische Instanz mit der notwendigen niederschwelligen Herangehensweise auch neue Zielgruppen der heutigen heterogeneren Gesellschaft erreichen und so zu einer zukünftigen Erinnerungskultur, die sich nicht nur auf die Geschichte des Holocaust bezieht, sondern auch interkulturelles Verständnis fördert und gegenwärtigen gesellschaftlichen Ausgrenzungstendenzen entgegenwirkt, beitragen. 
„[...] Only in remembering what happened to us can the world assure that it will not happen to others."

(Elie Wiesel, 1980) 


\section{Inhaltsverzeichnis}

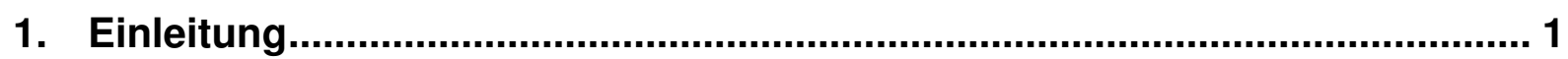

2. Erinnerungs- und Gedenkkultur in Österreich .......................................... 2

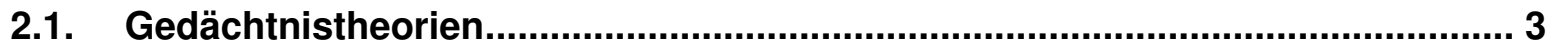

2.2. Das Trauma der Überlebenden......................................................................... 7

2.3. Die Überlebensschuld.....................................................................................10

2.4. Die transgenerationale Weitergabe von Traumata..............................................12

2.5. Österreich und die Opferthese ....................................................................15

2.6. Von der Vergangenheitsbewältigung zur Erinnerungskultur............................20

2.7. Opferidentifizierung und Opferorientierung ......................................................23

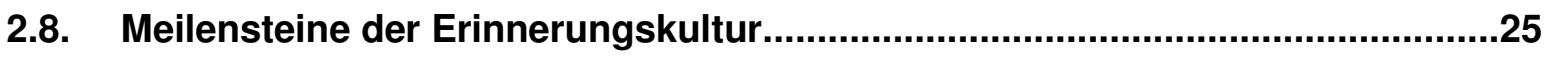

2.8.1. Die Fernsehserie „Holocaust - Die Geschichte der Familie Weiss“ ............................. 25

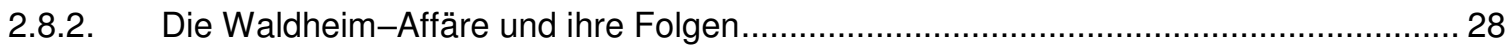

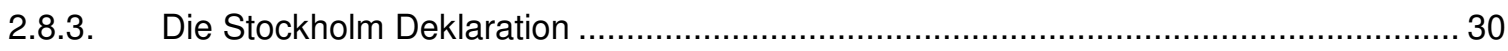

2.8.4. International Holocaust Remembrance Alliance (IHRA) …………………………..... 34

2.9. Kritik zu Erinnerungs- und Gedenkkultur.........................................................36

2.10. Die Zukunft der Erinnerungskultur............................................................39

3. Nationalfonds der Republik Österreich für Opfer des Nationalsozialismus...

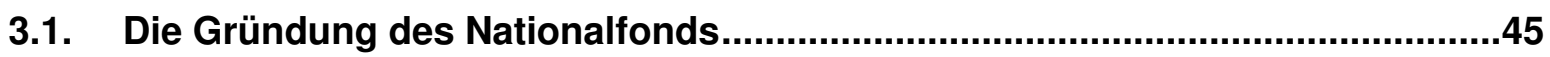

3.2. Die organisatorische Struktur des Nationalfonds..........................................49

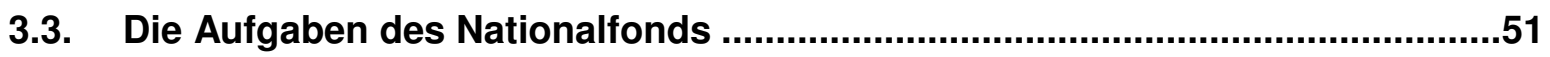

3.4. Allgemeiner Entschädigungsfonds für Opfer des Nationalsozialismus...........60

3.5. Fonds zur Instandsetzung der jüdischen Friedhöfe in Österreich ....................64

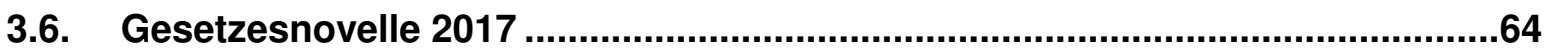

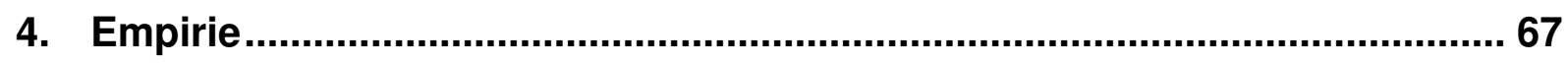

4.1. Definition des Untersuchungsgegenstandes ...............................................67

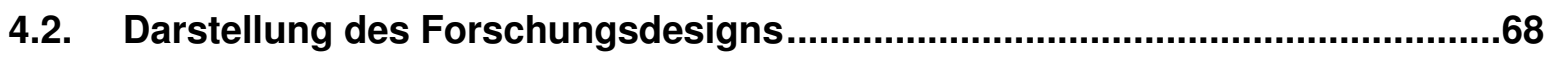

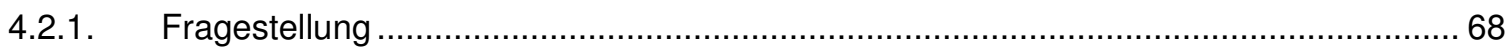

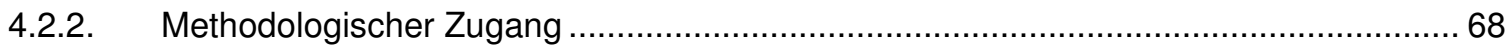




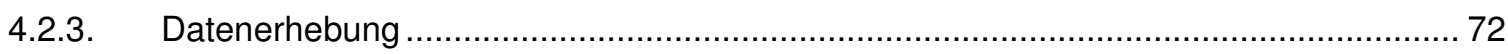

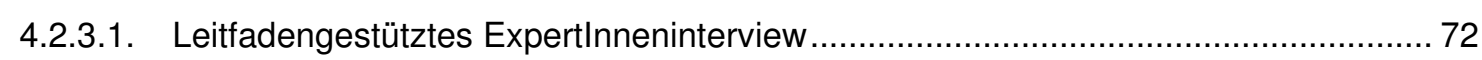

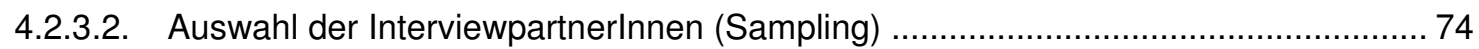

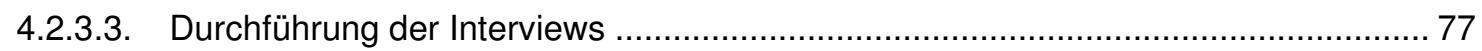

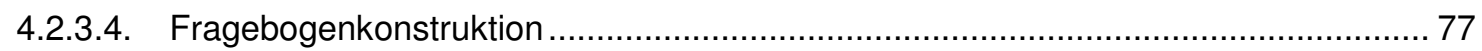

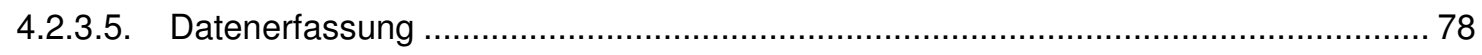

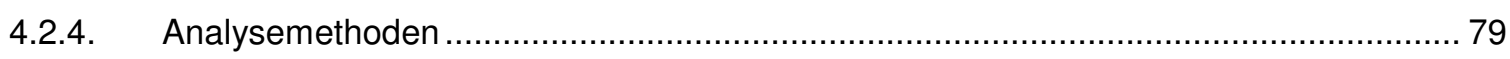

4.2.4.1. Zusammenfassende qualitative Inhaltsanalyse ........................................... 79

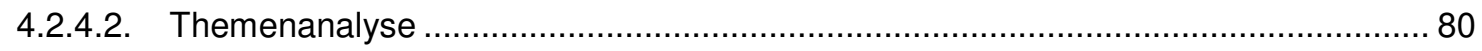

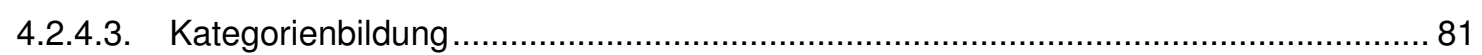

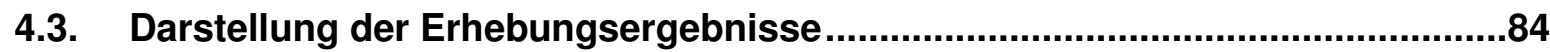

4.3.1. Kategorie 1: Organisation, Struktur und Wandel des Nationalfonds .......................... 84

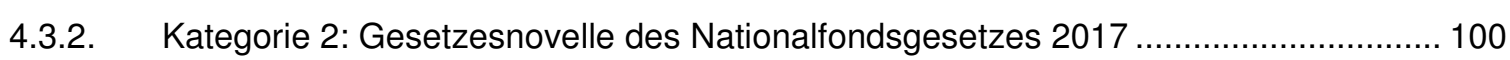

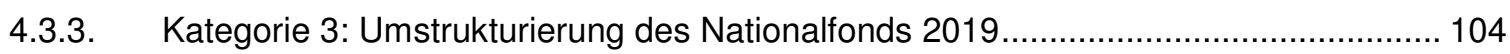

4.3.4. Kategorie 4: Herausforderungen für den Nationalfonds ........................................ 111

4.3.5. Kategorie 5: Zukunftsperspektiven für den Nationalfonds..................................... 121

4.3.6. Kategorie 6: Meilensteine in der Erinnerungskultur.................................... 128

4.3.7. Kategorie 7: Erinnerungskultur - Wandel und Funktion ......................................... 132

4.3.8. Kategorie 8: Kritik an Erinnerungskultur .................................................... 141

4.3.9. Kategorie 9: Herausforderungen und Zukunftsperspektiven für die Erinnerungskultur ....

5. Conclusio und kritische Reflexion ........................................................ 150

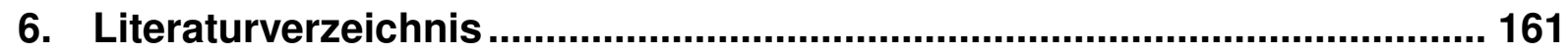

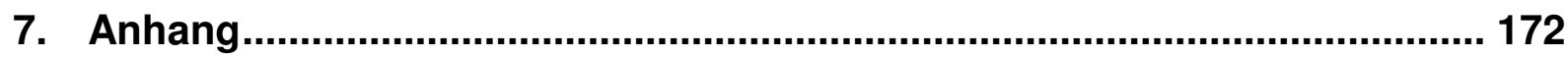

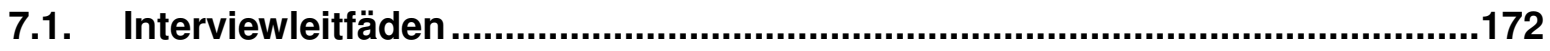

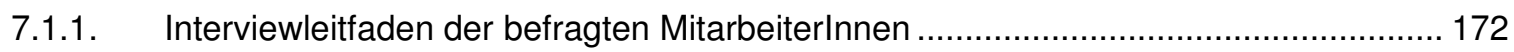

7.1.2. Interviewleitfaden der befragten internen Expertinnen ........................................ 173

7.1.3. Interviewleitfaden der befragten externen Expertin (Dr. Janistyn-Novák) ................... 174

7.1.4. Interviewleitfaden der befragten externen Expertin (Mag. Maschke) ....................... 175

7.1.5. Interviewleitfaden der befragten externen Expertin (Dr. Uhl) ............................. 176

7.2. Extraktionstabellen ........................................................................................177

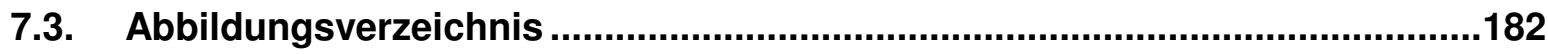

8. Eigenständigkeitserklärung …........................................................... 183 


\section{Einleitung}

„[...] Only in remembering what happened to us can the world assure that it will not happen to others." ${ }^{1}$ - Nur durch die Erinnerung an das, was uns widerfahren ist, kann sichergestellt werden, dass es anderen nicht widerfährt - so Elie Wiesel, ein Überlebender des Holocaust, in einem Statement, das während einer Pressekonferenz 1980 in New York anlässlich der Ankündigung des ,World Gathering of Holocaust Survivors' in Israel im Jahr 1981 verlesen wurde.

Gedenktage, Gedächtnisorte, Gedenkstätten, zahlreiche Filme, Bücher und Publikationen - die Landschaft der heutigen Erinnerungs- und Gedenkkultur zum Holocaust ist vielfältig. Phrasen wie ,Nie wieder!' sind geläufig. Schlagworte wie kollektives Gedächtnis, kosmopolitisches Gedächtnis und Erinnerungskultur finden sich in wissenschaftlichen Publikationen. Doch wie findet die Auseinandersetzung mit dem Erinnern, dem Nicht-Vergessen jetzt und vor allem in der Zukunft statt? Wie können die Erinnerungen bewahrt werden, wenn keine ZeitzeugInnen mehr am Leben sein werden, um darüber zu berichten was innen widerfahren ist? Wie können Erinnerungen an die nächsten Generationen weitergegeben werden? Mit diesen Fragen beschäftigt sich der Nationalfonds der Republik Österreich für Opfer des Nationalsozialismus. Die vorliegende Arbeit setzt sich mit der Geschichte der Erinnerungs- und Gedenkkultur in Österreich und des Nationalfonds auseinander, um die Frage zu beantworten, welchen Beitrag der Nationalfonds für die Erinnerungskultur leistet, insbesondere in Hinblick auf die gesetzlich erweiterten Aufgabenbereiche des Fonds. Es wurde folgende Forschungsfrage gestellt:

Was kann der Nationalfonds aufgrund der gesetzlichen Erweiterung der Aufgabenbereiche für die österreichische Erinnerungs- und Gedenkkultur leisten und welche organisatorischen Herausforderungen ergeben sich dadurch?

Anhand dieser Fragestellung soll aufgezeigt werden, welche Aufgaben der Nationalfonds im Laufe seines Bestehens übernommen hat, welche Auswirkungen die gesetzliche Erweiterung der Aufgaben für die Organisation hat und welche

${ }^{1}$ http://pdfs.jta.org/1980/1980-02-14_032.pdf?_ga=2.125613514.1572620604.1566027527-

1448551427.1546774246 [Datum des Zugriffs: 30.8.2019] 
Zukunftsperspektiven für die Erinnerungs- und Gedenkkultur im Rahmen der Tätigkeit des Nationalfonds bestehen. Um die Forschungsfrage zu beantworten, werden die folgenden Themenbereiche theoretisch beschrieben: Die Entwicklung der österreichischen Erinnerungs- und Gedenkkultur aus der Vergangenheitsbewältigung wird skizziert. Begriffe wie die posttraumatische Belastungsstörung bei Überlebenden des Holocaust, die transgenerationale Weitergabe von Traumata, sowie die Überlebensschuld, Opferorientierung und Opferidentifizierung werden definiert und erläutert. Des Weiteren werden die österreichische Opferthese und die zentralen Meilensteine in der Entwicklung der Erinnerungskultur beschrieben. Ebenso werden die Gründung des Nationalfonds und dessen Aufgabengebiete erklärt und, um einen vollständigen Überblick zu erhalten, auch auf die Arbeit des Allgemeinen Entschädigungsfonds für Opfer des Nationalsozialismus und des Fonds zur Instandsetzung der jüdischen Friedhöfe in Österreich eingegangen. Besonderes Augenmerk wird auf die Beschreibung der letzten Gesetzesnovelle des Nationalfondsgesetzes gelegt, die zu einer Erweiterung der Aufgaben führte. Im empirischen Teil der Arbeit wird detaillierter auf den Forschungsgegenstand eingegangen und das Design der Methodik sowie die verwendeten Analysemethoden dargestellt. Anschließend werden die Ergebnisse präsentiert, um in der Conclusio übersichtlich dargestellt, kritisch reflektiert und mit den theoretischen Ausführungen in Bezug gesetzt zu werden.

\section{Erinnerungs- und Gedenkkultur in Österreich}

Um den Begriff der Erinnerungskultur erklären und verstehen zu können, muss zuerst auf die Entstehungsgeschichte dieser Wortschöpfung eingegangen werden. Aleida Assmann zufolge handelt es sich um eine neue Wortkreation, die sich in den 1990er Jahren etabliert hat (vgl. Assmann, 2016, S.29). Welche Umstände zum Entstehen der Erinnerungskultur, wie man sie heute versteht, in Österreich führten, wird in den folgenden Kapiteln erläutert. Zwei Faktoren trugen wesentlich zum heutigen Verständnis von Erinnerungskultur bei. In der Nachkriegszeit herrschte Aufbruchsstimmung, man wollte sich nicht mehr mit der Vergangenheit beschäftigen. Die österreichische Regierung und die Gesellschaft blickten nach vorne. In diesem Zusammenhang wird in der Arbeit auf die Haltung des offiziellen Österreich eingegangen: die sogenannte Opferthese und der lange Weg von der 
Vergangenheitsbewältigung hin zu einer Erinnerungskultur werden erläutert. Zwecks Vollständigkeit wird auf einen zweiten Faktor eingegangen: die späte Anerkennung der Opfer des nationalsozialistischen Regimes. Den Überlebenden des Holocaust wurde in der ersten Nachkriegszeit jegliche Anerkennung verwehrt. Es dauerte Jahrzehnte, bis mit einer wissenschaftlichen Aufarbeitung der gesellschaftlichen, psychosozialen und traumatischen Folgen des Holocaust für die Überlebenden begonnen wurde. Im Rahmen dessen wird auch auf die Klassifizierung der posttraumatischen Belastungsstörung und die transgenerationale Weitergabe traumatischer Erfahrungen eingegangen. Um ein vollständigeres Bild des Begriffs und der Bedeutung von Erinnerungskultur und Gedenkkultur in Österreich zu geben, werden die oben erwähnten Faktoren in den folgenden Kapiteln beschrieben.

\subsection{Gedächtnistheorien}

Um die Entstehung der Erinnerungskultur beschreiben zu können, werden Ansätze aus der Gedächtnistheorie verwendet. Erinnerungskultur basiert auf gemeinsamen Erinnerungen einer Gesellschaft. Der Soziologe Maurice Halbwachs beschäftigte sich in den 1920er Jahren mit der Erforschung des sozialen Gedächtnisses (vgl. Assmann, 2018, S.25). 1925 erschien sein Buch ,Das Gedächtnis und seine sozialen Bedingungen', welches heute als Standardwerk zur Gedächtnisforschung gilt. Laut Halbwachs beruht das Gedächtnis auf sozialen Interaktionen. Sprache und Kommunikation stellen für inn eine Bedingung für kollektives Gedächtnis und Denken dar (vgl. Halbwachs, 1985, S.368). Er betont damit den gesellschaftlichen Rahmen des Gedächtnisses und verbindet Erinnerungen mit Kommunikation.

Für Halbwachs existiert „[...] kein mögliches Gedächtnis außerhalb derjenigen Bezugsrahmen, deren sich die in der Gesellschaft lebenden Menschen bedienen, um ihre Erinnerungen zu fixieren und wiederzufinden." (Halbwachs, 1985, S.121)

Soziale Gefüge sind für die Schaffung kollektiver Erinnerung unerlässlich. Halbwachs zieht Rückschlüsse auf das kollektive Gedächtnis durch die Beschreibung von unterschiedlichen gesellschaftlichen Gruppen (wie z.B. Familien, religiösen Gruppen, gesellschaftlichen Klassen etc.). Durch die Zugehörigkeit zu einer Gruppe würden gemeinsame, kollektive Erinnerungen entstehen. 
Halbwachs schließt seine Überlegungen wie folgt ab:

Daraus geht hervor, daß das gesellschaftliche Denken wesentlich ein Gedächtnis ist, und daß dessen ganzer Inhalt nur aus kollektiven Erinnerungen besteht, daß aber nur diejenigen von innen und nur das an innen bleibt, was die Gesellschaft in jeder Epoche mit ihren gegenwärtigen Bezugsrahmen rekonstruieren kann. (Halbwachs, 1985, S.390)

Assmann sieht im kollektiven Gedächtnis eine doppelte Bedeutung: „Es repräsentiert einen als zentral bewerteten Ausschnitt der Vergangenheit und ist repräsentativ für Einzelschicksale. [...] Ein kollektives Gedächtnis ermöglicht es den Mitgliedern einer Gesellschaft, über räumliche und zeitliche Entfernungen hinweg Bezugspunkte in der Vergangenheit festzuhalten und gemeinsame Orientierungsformen aufzubauen." (Assmann, 2016a, S.17)

Der Historiker Reinhart Koselleck wiederum geht davon aus, dass Erinnerungen nur durch eigene Erfahrungen entstehen können. Koselleck erweitert Halbwachs' Ansatz mit einem Bezugsrahmen für kollektive Erinnerungen, dem Konzept der „[...] kollektive[n] Bedingungen [...]." (ebd., S.18) Er unterscheidet somit zwischen persönlichen und kollektiven Erinnerungen und verdeutlicht damit ,[...] die Existenz des Phänomens der kollektiven Erinnerung. [...] wenn auch auf einer anderen Ebene: der Ebene des Gedenkens.“ (ebd., S.19)

Dieses Phänomen der kollektiven Erinnerung zeigt sich vor allem im Gedenken. Gedenktage und Gedenkorte sind kollektiv im Gedächtnis von Personengruppen, Staaten oder gar weltweit verankert. Assmann betont in diesem Zusammenhang auch die Freiwilligkeit der Teilnahme am kollektiven Erinnern (vgl. ebd.).

Laut Assmann wird das kollektive Gedächtnis durch Politik bestimmt. „In totalitären Gesellschaften ist es der Staat, der das kollektive Gedächtnis schafft und kontrolliert; in Demokratien sind es obendrein auch die Bürger, die Künstler, die Parteien und vor allem die Medien." (ebd., S.21) Das nationale Gedächtnis, wie es Assmann 
formuliert, bezeichnet eine Form des politischen Gedächtnisses, welches „[...] zusammen mit starken Loyalitätsbindungen auch starke vereinheitlichte Wir-Identität hervorbringt." (Assmann, 2018, S.36)

Durch kollektive Erinnerungen werden Rahmen geschaffen, die Identität erzeugen und „[...] die Brücke zwischen Vergangenheit, Gegenwart und Zukunft [...]" (Assmann, 2016a, S.29) bilden. Die Sprache, wie es auch bereits Halbwachs beschrieben hat, ermöglicht erst „[...] das ganze System der damit verbundenen gesellschaftlichen Konventionen, die uns jederzeit die Rekonstruktion unserer Vergangenheit gestattet." (Halbwachs, 1985, S.369)

Da gesellschaftliche Gefüge jedoch zeitlich dynamisch sind, ändern sich auch die Bezugsrahmen der kollektiven Erinnerungen mit der Zeit. Mit der Rekonstruktion von Vergangenheit kann diese auch verformt werden (vgl. ebd. S.381). Halbwachs verbindet den Gedanken der Vernunft mit dem Gedächtnis. Nicht nur Rekonstruktion, sondern auch Modifizierung der Vergangenheit beruht auf der Vernunft. Er fasst dies wie folgt zusammen: „[...] die sozialen Überzeugungen [...] sind kollektive Traditionen oder Erinnerungen, aber sie sind zugleich auch Ideen oder Konventionen, die aus der Kenntnis des Gegenwärtigen entspringen." (ebd. S.389) Alte Überzeugungen können in einer Gesellschaft aufgegeben werden, wenn neue gefunden werden (vgl. ebd. S.388).

Erinnerungen unterliegen einem gewissen Zeitfenster. Assmann sieht dieses Zeitfenster im Generationenwechsel:

Nach achtzig bis hundert Jahren kommt es zu einem deutlichen Einschnitt. Das ist die Periode, in der verschiedene Generationen - in der Regel sind es drei, im Grenzfall sogar fünf - gleichzeitig existieren und durch persönlichen Austausch eine Erfahrungs-, Erinnerungs- und Erzählgemeinschaft bilden. (Assmann, 2018, S.25) 
Individuelle Erinnerungen unterliegen diesem Generationenwechsel. Jede Generation gestaltet ihre eigenen Zugänge zur Vergangenheit und lässt sich nicht durch frühere Generationen beeinflussen, was zu Konflikten führen kann (vgl. Assmann, 2018, S.27). Auch Halbwachs attestiert dem kollektiven Gedächtnis räumliche und zeitliche Grenzen, wie bereits in Hinblick auf die Gedächtnisrahmen erläutert wurde.

Ein weiterer Ansatz aus der Gedächtnistheorie ist der des kulturellen Gedächtnisses. Das kulturelle Gedächtnis wird als eine Erweiterung des kollektiven Gedächtnisses beschrieben. Das kollektive Gedächtnis entsteht durch Kommunikation und Sprache, wie bereits erläutert wurde. Assmann beschreibt die Grundlagen eines kulturellen Gedächtnisses: „Voraussetzung und Fundament eines kulturellen Gedächtnisses ist die Überschreibung von Erinnerungen und Erfahrungen auf materielle Datenträger. Ohne Kodifizierung in Zeichen und Symbolen, ohne Formung in Texten und Bildern gibt es kein kulturelles Gedächtnis." (Assmann, 2018, S.235) Ein kulturelles Gedächtnis entsteht nicht nur durch das Festschreiben und Bewahren von Erinnerungen, sondern „[...] erst durch individuelle Wahrnehmung, Wertschätzung und Aneignung, wie sie durch die Medien, durch kulturelle Einrichtungen und Bildungsinstitutionen vermittelt werden [...]." (ebd., S.57) Damit unterliegt das kulturelle Gedächtnis nicht mehr dem Generationenwechsel und kann ein soziales Langzeitgedächtnis formen, wie es Assmann beschreibt.

Die erläuterten Ansätze zeigen die unterschiedlichen Einflüsse auf die Erinnerungen. Assmann formuliert vier Träger von Gedächtnisformen: „[...] Individuen, soziale Gruppen, politische Kollektive und Kulturen." (Assmann, 2018, S.59) Sie kritisiert in diesem Zusammenhang den Begriff des kollektiven Gedächtnisses als zu vage und schlägt eine Unterscheidung zwischen sozialen, politischen und nationalen Gedächtnissen vor (vgl. ebd., S.60). 


\subsection{Das Trauma der Überlebenden}

Viele Überlebende des Holocaust litten an einer posttraumatischen Belastungsstörung, im englischen PTSD - posttraumatic stress disorder genannt. ${ }^{2}$ Doch die Klassifizierung der traumatischen Folgen für die Überlebenden als Form der posttraumatischen Belastungsstörung sollte erst viel später erfolgen. Der Begriff ,Überlebende" wurde erst ab Ende der 1950er Jahre in wissenschaftlichen Arbeiten eingeführt. Dies erfolgte vorrangig zur psychiatrischen Klassifizierung der Folgen von Deportationen und Qualen der Überlebenden (vgl. Zajde, 2011, S.17). Die Symptome, an denen die Überlebenden der Konzentrationslager litten, wurden unter dem Begriff KZ- oder Überlebenden-Syndrom zusammengefasst (vgl. Zajde, 2011, S.17f). Eine Klassifizierung der Symptome, zu denen intensive Angstgefühle, Vermeidungsmechanismen, emotionale Instabilität, traumatische Erinnerungen und dissoziative Angstzustände gehörten, sollten die Behandlung der Überlebenden erleichtern und innen Anspruch auf finanzielle Entschädigung ermöglichen (vgl. Zajde, 2011, S.18).

1961 wurde vom amerikanischen Psychiater William G. Niederland der Begriff Holocaust-Syndrom geprägt. Er umfasste auch die Überlebenden des nationalsozialistischen Regimes, die nicht in Konzentrations- und Vernichtungslagern interniert gewesen waren (vgl. Vyssoki, Tauber, Strusievici \& Schürmann-Emanuely, 2004, S.200).

1980 wurde die posttraumatische Belastungsstörung in der dritten Auflage des, The Diagnostic and Statistical Manual of Mental Disorders (DSM-III) der American Psychiatric Association festgehalten (vgl. Zajde, 2011, S.24). Seit den 1990er Jahren wird das Überlebenden-Syndrom beziehungsweise das Holocaust-Syndrom als komplexe posttraumatische Belastungsstörung klassifiziert (vgl. Vyssoki et al., 2004, S.197). „Fortan wurden die Kriterien des PTSD auf die Überlebenden der Shoah und folglich auf alle Opfer der NS-Verfolgung angewandt." (Krystal, Yehuda, 1994, zit.n. Vyssoki et al., 2004, S.200) Im Internationalen Krankheitsklassifikationssystem (ICD10) der Weltgesundheitsorganisation (WHO) werden die Symptome unter

\footnotetext{
${ }^{2}$ In diesem Kapitel sind Textteile meiner 2014 an der FH Campus Wien vorgelegten Bachelorarbeit mit dem Titel „Das Holocaust-Syndrom als Form der posttraumatischen Belastungsstörung“ eingeflossen. (Mayerhofer, 2014, S.3-9)
} 
F 43.1: Posttraumatische Belastungsstörung (vgl. ICD-10 BMGF 2017, S.221) und F62.0: Andauernde Persönlichkeitsänderung nach Extrembelastung definiert (vgl. ebd., S.233). Zum besseren Verständnis werden die beiden genannten Definitionen hier angeführt:

\section{F43.1 Posttraumatische Belastungsstörung}

Diese entsteht als eine verzögerte oder protrahierte Reaktion auf ein belastendes Ereignis oder eine Situation kürzerer oder längerer Dauer, mit außergewöhnlicher Bedrohung oder katastrophenartigem Ausmaß, die bei fast jedem eine tiefe Verzweiflung hervorrufen würde. Prädisponierende Faktoren wie bestimmte, z.B. zwanghafte oder asthenische Persönlichkeitszüge oder neurotische Krankheiten in der Vorgeschichte können die Schwelle für die Entwicklung dieses Syndroms senken und seinen Verlauf erschweren, aber die letztgenannten Faktoren sind weder notwendig noch ausreichend, um das Auftreten der Störung zu erklären. Typische Merkmale sind das wiederholte Erleben des Traumas in sich aufdrängenden Erinnerungen (Nachhallerinnerungen, Flashbacks), Träumen oder Albträumen, die vor dem Hintergrund eines andauernden Gefühls von Betäubtsein und emotionaler Stumpfheit auftreten. Ferner finden sich Gleichgültigkeit gegenüber anderen Menschen, Teilnahmslosigkeit der Umgebung gegenüber, Freudlosigkeit sowie Vermeidung von Aktivitäten und Situationen, die Erinnerungen an das Trauma wachrufen könnten. Meist tritt ein Zustand von vegetativer Übererregtheit mit Vigilanzsteigerung, einer übermäßigen Schreckhaftigkeit und Schlafstörung auf. Angst und Depression sind häufig mit den genannten Symptomen und Merkmalen assoziiert und Suizidgedanken sind nicht selten. Der Beginn folgt dem Trauma mit einer 
Latenz, die wenige Wochen bis Monate dauern kann. Der Verlauf ist wechselhaft, in der Mehrzahl der Fälle kann jedoch eine Heilung erwartet werden. In wenigen Fällen nimmt die Störung über viele Jahre einen chronischen Verlauf und geht dann in eine andauernde Persönlichkeitsänderung (F62.0) über. (ICD-10 BMGF 2017, S.221)

\section{F 62.0 Andauernde Persönlichkeitsänderung nach Extrembelastung}

Eine andauernde, wenigstens über zwei Jahre bestehende Persönlichkeitsänderung kann einer Belastung katastrophalen Ausmaßes folgen. Die Belastung muss extrem sein, dass die Vulnerabilität der betreffenden Person als Erklärung für die tief greifende Auswirkung auf die Persönlichkeit nicht in Erwägung gezogen werden muss. Die Störung ist durch eine feindliche oder misstrauische Haltung gegenüber der Welt, durch sozialen Rückzug, Gefühle der Leere oder Hoffnungslosigkeit, ein chronisches Gefühl der Anspannung wie bei ständigem Bedrohtsein und Entfremdungsgefühl, gekennzeichnet. Eine posttraumatische Belastungsstörung (F43.1) kann dieser Form der Persönlichkeitsänderung vorausgegangen sein.

Persönlichkeitsänderungen nach:

- andauerndem Ausgesetztsein lebensbedrohlicher Situationen, etwa als Opfer von Terrorismus

- andauernder Gefangenschaft mit unmittelbarer Todesgefahr

- Folter

- Katastrophen

- Konzentrationslagererfahrungen (ICD-10 BMGF 2017, S.233) 
Die beiden Definitionen der Symptome der posttraumatischen Belastungsstörung und der andauernden Persönlichkeitsänderung nach Extrembelastung verdeutlichen das Ausmaß und die Komplexität der Belastungen für die Überlebenden des Holocaust.

\subsection{Die Überlebensschuld}

Mehrere Psychoanalytiker wie William Niederland beschäftigen sich ab den 1960er Jahren in Zusammenhang mit der Erforschung der traumatischen Störungen der Überlebenden auch mit dem Begriff der sogenannten Überlebensschuld (vgl. Leys, 2011, S.87). Der Begriff der Überlebensschuld beschreibt die Qualen der Opfer, „[...] weil sie sich immer wieder die Frage stellen, warum sie überlebten und ihre Liebsten nicht." (Kaminer-Zamberk, 2013, S.78) Ruth Leys zitiert Niederland in ihrem Beitrag zum Tel Aviver Jahrbuch für deutsche Geschichte 2011 diesbezüglich wie folgt:

Es gehört in der Tat zu den bittersten Ironien dieser so tragischen und von Bitternis erfüllten Situation, [...], dass ausgerechnet die Opfer der Verfolgung von Scham begleitete Schuldgefühle, Neigung zu Selbstverurteilung und Selbstvorwürfen erleben müssen, was offensichtlich bei den Tätern viel weniger (wenn überhaupt) der Fall ist [...] Aufgrund meiner Arbeit mit Hunderten von Verfolgungsopfern weiß ich, dass ihre Schuld- und Schamgefühle von sehr tiefer Natur sind und manchen ihrer klinischen Manifestationen zugrunde liegen. (Niederland, o.J., zit.n. Leys, 2011, S.96)

Überlebensschuld wird mit der Identifizierung der Überlebenden mit den verstorbenen Opfern in Zusammenhang gebracht. Niederland schreibt dazu:

Gestützt auf meine langjährigen Untersuchungen, habe ich Grund zur Annahme, dass das Überleben unbewusst als Verrat an den toten Eltern und Geschwistern empfunden wurde und dass das Weiterleben an sich 
mit einem anhaltenden Konflikt verbunden ist und eine Quelle ständiger Schuld- und Angstgefühle darstellt. (Niederland, 1981, S.421 zit.n. Leys, 2011, S.112)

Im Jahr 1961 beschrieb Niederland die emotionalen Störungen bei HolocaustÜberlebenden in einem Artikel im ,Journal of the Hillsdale Hospital'. Dies setze eine über ein Jahrzehnt andauernde Debatte über die Bedeutung von psychiatrischen Folgestörungen, ausgelöst durch die nationalsozialistische Verfolgung, in Gang (vgl. Leys, 2011, S.92). Die Erforschung der Überlebensschuld und deren psychischen Auswirkungen wurde vor allem von Psychoanalytikern in der Nachkriegszeit in den Vereinigten Staaten betrieben (vgl. ebd. S.91).

Einer der Auslöser für die Forschungen waren die Folgen der ersten deutschen Entschädigungsgesetze, die es den Überlebenden ab 1953 ermöglichten, „[...] Entschädigung für verfolgungsbedingten Gesundheitsschaden zu beantragen." (ebd.) Von vielen Ärzten wurden psychische Folgen der Verfolgung hingegen nicht anerkannt, sondern als sogenannte ,Rentenneurose' bezeichnet, „[...] als Neurose, die schlicht auf den Wunsch des Patienten, sich eine Rente zu sichern, zurückzuführen sei." (ebd. S.92) Man stützte sich damit auf eine psychoanalytische Theorie, nach derer die menschliche Persönlichkeit bereits mit 6 Jahren voll entwickelt sei und daher „[...] könnten die Probleme der Überlebenden nicht den Erlebnissen im Konzentrationslager angelastet werden." (ebd.)

Niederland protestierte gegen diese These und versuchte die Zusammenhänge zwischen den Erlebnissen im Holocaust und den zahlreichen psychiatrischen Störungen bei den Überlebenden aufzuzeigen (vgl. ebd., S.93). Er war als Gutachter der Bundesrepublik Deutschland in New York tätig, wo er für die Antragstellung im Rahmen des Bundesentschädigungsgesetzes, welches 1956 - rückwirkend 1953 verabschiedet wurde, Hunderte von Holocaust-Überlebenden untersuchte (vgl. Grandsard, 2011, S.212). Mit seiner Arbeit wies Niederland damit den Zusammenhang zwischen der Verfolgung durch das nationalsozialistische Regime und den daraus resultierenden traumatischen Störungen nach (vgl. ebd.). 
Er fasste die zahlreichen Symptome unter dem Begriff Holocaust-Syndrom zusammen und erklärte den Zusammenhang der Überlebensschuld mit zeitverzögert auftretenden Symptomen. Diese Verzögerung trat durch verschiedene Vermeidungsstrategien der Schuldgefühle der Überlebenden ein. Sobald die Schuldgefühle jedoch wieder ins Bewusstsein traten, konnten sich die Symptome manifestieren (vgl. Leys, 2011, S.96).

Der Psychiater Robert Jay Lifton sieht in der Überlebensschuld ebenfalls die Identifizierung mit den toten Opfern, „[...] aber vor allem als Verantwortungsgefühl gegenüber den Toten, das manche Überlebenden dazu veranlassen soll, es als permanente Aufgabe zu betrachten, Zeugnis abzulegen." (ebd., S.113)

\subsection{Die transgenerationale Weitergabe von Traumata}

Die posttraumatische Belastungsstörung, die erst spät vielen Überlebenden des Holocaust attestiert wurde, hatte zur Folge, dass es vielfach zu einer sogenannten transgenerationalen Weitergabe der Traumata an deren Nachkommen kam. Die ungelösten Traumata der Eltern führten oft zu Folgestörungen der Kinder (vgl. Brisch, 2013, S.43). Nach Natascha Unfried wird unter „[...] >>transgenerationaler Weitergabe $<<$ verstanden, dass die Elterngenerationen an die Generation der Kinder und Enkel ihre Vorstellungen, Verhaltensweisen, Scham- und Schuldgefühle, aber auch ihre Geheimnisse und unverarbeiteten Traumata weitergibt." (Unfried, 2013, S.50) Die Folgen dieser ungelösten Traumata können sich nicht nur auf die zweite, sondern auch auf die dritte und vierte Nachfolgegeneration auswirken und zu Folgestörungen führen (vgl. ebd.).

Marianne Rauwald und Rosalba Maccarrone Erhardt beschreiben die Folgen von traumatischen Erlebnissen für die Nachfolgegeneration wie folgt:

Extreme Traumata hinterlassen häufig schwerwiegende chronische Traumafolgestörungen, die sich in unterschiedlichen Beschwerden manifestieren, beinahe durchgängig jedoch gravierende Bindungs- und Beziehungsstörungen beinhalten. Besonders betroffen davon ist die 
Beziehung zu den eigenen Kindern. (Rauwald, Maccarrone Erhardt, 2013, S.57)

Der klinische Psychologe Natan P.F. Kellermann beschreibt vier Theorien zur Weitergabe dieser Traumata. Die erste Theorie geht aus psychoanalytischer Sicht davon aus, dass sich nicht verarbeitete Traumata der Überlebenden unbewusst auf die nachfolgenden Generationen übertragen können (vgl. Kellermann, 2011, S.145f). Die Familiensystemtheorie wiederum sieht die Weitergabe der Traumata in der fehlenden Kommunikation über das Erlebte (vgl. ebd.). Als dritte Theorie wird die Sozialisationstheorie erwähnt, welche die Weitergabe im Erziehungsstil verortet und die vierte, biologische Theorie genannt, sieht die Weitergabe traumatischer Erfahrungen genetisch bedingt (vgl. ebd. S.154). „Die Kinder schwer traumatisierter Holocaust-Überlebenden wären dann für die Posttraumatische Belastungsstörung (PTBS) „prädisponiert““” (Kellermann, 2011, S.154) In empirischen Untersuchungen konnte zwar nicht belegt werden, dass emotionale Störungen in der Nachfolgegeneration der Holocaust-Überlebenden allgemein häufiger vorkommen als bei anderen Personen der gleichen Altersgruppe, aber dass dennoch viele der Nachfolgegeneration unter den traumatischen Erfahrungen ihrer Eltern leiden (vgl. Kellermann, 2011, S.156). Viele zeigen eine hohe Anfälligkeit für posttraumatische Belastungsstörungen, andere jedoch entwickeln ein bemerkenswertes ResilienzVerhalten, welches oft mit den Überlebens- und Bewältigungsstrategien der Eltern korreliert (vgl. ebd. S.159).

Diese Bewältigungsstrategien der Überlebenden zeigten sich oft in einem ausgeprägten Vermeidungsverhalten, wie es auch in den Symptomen der posttraumatischen Belastungsstörung beschrieben ist. Dieses Vermeidungsverhalten resultierte auch im Schweigen der Opfer über das Erlebte. Das Schweigen der Eltern hatte Auswirkungen auf die Kinder, wie Elli I. Kaminer-Zamberk verdeutlicht.

Es waren aber auch die Kinder, die sich nicht trauten zu fragen. Sie spürten die tiefe Trauer der Eltern und hatten Angst schmerzliche Wunden aufzureißen. So schwiegen die Eltern, um die Kinder zu schützen, und die 
Kinder stellten keine Fragen, um die Eltern zu schonen. (KaminerZamberk, 2013, S.87)

Die Traumata, die in den meisten Fällen nicht verarbeitet wurden beziehungsweise verarbeitet werden konnten, übertrugen sich so, unbewusst allein nur durch das Zusammenleben mit den traumatisierten Eltern, auf die nachfolgenden Generationen (vgl. Sänger, Udolf, 2013, S.139). „Diese Kinder- und mittlerweile Enkelgenerationen zeigten häufig ähnliche oder genau die gleichen, für Traumatisierungen typische Folgeerscheinungen wie die Eltern bzw. Großeltern, die der Nazi-Herrschaft und dem Holocaust ausgeliefert waren - obwohl sie selbst solchen Erlebnissen nie ausgesetzt waren." (ebd.)

Die Nachfolgegenerationen nahmen ab den 1960er Jahren, im Gegensatz zu der Generation der Überlebenden, häufiger therapeutische Hilfe in Anspruch (vgl. Kaminer-Zamberk, 2013, S.80). Die Erforschung der psychischen Probleme und Traumata der Überlebenden und der nachfolgenden Generation begann ebenfalls in dieser Zeit. Es folgten Studien, durch die die Traumatisierungen und Folgestörungen klassifiziert und in weiterer Folge zu behandeln versucht wurden (vgl. ebd., S.78).

Im hohen Alter kann es zu einer Retraumatisierung beziehungsweise einer Reaktivierung der Traumata kommen und sowohl bei der posttraumatischen Belastungsstörung als auch bei der transgenerationalen Weitergabe von Traumata auftreten. Eigene und/oder transgenerational weitergegebene Traumata können im Alter nicht mehr so erfolgreich kompensiert beziehungsweise abgewehrt werden. Verstärkte Angstgefühle, bedingt durch Hilflosigkeit aufgrund eventuell auftretender Einbußen der motorischen Fähigkeiten oder des Gedächtnisses begünstigen diese Retraumatisierung (vgl. Schrader, 2013, S.129). 


\section{5. Österreich und die Opferthese}

Die ersten Jahrzehnte nach dem Ende des Zweiten Weltkriegs waren von Schweigen und Verdrängung geprägt. Das offizielle Österreich hat sich, auch aufgrund der Moskauer Deklaration von 1943, nach dem Ende der nationalsozialistischen Herrschaft als deren Opfer dargestellt (vgl. Perz, 2002, S.150). Die Moskauer Deklaration wurde am 30. Oktober 1943 von den Außenministern von England, den USA und der Sowjetunion beschlossen und bezeichnete Österreich als „[...] das erste freie Land, das der Hitlerschen Aggression zum Opfer gefallen ist [...].“3

Die Darstellung Österreichs als erstes Opfer wird allerdings nur im ersten Absatz der Moskauer Deklaration beschrieben. Im letzten Absatz wurde Österreich bereits eine Mitverantwortung attestiert, die wie folgt lautet:

Österreich wird jedoch darauf aufmerksam gemacht, dass es für die Beteiligung am Kriege auf seiten [sic] Hitlerdeutschlands die Verantwortung trägt, der es nicht entgehen kann, und dass bei der endgültigen Regelung unvermeidlich sein eigener Beitrag zu seiner Befreiung berücksichtigt werden wird. ${ }^{4}$

Dieser Absatz wurde jedoch ignoriert, da er sich nicht in das Bild der Opferthese fügte. Albert Sternfeld beschreibt in seinem Buch ,Betrifft: Österreich` im Jahr 1990 die österreichische Auslegung der Moskauer Deklaration (vgl. Sternfeld, 1990, S.39). Er berichtet darin über die Publikation des ,Rot - Weiß - Rot - Buches', welches 1946 von der österreichischen Bundesregierung nach amtlichen Quellen herausgegeben wurde, wie auch Heidemarie Uhl in einem Referat erläutert. ${ }^{5}$ Das Buch sollte den österreichischen Widerstand und den eigenen Beitrag Österreichs zu seiner Befreiung verdeutlichen und somit die Auflagen der Alliierten, wie in der Moskauer Deklaration erwähnt, erfüllen. Diese Argumentation führte zu einer

\footnotetext{
${ }^{3}$ https://www.hdgoe.at/moskauer-deklaration [Datum des Zugriffs: 30.8.2019]

${ }^{4} \mathrm{ebd}$.

${ }^{5}$ http://www.klahrgesellschaft.at/Referate/Uhl_2003.html [Datum des Zugriffs: 30.8.2019]
} 
Externalisierung. ${ }^{6}$ Man konnte sich dadurch als Opfer Hitlerdeutschlands darstellen, eine Mitverantwortung abgeben und so die Opferthese erfüllen. Sternfeld sieht darin Vorbereitungen zur Formulierung des Staatsvertrages: „Im Hinblick auf die anlaufenden Bestrebungen, den Staatsvertrag zu erlangen, war man bemüht, Österreich in ein möglichst günstiges Licht zu stellen." (Sternfeld, 1990, S.39) In der österreichischen Regierungsproklamation von 1947 wurde die Opferthese dann vom Land auf das gesamte österreichische Volk erweitert und im österreichischen Staatsvertrag von 1955 wurde erreicht, „[...] einen Passus über die Mitverantwortung Österreichs am Krieg in letzter Minute noch zu streichen." (Perz, 2002, S.151)

Doch nicht nur die Haltung des offiziellen Österreich in der ersten Nachkriegszeit, sondern auch das Schweigen der Überlebenden des Holocaust trug zur Etablierung der Opferthese bei. Erinnern und Gedenken fanden einseitig wenn, dann in Form von Denkmälern für die gefallenen Freiheitskämpfer und Wehrmachtssoldaten statt, die die Opferthese noch mehr betonten (vgl. ebd., S.154).

Ab Mai 1945 gab es Bemühungen einer Entnazifizierung. Volksgerichte, die eigens dafür geschaffen wurden, nahmen sich dieser an. Es folgten das „[...] Verbotsgesetz für alle NS-Organisationen [...], gefolgt von einem Gesetz über die Behandlung von Kriegsverbrechern." (ebd., S.152) Diese Bemühungen fanden jedoch ein rasches Ende. Bereits 1947 wurde ein Nationalsozialistengesetz beschlossen, das zwischen „[...] minderbelasteten und belasteten Nationalsozialisten [...]" (ebd. S.153) unterschied und zu einer Amnesie und Reintegration von 90 Prozent der ehemaligen Nationalsozialisten führte (vgl. ebd.). Auch die Strafverfolgung durch die Justiz ging zurück. In den wenigen Verfahren, die stattfanden, konnten die Geschworenen zu keinen Urteilen kommen. „Die Verfolgung nationalsozialistischer Gewaltverbrechen in Österreich wurde somit eingestellt und nicht wieder aufgenommen." (ebd. S.154)

Die Haltung des offiziellen Österreich spiegelte sich auch in der Restitutionsfrage von geraubtem Eigentum wider. Der damalige Innenminister Oscar Helmer sprach sich in der 132. Ministerratssitzung am 9. November 1948 für eine Verzögerungstaktik aus, indem er den folgenden Ausspruch tätigte: „Ich bin dafür, die Sache in die Länge zu ziehen." (Knight, 2000, S.9)

\footnotetext{
${ }^{6}$ http://www.klahrgesellschaft.at/Referate/Uhl_2003.html [Datum des Zugriffs: 30.8.2019]
} 
Zwischen 1946 und 1949 wurden insgesamt sieben Rückstellungsgesetze beschlossen. ${ }^{7}$ Diese Gesetze betrafen ausschließlich Naturalrestitution. Es war für die Betroffenen sehr schwierig sich zu orientieren, welches Gesetz auf ihre jeweiligen Entschädigungsansprüche anzuwenden war und die Entschädigungen erfolgten nur beschränkt. $^{8}$

Sternfeld, der selbst in einem Flüchtlingstransport für Kinder Österreich im Jahr 1938 verlassen musste, erläutert in seinem Buch den Umgang Österreichs mit der Nachkriegsgeschichte und die Problematiken der Entschädigungsforderungen. Diesbezüglich geht er auch auf die Thematik der Rückkehr der Vertriebenen ein. Als sogenannte ,Displaced Persons' wurden Zwangsarbeiter, Kriegsgefangene und KZHäftlinge bezeichnet, die sich nach dem Ende des Zweiten Weltkrieges außerhalb des Heimatlandes befanden. Der Begriff wurde von den Alliierten geprägt. ${ }^{9}$ Laut Sternfeld hätte sich die Regierung mit den Displaced Persons und deren Rückkehr nach Österreich auseinandersetzen müssen. Er zitiert einen Bericht des Innenministeriums vom 14. Oktober 1947, in dem festgehalten wurde, dass sich 593.774 Displaced Persons und Flüchtlinge auf österreichischem Staatsgebiet befanden (vgl. Sternfeld, 1990, S.71). Die Haltung der Gesellschaft und auch der Politik gegenüber den Zurückgekehrten war jedoch allgemein ablehnend, nicht zuletzt auch aufgrund der wirtschaftlichen Situation, des Mangels an Wohnungen und Nahrungsmitteln. Diese allgemeine Haltung beschreibt er wie folgt: „Volk und Regierung gingen vom Motto aus ,Wir haben nichts, wir sind selbst arm, wir sind zerbombt, Wohnungsnot usw.', und daran schloss sich folgerichtig die Einstellung ,Wir können doch nicht noch weitere Mäuler füttern'." (ebd., S.71)

Sternfeld schreibt:

Aus den Wortprotokollen der Regierungssitzungen 1945-1947 geht schlüssig hervor, daß ein Aufruf zur Rückkehr nicht beabsichtigt war; daß man der Rückkehr „allzu Vieler“ negativ gegenüberstand; daß man den

\footnotetext{
${ }^{7} \mathrm{https}: / /$ www.nationalfonds.org/weitere-rechtsquellen-und-parlamentarische-materialien [Datum des Zugriffs:1.9.2019]

${ }^{8} \mathrm{ebd}$.

${ }^{9}$ https://www.hdgoe.at/displaced-persons [Datum des Zugriffs: 30.8.2019]
} 
trotz alledem Zurückgekehrten nicht immer bei ihrer zwangsläufig notwendigen Wiedereingliederung hilfreich zur Seite stand. (Sternfeld, 1990, S.77)

Diese Haltung zeigte sich im allgemeinen Sprachgebrauch. Die Vertriebenen wurden allgemein als EmigrantInnen bezeichnet. Sternfeld veröffentlicht in seinem Buch ein Zitat von Leopold Figl vom 22. November 1945 aus der Zeitung ,Kleines Volksblatt': „Es war für die Emigranten sicherlich bequemer, in ihren Clubsesseln zu sitzen, als für Österreich zu leiden." (Sternfeld, 1990, S.19) Hier verdeutlicht sich die Verharmlosung der Geschichte der zur Flucht gezwungenen Personen. Wie Sternfeld formuliert, war dieser Kreis an Personen „[...] zur Auswanderung gezwungen, gehetzt und verließ auf jedem nur möglichen Weg, unter Zurücklassung allen Besitzes, das Land. Es waren ,Vertriebene“ und nicht ,Emigranten'." (ebd. S.20)

Ebenso verhielt es sich mit der Einstellung der ÖsterreicherInnen zu Restitution und Wiedergutmachung. Wenn die EmigrantInnen es im Ausland besser gehabt hätten als in Österreich, warum sollte man dann etwas wieder gut machen? Sternfeld drückt seinen Unmut gegenüber der Wortwahl ,Wiedergutmachung' generell aus. Für ihn birgt der Begriff ein Missverständnis, denn Eigentum, das geraubt wurde, wieder zurückzugeben, stellt für inn eine Selbstverständlichkeit in einem Rechtsstaat dar (vgl. ebd., S.18).

Sternfeld geht in seinem Buch auch auf die Problematik der Entschädigungen ein. Die materiellen Verluste durch die Enteignungen und Arisierungen waren feststellbar. Wie verhielt es sich jedoch mit den immateriellen Schäden, mit den physischen und psychischen Folgeschäden, zum Beispiel von Konzentrationslagererfahrungen? Sternfeld sprach sich auch für eine Entschädigung aufgrund des erzwungenen Ausbildungsabbruchs bei Schülerlnnen und StudentInnen aus (vgl. ebd., S.18f). Man (die österreichischen Bundesregierungen nach dem Jahr 1945, Anm. d. Verf.) berief sich jedoch auf die Opfertheorie und

[...] war aber eindeutig nicht bereit, von sich aus auf lange Sicht gesehen den Themenkomplex in einer Weise zu behandeln, der den Geschädigten 
und Vertriebenen einigermaßen befriedigend erscheinen würde, und dies in Kenntnis der negativen Auswirkungen, die diese Art der Behandlung in aller Welt hervorrufen würde. (Sternfeld, 1990, S.86)

Es sollte bis in die 1980er Jahre dauern, bis Österreich sich der eigenen Vergangenheit und der Mitverantwortung am Nationalsozialismus stellen sollte. Der Auslöser war die sogenannte Waldheim-Affäre, auf die im Kapitel 2.8.2. näher eingegangen wird. Anlässlich des bevorstehenden Gedenkjahres 1988 wurde unter anderem ein Beitrag von Hugo Portisch in der Zeitung ,Kurier am 21. Jänner 1987 veröffentlicht, in dem er die Opferthese kritisierte. „[...] das war ein unwahres und leider auch schäbiges und würdeloses Verhalten, das Jahrzehnte an unserem Ruf gezerrt hat, und die Rechnung wird uns jetzt präsentiert [...]“ (Portisch, 1987, zit.n. Sternfeld, 1990, S.157) Portisch sprach sich in dem Beitrag für eine Verantwortungsübernahme und Entschädigung aus (vgl. Sternfeld, 1990, S.157).

Im Gedenkjahr 1988 wurden zahlreiche Publikationen veröffentlicht. Es gab viele Veranstaltungen, allerdings wurde kein Sonderbeauftragter ernannt, der die Veranstaltungen koordinieren sollte, wie von Sternfeld vorgeschlagen, somit fanden viele zeitgleich und mit teilweise denselben Vortragenden und dem gleichen Publikum statt (vgl. ebd., S.163). Sternfeld hatte etliche dieser Veranstaltungen besucht, auch bereits vor und nach dem Gedenkjahr 1988 und er bemerkte, dass „[...] sie von einem relativ kleinen Kreis, etwa 20-25 Historikern, Politologen, Sozialwissenschaftlern, bestritten werden und dass das interessierte Kernpublikum aus 50-80 treuen, regelmäßigen Zuhörern oder Teilnehmern besteht." (ebd. S.163) Das Gedenkjahr 1988 sollte ein breiteres Publikum ansprechen, was laut Sternfeld nicht in dem Maße wie erhofft, passiert ist (vgl. ebd., S.164). Er hält jedoch Folgendes fest:

Meiner Meinung nach hat ein Nachdenk- und Umdenkprozeß bei den jüngeren Generationen eingesetzt, soweit es sich nicht um „unbelehrbare Kreise" handelt. Bei jenem Teil der Kriegsgeneration, der mit der dem 
Dritten Reich gegenüber erfüllten Pflicht gut lebt, hat das Gedenkjahr nichts bewirkt. (Sternfeld, 1990, S.166)

Die Bundesregierung gab keine Stellungnahme zu einer Verantwortungsübernahme im Rahmen des Festaktes in der Hofburg im Gedenkjahr 1988 ab, wie von Sternfeld, Portisch und anderen bemerkt: „Die Führungsspitze konnte sich zu einer derartigen Erklärung nicht durchringen. Die große Rede wurde nicht gehalten, eine große Chance war vertan." (ebd.)

Ein offizielles Schuldbekenntnis erfolgte erst durch die vielbeachtete Rede von Bundeskanzler Dr. Franz Vranitzky vor dem Nationalrat am 8. Juli 1991. Darin hieß es unter anderem: „Viele Österreicher waren an den Unterdrückungsmaßnahmen und Verfolgungen des Dritten Reichs beteiligt, zum Teil an prominenter Stelle. Über eine moralische Mitverantwortung für Taten unserer Bürger können wir uns auch heute nicht hinwegsetzen." (Stenographisches Protokoll der 35. Nationalratssitzung, 1991, S.15) 1993 folgte dann eine offizielle Entschuldigung für die Täterschaft von Österreichern anlässlich einer Israel-Reise von Bundeskanzler Vranitzky. Damit bekannte sich das offizielle Österreich zu seiner Mitverantwortung (vgl. Stuhlpfarrer, 2002, S.238). 1997 erfolgte der Beschluss auf Bundesebene, den 5. Mai - den Tag der Befreiung des Konzentrationslagers Mauthausen - fortan als jährlichen Gedenktag gegen Gewalt und Rassismus zu konstituieren (vgl. ebd.).

\subsection{Von der Vergangenheitsbewältigung zur Erinnerungskultur}

Wie eingangs dargelegt, war die Nachkriegszeit in Österreich von Schweigen und Verdrängung hinsichtlich der Erinnerung an das NS-Regime geprägt. Bertrand Perz schreibt dazu: „In einem Staat, der sich und seine Gesellschaft als Opfer des Nationalsozialismus sah, war für die Erinnerung an die NS-Gewaltverbrechen und die Auseinandersetzung mit der eigenen Verantwortung für diese Verbrechen wenig Platz." (Perz, 2002, S.160)

Der Begriff der Vergangenheitsbewältigung stand in engem Zusammenhang mit dem Wunsch, mit der Geschichte abzuschließen. Sternfeld übt Kritik an diesem Begriff, Vergangenheitsbewältigung beinhalte „[...] eine Endgültigkeit, die man in der 
Auseinandersetzung mit seiner eigenen Geschichte wohl kaum erreichen kann.“ (Sternfeld, 1990, S.127) Assmann sieht in der Vergangenheitsbewältigung das Ziel, „[...] die Überwindung einer schmerzhaften Erinnerung um einer gemeinsamen und freien Zukunft willen“ (Assmann, 2018, S.71) zu erreichen.

Assmann beschreibt in ihren Büchern die erste Wende im Umgang mit der Erinnerung. Das Schweigen fand sein Ende in den 1960er Jahren mit der sogenannten ,68er-Generation‘, die Nachfolgegeneration der Kriegsgeneration. Diese Generation begann zu hinterfragen und auch anzuklagen (vgl. Assmann, 2016a, S.46ff). Es kam zu einem intergenerationellen Konflikt, in welchem „[...] nicht die Kommunikation befördert, sondern das Schweigen vertieft wurde. Während die Integration der Gesellschaft fortschritt, brachen die Familien auseinander. An die Stelle von Fragen traten lautstarke Anklagen, Gespräche gingen in Vorwürfen und Rechtfertigungen unter." (ebd., S.47) Assmann beschreibt damit einen Kampf der Generationen.

So wie die Überlebenden des Holocaust über ihr Leid schwiegen, so schwieg auch die Gesellschaft in Deutschland und Österreich. Der Philosoph Hermann Lübbe bezeichnete dies im Jahr 1983 als ,absichtsvolles Schweigen‘ (vgl. ebd., S.43).

Natürlich konnte ein ganzes Volk nicht schlagartig vergessen, aber es konnte sich darauf einigen, über die schlimme Vergangenheit, die man soeben hinter sich hatte, zu schweigen. Vor allem wollte man nicht mehr Auskunft geben über die eigene Begeisterung und Zustimmung, über alle Aktivitäten, Hoffnungen und Emotionen [...]. (ebd.)

Die Bewältigung der Vergangenheit bestand somit auf gesellschaftlicher Ebene, auf Täter- und auf Opferseite aus Schweigen. Dan Bar On, ein israelischer Psychologe und Therapeut, betitelt das Schweigen auf beiden Seiten als die ,doppelte Mauer des Schweigens'. Als die Überlebenden ihr Schweigen und damit die erste Mauer brechen wollten, fanden sie sich vielfach von einer zweiten Mauer des Schweigens umgeben, dem Schweigen der Gesellschaft (vgl. Assmann, 2018, S.99). Die 68er- 
Generation stellte sich diesem Schweigen schließlich vehement entgegen und begann die Vergangenheit aufzuarbeiten. Sie stellte sich damit vielfach gegen die eigene Elterngeneration (vgl. Assmann, 2016a, S.53).

Auch Margrit Frölich, Ulrike Jureit und Christian Schneider nehmen Bezug auf den Generationenwechsel. Ihnen zufolge unterscheiden sich die Wahrnehmungen der an historischen Ereignissen Beteiligten von denen der Nachgeborenen und beschreiben damit den Konflikt, der zwischen den Generationen ausgetragen wurde (vgl. Frölich, Jureit, Schneider, 2012, S.15).

Der sogenannte ,memory boom' (Erinnerungsboom), wie es Andreas Huyssen formuliert, begann in den 80er Jahren des 20. Jahrhunderts (vgl. Radonić, Uhl 2016, S.8). Gemeint ist damit der Paradigmenwechsel in der Auseinandersetzung mit der Erinnerung an den Holocaust und das Entstehen von Erinnerungskulturen, wie wir sie heute verstehen. Der Begriff Erinnerungskultur selbst wurde dann schließlich erst in den 1990er Jahren geprägt (vgl. Assmann, 2016a, S.54). Er setzte sich „[...] in wissenschaftlichen Diskursen, in den Ansprachen der Politiker, aber auch in den Medien und in der Altagssprache immer mehr [...]" (Assmann, 2016b, S.29) durch.

Laut Frölich, Jureit und Schneider beziehen sich Erinnerungskulturen „[...] auf starke historische Ereignisse beziehungsweise erklären sie zu solchen. Sie greifen Handlungszusammenhänge auf, die kollektive Prägekraft haben - positiv oder negativ." (Frölich, Jureit, Schneider, 2012, S.15) Sie führen weiter aus, dass sich der Begriff Erinnerungskultur mittlerweile nahezu ausschließlich auf die kollektive Erinnerung an den Holocaust bezieht (vgl. ebd.).

Im Jahr 2002 weisen Volkhard Knigge und Norbert Frei darauf hin, dass der Erinnerungsboom in seiner Quantität nichts über die Qualität aussagen würde. „Zugleich täuscht der Erinnerungsboom des vergangenen Jahrzehnts leicht darüber hinweg, daß eine nicht nur moralische, sondern auch kontinuierliche, praxisinformierte, curriculare, geschichtsdidaktisch-pädagogische, medientheoretische, museologische und ästhetische Debatte hinsichtlich der Gestaltung dieser Erinnerung weitgehend fehlt." (Knigge, Frei, 2002, o.S.) 
Einerseits trugen die Forschungen zum Trauma der Überlebenden seit den 1960ern zur Auseinandersetzung mit der Vergangenheit bei. Andererseits kann man, rückblickend gesehen, einige Meilensteine, die zur Etablierung der deutschen (und österreichischen) Erinnerungskultur führten, feststellen. Diese werden später noch erläutert.

Anton Pelinka begründet in seinem Vorwort zur zweiten erweiterten Auflage des Buches von Sternfeld im Jahr 2001 die verzögerte Auseinandersetzung mit dem Holocaust wie folgt:

Es ist, als ob alle - die Täter, aber auch die Opfer, und erst recht diejenigen, die weder Täter noch Opfer waren - die Dimension der Shoa zunächst zu begreifen nicht in der Lage waren; als ob es erst den Abstand einer neuen Generation gebraucht hätte, um die Welt zu einer entsprechenden Reaktion zu bringen. (Pelinka, 2001, S.16)

\subsection{Opferidentifizierung und Opferorientierung}

Diese neue Erinnerungskultur stellte emotionale, moralische und kulturelle Ansprüche, wie es Assmann formuliert (vgl. Assmann, 2016a, S.58). Jureit und Schneider sprechen von einer Erinnerungskultur, die sich mit den Opfern identifiziert. Heidemarie Uhl schreibt dazu: „Der Paradigmenwechsel vom heroischen zum viktimologischen Narrativ sollte sich in den 1980er Jahren durchsetzen." (Uhl, 2016, S.89) Grund dafür war laut Martin Sabrow der Generationenwechsel (vgl. Sabrow, 2012a, S.45). Nach Charles M. Maier konnte erst durch den Generationenwechsel eine „[...] selbstkritische Auseinandersetzung mit der schuldhaften Verstrickung der eigenen Gesellschaft in ein Terror- und Gewaltregime [...]" (Uhl, 2016, S.104) stattfinden.

Jureit und Schneider prägten den Begriff der ,gefühlten Opfer'. Gemeint war damit, dass sich die erste deutsche Nachfolgegeneration von ihrer Elterngeneration lossagen und sich dafür mit den Opfern identifizieren würde. Sie bezeichnen diese Lossagung als Externalisierung. Später folgte dann mit einer Internalisierung „, $[\ldots]$ 
das erst später einsetzende Verhältnis zu den konkreten jüdischen Opfern, das aus einem neuen Sinn der Verantwortung für die ererbte Schuld entstanden ist." (Assmann, 2016a, S.53) Sie orteten jedoch eine Art Selbstbetrug der 68erGeneration, um sich von der eigenen Geschichte zu befreien (vgl. ebd., S.61). Jureit formuliert dies wie folgt: „Wir tun schlicht so, als wenn es um Geschehnisse geht, die wir selbst erfahren oder erlitten haben, und simulieren einen Selbstbezug, in den wir uns dann emotional hineinsteigern." (Jureit, 2012, S.27) Die Identifizierung und Empathie mit den Opfern stellte laut Assmann jedoch keinen, wie von Jureit vermutet, „[...] Irrweg und Skandal der deutschen Erinnerungskultur, sondern deren Voraussetzung und Grundlage [...]" (ebd., S.63) dar. Die Begriffe der opferidentifizierten Erinnerung und der gefühlten Opfer waren jedoch missverständlich, wie Werner Konitzer erläutert.

Die Rede, dass sich eine Person mit einem Opfer oder mit den Opfern eines Verbrechens identifiziert, ist jedoch mehrdeutig. Sie kann einerseits bedeuten, dass sich diese Person mit dem Opfer des Verbrechens gleichsetzt, sich also selbst gleichsam an dessen Stelle begibt und dessen Rolle einnimmt. Sie kann aber auch so verstanden werden, dass sich die Person auf die Seite des Opfers stellt, dass sie sich also darum kümmert, dass demjenigen, der Opfer eines Verbrechens geworden ist, Gerechtigkeit widerfährt, dass sie damit die Angelegenheit aus Gründen der Gerechtigkeit zu ihrer Sache macht. (Konitzer, 2012, S.119)

Konitzer verwendet in Hinblick auf letztere Bedeutung der Identifizierung den Terminus des opferorientierten Gedenkens. Das opferorientierte Gedenken sei vom opferidentifizierten Gedenken zu trennen (vgl. ebd., S.120).

Sowohl Jureit und Schneider als auch Konitzer beschreiben damit die Probleme in der Auseinandersetzung mit der Vergangenheit. Schneider sieht in der Identifizierung den „[...] zentralen Wunsch, unschuldig zu sein und den Schrecken, der aus ihrer 
Genealogie nicht zu tilgen war, ungeschehen zu machen." (Schneider 2010, S.121, zit.n. Konitzer, 2012, S.126)

In Deutschland kann man von einer opferorientierten Erinnerungskultur sprechen. Opferidentifizierung ist laut Assmann aufgrund der Täterrolle Deutschlands de facto nicht möglich (vgl. Assmann, 2016a, S.65f). In diesem Zusammenhang wird von einem negativen Gedächtnis gesprochen. Assmann beschreibt den Begriff des negativen Gedächtnisses wie folgt: „Die Schuld, die den nachwachsenden Generationen nicht mehr angelastet werden kann, wird dabei in ,historische Verantwortung" verwandelt und als Warnung in die Zukunft verschoben." (ebd. S.66)

\subsection{Meilensteine der Erinnerungskultur}

Im folgenden Kapitel werden die wichtigsten Meilensteine in der Entstehung der Erinnerungskultur angeführt. Auch wenn in der Literatur häufig nur von der deutschen Erinnerungskultur geschrieben wird, so betreffen die Meilensteine, die in dieser Arbeit beschrieben werden, die internationale und auch die österreichische Erinnerungskultur.

\subsubsection{Die Fernsehserie „Holocaust - Die Geschichte der Familie Weiss“}

Der erste dieser Meilensteine war die Ausstrahlung der vierteiligen, amerikanischen Fernsehserie „Holocaust - Die Geschichte der Familie Weiss“, welche im Jänner $1979 \mathrm{im}$ deutschen Fernsehen gezeigt wurde. Erst durch diese Serie fand der Begriff ,Holocaust' Eingang in den deutschen Sprachgebrauch. Nach Daniel Levy und Nathan Sznaider wurde der Begriff amerikanisiert. „Mit dem Wort war auch ein Rahmen geschaffen. Man konnte über das Ereignis reden, darüber nachdenken, ohne es umschreiben zu müssen." (Levy, Sznaider, 2007, S.138f)

,Holocaust' stammt aus dem Griechischen und bedeutet „vollständig verbrannt“. Der Begriff bezog sich ursprünglich auf religiöse tierische Brandopfer. Durch die 
Ausstrahlung der Serie ,Holocaust' wurde es zum Synonym für die Judenvernichtung durch das nationalsozialistische Regime. ${ }^{10}$

Assmann beschreibt die Wirkung der Serie auf die ZuseherInnen:

Zum ersten Mal war die gesamte Gesellschaft von der Empathie mit den jüdischen Opfern ergriffen; die emotionalisierende Darstellung dieses Films vermochte es, anhand einzelner Personen und ihrer Biographien eine Brücke zu schlagen über den Abgrund der politisierten deutschen Vergangenheit. (Assmann, 2016a, S.55)

Die Serie handelt einerseits von der fiktiven Familie Weiss, eine deutsch-jüdische Familie, durch deren Verfolgungsschicksal das Leid von Millionen Verfolgten verdeutlicht wurde, und andererseits von der deutschen Familie Dorf, die als MitläuferInnen des Regimes dargestellt wird und deren Sohn Karriere bei der SS [Schutzstaffel, Nationalsozialistische Organisation, Anm. d. Verf] macht. ${ }^{11}$ Die Darstellung der Opfer aus einer emotionalen Perspektive heraus machte eine Identifizierung mit den fiktiven Charakteren der Serie möglich. Der zweite Handlungsstrang betraf die Geschichte der TäterInnen anhand der Figur des Erik Dorf. „Die Figur Dorf sollte zeigen: Jeder kann zum funktionierenden Rädchen der Verbrechensmaschinerie werden. “12

Anlässlich des 40jährigen Jubiläums wurde die Serie im Jänner 2019 erneut ausgestrahlt und die damalige Resonanz auf die Serie durch die Dokumentation ,Wie ,Holocaust' ins Fernsehen kam' von Alice Agneskirchner beleuchtet. ${ }^{13}$,Holocaust" löste eine Schockwelle aus, wie es die Süddeutsche Zeitung im Jänner 2019 berichtet. Die Ausstrahlung der Serie 1979 wurde sehr kontrovers betrachtet, es kam

\footnotetext{
${ }^{10} \mathrm{https}: / /$ www.dw.com/de/wie-der-begriff-holocaust-\%C3\%BCberhaupt-nach-deutschland-kam/a47254116 [Datum des Zugriffs: 30.8.2019]

${ }^{11} \mathrm{https}$ ://www.srf.ch/kultur/film-serien/tv-serie-holocaust-wie-eine-serie-das-geschichtsbild-derdeutschen-umkrempelte [Datum des Zugriffs: 30.8.2019]

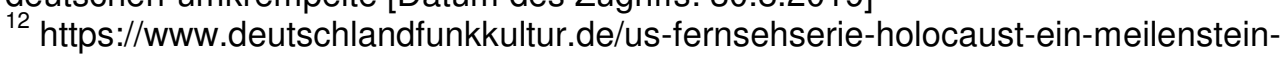

der.1005.de.html?dram:article_id=437527 [Datum des Zugriffs: 30.8.2019]

${ }^{13} \mathrm{https}$ ://www.faz.net/aktuell/feuilleton/medien/serien/ard-sender-wiederholen-die-amerikanischeserie-holocaust-15972921.html [Datum des Zugriffs: 30.8.2019]
} 
zu Morddrohungen, zu Sprengstoffanschlägen auf Sendemasten, die Fernsehsender wollten die Serie zuerst nicht ausstrahlen. Nach der Ausstrahlung gingen mehr als 23.000 Anrufe beim Sender ein, Kisten an Leserbriefen wurden geschickt, mehrheitlich positiv formuliert, doch es kam auch zu negativen Reaktionen, die zeigten, wie sehr Antisemitismus in der Gesellschaft noch verankert war. ${ }^{14}$

Der Grund für den Erfolg der Serie war der Perspektivenwechsel. Davor betraf das historische Interesse vor allem die TäterInnen, danach setzte man sich zum ersten Mal mit dem Leid der Opfer auseinander. Laut Uhl fand ein Paradigmenwechsel statt: „Die überwältigende Resonanz auf ,Holocaust' in der Bundesrepublik Deutschland und Österreich verweist auf neue Erinnerungsbedürfnisse, die von den heroisierenden Pathosformeln der Nachkriegsmythen offenkundig nicht mehr erreicht werden konnten." (Uhl, 2016, S.88) Etwa 15 Millionen Menschen sahen die Ausstrahlung der Serie, die Einschaltquote betrug 40 Prozent (vgl. Levy, Sznaider, 2007, S.139).

Assmann zufolge legte die Serie aufgrund der Empathie, die sie bei den ZuschauerInnen den Opfern des Holocaust gegenüber auslöste, den Grundstein für das spätere Verständnis von Erinnerungskultur (vgl. Assmann, 2016a, S.55). Die Reaktionen der ZuschauerInnen zeigten „[...] die Versäumnisse der deutschen Vergangenheitsbewältigung" (Levy, Sznaider, 2007, S.139) auf. Viele der Anrufe, die danach beim Sender WDR, der die Serie ausstrahlte, eingingen, bezogen sich auf die Frage, wie man den nachfolgenden Generationen erklären sollte, dass der Holocaust geschehen konnte und warum man nichts dagegen unternommen hatte. ${ }^{15}$

Die Ausstrahlung der Serie löste somit ungeahnte Debatten aus und zeigte das Ausmaß der Verdrängung der Vergangenheit auf. Die Serie beherrschte alltägliche Gespräche und forderte zu einer neuen Auseinandersetzung mit den eigenen Familiengeschichten und vor allem der eigenen Haltung zu der Thematik auf (vgl. Levy, Sznaider, 2007, S.139f). Doch nicht nur die Serie selbst, auch ihr Titel ,Holocaust' hatten großen Einfluss auf die Geschichte. Durch die Namensgebung

\footnotetext{
${ }^{14}$ https://www.sueddeutsche.de/medien/fernsehen-holocaust-wdr-1.4276735 [Datum des Zugriffs: 30.8.2019]

${ }^{15} \mathrm{https}$ ://www.faz.net/aktuell/feuilleton/medien/serien/ard-sender-wiederholen-die-amerikanischeserie-holocaust-15972921.html [Datum des Zugriffs: 30.8.2019]
} 
und die Etablierung des Begriffes Holocaust „[...] wurde eine neue aktive Phase in der Erinnerungsgeschichte eröffnet." (Assmann, 2018, S.155)

\subsubsection{Die Waldheim-Affäre und ihre Folgen}

Die sogenannte Waldheim-Affäre beschreibt die Debatte, die im Jahr 1986 durch die aktive Wehrmachtsvergangenheit des damaligen Bundespräsidentschaftskandidaten und früheren UN-Generalsekretärs Dr. Kurt Waldheim ausgelöst wurde (vgl. Uhl, 2016, S.92). Ein Artikel in der Zeitschrift ,Profil' von Hubertus Czernin brachte die Debatte ins Rollen. Im März 1986 erschien besagter Artikel, in welchem Czernin Waldheim attestierte, „[...] sich in räumlicher und zeitlicher Nähe zu Nazi-Gräueltaten befunden [...] “16 zu haben. Die ,New York Times“ und der ,World Jewish Congress“ [eine internationale Vereinigung von jüdischen Organisationen, Anm. d. Verf.] griffen die Thematik auf und veröffentlichten Wehrmachtsdokumente, die eine Beteiligung Waldheims an den Verbrechen der Nationalsozialisten beweisen sollten.

Waldheim selbst tätigte dann in der ORF-Pressestunde vom 9. März 1986 eine Aussage, welche in Folge die österreichische Opferthese umstürzen sollte: „Ich habe im Krieg nichts anderes getan als hunderttausende Österreicher auch, nämlich meine Pflicht als Soldat erfüllt." ${ }^{17}$ Es stellte sich heraus, dass Waldheim Mitglied des NSStudentenbundes und des SA-Reiterkorps war, was er in seiner offiziellen Biografie verschwiegen hatte. ${ }^{18}$ Eine Historikerkommission, 1988 eingesetzt, kam zu dem Urteil, dass Waldheim zwar kein Kriegsverbrecher gewesen sei, aber als eingesetzter Dolmetscher dennoch Kenntnis über die Verbrechen hatte.

Waldheim wurde damit zu einem Symbol für die Opferthese Österreichs, für die Verdrängung der eigenen Mitverantwortung. ${ }^{19}$ Die Aussage zur Pflichterfüllung Waldheims verdeutlichte jedoch auch den Widerspruch zur österreichischen Opferthese (vgl. Perz, 2002, S.158). Dr. Kurt Waldheim gewann die Wahl zum

\footnotetext{
${ }^{16} \mathrm{https}: / / w w w . p r o f i l . a t / o e s t e r r e i c h / h i s t o r y / f a l l-k u r t-w a l d h e i m-p f l i c h t-d u n k e l-6253333$ [Datum des Zugriffs: 30.8 .2019$]$

${ }^{17} \mathrm{https} / / /$ derstandard.at/2000031874110/lch-habe-im-Krieg-nichts-anderes-getan-als-meine-Pflicht [Datum des Zugriffs: 30.8 .2019$]$

${ }_{18} \mathrm{http}: / /$ www.demokratiezentrum.org/wissen/wissensstationen/waldheim-debatte.html [Datum des Zugriffs: 30.8 .2019$]$

${ }_{19} \mathrm{https}: / /$ derstandard.at/2000031874110/Ich-habe-im-Krieg-nichts-anderes-getan-als-meine-Pflicht [Datum des Zugriffs: 30.8.2019]
} 
Bundespräsidenten und hatte das Amt von 8.Juli 1986 bis 8.Juli 1992 inne. ${ }^{20}$ Die Haltung Waldheims zu seiner Vergangenheit und deren Verharmlosung hatte jedoch gravierende politische Folgen. Er wurde im Jahr 1987 vom US-Justizministerium auf die ,Watchlist' für vermutete Kriegsverbrecher gesetzt. Damit wurde inm lebenslang die Einreise in die USA verwehrt. ${ }^{21}$ Diskussionen über die mangelnde österreichische Auseinandersetzung bezüglich der eigenen Mitverantwortung an der NSVergangenheit wurden sowohl innenpolitisch als auch in der internationalen Presse geführt (vgl. Uhl, 2016, S.92). Außenpolitisch war Waldheim als Bundespräsident isoliert, es erfolgten kaum Einladungen aus den westlichen Ländern, Bundeskanzler Dr. Franz Vranitzky nahm als Stellvertreter die Stellung des Repräsentanten ein. ${ }^{22}$ Durch die polarisierenden Debatten rund um Kurt Waldheim erstarkte auch die politische Rechte in Österreich. Die Waldheim-Affäre sollte der FPÖ (Freiheitliche Partei Österreichs) unter Dr. Jörg Haider den ersten großen Aufschwung bescheren. ${ }^{23}$

Die politischen Folgen für Österreich, der weltweite Imageverlust und die beginnende Auseinandersetzung mit der „[...] ,Geschichtslüge‘ vom ,ersten Opfer ‘ des Nationalsozialismus [...]" (Uhl, 2016, S.92), wie es Uhl formuliert, führten dazu, dass sich Österreich langsam von der Opferthese trennte.

Bundeskanzler Dr. Franz Vranitzky hielt schließlich am 8. Juli 1991 eine geschichtsträchtige Rede vor dem Nationalrat, in der er „[...] ein Bekenntnis zur österreichischen Mitverantwortung für die Verbrechen des Nationalsozialismus [...]“ (ebd.) ablegte.

\footnotetext{
${ }^{20}$ https://www.parlament.gv.at/WWER/PAD_13961/ [Datum des Zugriffs: 30.8.2019]

${ }^{21} \mathrm{https}: / /$ derstandard.at/2000031874110/lch-habe-im-Krieg-nichts-anderes-getan-als-meine-Pflicht [Datum des Zugriffs: 30.8 .2019$]$

22 https://diepresse.com/home/zeitgeschichte/1342242/Jahrestag_Waldheim-die-SA-sein-Pferd-unddie-Pflicht\#slide-1342242-9 [Datum des Zugriffs: 30.8.2019]

${ }_{23}$ https://www.nachrichten.at/nachrichten/thema/1986-Ein-Jahr-mit-Folgen;art180211,2320366 [Datum des Zugriffs: 30.8.2019]
} 


\subsubsection{Die Stockholm Deklaration}

Einen weiteren Meilenstein in der Erinnerungskultur stellte das ,Stockholm International Forum on the Holocaust' dar (vgl. Uhl, 2016, S.82). Die Konferenz wurde vom 26. bis 28. Jänner 2000 in Stockholm abgehalten. Der Holocaust erlangte damit internationale Bedeutung in der Erinnerungskultur (vgl. Kroh, 2012, S.201). Rund 600 Delegierte aus 46 Ländern nahmen an der Konferenz teil, während der die Stockholm Deklaration verabschiedet wurde (vgl. Uhl, 2016, S.82f).

Das Datum wurde nicht willkürlich gewählt. Erst einige Jahre zuvor, im Jänner 1996, erklärte der deutsche Bundespräsident Roman Herzog den 27.1., den Befreiungstag des Konzentrationslagers Auschwitz Birkenau im Jahr 1945, zum ,Tag des Gedenkens an die Opfer des Nationalsozialismus'. 2005 wurde der 27.1. in der Generalversammlung der Vereinten Nationen zum ,International Holocaust Remembrance Day، erklärt. ${ }^{24}$ Damit wurde dem Artikel 6 der Stockholm Deklaration Folge geleistet, welcher die Verpflichtungserklärung zum Gedenken an den Holocaust, auch durch einen jährlichen Gedenktag, erläutert. ${ }^{25}$

Die Konferenz galt als Schlüsselereignis der Universalisierung des Holocaust (vgl. Uhl, 2016, S.81). Levy und Sznaider sprechen von einer kosmopolitisierten Erinnerung und Gedächtniskultur. Ihrer Ansicht nach entwickelte sich die kosmopolitische Erinnerung durch die negative Erinnerung an den Holocaust. „In der Erinnerung an den Holocaust gewinnt der Bruch mit der Vergangenheit Macht für die Zukunft." (Levy, Sznaider, 2007, S.11) In der Auseinandersetzung mit Schuld entstand ein globaler Rahmen für Gerechtigkeit (vgl. ebd., S.12). „Der souveräne Staat kann nicht mehr die alleinige Grundlage des Rechts und der Gerechtigkeit sein, weil mit dem Holocaust auch die moralischen Grundlagen für globale Institutionen der Gerechtigkeit geschaffen wurden." (ebd.) Eine dieser moralischen Grundlagen war die Verabschiedung der Allgemeinen Erklärung der Menschenrechte der Vereinten Nationen ( $\mathrm{vgl}$. ebd.). Der Holocaust wurde zu einem negativen Gründungsmoment, durch die Entkontextualisierung von Juden und Deutschen zu Menschen wurde ein kosmopolitisches Bewusstsein geschaffen (vgl. Levy, Sznaider,

\footnotetext{
${ }^{24}$ http://www.erinnern.at/bundeslaender/oesterreich/gedenktage/27.-jaenner/27.-jaennerinternationaler-holocaust-gedenktag [Datum des Zugriffs: 30.8.2019]

${ }^{25} \mathrm{https}: / / \mathrm{www}$.holocaustremembrance.com/index.php/de/node/17?usergroup $=7$ [Datum des Zugriffs: 30.8.2019]
} 
2007, S.12). Levy und Sznaider beschreiben diese Entkontextualisierung sehr präzise:

Der Preis der Entkontextualisierung führt auch dazu, daß es in diesem Diskurs keine Juden und keine Deutschen mehr geben darf. Es gibt nur Menschen und Menschheit, wie auch aus dem Begriff der „Verbrechen gegen die Menschheit" und der Entstehung eines moralischen und legalen Kodex gegen „Völkermord“ hervorgeht. (Levy, Sznaider, 2007, S.12f)

Eine kosmopolitische Erinnerung bedeutet, dass sich die Menschen nicht mehr über ihre Nationalität definieren, sondern über ein Wertesystem, das länderübergreifend wirkt. Dieses Wertesystem entstand durch die Erinnerung an den Holocaust, die sich zu einer europäischen, universalen, kosmopolitischen Erinnerung entwickelte (vgl. ebd. S.10ff). Im Rahmen der Konferenz in Stockholm wurde der Holocaust zum ersten Mal durch die Politik „[...] als singuläres historisches Ereignis und Bezugspunkt einer europäischen und potentiell globalen Erinnerungskultur gewürdigt." (Uhl, 2016, S.83) Laut Levy und Sznaider wurde die Stockholm Deklaration zu einer Institution der „[...] entorteten kosmopolitischen Erinnerung.“ (Levy, Sznaider, 2007, S.218)

Der Vollständigkeit halber werden die acht Artikel der Stockholm Deklaration hier aufgelistet:

Wir, die Hohen Vertreter der Regierungen auf dem Stockholmer Internationalen Forum über den Holocaust, erklären Folgendes:

1. Der Holocaust (die Schoah) hat die Zivilisation in ihren Grundfesten erschüttert. In seiner Beispiellosigkeit wird der Holocaust für alle Zeit von universeller Bedeutung sein. Nach einem halben Jahrhundert ist er zeitlich noch hinreichend nah, dass Überlebende Zeugnis ablegen können über 
die Schrecken, die die jüdischen Mitmenschen durchleiden mussten. Das schreckliche Leid der Millionen weiterer Opfer der Nazis hat auch das gesamte Europa mit einer unauslöschlichen Narbe gezeichnet.

2. Das Ausmaß des von den Nazis geplanten und ausgeführten Holocaust muss für immer in unserem kollektiven Gedächtnis verankert bleiben. Die selbstlosen Opfer derjenigen, die sich den Nazis widersetzten und manchmal gar ihr Leben ließen, um Opfer des Holocaust zu schützen oder zu retten, müssen ebenfalls einen festen Platz in unseren Herzen erhalten. Dieses ungeheure Grauen ebenso wie die Größe der Heldentaten können Eckpfeiler für uns sein, die menschliche Fähigkeit zum Guten wie zum Bösen zu verstehen.

3. Da die Menschheit noch immer von Völkermord, ethnischer Säuberung, Rassismus, Antisemitismus und Ausländerfeindlichkeit gezeichnet ist, trägt die Völkergemeinschaft eine hehre Verantwortung für die Bekämpfung dieser Übel. Gemeinsam müssen wir die schreckliche Wahrheit des Holocaust all jenen gegenüber vertreten, die sie bestreiten. Wir müssen die moralische Verpflichtung unserer Völker wie die politische Verpflichtung unserer Regierungen stärken, um sicherzustellen, dass künftige Generationen die Ursachen des Holocaust begreifen können und über seine Folgen nachdenken.

4. Wir verpflichten uns, unsere Anstrengungen zur Förderung der Aufklärung, des Erinnerns und der Forschung im Bereich des Holocaust zu verstärken, und zwar sowohl in den Ländern, die bereits viel in dieser Hinsicht geleistet haben, als auch in denjenigen, die sich unseren Bemühungen anschließen möchten. 
5. Wir haben die gemeinsame Verpflichtung, das Studium des Holocaust in allen seinen Dimensionen anzuregen. Wir werden die Aufklärung über den Holocaust an unseren Schulen und Universitäten sowie in unseren Gemeinden fördern und sie in anderen Einrichtungen unterstützen.

6. Wir haben die gemeinsame Verpflichtung, der Opfer des Holocaust zu gedenken und diejenigen zu ehren, die Widerstand gegen inn geleistet haben. Wir werden geeignete Formen des Erinnerns an den Holocaust in unseren Ländern anregen, darunter einen jährlichen HolocaustGedenktag.

7. Wir haben die gemeinsame Verpflichtung, Licht in das noch immer herrschende Dunkel des Holocaust $z u$ bringen. Wir werden alle erforderlichen Schritte unternehmen, um die Öffnung von Archiven zu erleichtern und somit Forschern den Zugang zu allen Dokumenten mit Bezug zum Holocaust zu gewährleisten.

8. Es ist durchaus angemessen, dass diese erste große internationale Konferenz des neuen Jahrtausends sich dazu bekennt, die Saat einer besseren Zukunft in den Boden einer bitteren Vergangenheit zu streuen. Wir fühlen mit den Opfern, und ihr Kampf ist uns Ansporn. Wir wollen uns verpflichten, der Opfer zu gedenken, die ihr Leben gelassen haben, die noch unter uns weilenden Überlebenden zu achten und das gemeinsame menschliche Streben nach gegenseitigem Verstehen und nach Gerechtigkeit zu bekräftigen. ${ }^{26}$

Die Stockholm Deklaration ist zukunftsweisend formuliert. Das Gedenken richtet sich an die Opfer des Nationalsozialismus und auch an die Personen, die Widerstand

${ }^{26}$ https://www.holocaustremembrance.com/index.php/de/node/17?usergroup=7 [Datum des Zugriffs: 30.8.2019] 
geleistet haben. Die Deklaration enthält jedoch auch Aufrufe an die Gesellschaft, sich zukünftig zu erinnern und sich gegen Rassismus, Antisemitismus und Ausländerfeindlichkeit $\mathrm{zu}$ stellen. Die Deklaration beinhaltet einen sozialpädagogischen Charakter, Forschung und Aufklärung über den Holocaust sollen intensiviert werden. Levy und Sznaider beschreiben die Deklaration als universale Richtlinie, die es möglich macht, sich vom nationalen verankerten Gedächtnis zu lösen. Auch hier zeigt sich die Entortung der Erinnerung (vgl. Levy, Sznaider, 2007, S.220) „Damit wurde der Holocaust offiziell im europäischen Gedächtnis verankert." (ebd.)

\subsubsection{International Holocaust Remembrance Alliance (IHRA)}

In den 1990er Jahren flammte die Debatte um Entschädigungen an die Opfer des Nationalsozialismus wieder auf, vor allem auch in Zusammenhang mit dem Ende der Sowjetunion und des Ostblocks. Die Frage nach Entschädigungen materieller und immaterieller Natur für Jüdinnen und Juden sowie für Zwangsarbeiterlnnen aus Ländern der ehemaligen Sowjetunion und des Ostblocks, die bis dahin nicht berücksichtigt wurden, wurde nun auf internationaler Ebene diskutiert (vgl. Kroh, 2012, S.202f). In den USA entstand die ,World Jewish Restitution Organization' und der Diplomat und Anwalt Stuart Eizenstat wurde von der US-Regierung als Sonderbeauftragter für Vermögensrückgabe in Mittel- und Osteuropa eingesetzt (vgl. ebd. S.203). Es fanden internationale Konferenzen statt, unter anderem die Londoner Nazi-Gold-Konferenz im Jahr 1997 und die Washingtoner Konferenz über geraubte Vermögenswerte im Jahr 1998 (vgl. ebd. S.204). Die Aufarbeitung der Vergangenheit rückte wieder in den Fokus und es zeigte sich, dass diese Aufarbeitung ,[...] - unabhängig vom Ausmaß der historischen Verstrickung mit der Mordpolitik des "Dritten Reiches“ - in- und außerhalb Europas zur Pflicht geworden war." (ebd.)

Diesbezüglich zeigte auch der schwedische Premierminister Göran Persson großes Engagement. Er initierte den Ausbau der sogenannten ,Holocaust-Education: Aufgrund seines Engagements wurde am 7. Mai 1998 in Stockholm das ,Stockholm Meeting on the Holocaust' abgehalten. Während dieser Konferenz wurde die ,Task Force for International Cooperation on Holocaust Education, Remembrance, and 
Research (ITF)' ins Leben gerufen, welche in weiterer Folge im Jahr 2012 in IHRA ,International Holocaust Remembrance Alliance' umbenannt wurde (vgl. Kroh, 2012, S.206).

Auf der Holocaust-Konferenz in Stockholm im Jahr 2000 wurde „[...] die ITF als relevanter internationaler Akteur wahrgenommen [...]" (ebd., S.211), was sich vor allem am breiten Interesse der teilnehmenden Staaten an einer Mitgliedschaft in der ITF widerspiegelte.

Assmann schreibt zur IHRA, diese habe „[...] zur praktischen Umsetzung einer europäischen Erinnerungskultur beigetragen, die für alle Mitgliedsstaaten verpflichtend ist." (Assmann, 2016a, S.156) Der IHRA gehören heute 33 Mitgliedsstaaten an, der Vorsitz wechselt jährlich. ${ }^{27}$ Österreich ist seit 2001 aktives Mitglied und hatte den Vorsitz von März 2008 bis März 2009 inne. Der Nationalfonds ist die österreichische Koordinierungsstelle für die IHRA. LeiterInnen der österreichischen Delegation sind Mag. Hannah Lessing und Dr. Thomas Michael Baier. ${ }^{28}$ Die IHRA setzt sich international für Forschung und Bildung auf dem Gebiet der Holocaust-Erziehung ein und fördert in diesem Zusammenhang auch Projekte. ${ }^{29}$ Am 11. März 2008 wurde in Berlin das ,permanent office - die feste Geschäftsstelle - der IHRA eingerichtet. Das Büro organisiert die jährlichen Konferenzen, unterstützt die jeweiligen Vorsitzländer und dient den Mitgliedsstaaten als zentrale Vermittlungsstelle. ${ }^{30}$

Eine Arbeitsdefinition zur Leugnung und Verharmlosung des Holocaust wurde im Jahr 2013 von der IHRA verabschiedet. Dieser rechtlich nicht bindenden Definition „[...] liegt die Erkenntnis zugrunde, dass die Leugnung und Verfälschung des Holocaust sowohl national als auch international bekämpft, geächtet und weltweit untersucht werden müssen. ${ }^{\text {“31 }}$ Dieser Arbeitsdefinition folgend wurde ein Dossier von der IHRA verfasst, welches laufend aktualisiert wird und als Anleitung dienen soll. ${ }^{32}$

\footnotetext{
${ }^{27}$ https://www.holocaustremembrance.com/our-structure [Datum des Zugriffs: 30.8 .2019 ]

${ }^{28} \mathrm{https}: / / \mathrm{www}$.holocaustremembrance.com/member-countries/austria [Datum des Zugriffs: 30.8 .2019$]$

${ }^{29} \mathrm{https}: / / \mathrm{www}$. nationalfonds.org/ihra.html [Datum des Zugriffs: 30.8 .2019 ]

${ }^{30} \mathrm{https}: / / \mathrm{www}$.holocaustremembrance.com/index.php/permanent-office [Datum des Zugriffs: 30.8.2019]

${ }^{31}$ https://holocaustremembrance.com/de/node/122 [Datum des Zugriffs: 30.8 .2019 ]

${ }^{32} \mathrm{https} / / /$ holocaustremembrance.com/sites/default/files/inline-files/Paper\%20on\%20Distortion_0.pdf [Datum des Zugriffs: 30.8.2019]
} 
Im Jahr 2016 wurde das Plenum der IHRA vom Ausschuss für Antisemitismus und Holocaustleugnung aufgefordert, eine Arbeitsdefinition von Antisemitismus anzunehmen. Die nicht rechtsverbindliche Arbeitsdefinition wurde am 26. Mai 2016 angenommen und lautet wie folgt:

Antisemitismus ist eine bestimmte Wahrnehmung von Juden, die sich als Hass gegenüber Juden ausdrücken kann. Der Antisemitismus richtet sich in Wort und Tat gegen jüdische oder nicht-jüdische Einzelpersonen und / oder deren Eigentum, sowie gegen jüdische Gemeindeinstitutionen und religiöse Einrichtungen. ${ }^{33}$

Der österreichische Ministerrat hat diese Arbeitsdefinition am 21. April 2017 angenommen. Sie stellt die erste einheitliche Definition von Antisemitismus in Österreich dar und soll dabei helfen, Antisemitismus $\mathrm{zu}$ identifizieren und $\mathrm{zu}$ bekämpfen. Die Arbeitsdefinition kann ebenfalls als Leitfaden für Gesetzesmaßnahmen gelten, sowie im Bildungsbereich und in der Vermittlungsarbeit herangezogen werden. ${ }^{34}$

\subsection{Kritik zu Erinnerungs- und Gedenkkultur}

Erinnerungskultur wird in ihrer Veränderung auch immer wieder kritisch betrachtet. Assmann knüpft in ihrem Buch ,Das neue Unbehagen an der Erinnerungskultur' an Sigmund Freuds Buch ,Das Unbehagen in der Kultur' aus dem Jahre 1930 an. Laut Freud ist die Schuld „[...] zur Grundlage des individuellen Gewissens geworden [...]" (Assmann, 2016a, S.9). Erinnerungskultur ist somit ein Ergebnis der Schuld, die durch Rituale am Leben erhalten wird. Grundlage dafür ist ein negatives Gedächtnis, welches „[...] die eigene verbrecherische Vorgeschichte ins kollektive Selbstbild integriert und durch öffentliches Bekennen von Schuld rituell in Gang hält." (ebd.)

\footnotetext{
${ }^{33}$ https://www.holocaustremembrance.com/de/node/196 [Datum des Zugriffs: 30.8.2019]

${ }^{34} \mathrm{http}: / /$ www.erinnern.at/bundeslaender/oesterreich/e_bibliothek/antisemitismus-1/was-istantisemitismus-oesterreich-nimmt-ihra-arbeitsdefinition-von-antisemitismus-an [Datum des Zugriffs: 30.8.2019]
} 
Doch diese sogenannte Ritualisierung ruft auch Kritik hervor. Laut Assmann gibt es drei Komponenten der Kritik: „Emotionalisierung (Pathos der Betroffenheit), Inszenierung (leere rituelle Wiederholungen) und Institutionalisierung (Festschreibung der Erinnerung für die Zukunft)." (Assmann, 2016a, S.77) Dennoch ließe sich aus diesen drei Komponenten ebenso die Qualität der kollektiven Erinnerungskultur ableiten (vgl. ebd.). Eine Ritualisierung wäre vor allem in der staatlichen Erinnerungskultur festzustellen. Aufgrund der Präsenz der offiziellen, staatlichen Erinnerungskultur könne der Eindruck entstehen, dass Erinnerungskultur nur auf dieser Ebene stattfinden würde, was ein Unbehagen an eben diesen Ritualen zur Folge hätte. Dieser Eindruck sei jedoch falsch. Es gäbe durchaus eine Vielfalt an zivilgesellschaftlichen Auseinandersetzungen mit Erinnerungskultur, wie Assmann erläutert (vgl. ebd., S.78).

Assmann erläutert weiters, dass das Wissen um den Holocaust mittlerweile allgegenwärtig sei. Nach Meinung der Kritiker laufe „[...] der anhaltende Erinnerungsimperativ und das Vergessensverbot ins Leere." (Assmann 2016b, S.33) Auch Radonić und Uhl halten fest, dass die Entstehung einer Erinnerungskultur und die damit verbundene Aufwertung des Erinnerns einen Kampf gegen das Vergessen darstellen würden (vgl. Radonić, Uhl, 2016, S.9).

Die Fixierung auf die Vergangenheit würde eine Orientierung für die Zukunft erschweren. Kritiker würden sich dafür aussprechen, mit der Erinnerung abzuschließen (vgl. Assmann, 2016b, S.34). Assmann selbst jedoch betont die Wichtigkeit des Kontextes. „Ob Erinnern gut oder schlecht ist, das entscheidet aber erst der Kontext, in dem erinnert wird." (ebd., S.31)

Für Hermann Düringer ist die Reduktion von Erinnerung auf eine pädagogische und politische Funktion problematisch. Er sieht darin eine Instrumentalisierung für Lernmaterial. Erinnerung solle den Opfern selbst dienen und keinen anderen Zweck verfolgen (vgl. Düringer, 2012, S.66).

Kritik wird jedoch nicht nur der Erinnerungskultur an sich, sondern auch den Gedenkstätten gegenüber ausgesprochen. Ruth Klüger, selbst eine Überlebende des Konzentrationslagers Auschwitz-Birkenau, empfindet die Transformation der 
Konzentrationslager zu Museen als kritisch. „Es ist unsinnig, die Lager räumlich so darstellen zu wollen, wie sie damals waren. Aber fast so unsinnig ist es sie mit Worten beschreiben zu wollen, als liege nichts zwischen uns und der Zeit, als es sie noch gab.“ (Klüger, 2008, S.98) Sie führt weiter aus: „Nach Auschwitz bin ich nicht zurückgegangen und habe auch nicht die Absicht, es in diesem Leben noch zu tun. Mir ist Auschwitz kein Wallfahrtsort, keine Pilgerstätte." (ebd. S.173) Sie bezeichnet die KZ-Gedenkstätten als „[...] Antimuseen mit Todesaura [...].“ (Knigge, 2002, S.378) In ihrer Rede zum Gedenktag im Jahr 2011 im Parlament hält Ruth Klüger fest: "Weder andächtiges Schweigen noch Reue, Andacht oder auch Hass und Verachtung geben uns Antwort auf die Fragen, die die Geschichte des vergangenen Jahrhunderts uns stellt. Und so belassen wir es bei der festen Überzeugung, dass jetzt alles anders ist.“ 35

Dr. Christian Angerer, pädagogischer Mitarbeiter der KZ-Gedenkstätte Mauthausen, beschäftigt sich in einem Artikel ebenfalls mit der Frage der Funktion der Gedenkstätten. Für inn sollen diese Orte „[...] zum Austausch über Wahrnehmungen, Gefühle und Gedanken[...] ${ }^{436}$ anregen. Eine kritische Auseinandersetzung hat dennoch auch inm zufolge ihre Berechtigung. „NS-Geschichte ausstellungspädagogisch gezähmt und verpackt zum Lernen für die Zukunft? Ein verlockender Gedanke, doch ich zweifle, ob sich diese Geschichte dem rationalen Zugriff fügt. Jedenfalls tut sie es nicht an den Gedenkstätten, die Überreste der Mordstätten bewahren. ${ }^{37}$

Auch der deutsche Publizist Henryk Broder kritisiert die KZ-Gedenkstätte Auschwitz als ,Disneyland des Todes' und ,Wellnessoase der Vergangenheitsbewältigung ' ${ }^{38}$

Dr. Harald Welzer, ein deutscher Soziologe und Sozialpsychologe, spricht sich für eine Renovierung der Erinnerungskultur aus. In einem Gedenkstättenrundbrief aus dem Jahr 2011 schreibt er über das Geschichtsbewusstsein der Jugend, das in einem Paradoxon münden würde. Obwohl kaum jemand die Bedeutung der

\footnotetext{
${ }^{35}$ https://www.parlament.gv.at/ZUSD/PDF/Gedenktagsrede_RuthKlueger.pdf [Datum des Zugriffs: 30.8.2019]

${ }^{36}$ https://www.ph-online.ac.at/ph-ooe/voe_main2.getVollText?pDocumentNr=484712\&pCurrPk=5524

[Datum des Zugriffs: 30.8.2019]

37 ebd.

${ }^{38} \mathrm{https}$ ://www.focus.de/politik/deutschland/henry-m-broder-kritisiert-vergangenheitsbewaeltigungauschwitz-ist-ein-disneyland-des-todes_aid_722898.html [Datum des Zugriffs: 30.8.2019]
} 
Vermittlung der Geschichte des Nationalsozialismus und des Holocaust anzweifeln würde, so würde dennoch ein Gefühl von Zwang, sich erinnern zu müssen, entstehen. Er schreibt: „Aus sozialpsychologischer Sicht ist es ohnehin kontraproduktiv, die Vermittlung historischen Wissens mit einer moralischen Gebrauchsanweisung zu versehen. Aktive Aneignungsprozesse und die Entwicklung eigenständiger Deutungen und Bewertungen sind viel nachhaltiger für das Geschichtsbewusstsein als die Übernahme vorgefertigter moralistischer Glaubenssätze. “39 Er fordert eine Modernisierung der Erinnerungskultur mit Bezug zur Gegenwart. Eine Öffnung der Erinnerungskultur hin zu einer aktuellen gesellschaftspolitischen Diskussion über Ausgrenzung würde einen Anschluss an die Lebenswelt von Jugendlichen aus anderen Kulturen ermöglichen. „Wenn die um den Holocaust zentrierten erinnerungskulturellen Anstrengungen einen Sinn haben können, dann doch offensichtlich den, begreiflich zu machen, dass unter bestimmten Bedingungen sich nicht nur die bösen Menschen zu gegenmenschlichem Verhalten entscheiden, sondern auch die guten." (Welzer, 2012, S.39) Die Erinnerungskultur stehe vor der Herausforderung, eben diese Gegenwartsbezüge herzustellen, um an aktuelle zivilgesellschaftliche Entwicklungen anschließen zu können (vgl. ebd.).

Auch Assmann nimmt Bezug auf Welzer und erläutert, dass es nicht darum ginge, „[...] ob Erinnern an sich gut oder schlecht ist - die Frage ist in dieser Form gar nicht beantwortbar -, sondern darum, wie das Erinnern jeweils neu zu füllen und zu aktualisieren ist." (Assmann, 2016a, S.72)

\subsection{Die Zukunft der Erinnerungskultur}

Die Diskussion über und der Umgang mit einer zukünftigen Erinnerungskultur steht seit einigen Jahren an einer erneuten Wende. Diskussionen über eine zukünftige Erinnerungskultur schließen an den zuvor beschriebenen Kritikpunkten an. Im folgenden Kapitel wird erläutert, welche Überlegungen zu einer erfolgreichen Zukunft der Erinnerungskultur in der Fachwelt bereits bestehen. Im Fokus steht diesbezüglich auch die Frage nach einer Zukunft der Erinnerungskultur ohne Zeitzeuglnnen.

\footnotetext{
${ }^{39} \mathrm{https}: / /$ www.gedenkstaettenforum.de/nc/aktuelles/einzelansicht/news/fuer_eine_modernisierung_der _erinnerungs_und_gedenkkultur/ [Datum des Zugriffs: 30.8.2019]
} 
Sabrow bezeichnet die Zeitzeuglnnen als Wanderer zwischen den Welten des Gestern und Heute.

Um als Wanderer zwischen diesen Welten dienen zu können, übernimmt er von der Vergangenheit die Erinnerung, von der Gegenwart aber die Wertmaßstäbe, das kulturelle Rahmenformat, in dem er das Vergangene memoriert und zugleich aktualisiert. Im Zeitzeugen kommen die beiden kulturellen Hauptströmungen unseres Geschichtsdenkens zur Deckung, und eben darin liegt seine tiefste Anziehungskraft begründet. (Sabrow, 2012, S.27)

Schneider stellt in diesem Zusammenhang die Frage, welche Veränderungen ein Fehlen der ZeitzeugInnen-Berichte für die Erinnerungskultur bedeuten würde. Für inn markiert dies den „[...] Übergang vom kommunikativen zum kulturellen Gedächtnis [...]." (Schneider, 2012, S.85) Dieser Übergang habe „[...] stets den Charakter einer Krise." (ebd.) Frei hingegen widerspricht dieser Gegenüberstellung der Gedächtnistheorien. Für inn wäre es „[...] im Blick auf die lange Geschichte des Umgangs mit der NS-Vergangenheit präziser, von Generationen-Gedächtnissen zu sprechen und von deren sich verändernden Relationen zueinander." (Frei, 2002, S.372)

Für Welzer ist die Zukunft der Erinnerungskultur fraglich. Eine Prognose zukünftiger Erinnerungen sei „[...] ein waghalsiges Unterfangen.“ (Welzer, 2002, S.342). Laut Welzer kann man nicht vorhersagen, wie die Erinnerung an den Holocaust in 50 Jahren aussehen wird. Ihm zufolge haben die Menschheitsverbrechen des 20. Jahrhunderts zwei Einsichten hervorgerufen:

Die erste besteht darin, daß katastrophale und genozidale Entwicklungen keine Betriebsunfälle eines ansonsten linear fortschreitenden Zivilisierungsprozesses sind, sondern Potentiale, die mit seinem Fortschreiten anwachsen; die zweite, daß gesellschaftliche Entwicklungen, mit denen niemand gerechnet hat, sich in ungeheurer Geschwindigkeit vollziehen 
können. Letzteres gilt für die Dynamik, mit der die nationalsozialistische Gesellschaft ihre Option auf die Durchführung des größten Verbrechens der Geschichte entwickelt und durchgesetzt hat [...]. (Welzer, 2002, S.342)

Ausgehend von dem sich verändernden Generationen-Gedächtnis, wie von Frei beschrieben, deutet auch Welzer eine zukünftige Abnahme in der Intensität der Erinnerung an. Er schreibt, dass „[...] mit dem Heranwachsen der vierten und fünften Generation nach dem Holocaust die unmittelbare generationelle Verbindung zu diesem historischen Geschehenszusammenhang verschwindet." (Welzer, 2012, 35) In diesem Zusammenhang betont Assmann jedoch, dass Welzer die transgenerationelle Weitergabe von Traumatisierungen außer Acht lässt (vgl. Assmann, 2016a, S.102).

Für Welzer besteht durchaus die Möglichkeit, dass ,men made disasters' [von Menschen verursachte Traumen, Anm. d. Verf.], wie der Holocaust, erneut möglich sein werden, eventuell in noch größerem Ausmaß.

Falls der Holocaust eine Lehre beinhaltet, dann die, daß alles möglich ist, daß die zivilisatorischen Rückhaltesysteme gegenüber maßstabsloser Inhumanität viel schwächer sind, als man zuvor zu befürchten gewagt hat und, so glaube ich, auch im nachhinein [sic] noch zu befürchten wagt. (Welzer, 2002, S.343)

Für inn zeigt sich die Problematik der aktuellen Erinnerungskultur „[...] in der immer deutlicher werdenden Petrifizierung des zu Vermittelnden, im Ritual- und Floskelhaften seiner Kommunikation und in der Wiederholung des Immergleichen." (Welzer, 2012, S.36) Diese Art der Vermittlung würde den Zwang beinhalten, verstehen zu müssen. Welzer spricht sich dafür aus, dass eine Orientierung an bestehenden Wirklichkeiten erfolgen muss und Vermittlungsarbeit in Zukunft nicht einfach eine Wiederholung der geschichtlichen Fakten darstellt, sondern Raum für Diskussionen zu Vorwissen und Vorstellungen bietet (vgl. ebd.). 
Welzer bescheinigt der erinnerungskulturellen Pädagogik einen hybriden Charakter, der sich darauf begründet, „[...] dass sich die Betrachtung des Vernichtungsprozesses vor allem auf das Resultat, also den Holocaust, konzentriert, und nicht auf seine Genese, die verblüffend schnelle Formatierung einer Ausgrenzungsgesellschaft." (Welzer, 2012, S.37)

Seiner Meinung nach muss sich eine erfolgreiche Erinnerungskultur neu definieren und den Blick auf die (durchaus auch wieder aktuelle) Thematik der Ausgrenzungsgesellschaft und wie eine solche entstehen kann, richten (vgl. ebd., S.38f).

Seine Überlegungen gründen unter anderem auf der Tatsache, dass die heutige Jugend und Gesellschaft heterogener als noch in den 1980er Jahren sind. Jugendliche mit Migrationshintergrund bilden eine neue Zielgruppe, für die neue Vermittlungsansätze notwendig sind. Welzer nennt in diesem Zusammenhang als Beispiel eventuelle Gewalterfahrungen, die Aktualisierungschancen für neue Ansätze der Erinnerungskultur beinhalten könnten (vgl. ebd., S.44). Erinnerungskultur würde einem Wandel unterliegen, der erste Wandel sei durch den opferorientierten Perspektivenwechsel geschehen, der die jetzige Erinnerungskultur präge. Nun stehe die Erinnerungskultur erneut vor einem Wandel, in dem es gelingen müsse, „[...] sich den Potentialen, Handlungen und Orientierungen $\mathrm{zu}$ widmen, die Ausgrenzungsgesellschaften entstehen und Genozide möglich werden lassen. Genau in diesem Sinn ist Erinnerungskultur eine zivilgesellschaftliche Angelegenheit, deren Bezugspunkt die Gegenwart und nicht die Vergangenheit ist." (ebd., S.43)

Auch Uhl beschreibt den erneuten Wandel, dem die Erinnerungskultur unterliegt. Laut inr ist die Erinnerung an den Holocaust nun nicht mehr „[...] durch Verdrängen und Verschweigen bedroht, sondern durch den Verlust an Relevanz und sozialer Energie." (Uhl, 2012, S.244)

Durch eine Reflexion der Erinnerungskultur, die Anknüpfung an die Gegenwart, an aktuelle Lebenswelten, an die heutige Gesellschaft und an aktuelle Ausgrenzungsmechanismen könnten auch kommende Generationen an die Thematik anschließen (vgl. ebd. S.44ff). In diesem Kontext sieht Welzer „[...] den 
Verlust an Zeitzeugen [...] aber nicht als Problem, sondern als große Chance für eine Neuausrichtung der Erinnerungs- und Bildungsarbeit [...]." (Welzer, 2012, S.48)

Assmann nimmt ebenfalls Bezug auf das Unbehagen, das die Erinnerungskultur derzeit auslöse. Durch die erfolgreiche Etablierung der Erinnerungskultur wäre mittlerweile ein Überdruss in der Gesellschaft entstanden, der zu Abwehrhaltungen führen könne. In diesem Kontext bezieht sie sich auf die Ausführungen von Welzer. Bezüglich seines Ansatzes, die Erinnerungskultur in ihrer jetzigen Form als erledigt zu betrachten und sich neuen Aufgaben zuzuwenden, dürfe man laut Assmann jedoch nicht vergessen, dass die Erinnerung an den Holocaust zusätzlich zum historischen Wissen „[...] noch einen Identitätsbezug aufweist, der dieses Ereignis mit der Entstehung unseres Staates [gemeint ist Deutschland, Anm. d. Verf.], eigenen Familiengeschichten und einer ethischen Orientierung verknüpft." (Assmann, 2016a, S.72) Dieser Identitätsbezug sei die Form des kulturellen Gedächtnisses, welcher, wenn man Welzers Ausführungen zu einem Abschluss der Erinnerungskultur folgen würde, aufgelöst werden würde (vgl. ebd.).

Assmann ist der Meinung, „[...] dass sich Erinnerungskultur und Historisierung, Zukunft und Vergangenheit nicht gegenseitig ausschließen, sondern zwei legitime und komplementäre Formen des Vergangenheitsbezugs sind." (ebd., S.105)

Viele Modernisierungstheorien würden den bleibenden Identitätsbezug ignorieren. Assmann misst dem genannten historischen Identitätsbezug große Bedeutung bei. „Der historische Identitätsbezug hat sich inzwischen zu einer transnationalen Erinnerung ausgeweitet, die dabei auch andere Tore zur Vergangenheit aufgestoßen hat und es ermöglichte, dass bislang zum Schweigen verurteilte Gewaltgeschichten ebenfalls artikuliert wurden und Gehör finden konnten." (ebd. S.106) Assmann schreibt der Erinnerungskultur Ressourcen für die Gegenwart zu.

Die Wiederentdeckung der anthropologischen, sozialen und kulturellen Bedeutung von Erinnerung hat $\mathrm{zu}$ einer wichtigen Erweiterung des Erinnerungsbegriffs vom Individuum auf Gruppen und von Gruppen auf kollektive Akteure geführt und damit zugleich zu seiner Ausdehnung auf 
Medien, Speichertechniken und Institutionen im Rahmen eines kulturellen Gedächtnisses. (Assmann, 2016a, S.204)

Welch große Bedeutung Erinnerungskultur auch in der Zukunft haben sollte, wird anhand einer aktuellen Studie der Claims Conference (Conference on Jewish Material Claims against Germany), einer Organisation, die sich seit 1951 für Entschädigung für Holocaust-Opfer und auch für Vermittlungsarbeit und soziale Hilfestellungen einsetzt, ersichtlich. ${ }^{40}$ Die Ergebnisse der Studie zum Wissen über den Holocaust in Österreich wurden im Mai 2019 veröffentlicht. Beispielhaft werden einige Ergebnisse hier genannt. „Mehr als die Hälfte der Befragten (56\%) wusste nicht, dass während des Holocaust sechs Millionen Juden ermordet wurden. Unter den um die Jahrtausendwende Geborenen und der Generation Z waren es sogar 58 Prozent. “41 Auf die Frage nach bekannten Konzentrationslagern konnten $42 \%$ der Befragten das österreichische Konzentrationslager Mauthausen nicht nennen. Weitere Fragen bezogen sich auf die Opferthese Österreichs, die Anzahl der Neonazis in Österreich oder auf die Wahrscheinlichkeit, mit der ein neuerlicher Holocaust möglich sei. $82 \%$ der Befragten sprachen sich für einen verpflichtenden Unterricht in den Schulen über den Holocaust aus, $75 \%$ für ein Beibehalten der Vermittlungsarbeit. ${ }^{42}$

\footnotetext{
${ }^{40} \mathrm{https} / / /$ www.claimscon.de/ueber-uns.html [Datum des Zugriffs: 30.8.2019]

${ }^{41} \mathrm{https}: / /$ www.claimscon.de/no_cache/presse-news/aktuelles/newsdetail.html?tx_news_pi $1 \% 5 \mathrm{Bnews} \% 5 \mathrm{D}=58 \mathrm{ktx}$ news_pi $1 \% 5 \mathrm{~B}$ controller\%5D=News\&tx_news_pi $1 \% 5 \mathrm{~B}$ action\%5D=detail\&cHash=4f7002a76dc756505ce0173a66e38a42 [Datum des Zugriffs: 30.8 .2019 ] ${ }^{42}$ vgl. ebd.
} 


\section{Nationalfonds der Republik Österreich für Opfer des Nationalsozialismus}

In den folgenden Kapiteln wird die Gründung des Nationalfonds beschrieben und die vielfältigen Aufgabengebiete näher erläutert. Um einen vollständigen Überblick zu geben, werden neben dem Nationalfonds auch der Allgemeine Entschädigungsfonds für Opfer des Nationalsozialismus und der Fonds zur Instandsetzung der jüdischen Friedhöfe in Österreich, welche sich die Büroräumlichkeiten mit dem Nationalfonds teilen und sowohl administrativ als auch personell eng mit dem Nationalfonds verbunden sind, beschrieben.

\subsection{Die Gründung des Nationalfonds}

Die Gründung des Nationalfonds fällt in die Zeit des aufkommenden Bedürfnisses in der Gesellschaft Österreichs, sich mit der Vergangenheit auseinander zu setzen. Die Abkehr von der österreichischen Opferthese und die aufkommenden Fragen zu einer gerechten Entschädigungspolitik waren dafür ausschlaggebend. Eine Bürgerinitiative von Personen, die aus dem sogenannten Döllersheimer Ländchen ausgesiedelt worden waren und für ihre erzwungene Aussiedelung keine Entschädigung erhalten hatten, legte den Grundstein für die Errichtung des Nationalfonds. Das Döllersheimer Ländchen befand sich auf dem Gebiet, auf dem der Truppenübungsplatz Allentsteig errichtet wurde. Der Ausschuss für Petitionen und Bürgerinitiativen hat die ausgesiedelten Personen als Opfer des Nationalsozialismus anerkannt und einen Antrag an den Nationalrat gestellt. „Es sollen daher Wege gesucht werden, um allen Personen, die Opfer des Nationalsozialismus sind und für das innen widerfahrene Leid nicht entschädigt wurden, auf möglichst unbürokratische und menschliche Art und Weise zu helfen." (Stenographisches Protokoll der 173. Sitzung des Nationalrates der Republik Österreich, 1994, S.63) So formulierte es die Berichterstatterin Christine Haager in der Nationalratssitzung vom 16. Juli 1994, nachzulesen in den stenographischen Protokollen der Sitzung. Es folgte ein Entschließungsantrag über die „Errichtung eines Nationalfonds für Opfer des Nationalsozialismus aus Anlaß des 50. Jahrestages der zweiten Republik." (ebd., S.64) Der Antrag sah Entschädigungsleistungen und Hilfe für die Opfer direkt vor sowie Leistungen, die Opfer in Form von Projekten unterstützen sollten (vgl. ebd.). 
Der Antrag zur Errichtung des Nationalfonds wurde an diesem Tag mehrheitlich beschlossen (vgl. Stenographisches Protokoll der 173. Sitzung des Nationalrates der Republik Österreich, 1994, S.70). In derselben Sitzung brachte die FPÖ einen weiteren Antrag ein, der die „[...] Errichtung eines Nationalfonds für Opfer der Gewalt im schrecklichen Jahrzehnt von 1938 bis 1948 [...]" (ebd. S.67) vorsah, dieser Antrag wurde jedoch mehrheitlich abgelehnt (vgl. ebd., S.70).

Dr. Volker Kier, damaliger Abgeordneter des Liberalen Forums, unterstrich in der Nationalratssitzung vom 1. Juni 1995 die Bedeutung des Nationalfonds:

Wir haben die einmalige Chance, durch diesen Fonds, den wir mit Zweidrittelmehrheit ausstatten, in den höchsten Rang unserer Verfassung heben und damit gleichzeitig - das sage ich ganz bewußt - auch gegen die leichtfertige Abschaffung absichern, immer wieder aufs neue [sic] unter Beweis zu stellen, daß wir wissen, daß wir 50 Jahre lang die Sühnebereitschaft schuldig geblieben sind. Es ist kein Fonds, der sich dafür eignet, zu sagen: Jetzt ist das Problem endlich erledigt, jetzt ist Ende der Diskussion! Es wäre ja auch geradezu zynisch gewesen, [...] das Ende der Diskussion zu verkünden, einer Diskussion, die in diesem Land bekanntlich gar nicht oder nur rudimentär stattgefunden hat, in einem Land, in dem die Verdrängung geradezu ein konstitutives Merkmal unserer Gesellschaft ist [...]. (Stenographisches Protokoll der 40. Sitzung des Nationalrates der Republik Österreich, 1995, S.71)

Die Verabschiedung des Bundesgesetzes über den Nationalfonds der Republik Österreich für Opfer des Nationalsozialismus erfolgte schließlich am 1. Juni 1995 durch den Nationalrat (vgl. Lessing, 2000, S.67). 
Das Gesetz trat rückwirkend mit 27. April 1995 in Kraft. ${ }^{43}$ „Der Nationalfonds wurde [...] bei der Parlamentsdirektion eingerichtet, um seine Bedeutung zu unterstreichen [...]." (Meissner, 2005b, S.13)

Im Paragraph 2 des Bundesgesetzes über den Nationalfonds werden die Voraussetzungen der Zuerkennungen der Leistungen beschrieben:

§ 2. (1) Der Fonds erbringt Leistungen an Personen,

1. die vom nationalsozialistischen Regime aus politischen Gründen, aus Gründen der Abstammung, Religion, Nationalität, sexuellen Orientierung, auf Grund einer körperlichen oder geistigen Behinderung oder auf Grund des Vorwurfes der sogenannten Asozialität verfolgt oder auf andere Weise Opfer typisch nationalsozialistischen Unrechts geworden sind oder das Land verlassen haben, um einer solchen Verfolgung zu entgehen, und 2. die

a) am 13. März 1938 die österreichische Bundesbürgerschaft und einen Wohnsitz in Österreich oder

b) bis zum 13. März 1938 durch etwa zehn Jahre hindurch ununterbrochen ihren Wohnsitz in Österreich gehabt haben bzw. in diesem Zeitraum als Kinder von solchen Personen in Österreich geboren wurden oder

c) vor dem 13. März 1938 die österreichische Bundesbürgerschaft oder ihren zumindest etwa zehnjährigen Wohnsitz verloren haben, weil sie wegen des unmittelbar bevorstehenden Einmarsches der Deutschen Wehrmacht das Land verlassen haben, oder

${ }^{43}$ https://www.ris.bka.gv.at/Dokumente/BgblPdf/1995_432_0/1995_432_0.pdf [Datum des Zugriffs: 30.8.2019] 
d) vor dem 9. Mai 1945 als Kinder von solchen Personen im Konzentrationslager oder unter vergleichbaren Umständen auch in Österreich geboren worden sind.

(2) Leistungen werden insbesondere an Personen vergeben, die keine oder eine völlig unzureichende Leistung erhielten, die in besonderer Weise der Hilfe bedürfen oder bei denen eine Unterstützung auf Grund ihrer Lebenssituation gerechtfertigt erscheint.

(3) Der Fonds kann auch Projekte unterstützen, die Opfern des Nationalsozialismus zugute kommen [sic], der wissenschaftlichen Erforschung des Nationalsozialismus und des Schicksals seiner Opfer dienen, an das nationalsozialistische Unrecht erinnern oder das Andenken an die Opfer wahren.

(4) Der Fonds erbringt einmalige oder wiederkehrende Geldleistungen. Nähere Vorschriften über die Leistungen können in Richtlinien des Fonds erlassen werden. ${ }^{44}$

Wie bereits im Entschließungsantrag formuliert, sollte die Hilfe für die Opfer des Nationalsozialismus rasch und unbürokratisch gewährt werden können. Um dies zu ermöglichen, „[...] war es notwendig, davon abzusehen, einen Rechtsanspruch auf Leistungen einzuräumen, weil dann im Gesetz genaue Tatbestände, Leistungsvoraussetzungen und Leistungshöhe vorgeschrieben hätten werden müssen, deren Vorliegen im Einzelfall in einem Verwaltungsverfahren nachzuweisen gewesen wäre." (Lessing, 2000, S.68)

${ }^{44}$ https://www.ris.bka.gv.at/Dokumente/BgblPdf/1995_432_0/1995_432_0.pdf [Datum des Zugriffs: 30.8.2019] 


\subsection{Die organisatorische Struktur des Nationalfonds}

Die Organe des Nationalfonds werden im Nationalfondsgesetz [im folgenden NF-G genannt, Anm. d. Verf.] unter Paragraph 3 festgeschrieben und bestehen aus Kuratorium, Komitee und Generalsekretär. ${ }^{45}$

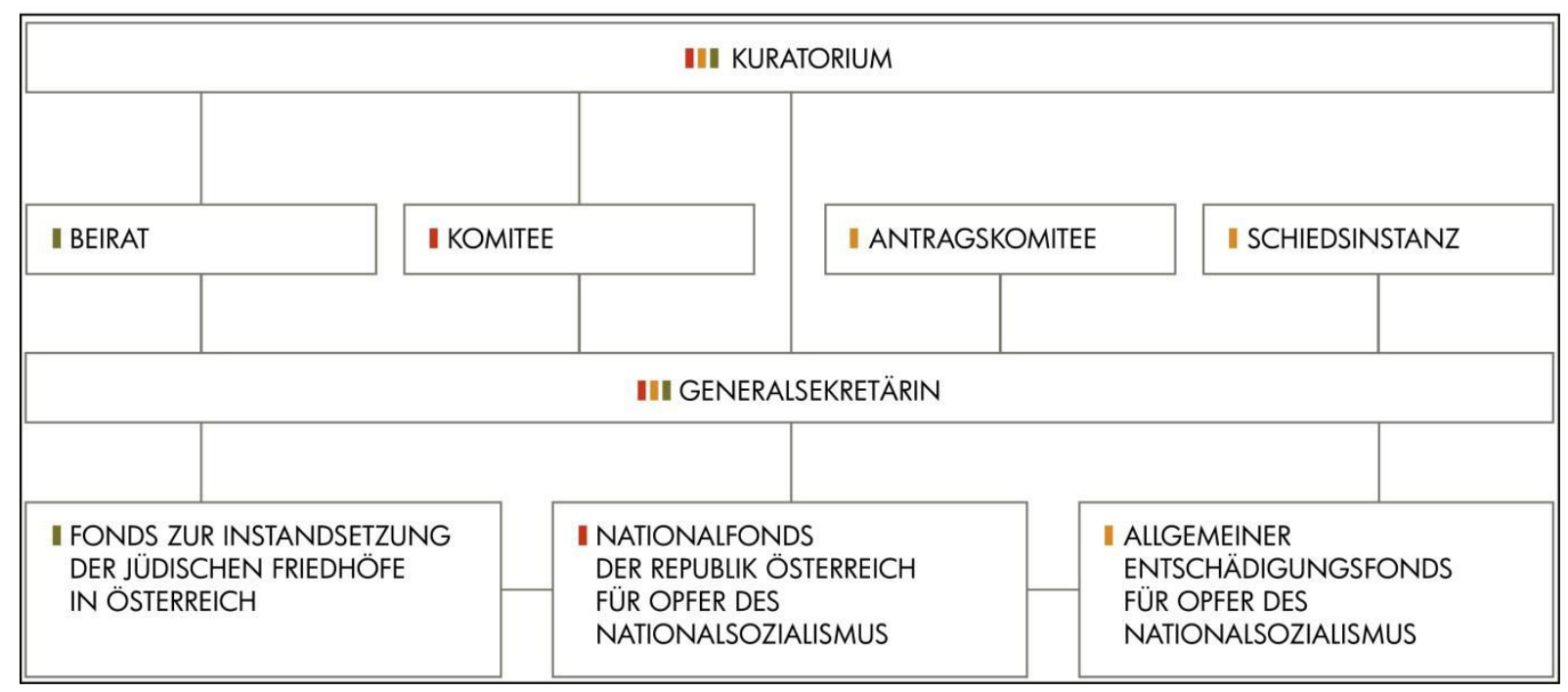

Abb.1: Die Organe des Nationalfonds der Republik Österreich für Opfer des Nationalsozialismus, des Allgemeinen Entschädigungsfonds für Opfer des Nationalsozialismus und des Fonds zur Instandsetzung der jüdischen Friedhöfe in Österreich. ${ }^{46}$

Oberstes Organ aller drei Fonds ist das Kuratorium, welches sich aus den folgenden Personen zusammensetzt:

1. Die Präsidenten des Nationalrates, 2. der Bundeskanzler, der Vizekanzler, der Bundesminister für auswärtige Angelegenheiten, der Bundesminister für Arbeit und Soziales, der Bundesminister für Finanzen und der Bundesminister für Unterricht und kulturelle Angelegenheiten (oder von diesen entsandte Vertreter aus dem jeweiligen Ressort), 3. zwölf

\footnotetext{
${ }^{45}$ https://www.ris.bka.gv.at/Dokumente/BgblPdf/1995_432_0/1995_432_0.pdf [Datum des Zugriffs: 30.8.2019]

${ }^{46}$ https://www.nationalfonds.org/organe.html [Datum des Zugriffs: 30.8.2019]
} 
weitere Mitglieder, die vom Hauptausschuss des Nationalrates gewählt werden. ${ }^{47}$

Mitglieder, die vom Hauptausschuss zu wählen sind, „[...] sind Vertreter von Opfergruppen, Mitglieder des Nationalrates und des Bundesrates sowie anerkannte Persönlichkeiten des öffentlichen, kulturellen und wissenschaftlichen Lebens in Österreich.“ (Meissner, 2005b, S.16) Zu den Aufgaben des Kuratoriums gehören die Richtlinienkompetenz, die Geschäfts- und Finanzordnung der zu erbringenden Leistungen des Fonds sowie die Beschlussfassung über das Fondsvermögen und die Kontrolle der Verwendung des Vermögens. ${ }^{48}$

Die Zusammensetzung des Komitees des Nationalfonds ist im NF-G unter Paragraph 5 geregelt.
§ 5. (Verfassungsbestimmung) (1) Dem Komitee gehören der Vorsitzende des Kuratoriums oder ein von ihm bestellter Vertreter als Vorsitzender, ein weiteres vom Kuratorium bestelltes Mitglied als Stellvertreter des Vorsitzenden sowie drei weitere Mitglieder an, die vom Vorsitzenden des Kuratoriums mit Zustimmung des Hauptausschusses des Nationalrates ernannt werden. ${ }^{49}$

Das Komitee trifft die Entscheidungen über die einzelnen Zuerkennungen der Leistungen.

Als drittes Organ wurde der Generalsekretär bestimmt, im NF-G unter Paragraph 6 geregelt. Im Falle des Nationalfonds handelt es sich seit Bestehen des Fonds um eine Generalsekretärin. Die Generalsekretärin unterstützt den/die Vorsitzende/Vorsitzenden des Kuratoriums bei der Verwaltung, bereitet die Entscheidungen und Beschlüsse vor und hat vor allem auch die Aufgabe, „die Verbindung zwischen Österreich und den im Ausland lebenden Opfern des

\footnotetext{
${ }_{48}^{47}$ https://www.nationalfonds.org/organe/kuratorium.html\#kuratorium [Datum des Zugriffs: 30.8.2019] ${ }^{48}$ ebd.

49 https://www.ris.bka.gv.at/Dokumente/BgblPdf/1995_432_0/1995_432_0.pdf [Datum des Zugriffs: 30.8.2019]
} 
Nationalsozialismus zu pflegen. “50 Wie in Paragraph 1, Absatz 3 des NF-G formuliert, besitzt der Nationalfonds eine eigene Rechtspersönlichkeit. ${ }^{51}$

Um eine möglichst rasche Unterstützung für die Opfer, angesichts des hohen Alters der Überlebenden, vornehmen zu können, erfolgte auch der Aufbau des Fonds sehr unbürokratisch. Zu Beginn wurde ein provisorisches Büro in einem Sitzungszimmer des Parlamentsgebäudes eingerichtet (vgl. Meissner, 2005b, S.20). Nach der Nominierung der Generalsekretärin wurden eigene Büroräumlichkeiten bezogen, Antragsformulare entwickelt und versucht, den Zugang der Antragstellerlnnen möglichst niederschwellig zu halten (vgl. ebd.). In der ersten Zeit seines Bestehens bestand das Team des Nationalfonds aus der Generalsekretärin, deren Stellvertreterin, einigen ReferentInnen, einem Sekretariat, einer Finanzabteilung und einer Projektbetreuung sowie aus einem Datenbankbeauftragten und einigen freien Dienstnehmerlnnen (vgl. Lessing, 2000, S.74). Bis Juli 2019 bestand der Nationalfonds aus den folgenden Abteilungen, die der Geschäftsleitung unterstanden: Archiv, Dokumentation Lebensgeschichten, Finanz, Historische Recherche, IT, Juristische Sach- und Fallbearbeitung, Koordinierungsstelle der Neugestaltung der österreichischen Länderausstellung in Auschwitz-Birkenau, Kommunikation mit Antragstellenden, Kunstrestitution, Presse und Medien, Personal, Projektförderung und Sekretariat. ${ }^{52}$

Im Juli 2019 wurde eine neue Organisationsstruktur eingeführt, die ab Jänner 2020 vollständig implementiert sein wird. Die vorliegende Arbeit untersucht unter anderem diesen Umstrukturierungsprozess empirisch.

\subsection{Die Aufgaben des Nationalfonds}

Im folgenden Kapitel werden die Hauptaufgabengebiete des Nationalfonds erläutert, die seit der Gründung des Fonds erweitert wurden. Im Vordergrund standen von Anfang an die Entschädigungszahlungen und die Projektförderungen. Weitere Aufgaben beinhalten Rechercheangebote und Informationsweitergabe an die Antragstellerlnnen, deren Nachkommen, sowie an interessierte Personen.

\footnotetext{
${ }^{50}$ https://www.ris.bka.gv.at/Dokumente/BgblPdf/1995_432_0/1995_432_0.pdf [Datum des Zugriffs: 30.8.2019]

51 ebd.

52 https://www.nationalfonds.org/team.html [Datum des Zugriffs: 30.8.2019]
} 
Die symbolische Gestezahlung und weitere Individualzahlungen

Die Hauptaufgabe des Nationalfonds besteht seit seiner Gründung in der Auszahlung der sogenannten symbolischen Gestezahlung. Diese Zahlung wurde am 17. Oktober 1995 vom Kuratorium des Nationalfonds anerkannt und stellt eine einmalige Pauschalzahlung in Höhe von 5.087,10 Euro (70.000 ATS) dar. Der Nationalfonds hat für Anträge auf die symbolische Gestezahlung keine Frist vorgesehen. Anträge können demnach noch heute gestellt werden (vgl. Meissner, 2005a, S.10).

Nicht nur die monetäre Entschädigung als finanzielle Unterstützung war und ist für die überlebenden Opfer des Nationalsozialismus von Bedeutung. Für viele Antragstellerlnnen sind vor allem die damit verbundene Anerkennung des offiziellen Österreich und die Wahrnehmung der individuellen Verfolgungsschicksale von ungleich größerem Wert. ${ }^{53}$ Angesichts der sich verändernden wissenschaftlichen Erkenntnisse wurden die Opfergruppen, die zur Antragstellung beim Nationalfonds berechtigt sind, gemäß den im NF-G festgelegten Verfolgungsgründen laufend erweitert. So wurden Menschen, denen von den Nationalsozialisten ,Asozialität vorgeworfen wurde sowie Menschen, die aufgrund ihrer sexuellen Orientierung verfolgt wurden, erstmals vom Nationalfonds als Opfer des Nationalsozialismus anerkannt. Bis zur Gründung des Nationalfonds im Jahr 1995 blieb diesen Menschen eine Anerkennung verwehrt. Weitere Opfergruppen, die vom Nationalfonds anerkannt wurden, waren unter anderem Personen mit einem jüdischen Elternteil, Kärntner PartisanInnen, Hinterbliebene von in Haft oder in Konzentrationslagern verstorbene Personen sowie die sogenannten ,Spiegelgrund - Kinder' [Kinder, die in der Jugendfürsorgeanstalt ,Am Spiegelgrund' in Wien festgehalten wurden und medizinischen Experimenten der Nationalsozialisten ausgesetzt waren, Anm. d. Verf.]. ${ }^{54}$

Die sukzessive Erweiterung des Opferbegriffes sollte, wie im NF-G festgelegt, zur „[...] Erbringung von Leistungen an alle aus Österreich stammenden Opfer des Nationalsozialismus [...]“ (Meissner, 2015, S.46) beitragen.

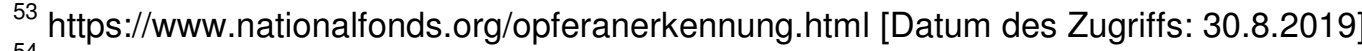

${ }^{54}$ ebd.
} 
Zu den monetären Leistungen des Nationalfonds zählt nicht nur die einmalige Gestezahlung, sondern auch weitere Zahlungen. „Für sozial bedürftige Personen besteht in Einzelfällen die Möglichkeit einer Auszahlung bis zur dreifachen Höhe des Grundbetrages. ${ }^{\text {“55 }}$ Personen, die die Voraussetzungen der Gestezahlung nicht gänzlich erfüllen, haben die Möglichkeit, andere Individualzahlungen zu erhalten:

- Zahlung aus dem Härteausgleichsfonds

- Zahlung aus dem Raubgoldfonds

- Zahlung aus Kunstverwertungserlösen

- Mietrechtsentschädigung ${ }^{56}$

\section{Härteausgleichsfonds}

Der Härteausgleichsfonds wurde im Jahr 1999 gegründet und beim Nationalfonds eingerichtet. Er sollte Zahlungen an Personen ermöglichen, die zwar Opfer der Verfolgung durch die Nationalsozialisten waren, die Voraussetzungen für eine Gestezahlung jedoch nicht erfüllten, für die eine Ablehnung aber dennoch eine „[...] besondere Härte $[\ldots]^{u 57}$ bedeuten würde. Das Budget des Härteausgleichsfonds wurde aus Projektmitteln finanziert und war mit 508.710 Euro (7 Millionen ATS) dotiert. $^{58}$

\section{$\underline{\text { Raubgoldfonds }}$}

Der Raubgoldfonds wurde seit dem Jahr 1998 vom Nationalfonds verwaltet und existierte bis zum Jahr 2010, als die Mittel aufgebraucht waren. Die Gelder für den Raubgoldfonds kamen aus dem ,Nazi Persecutee Relief Fund“. „Dieser speiste sich aus Mitteln, die aufgrund des Verzichts diverser Staaten auf inren Restbestand am so genannten Raubgold frei geworden waren. Der Nationalfonds verwaltete den auf

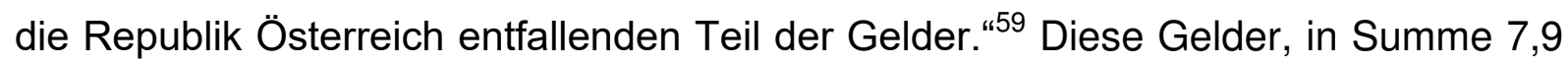

\footnotetext{
${ }^{55} \mathrm{https}: / / \mathrm{www}$. nationalfonds.org/individualzahlungen-des-nationalfonds.html [Datum des Zugriffs: 30.8.2019]

56 vgl. ebd.

57 ebd.

58 vgl. ebd.

59 ebd.
} 
Millionen Euro (109 Millionen ATS) dienten der Projektförderung und weiteren Individualzahlungen (vgl. Meissner, 2005a, S.53).

\section{Zahlungen aus Kunstverwertungserlösen}

Im Jahr 1998 wurde das Bundesgesetz über die Rückgabe von Kunstgegenständen und sonstigem beweglichem Kulturgut aus den österreichischen Bundesmuseen und Sammlungen und aus dem sonstigen Bundeseigentum, kurz Kunstrückgabegesetz (KRG) genannt, beschlossen, welches den Nationalfonds mit der Aufgabe betraute, Kunstgegenstände, deren Erben nicht mehr festgestellt werden können, zugunsten der Opfer des Nationalsozialismus zu verwerten. ${ }^{60} \mathrm{Um}$ sicherstellen zu können, dass nicht doch vielleicht noch rückstellungsberechtigte Personen existieren, hat der Nationalfonds im Jahr 2006 die Online-Kunstdatenbank erstellt, die seitdem laufend erweitert wird und derzeit ca. 9000 Kunstobjekte umfasst. Mit dieser Datenbank wird Personen ermöglicht, gezielt nach entzogenen Kunstgegenständen zu suchen. ${ }^{61}$ Sollten sich Objekte tatsächlich als erblos herausstellen, so werden diese vom Nationalfonds verwertet und der Erlös kommt Opfern des Nationalsozialismus zugute, die aufgrund fehlender Anspruchsberechtigungen keine Gestezahlung vom Nationalfonds erhalten haben. ${ }^{62}$

\section{Mietrechtsentschädigung}

„In Umsetzung des Washingtoner Abkommens wurde der Nationalfonds der Republik Österreich für Opfer des Nationalsozialismus im Jahr 2001 durch Gesetzesnovelle (BGBI. I Nr. 11/2001) mit der Entschädigung für entzogene Mietrechte, Hausrat und persönliche Wertgegenstände betraut. “63 In der Gesetzesnovelle wurde der neue Paragraph $2 \mathrm{~b}$ erfasst. Für die Mietrechtsentschädigung wurden 150 Millionen USDollar bereitgestellt. Die Antragsfrist lief bis zum 30. Juni 2004. In dieser Zeit wurden an insgesamt über 20.000 Antragstellerlnnen bzw. deren Erblnnen die Pauschalsumme von 7.630 Euro (7.000 US-Dollar) bzw. eine Nachzahlung in Höhe

\footnotetext{
${ }^{60} \mathrm{https}: / /$ www.ris.bka.gv.at/GeltendeFassung.wxe?Abfrage=Bundesnormen\&Gesetzesnummer $=10010$ 094 [Datum des Zugriffs: 30.8.2019]

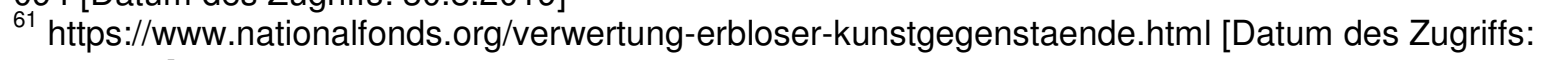

30.8.2019]

${ }^{62} \mathrm{vgl}$. ebd.

${ }^{63}$ https://www.nationalfonds.org/mietrechtsentschaedigung.html [Datum des Zugriffs: 30.8 .2019 ]
} 
von je 1.000 Euro ausbezahlt. Die restliche Summe, die nicht mehr ausbezahlt werden konnte, kommt auf Grundlage einer Gesetzesnovelle des Nationalfondsgesetzes im Jahr 2013 den Opfern des Nationalsozialismus in Form von Programmen zugute. ${ }^{64}$

\section{Projektförderung}

Ein weiteres Aufgabengebiet des Nationalfonds ist die Projektförderung, welche seit dem Jahr 1996 besteht. Prioritär wurden und werden derzeit noch Projekte gefördert, welche die noch lebenden Opfer des Nationalsozialismus unterstützen, „[...] insbesondere soziale, medizinische sowie psychotherapeutische Projekte [...].“65

Darüber hinaus werden aber auch Projekte gefördert, die der wissenschaftlichen Erforschung des Nationalsozialismus und des Schicksals seiner Opfer dienen, an das nationalsozialistische Unrecht erinnern oder das Andenken an die Opfer wahren. Bildungspolitischen Projekten und Gedenkprojekten kommt ein besonders hoher Stellenwert zu. ${ }^{66}$

Die Finanzierung der Projekte erfolgt durch das Budget des Nationalfonds. Insgesamt wurden bisher rund 2000 Projekte in Höhe von über 30 Millionen Euro gefördert. ${ }^{67}$

\section{Lebensgeschichten}

Auf der Website des Nationalfonds wird auf die Bedeutung der Publikation von lebensgeschichtlichen Erinnerungen der Opfer des Nationalsozialismus eingegangen:

\footnotetext{
${ }^{64}$ https://www.nationalfonds.org/mietrechtsentschaedigung.html [Datum des Zugriffs: 30.8 .2019$]$

${ }^{65} \mathrm{https}: / / \mathrm{www}$.nationalfonds.org/projektfoerderung.html [Datum des Zugriffs: 30.8 .2019 ]

${ }^{66} \mathrm{ebd}$.

${ }^{67}$ vgl.ebd.
} 
Der Nationalfonds der Republik Österreich für Opfer des Nationalsozialismus erachtet die Aufarbeitung, Dokumentation, Veröffentlichung und Zugänglichmachung der Lebensgeschichten von Opfern des Nationalsozialismus als äußerst wichtig für die Aufarbeitung der jüngeren österreichischen Geschichte. Autobiografische Aufzeichnungen von Überlebenden des Holocaust dienen der Geschichtsforschung als wichtige Quelle und leisten einen wesentlichen Beitrag zur kritischen Bewusstseinsbildung kommender Generationen. Über die authentischen Lebensberichte von Betroffenen wird überdies eine sehr persönliche Dimension von Geschichte spürbar. ${ }^{68}$

Im Jahr 2000, anlässlich des fünfjährigen Bestehens, veröffentlichte der Nationalfonds die ersten Lebensgeschichten in dem Band ,In die Tiefe geblickt'. 2008 wurden erstmals lebensgeschichtliche Erinnerungen auf der Website des Nationalfonds veröffentlicht. „Diese Online-Sammlung der Lebensgeschichten wird seitdem laufend erweitert [...].699 Einzelne Lebensgeschichten wurden in den verschiedenen Jubiläumsbänden des Nationalfonds veröffentlicht. Seit dem Jahr 2011 wird die Buchreihe ,Erinnerungen. Lebensgeschichten von Opfern des Nationalsozialismus' vom Nationalfonds herausgegeben. Die Bände beinhalten Lebensgeschichten der unterschiedlichen Opfergruppen und werden österreichischen Schulen und Schulbibliotheken kostenlos angeboten. „Die persönlichen Erinnerungen und Erzählungen vieler tausender Menschen, die diese im Zuge ihrer Antragstellung beim Nationalfonds hinterlassen haben, stellen in diesem Sinne einen wertvollen geschichts- und gesellschaftspolitischen ,Schatz' dar. ${ }^{70}$

\footnotetext{
${ }^{68} \mathrm{https}: / / w w w . n a t i o n a l f o n d s . o r g / l e b e n s g e s c h i c h t e n . h t m l$ [Datum des Zugriffs: 30.8 .2019$]$

${ }^{69} \mathrm{ebd}$.

70 ebd.
} 
Neugestaltung der österreichischen Länderausstellung in Auschwitz-Birkenau

Der Nationalfonds wurde im Jahr 2009 von der Bundesregierung mit der Koordinierung, Planung und Abwicklung der Neugestaltung der österreichischen Länderausstellung im ehemaligen Konzentrations- und Vernichtungslager AuschwitzBirkenau beauftragt. ${ }^{71}$

Die Länderausstellungen dokumentieren das Schicksal der Menschen, die aus den jeweiligen Ländern nach Auschwitz-Birkenau deportiert wurden. Die Länderausstellung der Republik Österreich wurde am 19. März 1978 eröffnet und war ein Kind inrer Zeit. In der Ausstellung wurde Österreich noch als das erste Opfer des Nationalsozialismus deklariert. Anfang der 2000er Jahre fand aufgrund der aufkommenden Kritik an der Ausstellung eine wissenschaftliche Auseinandersetzung statt. Es wurden Untersuchungen und Analysen durchgeführt und im Jahr 2008 in einem Bericht, der vom Nationalfonds gefördert wurde, veröffentlicht. Die gewonnenen Erkenntnisse flossen in die Neugestaltung mit ein. Die alte Ausstellung wurde umfassend dokumentiert und im Jahr 2013 im Einverständnis mit dem Museum Auschwitz-Birkenau abmontiert und in Österreich archiviert. Im Jahr 2015 wurde vom Nationalfonds ein Dokumentationsband über die Ausstellung von 1978 veröffentlicht. $^{72}$

Der Nationalfonds ist als Auftraggeber für die Realisierung der gesamten Neugestaltungsprozesse verantwortlich und trifft Entscheidungen in Absprache mit dem Steering Committee des Projekts. Neben Begleitprojekten wie z.B. der Erstellung einer eigenen Ausstellungswebsite und eines Buchbandes zur bisherigen Ausstellung stehen die Betreuung der Ausstellungsentwicklung sowie die dafür erforderliche Sanierung des ehemaligen Häftlingsblocks 17, in dem die Ausstellung untergebracht sein wird, im Vordergrund. ${ }^{73}$

\footnotetext{
${ }^{71} \mathrm{https}: / /$ www.nationalfonds.org/projektinfo.html [Datum des Zugriffs: 30.8 .2019 ]

${ }^{72} \mathrm{https}$ ://www.nationalfonds.org/ausstellung-1978.html [Datum des Zugriffs: 30.8.2019]

${ }^{73} \mathrm{https}$ ://www.nationalfonds.org/projektinfo.html [Datum des Zugriffs: 30.8.2019]
} 
Neben dem Steering Committee wird das Projekt von einem Gesellschaftlichen Beirat und einem Wissenschaftlichen Beirat unterstützt, um die wissenschaftliche Qualität zu garantieren. ${ }^{74}$

Das Projekt der Neugestaltung wurde europaweit ausgeschrieben und im Jahr 2014 wurde die Arbeit an der inhaltlichen Gestaltung von einem Team aus Kuratorlnnen und WissenschaftlerInnen unter der Leitung von Mag. Hannes Sulzenbacher begonnen. ${ }^{75}$

Wie bereits die Ausstellung von 1978, wird sich auch die neue Länderausstellung im Block 17 des Stammlagers Auschwitz befinden. Im Zuge der Neugestaltung der Ausstellung wurde der Nationalfonds im Jahr 2011 auch mit der Sanierung des Gebäudes, welches, wie das gesamte ehemalige Konzentrations- und Vernichtungslager Auschwitz-Birkenau seit 1979 unter Denkmalschutz steht und UNESCO Welterbe ist, beauftragt. Österreich unterstützt den Erhalt der Gedenkstätte mit einem Betrag von 6 Millionen Euro. Dieser Betrag dient der Dotierung der Stiftung Auschwitz-Birkenau und auch der Sanierung des Blocks 17. Wann die Sanierung abgeschlossen sein wird, kann derzeit nicht gesagt werden, da etliche Auflagen, Fristen, sowie Abstimmungen mit dem Museum Auschwitz-Birkenau einzuhalten sind. ${ }^{76}$

\section{Findbuch}

Im Zuge der Antragserfassung und -bearbeitung im Allgemeinen Entschädigungsfonds mithilfe von historischen Dokumenten wurden durch Unterstützung österreichischer Archive zahlreiche Daten zusammengetragen und von MitarbeiterInnen des Allgemeinen Entschädigungsfonds bearbeitet, ergänzt und in Datenbanken zusammengefasst. Aus diesen Datenbanken entstand die Grundlage des ,Findbuch für Opfer des Nationalsozialismus‘. ${ }^{77}$

\footnotetext{
${ }_{74}^{74}$ https://www.nationalfonds.org/projektinfo.html [Datum des Zugriffs: 30.8 .2019$]$

75 vgl. ebd.

${ }^{76}$ https://www.nationalfonds.org/sanierung.html [Datum des Zugriffs: 30.8 .2019$]$

77 https://www.findbuch.at/ueber-uns.html [Datum des Zugriffs: 30.8.2019]
} 
Dieses Online-Findbuch soll den NS-Opfern und ihren Nachkommen, Historikerinnen und Historikern oder Mitgliedern lokaler Gedenkinitiativen sowie einer interessierten Öffentlichkeit den Zugang zu und die Suche in österreichischen Archivbeständen erleichtern und somit die familiengeschichtliche, wissenschaftliche, bildungspolitische und zivilgesellschaftliche Auseinandersetzung mit dem Nationalsozialismus und seinen Nachwirkungen in Österreich fördern und unterstützen. ${ }^{78}$

Das Findbuch bietet eine umfassende Sammlung an Informationen zu verschiedenen Themenbereichen: Es können damit beispielsweise Akten zu Vermögensentziehungen der NS-Zeit, Restitutionsmaßnahmen in Österreich in der Nachkriegszeit, Arisierungsakten, Vermögensanmeldungen sowie Akten der Rückstellungskommissionen gefunden werden. Das Findbuch bietet eine archivübergreifende Suchmöglichkeit nach Personen, Unternehmen und Adressen. Es besteht derzeit aus 202.115 Datensätzen, die laufend erweitert werden.

Dadurch stellt es eine der umfangreichsten Sammlungen von Informationen über die Vermögensentziehungen während der NS-Zeit sowie Restitutionsund Entschädigungsmaßnahmen nach 1945 auf dem Gebiet der Republik Österreich dar. Zudem bietet das Findbuch eine Suche in digitalisierten historischen Adressbüchern und Amtskalendern, wodurch Recherchen zu Wohnadressen und Firmensitzen auch ohne Registrierung ermöglicht werden. $^{79}$

\footnotetext{
${ }^{78}$ https://www.findbuch.at/ueber-uns.html [Datum des Zugriffs: 30.8.2019]

${ }^{79}$ https://www.findbuch.at/das-findbuch.html [Datum des Zugriffs: 30.8.2019]
} 


\subsection{Allgemeiner Entschädigungsfonds für Opfer des Nationalsozialismus}

Der Allgemeine Entschädigungsfonds für Opfer des Nationalsozialismus [im Folgenden Allgemeiner Entschädigungsfonds genannt, Anm. d. Verf.] wurde 2001 infolge des Washingtoner Abkommens (ein Abkommen zwischen der Österreichischen Bundesregierung und der Regierung der Vereinigten Staaten von Amerika zur Regelung von Fragen der Entschädigung und Restitution für Opfer des Nationalsozialismus) ${ }^{80}$ eingerichtet und besitzt eine eigene Rechtspersönlichkeit. ${ }^{81}$ Die Rechtsgrundlage bildet das Bundesgesetz über die Einrichtung eines Allgemeinen Entschädigungsfonds für Opfer des Nationalsozialismus und über Restitutionsmaßnahmen (Entschädigungsfondsgesetz) idgF (StF:BGBI. I Nr.12/2001). Seine Organe sind, wie beim Nationalfonds, das Kuratorium und die Generalsekretärin, sowie ein unabhängiges Antragskomitee (siehe auch Abb.1, S.49).

Der Allgemeine Entschädigungsfonds hatte die Aufgabe, Vermögensverluste zu entschädigen, welche bis dahin entweder gar nicht oder nur unzureichend entschädigt wurden. Es galt, eine umfassende Lösung für die noch offenen Fragen der Entschädigung zu finden, um der moralischen Verantwortung Österreichs gerecht zu werden. ${ }^{82}$ Insgesamt wurden beim Allgemeinen Entschädigungsfonds 20.702 Anträge bis zum Ende der Antragsfrist am 28. Mai 2003 gestellt. ${ }^{83}$

Der Entschädigungsfonds entschädigte in insgesamt zehn verschiedenen Verlustkategorien:

- Liquidierte Betriebe einschließlich Konzessionen und anderes Betriebsvermögen

\footnotetext{
${ }^{80} \mathrm{https}: / /$ www.entschaedigungsfonds.org/gesetze.html [Datum des Zugriffs: 30.8.2019]

${ }^{81} \mathrm{https}: / / w w w . e n t s c h a e d i g u n g s f o n d s . o r g /$ ueber-uns.html [Datum des Zugriffs: 30.8.2019]

82 https://www.entschaedigungsfonds.org/vermoegensentschaedigung.html [Datum des Zugriffs: 30.8.2019]

${ }^{83}$ vgl. ebd.
} 
- Immobilien, soweit für diese nicht Naturalrestitution gemäß Teil 2 des Entschädigungsfondsgesetzes geleistet wurde

- Bankkonten

- Aktien

- Schuldverschreibungen

- Hypotheken

- Bewegliches Vermögen, soweit derartige Vermögensverluste nicht bereits durch Leistungen des Nationalfonds abgegolten wurden

- Versicherungspolizzen

- Berufs- und ausbildungsbezogene Verluste

- Sonstige Verluste und Schäden ${ }^{84}$

Dem Fonds standen 210 Millionen US-Dollar für die Auszahlungen zur Verfügung. Durch eine Gesetzesnovelle im Jahr 2009 wurde ein zusätzlicher Betrag von 4,1 Millionen US-Dollar vom Bund bereitgestellt. ${ }^{85}$ Es erfolgte eine pro rata Auszahlung, da die Höhe der Gesamtverluste die verfügbare Summe für die Entschädigungen überstieg. ${ }^{86}$ Die Antragsvoraussetzungen glichen denen des Nationalfonds:

Antragsberechtigt waren Personen oder Vereinigungen, die vom NS-Regime aus politischen Gründen, aus Gründen der Abstammung, Religion, Nationalität, sexuellen Orientierung, aufgrund körperlicher oder geistiger Behinderung oder aufgrund des Vorwurfs der so genannten Asozialität verfolgt wurden, sowie Personen, die das Land verlassen haben, um einer solchen Verfolgung zu entgehen. Weitere Voraussetzung war, dass diese Personen als Folge von oder im Zusammenhang mit Ereignissen auf dem Gebiet der

\footnotetext{
${ }^{84} \mathrm{https}: / /$ www.entschaedigungsfonds.org/vermoegensentschaedigung.html [Datum des Zugriffs: 30.8.2019]

${ }^{85}$ https://www.entschaedigungsfonds.org/meldung/rund-215-millionen-us-dollar-an-rund-25-000berechtigte-ausbezahlt.html [Datum des Zugriffs: 30.8 .2019$]$

${ }^{86} \mathrm{https}: / / \mathrm{www}$.entschaedigungsfonds.org/verfahren-vor-dem-antragskomitee.html [Datum des Zugriffs: 30.8.2019]
} 
heutigen Republik Österreich während der NS-Zeit Verluste oder Schäden erlitten haben. Antragsberechtigt waren überdies Erbinnen und Erben von antragsberechtigten Personen sowie Rechtsnachfolgerlnnen von Vereinigungen, die durch das NS-Regime aufgelöst worden waren. ${ }^{87}$

Insgesamt wurden vom Allgemeinen Entschädigungsfonds bis Anfang Mai 2019, den Verjährungsfristen entsprechend, 214,9 Millionen US-Dollar an die Anspruchsberechtigen ausbezahlt. ${ }^{88}$

Die Schiedsinstanz, welche Anträge auf Naturalrestitution von öffentlichem Vermögen prüft, ist ebenfalls beim Allgemeinen Entschädigungsfonds eingerichtet. ${ }^{89}$ Die Schiedsinstanz für Naturalrestitution besteht aus einem Gremium von drei Personen, welche „[...] die Rückstellung von in der NS-Zeit entzogenen Liegenschaften und Superädifikaten bzw. von beweglichem Vermögen jüdischer Gemeinschaftsorganisationen empfehlen, soweit diese Vermögenswerte am 17. Jänner $2001 \mathrm{im}$ öffentlichen Eigentum standen. ${ }^{90}$ Die Antragsfrist endete am 31. Dezember 2011. ${ }^{91}$ Insgesamt wurden bei der Schiedsinstanz 2.307 Anträge eingebracht, von denen 140 Anträge für die Rückstellung empfohlen wurden. „Alle Empfehlungen der Schiedsinstanz wurden von den jeweiligen Gebietskörperschaften vollständig umgesetzt. “92

Die Umsetzung der Entscheidungen, die auf eine Rückgabeempfehlung lauten, fällt in die Kompetenz des öffentlichen Eigentümers bzw. der öffentlichen Eigentümerin. Wenn die Naturalrestitution nicht zweckmäßig oder durchführbar ist (wie etwa bei öffentlichen Straßengrundstücken, Schulen oder Gemeindebauten), empfiehlt die Schiedsinstanz, einen vergleichbaren

\footnotetext{
${ }^{87}$ https://www.entschaedigungsfonds.org/vermoegensentschaedigung.html [Datum des Zugriffs: 30.8.2019]

${ }^{88}$ https://www.entschaedigungsfonds.org/meldung/rund-215-millionen-us-dollar-an-rund-25-000berechtigte-ausbezahlt.html [Datum des Zugriffs: 30.8.2019]

${ }^{89} \mathrm{https}: / /$ www.entschaedigungsfonds.org/organe.html [Datum des Zugriffs: 30.8.2019]

${ }^{90} \mathrm{https}: / / \mathrm{www}$.entschaedigungsfonds.org/naturalrestitution.html [Datum des Zugriffs: 30.8 .2019$]$

${ }^{91}$ vgl. ebd.

92 https://www.entschaedigungsfonds.org/meldung/schiedsinstanz-fuer-naturalrestitutionantragsbearbeitung-abgeschlossen.html [Datum des Zugriffs: 30.8.2019]
} 
Vermögenswert an die AntragstellerInnen zurückzustellen. In der Regel handelt es sich dabei um den Verkehrswert der Liegenschaft, der von der Schiedsinstanz auf Grundlage eines unabhängigen Sachverständigengutachtens festgestellt wird. ${ }^{93}$

Seit dem 30. November 2018 ist die Antragsbearbeitung abgeschlossen. Bis Ende August 2020 besteht jedoch noch die Möglichkeit, einen Antrag auf Wiederaufnahme des Verfahrens zu stellen. Der Schlussbericht der Schiedsinstanz für Naturalrestitution, der 2018 vorgelegt wurde, wurde am 17. Juni 2019 vom Kuratorium des Allgemeinen Entschädigungsfonds zur Kenntnis genommen. Das Kuratorium wird den Schlussbericht an den Hauptausschuss des Nationalrats übermitteln. Sobald der Hauptausschuss den Schlussbericht zur Kenntnis genommen hat, wird die Schiedsinstanz aufgelöst. ${ }^{94}$

Der Schlussbericht des Antragskomitees des Allgemeinen Entschädigungsfonds wurde am 4. April 2017 durch den Hauptausschuss des Nationalrats zur Kenntnis genommen (vgl. Nationalfonds der Republik Österreich für Opfer des Nationalsozialismus, 2019, S.27). Der Schlussbericht wird in Deutsch und Englisch auch in Buchform publiziert werden. Bis zur Auflösung der Schiedsinstanz wird der Allgemeine Entschädigungsfonds seine Funktion als Geschäftsapparat beibehalten. $\mathrm{Zu}$ den noch offenen Aufgaben zählen die Sicherung und Dokumentation der Materialen im Archiv und der Datenbanken (vgl. Nationalfonds der Republik Österreich für Opfer des Nationalsozialismus, 2019, S.25). Mit April 2019 endete die letzte Verjährungsfrist des Allgemeinen Entschädigungsfonds. „Derzeit laufen Vorkehrungen für den administrativen Abschluss des Entschädigungsfonds. Dazu zählen die Beendigung der vom Entschädigungsfonds abgeschlossenen Verträge und die Abschlussrechnung über die Mittel des Fonds. Mit der vollständigen Erfüllung seiner Aufgaben gilt der Entschädigungsfonds als aufgelöst. “95

\footnotetext{
${ }^{93}$ https://www.entschaedigungsfonds.org/verfahren-vor-der-schiedsinstanz.html [Datum des Zugriffs: 30.8.2019]

${ }_{94}$ https://www.entschaedigungsfonds.org/meldung/newsletter-juni-2019.html [Datum des Zugriffs: 30.8.2019]

${ }_{95}$ https://www.entschaedigungsfonds.org/meldung/rund-215-millionen-us-dollar-an-rund-25-000berechtigte-ausbezahlt.html [Datum des Zugriffs: 30.8.2019]
} 


\subsection{Fonds zur Instandsetzung der jüdischen Friedhöfe in Österreich}

Der Fonds zur Instandsetzung der jüdischen Friedhöfe in Österreich [in weiterer Folge Friedhofsfonds genannt, Anm. d. Verf.] wurde im Dezember 2010 für 20 Jahre beim Nationalfonds eingerichtet. Die Aufgabe des Fonds ist die Erhaltung und Restaurierung jüdischer Friedhöfe in Österreich. Die Verpflichtung der Sanierung und Erhaltung der jüdischen Friedhöfe wurde im Washingtoner Abkommen von 2001 beschlossen. ${ }^{96}$ Der Friedhofsfonds ist gleich dem Allgemeinen Entschädigungsfonds durch die gemeinsamen Organe des Kuratoriums und der Generalsekretärin eng mit dem Nationalfonds verbunden (siehe auch Abb.1, S.49). Des Weiteren begleitet ein eigens eingerichteter Beirat die Projekte und berät das Kuratorium hinsichtlich der Zuerkennung der Leistungen. ${ }^{97}$ Der Friedhofsfonds erhält vom Bund jährlich eine Million Euro, gleichzeitig müssen von den jeweiligen EigentümerInnen der Friedhöfe Beträge in gleicher Höhe bereitgestellt werden. ${ }^{98}$ Die gesetzliche Grundlage des Friedhofsfonds ist das Bundesgesetz über die Einrichtung des Fonds zur Instandsetzung der jüdischen Friedhöfe in Österreich idgF (StF: BGBI. I Nr. 99/2010). ${ }^{99}$ Die Anträge zur Instandsetzung können von den EigentümerInnen der Friedhöfe beim Fonds eingebracht werden. Eine Förderung aus dem Friedhofsfonds erfolgt unter der Prämisse, dass nach der Sanierung für die weitere Pflege und Erhaltung Sorge getragen wird, hierfür muss sich die jeweilige Standortgemeinde für mindestens 20 Jahre verpflichten. ${ }^{100}$

\subsection{Gesetzesnovelle 2017}

Wie in Kapitel 3.1. erläutert, stellt das NF-G die gesetzliche Grundlage des Nationalfonds dar. Im Jahr 2017 kam es zu einer Gesetzesnovelle, in der die bereits bestehenden Aufgaben, die bis dahin noch nicht gesetzlich verankert waren. Zusätzlich wurden die zukünftigen Aufgaben des Nationalfonds detailliert erfasst.

\footnotetext{
${ }_{96}^{96}$ https://www.friedhofsfonds.org/ueber-uns.html [Datum des Zugriffs: 30.8.2019]

${ }_{97} \mathrm{https}: / /$ www.friedhofsfonds.org/organe/beirat.html\#beirat [Datum des Zugriffs: 30.8.2019]

98 https://www.friedhofsfonds.org/ueber-uns.html [Datum des Zugriffs: 30.8.2019]

${ }^{99} \mathrm{https}: / / w w w . f r i e d h o f s f o n d s . o r g / g e s e t z e . h t m l$ [Datum des Zugriffs: 30.8.2019]

100 https://www.friedhofsfonds.org/antragstellung.html [Datum des Zugriffs: 30.8.2019]
} 
Per Bundesgesetzblatt vom 18. Oktober 2017 wurde der Beschluss des Nationalrats zur Gesetzesnovelle bekannt gemacht. Die Novelle umfasst die rechtliche Verankerung der Aufgaben, die der Nationalfonds bezüglich der Neugestaltung der österreichischen Länderausstellung im ehemaligen Konzentrations- und Vernichtungslager Auschwitz-Birkenau innehat. Zudem wurden Aufgabenbereiche betreffend die Vermittlung und Verbreitung des Wissens um den Nationalsozialismus und dessen Folgen gesetzlich neu definiert und erweitert. Der ergänzte Gesetzestext lautet wie folgt:

\section{In § 2a Abs. 1 lautet Ziffer 5:}

„5. die Dotierung der Stiftung Auschwitz-Birkenau und die Sanierung des für die österreichische Ausstellung bestimmten Pavillons der Gedenkstätte Auschwitz-Birkenau (§ 2c), die Koordinierung der Neugestaltung dieser Ausstellung, die Gewährleistung ihres Betriebs sowie die Verwaltung der bisherigen Ausstellung. Der Bund leistet dem Fonds die für die Erfüllung dieser Aufgaben erforderliche administrative Unterstützung;"

\section{In § 2a Abs. 1 werden folgende Ziffern 6 und 7 eingefügt:}

„6. die Unterstützung und Beratung für Opfer des Nationalsozialismus (insbesondere Personen gemäß § 2 Abs. 1) und ihre Angehörigen;

7. die Förderung und Verbreitung von Wissen um den Nationalsozialismus, seine Folgen und das Schicksal seiner Opfer sowie die Wahrung des Andenkens an die Opfer, insbesondere durch

a) die geordnete Erfassung und Bewahrung der von Nationalfonds und Allgemeinem Entschädigungsfonds erstellten Verfahrens- und Verfolgungsdokumentation;

b) die Sammlung, wissenschaftliche Erforschung und Dokumentation von lebensgeschichtlichen Zeugnissen von Opfern des Nationalsozialismus; 
c) die Bereitstellung und Vermittlung von Informationen zu Nationalsozialismus und Entschädigungs- und Restitutionsmaßnahmen für die Öffentlichkeit und die Erleichterung des Zuganges zu den betreffenden Materialien;

d) die Beantwortung von Anfragen in Zusammenhang mit dem Nationalsozialismus und dessen Folgen in Österreich;

e) die Zusammenarbeit mit nationalen und internationalen Organisationen, Gedenk- und Forschungseinrichtungen.“101

Durch die Gesetzesnovelle erfolgte eine explizite Beauftragung des Nationalfonds zur Bewahrung des Andenkens an die Opfer des Nationalsozialismus und zur umfassenden Bekanntmachung und Förderung des Wissens um den Nationalsozialismus und dessen Folgen. Welche Auswirkungen die Novelle für die Organisationsstruktur im Nationalfonds haben wird, wird im empirischen Teil dieser Arbeit untersucht.

101 https://www.ris.bka.gv.at/Dokumente/BgblAuth/BGBLA_2017_I_143/BGBLA_2017_I_143.pdfsig [Datum des Zugriffs: 30.8.2019] 


\section{Empirie}

In diesem Kapitel wird der methodische Ansatz dieser Arbeit beschrieben. Zuerst wird das zu erforschende epistemologische Interesse erläutert bevor näher auf die Forschungsfrage eingegangen wird. Im Anschluss daran wird das Forschungsdesign konkretisiert und die angewendeten Methoden für Untersuchung, Erhebung, Analyse und Auswertung und deren Auswahl erklärt. Abschließend werden die Ergebnisse der Untersuchung dargestellt.

\subsection{Definition des Untersuchungsgegenstandes}

Seit seiner Gründung im Jahr 1995 haben sich die Aufgabengebiete des Nationalfonds stetig erweitert. Mit der letzten Gesetzesnovelle wurden die Aufgabengebiete nochmals ausgeweitet und detaillierter definiert (siehe Kapitel 3.6.). Ein Überblick der Tätigkeiten des Nationalfonds hinsichtlich Erinnerungskultur unter Bezugnahme der Gesetzesnovelle von 2017 liegt bisher nicht vor. Die Erweiterung der Aufgaben durch die Gesetzesnovelle von 2017 und die Beendigung des Allgemeinen Entschädigungsfonds für Opfer des Nationalsozialismus mit Ende 2019 führten zu einer organisatorischen Umstrukturierung, deren schrittweise Implementierung mit 1. Juli 2019 begann. Ab dem 1. Jänner 2020 gilt die neue Organisationsstruktur für alle Bereiche des Nationalfonds. Die Herausforderungen dieser Umstrukturierung fließen ebenfalls in die empirische Untersuchung mit ein.

Die Beschäftigung mit der Organisation des Nationalfonds führte zu den Folgefragen, was man allgemein unter dem Begriff der Erinnerungskultur versteht, wie sich die österreichische Erinnerungskultur entwickelt hat und was eine Organisation, die ursprünglich vorrangig gegründet wurde, um Entschädigungszahlungen an Opfer des Nationalsozialismus zu leisten, für selbige beitragen kann.

Durch die Empirie in dieser Arbeit soll erläutert werden, was der Nationalfonds aufgrund seiner Aufgabenbereiche für die Erinnerungs- und Gedenkkultur leisten kann. Dies betrifft nicht nur durch den gesetzlichen Auftrag, basierend auf dem NF-G, sondern auch den einer sozialen Verantwortung der heutigen Gesellschaft gegenüber den Überlebenden. 


\subsection{Darstellung des Forschungsdesigns}

\subsubsection{Fragestellung}

Der Beginn jedes empirischen Forschungsprozesses liegt in der Formulierung einer Forschungsfrage. Je nach Formulierung der Forschungsfrage ergibt sich die anzuwendende Erklärungsstrategie (vgl. Gläser, Laudel, 2010, S.33f).

Aufgrund des zu erforschenden Themenkreises ergibt sich folgende Fragestellung:

Was kann der Nationalfonds aufgrund der gesetzlichen Erweiterung der Aufgabenbereiche für die österreichische Erinnerungs- und Gedenkkultur leisten und welche organisatorischen Herausforderungen ergeben sich dadurch?

Die Forschungsfrage wird klar eingegrenzt, in der Arbeit wird nicht darauf eingegangen, welche Länder und welche zusätzlichen Organisationen sich in Österreich ebenfalls mit Erinnerungs- und Gedenkkultur beziehungsweise auf welche Art und Weise sie sich damit beschäftigen.

Zusätzlich zu den Erläuterungen der Begrifflichkeiten zu Erinnerungs- und Gedenkkultur, der Gründung des Nationalfonds, dessen gesetzliche Grundlagen sowie dessen organisatorische Entwicklung, werden hier auch auch nach Meinungen und Erfahrungswerte von Mitarbeiterlnnen, der Leitung des Nationalfonds und externen ExpertInnen inkludiert. Es wurden 11 leitfadengestützte ExpertInneninterviews geführt und deren Methode wird im Folgenden näher erläutert.

\subsubsection{Methodologischer Zugang}

Es folgt nun eine Beschreibung des methodologischen Zugangs und eine Erläuterung, warum für die vorliegende Forschung der qualitative Zugang gewählt wurde. ${ }^{102}$

\footnotetext{
102 In diesem Kapitel sind Textteile meiner 2013 an der FH Campus Wien vorgelegten Gruppenbachelorarbeit mit dem Titel „Sozialökonomische Betriebe als Chance auf nachhaltige Integration in den ersten Arbeitsmarkt am Beispiel von „fix und fertig““ eingeflossen. (Dömötör M., Höller H., Keglovits B, Killic J., Mayerhofer S., 2013, S.29-34)
} 
Gläser und Laudel beschreiben drei methodologische Prinzipien für die sozialwissenschaftliche Forschung. „(1) Das Prinzip der Offenheit fordert, dass der empirische Forschungsprozess offen sein muss für unerwartete Informationen." (Gläser, Laudel, 2010, S.30) Diese Informationen können nicht erfasstes theoretisches Vorwissen beinhalten oder auch bestimmten Faktoren des Untersuchungsgegenstandes widersprechen (vgl. ebd.).

„(2) Das Prinzip des theoriegeleiteten Vorgehens betont die Notwendigkeit, an vorhandenes theoretisches Wissen über den Untersuchungsgegenstand anzuschließen, da nur so auch zu diesem Wissen beigetragen werden kann." (Hopf 1995, Meinefeld 1997, Mayring 2007:45 zit. n. Gläser, Laudel, 2010, S.31)

Dieses zweite Prinzip kann nach Gläser und Laudel von spezifischen Bedingungen abhängen und steht mitunter in Konkurrenz zum Prinzip der Offenheit (vgl. ebd.).

„(3) Das Prinzip des regelgeleiteten Vorgehens fordert, dass die Wissensproduktion expliziten (intersubjektiv kommunizierbaren) Regeln folgen muss." (ebd.) Die Analyse- und Auswertungsschritte müssen detailliert beschrieben werden, um für die Wissenschaft nachvollziehbar zu sein (vgl. Gläser, Laudel, 2010, S.32).

Als zusätzliches Prinzip der Sozialwissenschaft gilt das Verständnis über den Forschungsprozess als Mittel der Forschung und „[...] konstitutive Leistung des Forschers." (ebd, S.33)

Gläser und Laudel bezeichnen die Strategie, die empirische Forschung als qualitativ identifiziert, als mechanismenorientierte Strategie. „Diese Strategie sucht nach den Kausalmechanismen, die unter bestimmten Bedingungen bestimmte Effekte hervorbringen. Das schließt natürlich die Identifizierung von Ursachen und Wirkungen ein." (Gläser, Laudel, 2010, S.26) An die Identifizierung von Kausalmechanismen schließt dann deren Verallgemeinerung an (vgl. ebd., S.28).

Nach Gläser und Laudel gründen sich qualitative Forschungsmethoden auf der Interpretation von sozialen Sachverhalten, deren Resultat in einer mündlichen Beschreibung liegt. „Die Komplexität sozialer Sachverhalte wird nicht so sehr bei der 
Datenerhebung, sondern erst im Prozess der Auswertung schrittweise reduziert.“ (Gläser, Laudel, 2010, S.27)

Für die qualitative Forschung nach Mayring sind theoretische Grundlagen notwendig, die er mit fünf Grundsätzen, sogenannten Postulaten, beschreibt: „[...] die Forderung stärkerer Subjektbezogenheit der Forschung, die Betonung der Deskription und der Interpretation der Forschungssubjekte, die Subjekte auch in ihrer natürlichen, alläglichen Umgebung (statt im Labor) zu untersuchen, und schließlich die Auffassung von der Generalisierung der Ergebnisse als Verallgemeinerungsprozess“ (Mayring 2002, S.19).

Zur Veranschaulichung werden die von Mayring formulierten Postulate hier aufgezählt:

${ }_{2}$ Postulat 1: Gegenstand humanwissenschaftlichen Forschung sind immer Menschen, Subjekte. Die von der Forschungsfrage betroffenen Subjekte müssen Ausgangspunkt und Ziel der Untersuchung sein." (ebd., S.20)

„Postulat 2: Am Anfang einer Analyse muss eine genaue und umfassende Beschreibung (Deskription) des Gegenstandsbereiches stehen.“ (ebd., S.21)

Mayring folgt mit diesem Postulat Wilhelm Dilthey, der bereits 1894 festgehalten hat, dass ohne vorherige Deskription keine Erklärungen folgen können. Dilthey bezeichnet die Beschreibung als eine Grundlage der Geisteswissenschaften (vgl. ebd.)

„Postulat 3: Der Untersuchungsgegenstand der Humanwissenschaften liegt nie völlig offen, er muss immer auch durch Interpretationen erschlossen werden.“ (ebd., S.22)

Die Bedeutung des Untersuchungsgegenstandes wird von den verschiedenen Akteuren unterschiedlich interpretiert (vgl. ebd.). In diesem Postulat bezieht sich Mayring auf die Theorie der Hermeneutik. Diese wurde von Emerich Coreth (1969) mit vier Dimensionen näher erklärt (vgl. Coreth, 1969 zit. n. Mayring, 2015, S.30). Es sind dies die Horizontstruktur, die Zirkelstruktur, die Dialogstruktur und die Vermittlungsstruktur. Die Interpretation bezieht sich auf eine Sinnstruktur, die auf den Horizont dahinter ausgelegt wird. Für die Interpretation wird ein eigenes Vorverständnis vorausgesetzt, welches das Verständnis für den zu erforschenden 
Gegenstand öffnen soll und sich in einem Zirkel bewegt, dies wird auch als hermeneutischer Zirkel bezeichnet. Das Verständnis über das Material, welches es zu interpretieren gilt, erfolgt immer in einem Dialog zwischen dem/der Urheberln und dem/der InterpretIn. Durch die Vermittlungsstruktur zwischen dem/der InterpretIn und dem zu interpretierenden Material wird schließlich versucht, die beinhalteten Informationen zu identifizieren (vgl. Mayring, 2015, S.30).

„Postulat 4: Humanwissenschaftliche Gegenstände müssen immer möglichst in ihrem natürlichen, alltäglichen Umfeld untersucht werden." (Mayring, 2002, S.22)

Dem liegt Mayrings Annahme zugrunde, Menschen würden in einer Laborsituation anders reagieren und sich nicht natürlich verhalten (vgl. ebd.).

„Postulat 5: Die Verallgemeinerbarkeit der Ergebnisse humanwissenschaftlicher Forschung stellt sich nicht automatisch über bestimmte Verfahren her; sie muss im Einzelfall schrittweise begründet werden." (ebd., S.23)

Dies begründet Mayring damit, dass menschliches Handeln subjektiv, situativ und historisch geprägt ist. Dadurch „[...] lässt sich die Verallgemeinerung humanwissenschaftlicher Ergebnisse nicht automatisch durch ein Verfahren wie das der repräsentativen Stichprobe garantieren." (ebd.)

Die fünf Postulate von Mayring sowie die drei Prinzipien von Gläser und Laudel entsprechen den Grundzügen der vorliegenden Erhebung. Der Forschungsgegenstand bezieht sich auf Subjekte, im Konkreten auf die Führungskräfte und die MitarbeiterInnen des Nationalfonds sowie auf externe ExpertInnen, die sich beruflich und wissenschaftlich mit Erinnerungskultur in Österreich beschäftigen und aufgrund ihrer Expertisen und langjährigen Erfahrungen gezielt ausgewählt wurden. Zur Beantwortung der Forschungsfrage und für die Erkenntnis von Kausalmechanismen erschien die qualitative Methode der mündlichen Befragung am zielführendsten. Es wurden qualitative Interviews unter Verwendung eines Leitfadens geführt. In Anlehnung an Gläser und Laudel wurden theoriegeleitete Fragen in den Fragebogen aufgenommen. Im nächsten Schritt, der dem zweiten Postulat entspricht, wurden die Aussagen aus den Interviews beschrieben und anschließend interpretiert, wie es im dritten Postulat erklärt wird. Die Interpretation und Beschreibung der sozialen Sachverhalte lassen sich am 
besten durch eine mündliche Befragung erreichen. Durch die Interpretation ist eine vorurteilsfreie Forschung nur bedingt möglich, weil Interpretationen immer mit Erfahrungen, einem Vorverständnis sowie subjektiven Meinungen der Forscherlnnen zusammenhängen (vgl. Mayring 2002, S.25). Die Interviews wurden im alltäglichen Rahmen der Befragten - deren jeweiligen Büroräumlichkeiten - geführt, was im vierten Postulat als wichtig hervorgehoben wird. Nach Mayring hängt das Postulat der Subjektorientierung eng mit dem der Altagsorientierung zusammen (vgl. ebd., S.24). Auf den Forschungsgegenstand bezogen, lässt sich das fünfte Postulat insofern anwenden, als dass keine allgemein gültigen Erkenntnisse, auf quantitativer Forschung basierend, gewonnen werden sollen, sondern die subjektiven Meinungen und Einschätzungen der Befragten zur Forschungsfrage und dem Erkenntnisinteresse von Bedeutung sind. In diesem Zusammenhang wurde Mayrings Forderung nachgekommen, dem Subjekt möglichst offen gegenüberzutreten, um eine genaue Beschreibung des Forschungsgegenstandes zu erreichen (vgl. ebd., S.24f). Aus oben genannten Gründen wurde der qualitative Zugang gewählt. Durch statistische Erhebungen, wie sie im quantitativen Zugang verwendet werden, hätten die gewünschten Erkenntnisse zu den individuellen Meinungen und Erfahrungen, die im persönlichen Gespräch erfragt werden, weniger ausführlich oder gar nicht gewonnen werden können.

\subsubsection{Datenerhebung}

Als Erhebungsmethode gilt die Befragung, Interview genannt. Hier wird die Forschungsfrage auf mehrere Fragen unterteilt und so den Gesprächspartnerlnnen nähergebracht. „Die Antworten auf die Fragen sind dann die Daten, die einer Auswertung unterzogen werden." (Gläser, Laudel, 2010, S.39f)

\subsubsection{Leitfadengestütztes ExpertInneninterview}

Im Folgenden wird sowohl die Methode der leitfadengestützten Interviews als auch die Definition und Methode des ExpertInneninterviews beschrieben.

Zur Erkenntnisgewinnung wurden auf qualitativen Grundsätzen basierende Leitfadeninterviews mit den Zielgruppen geführt. Leitfadeninterviews sind nichtstandardisiert, das bedeutet, dass dem/der Interviewerln eine Liste offener 
Fragen (Leitfaden) als Basis für eine Strukturierung der Befragung dient (vgl. Gläser, Laudel 2010, S.111).

Die Interviews sollen den Eindruck eines alltäglichen Gesprächs vermitteln. Gläser und Laudel beschreiben, dass diese Methode der Befragung durch folgende Merkmale gekennzeichnet ist: „Es gelten kulturell festgelegte Kommunikationsregeln und Konventionen." (Haller 2001:129 zit. n. Gläser, Laudel, 2010, S.112) Dies bedeutet zum Beispiel, dass die Interviews auf Freiwilligkeit basieren und der/die Befragte Antworten auch verweigern kann. Ein weiteres Merkmal ist eine feste, von beiden Seiten akzeptierte Rollenverteilung zwischen den InterviewpartnerInnen. ExpertInneninterviews kennzeichnen sich dadurch, dass der/die Fragende die Leitung des Dialogs übernimmt, um auf diese Weise die zu erzielenden Informationen zu erreichen (vgl. Gläser, Laudel, 2010, S.112).

Im Zuge der Befragung steht dem/der Interviewerln auch die Möglichkeit offen, Fragen zu verändern und die Reihenfolge der Fragestellungen an den jeweiligen Gesprächsverlauf sowie an die Lebenswelt des/der Interviewpartnerln anzupassen, um, wenn notwendig, neue Fragen, die sich im Laufe des Gesprächs ergeben und für die Erkenntnisgewinnung relevant erscheinen, zu stellen (vgl. ebd.).

Als ExpertInnen im Sinne der sozialwissenschaftlichen Forschung gelten nach Gläser und Laudel Personen, die über besonderes Wissen über den zu erforschenden Sachverhalt verfügen (vgl. Gläser, Laudel, 2010, S.12). „Experte’ beschreibt die spezifische Rolle des Interviewpartners als Quelle von Spezialwissen über die zu erforschenden sozialen Sachverhalte. Experteninterviews sind eine Methode, dieses Wissen zu erschließen." (ebd.)

Für Gläser und Laudel stehen zwei Merkmale der ExpertInneninterviews im Vordergrund. So stellen ExpertInnen kein zu untersuchendes Objekt dar, sondern gelten als ZeugInnen der zu erforschenden Prozesse und des Weiteren kennzeichnen sich ExpertInnen durch ihre besondere Rolle im zu untersuchenden Kontext aus (vgl. ebd., S.12f). 
„Die Experteninterviews haben in diesen Untersuchungen die Aufgabe, dem Forscher das besondere Wissen der in die Situationen und Prozesse involvierten Menschen zugänglich zu machen." (Gläser, Laudel, 2010, S.13)

\subsubsection{Auswahl der InterviewpartnerInnen (Sampling)}

„Der Inhalt eines Interviews wird neben dem eigenen Erkenntnisinteresse vor allem durch den Interviewpartner bestimmt. Die Auswahl von Interviewpartnern entscheidet über die Art und die Qualität der Informationen, die man erhält." (Gläser und Laudel, 2010, S.117) Gläser und Laudel folgend wurden dementsprechend verschiedene Gruppen von InterviewpartnerInnen gewählt, um die relevanten Informationen zu erhalten. Es ergaben sich drei Gruppen von InterviewpartnerInnen, für jede Zielgruppe wurde je ein eigener Leitfaden entwickelt. Auf die Fragebogenkonstruktion wird nachfolgend noch näher eingegangen.

Die InterviewpartnerInnen setzen sich aus zwei internen Expertinnen des Nationalfonds, drei externen Expertinnen und sechs MitarbeiterInnen, die ebenfalls als interne ExpertInnen gelten, zusammen. Die Interviews mit 6 MitarbeiterInnen des Nationalfonds als interne ExpertInnen wurden anonym geführt.

Die beiden interviewten internen Expertinnen des Nationalfonds, die als Führungskräfte bereits selbst in verschiedenen Publikationen aufschienen und den Nationalfonds vornehmlich nach außen hin vertreten, waren einverstanden, in der vorliegenden Arbeit namentlich zitiert zu werden. Es sind dies Frau Mag. Hannah Lessing, Generalsekretärin des Nationalfonds und Frau Dr. Renate Meissner, MSc, Wissenschaftliche Leiterin und stellvertretende Generalsekretärin des Nationalfonds. Die drei externen Expertinnen, die für diese Arbeit interviewt wurden, waren ebenfalls mit der namentlichen Nennung einverstanden. Interviewt wurden Frau Dr. Susanne Janistyn-Novák, Vizedirektorin des Parlaments der Republik Österreich, Frau Ministerialrätin Mag. Martina Maschke vom Bundesministerium für Bildung, Wissenschaft und Forschung (BMBWF), Abteilung V/8 Bilaterale internationale Angelegenheiten - Bildung; Holocaust Education International und Obfrau des Vereins "_erinnern.at_", sowie Frau Priv. Doz. Mag. Dr. phil Heidemarie Uhl, 
Historikerin an der Österreichischen Akademie der Wissenschaften Wien und Lehrbeauftragte an den Universitäten Wien und Graz.

Von den namentlich genannten fünf internen und externen Expertinnen wurden für die namentliche Nennung und Zitierung in der vorliegenden Arbeit schriftliche Einverständniserklärungen eingeholt, welche auch eine Veröffentlichung der Arbeit einschließen.

Die erwähnten drei Gruppierungen der Interviewpartnerlnnen wurden aufgrund des zu generierenden Wissens gebildet. Froschauer und Lueger unterscheiden hier zwischen drei Typen von Expertisen: die systeminterne Handlungsexpertise, die feldinterne Reflexionsexpertise und die externe Expertise (vgl. Froschauer, Lueger, 2003, S.37f).

Die systeminterne Handlungsexpertise umfasst „[...] vorrangig Erfahrungswissen, das aus der Teilnahme an Aktivitäten im untersuchten System entstammt [...]." (ebd., S. 38) Gemeint ist damit vorrangig Wissen von Mitarbeiterlnnen eines Unternehmens (vgl. ebd.). Die feldinterne Reflexionsexpertise bezieht sich auf Wissen von AkteurInnen, die im Umfeld des zu erforschenden Feldes tätig sind (z.B. AußendienstmitarbeiterInnen oder Betriebsratsmitglieder) (vgl. ebd.). „Dieses Wissen entwickelt sich in erster Linie dort, wo Akteurlnnen auf die Berücksichtigung der Sichtweisen anderer Personen angewiesen sind und in ihren Interaktionen immer wieder systeminterne und - externe Grenzen überschreiten." (ebd.) Die externe Expertise charakterisiert die Interviewpartnerinnen, die „[...] über fundiertes theoretisches Wissen über den Gegenstandsbereich, den sie von verschiedenen Seiten und in verschiedensten (intra- und interdisziplinären) Facetten beleuchten kann [...]." (ebd.) Dieses Sonderwissen umfasst die Gruppe der externen ExpertInnen.

Auf die vorliegende Auswahl der Interviewpartnerlnnen bezogen, kann die Gruppe der internen ExpertInnen (MitarbeiterInnen) der systeminternen Handlungsexpertise zugerechnet werden, die Gruppe der internen Expertinnen (Führungskräfte) kann aufgrund ihrer Tätigkeiten und Funktionen sowohl der systeminternen 
Handlungsexpertise als auch der feldinternen Reflexionsexpertise zugerechnet werden. Die Gruppe der externen Expertinnen wird als externe Expertise eingestuft.

Aufgrund des variierenden Expertenwissens wurden auch die Fragen der Interviewleitfäden entsprechend adaptiert. Die großen Themenbereiche zu Nationalfonds und Erinnerungskultur beinhalteten in allen Fragebögen die gleichen Leitfragen. Bei den weiteren Interviewfragen wurden die Fragebögen an die jeweiligen Expertisen angepasst.

In den Befragungen der externen Expertinnen standen vor allem Fragen zur Veränderung der österreichischen Erinnerungskultur, der zukünftigen Vermittlung von Erinnerungskultur, dem Weiterbestehen nach der Ära der ZeitzeugInnen und die Kritik an der Erinnerungskultur im Vordergrund. Des Weiteren wurden Fragen zu Bedeutung und Leistungen des Nationalfonds für die österreichische Erinnerungskultur, in Bezug auf die Forschungsfrage, gestellt. Da eine externe Expertin maßgeblich an der Gründung des Nationalfonds beteiligt war, galten zentrale Leitfragen dieser Gründungszeit und der organisatorischen Entwicklung.

Zentrale Leitfragen in den Befragungen der internen Expertinnen (Führungskräfte) betrafen ebenfalls die Gründung des Nationalfonds und die organisatorischen Veränderungen, da beide Interviewpartnerinnen den Nationalfonds aufgebaut haben. Aufgrund des großen Expertenwissens betreffend äußere Einflussfaktoren, Kooperationen und die gesetzliche Einbettung des Nationalfonds wurde daher auch explizit nach der letzten Gesetzesnovelle und der Bedeutung des Nationalfonds für die österreichische Erinnerungskultur gefragt.

Die MitarbeiterInnen als interne ExpertInnen wurden vor allem nach ihren persönlichen Einschätzungen zur Zukunft des Nationalfonds, zu den organisatorischen Veränderungen aufgrund der letzten Novelle des Nationalfondsgesetzes und zu der österreichischen Erinnerungskultur befragt, da sämtliche InterviewpartnerInnen langjährige Mitarbeiterlnnen sind und über das nötige Erfahrungswissen verfügen. 


\subsubsection{Durchführung der Interviews}

Die InterviewpartnerInnen wurden teilweise per Email und teilweise persönlich kontaktiert und um ein Interview gebeten. Zur Vorabinformation über das Forschungsthema und die Herangehensweise wurde den externen Expertinnen (Dr. Janistyn-Novák, Mag. Maschke und Dr. Uhl) eine Zusammenfassung der wichtigsten Informationen übermittelt. Alle Personen, die um ein Interview gebeten wurden, haben dem Interview zugestimmt. Darauffolgend wurden die Interviewtermine vereinbart. Die Interviews mit den externen Expertinnen fanden jeweils in deren Büroräumlichkeiten statt, um das Alltagsumfeld zu gewährleisten. Die Interviews mit den internen ExpertInnen fanden ebenfalls in deren jeweiligen Büros, bzw. in einem, zum Zeitpunkt des Interviews verfügbaren, leeren Büro im Nationalfonds statt. Es wurde darauf geachtet, dass eine entspannte Gesprächsatmosphäre herrscht. Im Anschluss an die jeweiligen Interviews wurde ein Protokoll mit den wichtigsten Informationen über Situation, Setting und Eindrücke, sowie einer Deskription der Rahmenbedingungen angefertigt (vgl. Gläser, Laudel, 2010, S.192).

\subsubsection{Fragebogenkonstruktion}

Bei der Konstruktion der Fragebögen wurde darauf geachtet, mit Einstiegsfragen zu beginnen beziehungsweise die jeweiligen Expertisen der InterviewpartnerInnen zu berücksichtigen. Den jeweils ersten Fragen ging eine Einführung in das Gesprächsthema voraus, die Vermittlung des Forschungsinteresses sowie die mündliche Einverständniserklärung zur digitalen Aufzeichnung des Gespräches und die Mitteilung, dass das Interview anonym ist. Bei den externen Expertinnen und den Führungskräften als interne Expertinnen wurde das Einverständnis zur namentlichen Nennung eingeholt.

Der Interviewleitfaden dient als Erhebungsinstrument. Die Interviewfragen werden für die InterviewpartnerInnen so aufbereitet, dass sie ein Grundgerüst für die Befragung darstellen. Der Interviewleitfaden stellt dem/der Interviewerln die Reihenfolge der Fragen frei, um sich dem Gespräch anpassen zu können (vgl. Gläser, Laudel, 2010, S.142). Auch ein Nachfragen, wenn neue Themen zu Tage treten, wurde erlaubt (vgl. ebd. S.144). Dem Interviewleitfaden voran stehen Überlegungen zum Forschungsinteresse und der empirischen Methode (vgl. ebd. S.143). Um sämtliche 
Antworten zu erhalten, stellt der Interviewleitfaden die schriftliche Übersicht dar (vgl. Gläser, Laudel, 2010, S.143). „Er stellt sicher, dass in einer größeren Anzahl von Interviews gleichartige Informationen erhoben werden, und dass in jedem Interview alle Informationen erhoben werden, von denen man sich vorher überlegt, dass man sie braucht." (ebd.)

Die Interviewleitfäden beinhalteten für die internen ExpertInnen jeweils zwei Einstiegsfragen, bei den externen ExpertInnen wurde mit Fragen, ihre Expertisen betreffend, begonnen, um an den Alltag der Befragten anzuschließen (vgl. ebd., S.142f). Aufgrund der unterschiedlichen Expertisen der drei Gruppen der Befragten wurden daher drei Fragebögen konstruiert, wobei die Fragebögen der drei externen ExpertInnen minimal an die jeweiligen Expertisen angepasst wurden.

Bei der Fragebogenkonstruktion wurde darauf geachtet, dass Fragen thematisch und chronologisch zusammenpassend formuliert wurden. Die Fragen wurden klar und offen formuliert, es wurden jedoch auch Meinungsfragen gestellt, da anhand des Forschungsinteresses auch die Einschätzungen und Bewertungen der Interviewpartnerlnnen von Interesse waren (vgl. ebd., S.145).

Dabei wurde das Verhältnis von Leitfragen und weiteren Fragen beachtet. Die Fragebögen waren jeweils 1-2 Seiten lang und umfassten 14 bis 23 Fragen, davon jeweils 2 bis 4 Leitfragen.

Im Anschluss an die Entwicklung der Fragebögen wurden die einzelnen Fragen auf ihre Relevanz, auf ihre Dimensionen, auf die Formulierung und auf die inhaltliche Struktur kontrolliert (vgl. Ullrich, 1999, S.436-437 zit. n. Gläser, Laudel, 2010, S.149.).

\subsubsection{Datenerfassung}

Hier werden die Arbeitsschritte der Datenerhebung, der Datenerfassung und der Datensicherung beschrieben. Die Interviews wurden mit Zustimmung der befragten Personen als MP3-Dateien aufgezeichnet und im Anschluss wörtlich transkribiert (vgl. Mayring, 2002, S.89). Da das Hauptinteresse jedoch bei thematisch-inhaltlichen 
Informationen von den Befragten als ExpertInnen lag, wurden Floskeln und Dialekte aus dem Transkript entfernt und eine Übertragung in das normale Schriftdeutsch durchgeführt (vgl. Mayring, 2002, S.91). Es wurde auch darauf geachtet, dass Besonderheiten, wie nichtverbale Äußerungen oder Unterbrechungen, gekennzeichnet wurden (vgl. Gläser, Laudel, 2010, S.194). Die Interviews mit den internen ExpertInnen (MitarbeiterInnen) wurden direkt nach der Transkription anonymisiert (vgl. ebd.).

\subsubsection{Analysemethoden}

Folgende zwei Analysemethoden wurden für die Auswertung der Interviews der vorliegenden Arbeit ausgewählt und herangezogen:

\subsubsection{Zusammenfassende qualitative Inhaltsanalyse}

Nach Mayring liegt die Stärke der Methode der qualitativen Inhaltsanalyse darin, dass das Textmaterial durch eine streng methodische Weise analysiert werden kann. Dabei werden die Texte in Einzelteile zerlegt und anschließend in ein vorher entwickeltes Kategoriensystem eingefügt. Diese Kategorien werden aufgrund der Grundaussagen aus den Interviews gebildet (vgl. Mayring, 2002, S.114).

Mayring sieht die Interpretationsgrundform in der Zusammenfassung: „Ziel der Analyse ist es, das Material so zu reduzieren, dass die wesentlichen Inhalte erhalten bleiben, durch Abstraktion einen überschaubaren Korpus zu schaffen, der immer noch Abbild des Grundmaterials ist" (ebd., S.115). Textstellen, die die jeweilige Kategorie betreffen, werden den entsprechenden Kategorienlisten zugeordnet (subsumiert). Wird eine neue Textstelle gefunden, die keiner Kategorienbeschreibung entspricht, wird eine neue Kategorie gebildet (vgl. ebd., S.117). Die einzelnen Kategorien werden in einer Tabelle festgehalten, um so den Ablauf nachvollziehen zu können. Dieser Schritt der Auswertung wurde in Form einer EXCEL-Datei umgesetzt.

Nachdem das Durcharbeiten des Materials abgeschlossen ist und keine neuen Kategorien gebildet werden können, wird das gesamte Kategoriensystem nochmals überarbeitet, um zu prüfen, ob die ausgewählten Texte zur Forschungsfrage passen 
(vgl. Mayring, 2002, S.117). „Das Ergebnis der Analyse ist ein Set aus Kategorien zu einer bestimmten Thematik, dem spezifische Textstellen zugeordnet sind“ (ebd.).

Danach werden, im Sinne einer Kontextanalyse, die Texte paraphrasiert, gedeutet und in Hinblick auf die Fragestellung interpretiert. Dabei werden die Texte mit den Erkenntnissen aus der Literatur, auf die in der Arbeit Bezug genommen wird, in Kontext gesetzt. Anschließend werden die überarbeiteten und interpretierten Texte strukturiert, zusammengefasst und schließlich in die Arbeit aufgenommen (vgl. ebd., S.118f).

\subsubsection{Themenanalyse}

Die Themenanalyse nach Froschauer und Lueger ist eine Form der Gesprächsanalyse, die dazu dient, einen Überblick über die verschiedenen Texte und Kategorien zu erhalten, die Kernaussagen und zentralen Themen herauszuarbeiten und ebenfalls den Kontext ihres Auftretens zu beachten (vgl. Froschauer, Lueger, 2003, S.158).

Für die vorliegende Untersuchung stellte diese Form der Auswertung eine geeignete Methode dar, da Einstellungen und Meinungen von den gefragten Personen bzw. Personengruppen zu bestimmten Themen differenziert herausgearbeitet werden konnten. Ähnlich der Vorgehensweise bei der Inhaltsanalyse wird der Text zunächst nach relevanten Themen durchsucht und gemeinsame charakteristische Elemente im Sinne der Themenanalyse herausgearbeitet. Diese Charakteristika können nun miteinander verglichen werden, um auf diese Weise unterschiedliche Aussagen zu bestimmten Themen in einem oder mehreren Interviews sichtbar zu machen (vgl. ebd., S.158f).

Im nächsten Schritt werden die Textstellen zu den einzelnen Themen zusammenfassend dargestellt, wobei auf mögliche Argumentationszusammenhänge zu anderen Kategorien verwiesen wird. Mit dieser Methode können unterschiedliche Meinungen und Aussagen innerhalb eines oder mehrerer Gespräche zu einem Thema sichtbar gemacht und anschließend dokumentiert werden. Im letzten Schritt erfolgt eine Gesamtzusammenfassung, gleichzeitig werden die Themen zur Forschungsfrage in Bezug gesetzt (vgl. ebd., S.160ff). 


\subsubsection{Kategorienbildung}

Laut Mayring dient das Kategoriensystem als zentrales Element des Analyseverfahrens. „[...] sie ermöglichen das Nachvollziehen der Analyse für andere, die Intersubjektivität des Vorgehens." (Mayring, 2015, S.51) Die thematische Anbindung an den Forschungsgegenstand ist ein zentrales Element, welches durch drei Grundverfahren der Interpretation begründet wird: die Zusammenfassung, die Explikation und die Strukturierung. (vgl. ebd., S.52). „Diese Kategorien werden in einem Wechselverhältnis zwischen der Theorie (der Fragestellung) und dem konkreten Material entwickelt, durch Konstruktions- und Zuordnungsregeln definiert und während der Analyse überarbeitet und rücküberprüft." (Mayring, 2015, S.61)

Die Kategorienbildung erfolgte anhand des Codierverfahrens, wie es Froschauer und Lueger beschreiben. „Hier handelt es sich um eine aufwendigere Variante zur Analyse der begrifflichen Struktur von Themen und deren Zusammenhänge." (Froschauer, Lueger, 2003, S.163) Es wurden Themenkategorien gebildet, die auf zentralen Aussagen in den Textpassagen beruhen. Diese Themenkategorien wurden danach durch Subkategorien erweitert. Im Anschluss daran wurden die Themenkategorien strukturiert und mit den Subkategorien verknüpft. (vgl. ebd., S.163f).

In der Auswertung wurden sowohl die deduktive Kategoriendefinition als auch die induktive Kategoriendefinition, wie sie Mayring beschreibt, angewendet. Die deduktive Kategoriendefinition geht auf das theoretische Vorverständnis zurück, die Kategorien werden „[...] in einem Operationalisierungsprozess auf das Material hin entwickelt." (Mayring, 2015, S.85) In der induktiven Kategoriendefinition wiederum spielt das theoretische Vorverständnis keine Rolle, die Kategorien leiten sich „[...] direkt aus dem Material in einem Verallgemeinerungsprozess ab [...]" (ebd.)

Die induktive Kategorienbildung wird in der ,Grounded Theory' von Strauss und Corbin als offenes Kodieren bezeichnet. „Es strebt nach einer möglichst naturalistischen, gegenstandsnahen Abbildung des Materials ohne Verzerrungen durch Vorannahmen des Forschers, eine Erfassung des Gegenstands in der Sprache des Materials." (ebd., S.86) 
Im Zuge der verwendeten Auswertungsverfahren haben sich folgende Kategorien und Subkategorien ergeben, die für die Beantwortung der Forschungsfrage von Bedeutung sind und der Übersicht halber hier nun aufgelistet werden:

\section{Kategorie 1: Organisation, Struktur und Wandel des Nationalfonds}

Subkategorien:

- Die Gründung und der Beginn des Nationalfonds

- Organisation, Organisationskultur und Strukturwandel

- Bedeutung der Beschäftigung beim Nationalfonds für die MitarbeiterInnen

- Besonderheit und Einzigartigkeit des Nationalfonds

- Veränderungen der Aufgabengebiete

Kategorie 2: Gesetzesnovelle des Nationalfondsgesetzes 2017

Subkategorien

- Besonderheiten des Nationalfondsgesetzes

- Gründe, Formulierung und Ergebnis der Gesetzesnovelle, Chancen für den Nationalfonds in der Zukunft

Kategorie 3: Umstrukturierung des Nationalfonds 2019

Subkategorien

- Gründe, Veränderungen und Auswirkungen

- Bezug zur Gesetzesnovelle 2017

Kategorie 4: Herausforderungen für den Nationalfonds

Subkategorien

- Herausforderungen nach der Gründung des Nationalfonds

- Herausforderungen nach der Umstrukturierung 2019

\section{Kategorie 5: Zukunftsperspektiven für den Nationalfonds}

Subkategorien

- Zukünftige Aufgaben des Nationalfonds

- Chancen

- Risiken 


\section{Kategorie 6: Meilensteine in der Erinnerungskultur}

Subkategorien:

- Die Serie „Holocaust“

- Der Nationalfonds der Republik Österreich für Opfer des Nationalsozialismus

\section{Kategorie 7: Erinnerungskultur - Wandel und Funktion}

Subkategorien:

- Wandel und Veränderung der Erinnerungskultur

- Funktion von Erinnerungskultur

- Leistungen des Nationalfonds für Erinnerungskultur

- Persönlicher Bezug der InterviewpartnerInnen zu Erinnerungskultur

\section{Kategorie 8: Kritik an Erinnerungskultur}

Kategorie 9: Herausforderungen und Zukunftsperspektiven für die Erinnerungskultur

Subkategorien:

- Gefahren für den Erhalt der Erinnerungskultur

- Zukünftige Wirkungsstätten für Erinnerungskultur

- Zukünftige Aufgaben der Erinnerungskultur

In der Auswertung des Datenmaterials haben sich zwei induktive Subkategorien ergeben, dies sind einerseits die Gründung des Nationalfonds als Subkategorie in der Kategorie "Meilensteine in der Erinnerungskultur" und andererseits die zukünftigen Wirkungsstätten für Erinnerungskultur als Subkategorie in der Kategorie "Zukunftsperspektiven für Erinnerungskultur". Die restlichen Kategorien und Subkategorien ergaben sich deduktiv aus dem bereits vorhandenen Vorwissen und der theoretischen Aufarbeitung des Forschungsgegenstandes. 


\subsection{Darstellung der Erhebungsergebnisse}

Im folgenden Kapitel werden nun die Aussagen aus den Interviews, welche den bereits oben genannten Kategorien und Subkategorien zugeordnet wurden, interpretiert.

\subsubsection{Kategorie 1: Organisation, Struktur und Wandel des Nationalfonds}

In dieser Kategorie wurden organisatorische Entwicklungen, strukturelle Veränderungen und der Wandel des Nationalfonds seit seiner Gründung erfasst. Es wurden dabei fünf Subkategorien entwickelt, die die Themenbereiche nochmals eingrenzen.

Die Gründung und der Beginn des Nationalfonds

In dieser Subkategorie werden die Gründungszeit und der Beginn der Arbeit des Nationalfonds beschrieben sowie die Wahrnehmungen der befragten Personen erläutert.

Laut Dr. Janistyn-Novák führten verschiedene Ereignisse zu der Gründung des Nationalfonds, zum Beispiel die Haltung Amerikas und die Rede des damaligen Bundeskanzlers Vranitzky in Israel (vgl. Janistyn-Novák, S.2, Z.49-53). Die Diskussionen um die Entschädigungszahlungen und die entsprechend erweiterte Auseinandersetzung mit dem Thema war die Initialzündung für die Gründung des Nationalfonds (vgl. Janistyn-Novák, S.17, Z.612-616). Die Gründung des Nationalfonds erfolgte laut Dr. Uhl im Rahmen eines Maßnahmenbündels gegen die bisherigen Versäumnisse auf dem Gebiet (vgl. Uhl, S.5, Z.181-186). Bei der Beschlussfassung des Nationalfondsgesetzes war die Dimension der zu erwartenden Anträge nicht klar (vgl. Janistyn-Novák, S.3, Z.79-81). Zuerst wurde das Gesetz beschlossen, dann erst begannen die Überlegungen, wer und wie man sich mit der Thematik auseinandersetzen und den Nationalfonds organisieren könnte (vgl. Janistyn-Novák, S.4-5, Z.145-152). Der Nationalfonds hat eine eigene Rechtspersönlichkeit, ist dem Parlament zugeordnet, es war aber laut Dr. JanistynNovák dennoch eine "[...] vollkommen neue Aufgabe [...]." (Janistyn-Novák, S.5, Z.152-153) 
Der Nationalfonds sollte möglichst schnell seine Arbeit aufnehmen, damit das Thema nicht wieder in den Hintergrund rücken würde, wie es Dr. Janistyn-Novák formuliert (vgl. Janistyn-Novák, S.3, Z.77-79). Erst mit Bestellung der Generalsekretärin im September 1995 begann der organisatorische Aufbau des Nationalfonds mit der Suche nach Büroräumlichkeiten (vgl. Janistyn-Novák, S.5, Z.177-180). Der gelungene Aufbau des Nationalfonds war den engagierten MitarbeiterInnen zu verdanken (vgl. Janistyn-Novák, S.3, Z.100-103). Zu Beginn des Nationalfonds waren nur wenige Mitarbeiterlnnen beschäftigt, die fast rund um die Uhr ZeitzeugInnengespräche geführt haben (vgl. Janistyn-Novák, S.5, Z.180-184). In der ersten Zeit des Nationalfonds waren Arbeitsabläufe und Prozesse nicht strukturiert, sondern erst im Entstehen (vgl. Int.1, S.2, Z.47-48).

Die Organisationsstruktur stand zu Beginn nicht im Vordergrund, man hat den Fokus auf die tatsächlich anfallende Arbeit gelegt (vgl. Janistyn-Novák, S.7, Z.246-249). Erst im Laufe der Zeit entstand nach und nach eine Organisationsstruktur (vgl. Janistyn-Novák, S.7, Z.249-251). Der Nationalfonds wurde bewusst an das Parlament angegliedert, weil die Parlamentszugehörigkeit mehr Möglichkeiten bietet (vgl. Janistyn-Novák, S.17, Z.591-592).

Der Nationalfonds war zu Beginn als temporäre Institution gedacht, erzählt eine Mitarbeiterin. "[...] es hat geheißen, den gibt es vielleicht ein Jahr, maximal zwei Jahre, dann ist es vorbei." (Int.1, S.2, Z.42-43) Eine Organisationstruktur war auch laut ihrer Aussage nur bedingt vorhanden, Abteilungen sind erst später entstanden. Die Recherche wurde vornehmlich von freien Dienstnehmerlnnen betrieben (vgl. Int.1, S.3, Z.75-78).

Ein Mitarbeiter sieht die Anfangszeit des Nationalfonds als amateurhaft, unverbindlich und auch teilweise unprofessionell. Die Organisation war erst im Entstehen, das hätte man gemerkt (vgl. Int.2, S.2, Z.66-71). Die Gründung des Nationalfonds wurde "[...] dezent gehandhabt [...]" (Int.3, S.1, Z.27), wie es eine Mitarbeiterin beschreibt. Er war auf Zeit eingerichtet und sie vermutet, dass die geringe mediale Präsenz in Österreich eventuell von der Politik gewünscht war (vgl. Int.3, S.1, Z.26-30). 
Durch die anfängliche Annahme, der Nationalfonds würde nur zwei Jahre bestehen, wurde versucht, in dieser Zeit so viele Überlebende wie möglich zu finden, die anspruchsberechtigt waren. "Das hat dazu geführt, dass sich Mitarbeiter der ersten Stunde, ich sage, fast selbst ausgebeutet haben." (Int.3, S.4, Z.120-121) Es gab wenig öffentliche Unterstützung und Anerkennung. Die Aufarbeitung der Geschichte stand im Vordergrund, das Ausmaß der Arbeit war nicht erkennbar. Sie ist aber der Meinung, dass dies nicht weiter optimiert werden hätte können (vgl. Int.3, S.4, Z.119127). Die Arbeit und der Aufbau des Nationalfonds erfolgten planlos. "[...] es war ein kleines Büro mit Leuten, die irgendwie engagiert waren, aber [...] es gab ja überhaupt keinen Plan." (Int.6, S.1, Z.31-33)

Das Ausmaß der Arbeit wurde auch von der Politik unterschätzt. Die Mitarbeiterin ist der Meinung, dass es unverantwortlich war, "[...] so was zu machen, aufzustellen ohne einen Plan zu haben, oder eine Idee, ein Konzept [...]." (Int.6, S.2, Z.43-44) Beim Organisationsaufbau des Nationalfonds hätte man sich nicht auf Erfahrungswerte von früheren Organisationen gestützt. Expertisen von früheren Akteuren wie beispielsweise den Sammelstellen und dem Hilfsfonds wären nicht berücksichtigt worden (vgl. Int.6, S.2, Z.59-65).

Mag. Lessing war von Beginn an Generalsekretärin, es gab zwei Sachbearbeiterinnen, eine davon als stellvertretende Generalsekretärin im Nationalfonds. Aufgrund der hohen Besucherfrequenz der Antragstellerlnnen gab es kaum eine Arbeitsteilung, auch die Geschäftsleitung hat mit den Menschen gesprochen und viel telefoniert. Des Weiteren wurde ein Fragebogen für die Antragstellung entwickelt. Als Chefsekretärin wurde eine Dame eingestellt, die schon über eine langjährige Berufserfahrung verfügte (vgl. Lessing, S.2-3, Z.73-81). Mag. Lessing erinnert sich, dass die durchschnittliche tägliche Arbeitszeit im ersten Jahr des Nationalfonds oft bis Mitternacht gedauert hat. Sie erzählt, dass die Leute beeindruckt gewesen wären, weil der Nationalfonds fast rund um die Uhr erreichbar war, aufgrund der Zeitverschiebung auch für Antragstellerlnnen aus Amerika (vgl. Lessing, S.3, Z.81-84). In der Arbeitsteilung gab es keine Hierarchie. "Und jeder hat telefoniert, jeder hat angepackt, ich habe kuvertiert, es war keine Frage, dass die Generalsekretärin auch [...] die Fragebögen ins Kuvert steckt." (Lessing, S.3, Z.8889) Eine Struktur musste erst gefunden werden. Die fest angestellte Belegschaft des 
Nationalfonds bestand am Anfang aus sieben Mitarbeiterinnen (vgl. Lessing, S.3, Z.88-92).

Mit der Zeit kamen neue Aufgabenbereiche und weitere Entschädigungsmaßnahmen hinzu. Es folgte ein Umzug des Büros. Es gab auch zu diesem Zeitpunkt noch keine bewusst geplante Organisationsstruktur. "[...] wir sind eigentlich immer einer Struktur nachgerannt, weil wir haben einfach immer sofort gemacht [...]." (Lessing, S.5, Z.157158)

Dr. Meissner hatte das Einstellungsgespräch [1995, Anm. d. Verf.] bei Dr. JanistynNovák. Bereits in diesem Einstellungsgespräch wurden ihr 300 Korrespondenzen von potentiellen AntragstellerInnen in Deutsch, Englisch und Hebräisch vorgelegt (vgl. Meissner, S.2, Z.44-53). In diesem Gespräch hat sich auch herausgestellt, dass es sich nicht um eine rein administrative und verwaltungstechnische Aufgabe handeln würde, sondern, "[...] dass eigentlich zwar das Gesetz des Nationalfonds viele Jahre, fast 50 Jahre zu spät herausgegeben wurde, aber keine Vorkehrungen getroffen wurden, [...] wie der Nationalfonds zu gestalten sei, in welcher Weise die Organisation aufzubauen sei [...]." (Meissner, S.2, Z.46-49) Diese Freiheit in der Gestaltung hat Dr. Meissner motiviert, die Arbeit anzunehmen. Es herrschte massiver Zeitdruck, da "[...] das Nationalfondsgesetz ursprünglich ausschließlich für noch lebende Überlebende aller Opfergruppen geschaffen wurde [...]." (Meissner, S.2, Z.49-51)

Für Dr. Meissner war es eine Herausforderung, die Arbeit beim Nationalfonds zu beginnen. Es gab keine Organisation, keine Struktur, kein eigenes Büro (vgl. Meissner, S.2, Z.53-60). Die Arbeit mit den Überlebenden stand im Vordergrund. "[...] wenn es so viele Jahre zu spät kommt, habe ich mir gedacht, es wäre ganz, ganz wichtig, ein Büro für Menschen zu machen [...]." (Meissner, S.2, Z.57-58)

Der Nationalfonds war zu Beginn seiner Tätigkeit, in der Sommerpause des Parlaments im Jahr 1995, in einem Parlamentslokal [Sitzungszimmer im Parlament, Anm. d. Verf.] untergebracht. Die Gründung hatte sich bereits herumgesprochen und es kamen die ersten Personen in den Nationalfonds. Das Antragsformular war zu diesem Zeitpunkt noch nicht erstellt worden. Frau Dr. Meissner war von Anfang an 
bewusst, wie wichtig der persönliche Kontakt zu den AntragstellerInnen sein würde (vgl. Meissner, S.2, Z.60-66).

Einfach eine Ansprechstelle zu sein, dass die Leute nach so vielen Jahren des Schweigens und auch [...] mit schlechten Erfahrungen mit Behörden, [...] dass wir nicht so eine Behörde und so ein Amt sein wollen, sondern wirklich für die Menschen da sind und mit dieser Antragstellung und dieser kleinen symbolischen Gestezahlung, [...] für sie da zu sein und zuzuhören. (Meissner, S.2, Z.66-70)

Frau Dr. Meissner hatte Gestaltungsfreiheit. Bereits in den ersten 14 Tagen nach ihrem Arbeitsbeginn kamen Antragstellerlnnen aus den unterschiedlichen Opfergruppen, um ihre Lebensgeschichte zu erzählen (vgl. Meissner, S.3, Z.75-81). Laut Frau Dr. Meissner konnte man in der Anfangszeit des Nationalfonds - sie bezeichnet es als Pionierzeit - nicht von Organisationsstrukturen sprechen. Die Struktur, die es bereits gab, war aufgrund des Nationalfondsgesetzes und der bereits festgelegten Organe, wie dem Kuratorium und der Generalsekretärin, "[...] von oben definiert, top down [...]." (Meissner, S.4, Z.140)

Da die beiden Führungskräfte, Frau Mag. Lessing und Frau Dr. Meissner, sehr jung waren und wenig Erfahrung im Aufbau von Organisationen hatten, gingen sie laut Frau Dr. Meissner davon aus, dass innerhalb von drei Monaten eine funktionierende Organisationstruktur geschaffen werden könne (vgl. Meissner, S.5, Z.151-154). "Das war natürlich nicht so." (Meissner, S.5, Z.154) Die ersten Ansätze einer Büroorganisation und Struktur wurden von einer Kollegin implementiert (vgl. Meissner, S.5, Z.154-157). Laut Dr. Meissner bestand der Nationalfonds zu Beginn aus fünf bis acht Mitarbeiterinnen: das Sekretariat, die ersten freien Dienstnehmerinnen, zwei Referentinnen und die Geschäftsleitung, bestehend aus Mag. Lessing und Dr. Meissner. Für Dr. Meissner war diese Anfangszeit im Nationalfonds, wie bereits erwähnt, eine Pionierzeit. Es wurden, auch mit der Generalsekretärin, Ketten für den Versand und die Kuvertierung der Anträge gebildet, um den Arbeitsaufwand zu bewältigen (vgl. Meissner, S.5, Z.163-173). Hier 
spiegelt sich die Erzählung von Mag. Lessing, dass es eigentlich keine strenge Hierarchien gab, wider.

Auch laut Frau Dr. Meissner war der Nationalfonds zu Beginn nur als temporäre Einrichtung gedacht, "[...] die eben ausschließlich zur Abwicklung der symbolischen Gestezahlung [...]" (Meissner, S.9, Z.305-306) errichtet wurde. Die ersten drei im Gesetz verankerten Aufgaben des Nationalfonds waren die symbolische Gestezahlung, die Dreifachzahlung für sozial schwache Personen und die Projektförderung (vgl. Meissner, S.9, Z.306-310).

Sämtliche befragte Personen gaben an, dass die erste Zeit des Nationalfonds von Improvisation und Zeitdruck geprägt war. Es gab abgesehen von der Struktur, die das Nationalfondsgesetz vorgab, keine geplante Organisationsstruktur, ebenso war das tatsächliche Ausmaß der Arbeit unbekannt. Es ist dem Engagement der ersten Mitarbeiterinnen zu verdanken, dass der Nationalfonds zu einer funktionierenden Organisation wurde.

\section{Organisation, Organisationskultur, und Strukturwandel}

In dieser Subkategorie wird näher auf den Wandel des Nationalfonds eingegangen. Die Entwicklung der Organisationskultur und der Strukturwandel, sowie die Entwicklung von Aufgaben und die Umstrukturierung des Nationalfonds im Jahr 2019 werden anhand der Aussagen der befragten Personen beschrieben.

Der politische Konsens in der Bereitschaft und Offenheit im Umgang mit der Thematik war ein großer Erfolgsfaktor des Nationalfonds, beschreibt Frau Dr. Janistyn-Novák (vgl. Janistyn-Novák, S.7, Z.233-234). Anhand der unterschiedlichen Lebensgeschichten, die dem Nationalfonds von den AntragstellerInnen berichtet wurden, wurde schnell erkannt, dass man nicht anhand einer einheitlichen Strategie vorgehen konnte (vgl. Janistyn-Novák, S.7, Z.234-236). Die Arbeit des Nationalfonds wurde und wird bis heute von der Politik getragen (vgl. Janistyn-Novák, S.7, Z.241). Frau Dr. Janistyn-Novák berichtet, dass der Nationalfonds durch die beständige politische Unterstützung und Signalisierung nach außen mit der Zeit eine 
unbestrittene Institution wurde und zu einer Veränderung des öffentlichen Bewusstseins beigetragen hat (vgl. Janistyn-Novák, S.16, Z.583-588).

Für Frau Mag. Maschke ist der Nationalfonds „[...] einer der großen Geldgeber [...]" (Maschke, S.4, Z.115), von denen es nur wenige in Österreich gibt. Der Nationalfonds als Förderstelle für Projekte spricht zahlreiche zivilgesellschaftliche Initiativen an. Die hauptsächliche Zielgruppe des Nationalfonds sind die Überlebenden (vgl. Maschke, S.4, Z.115-120).

Durch die Erweiterung der Aufgabenbereiche im Laufe der Zeit, sowie durch die Gründung des Allgemeinen Entschädigungsfonds kam es zu einer Entwicklung von neuen Strukturen. Abteilungen wurden gebildet, die Organisationsstruktur wurde differenzierter (vgl. Int.1, S.3-4, Z.110-118). Manche Abteilungen, wie das Sekretariat, waren zuständig für beide Fonds, den Nationalfonds und den Allgemeinen Entschädigungsfonds. Eine Mitarbeiterin berichtet, dass viel Freiraum in der Gestaltung der Abteilung bestand (vgl. Int.1, S.4, Z.126-131). Die fehlende Struktur in den ersten Jahren des Nationalfonds konnte durch den Gestaltungsfreiraum der MitarbeiterInnen, sowie durch deren Engagement, Interessen und Kompetenzen ausgeglichen werden (vgl. Int.2, S.2-3, Z.71-76).

Die ersten Organisationsstrukturen entstanden im Team von Dr. Meissner bei den 2b- Auszahlungen [Mietrechtsentschädigung, Anm. d. Verf.]. "[...] es war schon die Arbeit im Vordergrund, aber es war ein Teamgefühl da und das war gut." (Int.3, S.4, Z.133-134) Erst ab den Jahren 2003, 2004 gab es effektiv Abteilungen im Allgemeinen Entschädigungsfonds, die laut einer Mitarbeiterin auf eine Mitarbeiterinnen-Initiative zurückgingen. Die Mitarbeiterlnnen waren sehr selbstorganisiert. hat Die Mitarbeiterin hat das Gefühl, dass es durch die Umstrukturierung im Jahr 2019 und die Einführung von mehr Leitung, für einige Mitarbeiterlnnen, die vorher mehr Eigenverantwortung gewöhnt waren, schwierig werden könnte, mit den neuen Strukturen umzugehen (vgl. Int.3, S.4, Z.135-147).

Durch die unklare Definierung der Dauer des Bestehens des Nationalfonds waren auch die Arbeitsverhältnisse immer nur befristet. Erst durch die Gesetzesnovelle von 2017 wurde laut einer Mitarbeiterin eine gewisse Sicherheit für das Bestehen des 
Nationalfonds geschaffen. Dies zeige sich auch bereits in der Zukunftsorientiertheit mancher Bereiche (vgl. Int.5, S.3, Z.75-79).

Einer weiteren Mitarbeiterin zufolge hätten die Außenkontakte im Laufe der Jahre besser gepflegt werden können. Es fand ihrer Meinung nach wenig bis kein Erfahrungsaustausch mit ähnlichen Organisationen in anderen europäischen Ländern wie Frankreich oder Deutschland statt (vgl. Int.6, S.4, Z.129-137).

Sie beschreibt auch die Kommunikation zwischen den einzelnen Abteilungen als unzureichend. MitarbeiterInnen würden zu wenig darüber informiert werden, welche Abteilung welche konkreten Aufgaben innehat. Die Außenkontakte der Geschäftsleitung und der Generalsekretärin würden ebenfalls zu wenig kommuniziert werden (vgl. Int.6, S.6, Z.201-209). Durch die neue Struktur werden die Aufgabenbereiche wieder überschaubarer, betont jedoch Frau Mag. Lessing. Durch diese Umstrukturierung bestehe nun auch die Möglichkeit, wieder eigenständig Projekte und Forschungsaufgaben zu entwickeln (vgl. Lessing, S.5, Z.169-176).

Ein Strukturwandel hat sich immer wieder im Laufe der Jahre abgezeichnet, auch durch die laufende Erweiterung der Arbeitsbereiche im Nationalfonds und Entschädigungsfonds, die eine Aufstockung der Belegschaft auf einen Höchststand von 160 Mitarbeiterlnnen zur Folge hatte, wie es Dr. Meissner beschreibt. Die Gesetzesnovelle von 2017 habe sich ebenfalls auf die Organisationsstruktur ausgewirkt, da die dort formulierten Tätigkeiten bereits teilweise ausgeführt wurden (vgl. Meissner, S.12, Z.418-430).

\section{Bedeutung der Beschäftigung beim Nationalfonds für die Mitarbeiter}

Diese Subkategorie hat sich aus den Aussagen der befragten Personen zu der Dauer ihrer Tätigkeit im Nationalfonds und im Allgemeinen Entschädigungsfonds und zu ihrer Einstellung zur Thematik der Arbeit ergeben. Um die Anonymität der Befragten zu wahren, werden die Dienstjahre nicht anhand von konkreten Zitaten, die Rückschlüsse auf InterviewpartnerInnen zulassen würden, genannt. 
Dieser Kategorie ist zu entnehmen, dass sämtliche befragte Personen bereits sehr lange in den Fonds tätig sind. Viele haben ihre Tätigkeit im Allgemeinen Entschädigungsfonds begonnen, als das Personal für die anfallenden Aufgaben im Allgemeinen Entschädigungsfonds aufgestockt wurde, und wurden dann in den Nationalfonds übernommen, als sich Aufgabenbereiche wieder verändert haben. Anhand der Antworten lässt sich feststellen, dass alle Befragten seit mindestens sechs Jahren beim Nationalfonds angestellt sind. Einige Arbeitnehmerlnnen waren vor ihrem Wechsel in den Nationalfonds bereits bis zu zehn weitere Jahre beim Allgemeinen Entschädigungsfonds angestellt. Aufgrund der durchgehend langen Beschäftigungsdauer bei den beiden Fonds wurde auch nach der Bedeutung der Arbeit für die ArbeitnehmerInnen gefragt.

Für eine Mitarbeiterin war es zu Beginn nur eine dringend benötigte Arbeitsstelle, im Laufe der Zeit habe ihr die Arbeit in diesem Bereich jedoch viel Freude bereitet (vgl. Int.1, S.1, Z.21-24). Für eine andere Mitarbeiterin bedeutet die Arbeit beim Nationalfonds viel. Es sei nicht nur ein Job, sondern biete ihr auch die Möglichkeit ihren Interessen und Talenten nachzugehen (vgl. Int.2, S.1-2, Z.35-40). Die Möglichkeit, Verantwortung für die Geschichte Österreichs mitzutragen, ist einer anderen Mitarbeiterin sehr wichtig (vgl. Int.3, S.1, Z.19-21). Die Verantwortungsübernahme war auch für eine weitere befragte Person ein zentraler Grund für die Arbeit. Für sie war es "[...] ein Anliegen, in diesem Bereich tätig zu sein, Verantwortung zu übernehmen, ein Zeichen zu setzen." (Int.4, S.1, Z.13-14) Auch eine andere befragte Person empfindet ihre Tätigkeit als gesellschaftspolitisch wichtig und misst der Beschäftigung beim Fonds große Bedeutung bei. Für sie ist es eine Aufgabe, die man tun müsse, da sie sonst niemand übernehme (vgl. Int.5, S.1, Z.12-17). Aus Sicht einer weiteren Mitarbeiterin ist die Anstellung beim Nationalfonds ebenfalls eine gesellschaftspolitische Aufgabe. Sie empfindet es als Chance, etwas für die österreichische Erinnerungskultur zu etablieren und diese lebendig zu halten (vgl. Int.6, S.1, Z.15-25).

Frau Mag. Lessing ist seit dem 1. September 1995 Generalsekretärin des Nationalfonds. Sie hat den Fonds von Beginn an aufgebaut (vgl. Lessing, S.1, Z.911). Für Frau Mag. Lessing ist es eine Lebensaufgabe geworden, den Nationalfonds 
zu leiten. Sie formuliert ihre Tätigkeit als "[...] die sinnvollste Art meine Arbeitswelt zu gestalten [...]." (Lessing, S.1, Z.15)

Frau Dr. Meissner arbeitet seit dem 24. Juli 1995 für den Nationalfonds. Sie wurde von Frau Dr. Janistyn-Novák zu den Vorarbeiten in die Parlamentsdirektion eingeladen (vgl. Meissner, S.1, Z.26-30). Für sie zählte die freie Gestaltungsmöglichkeit im Aufbau der Organisation zu der bedeutendsten Motivation in der Übernahme der Aufgabe (vgl. Meissner, S.2, Z.44-53).

\section{Besonderheit und Einzigartigkeit des Nationalfonds}

Diese Subkategorie entstand aufgrund der Fragestellung, was den Nationalfonds für die befragten Personen einzigartig macht. Daraus ergaben sich aus den Antworten folgende Alleinstellungsmerkmale:

Das Besondere am Nationalfonds laut Frau Dr. Janistyn-Novák war der Start. Die Rückstellungskommissionen in den 1950er Jahren hätten sich zwar bereits mit Entschädigung von Opfern des Nationalsozialismus beschäftigt, jedoch nicht tiefgehend genug, wie sich herausstellte. Durch die politischen Rahmenbedingungen in den 1990er Jahren hätte die Republik mit der Einrichtung des Nationalfonds auf die unzulänglichen früheren Entschädigungen reagiert (vgl. Janistyn-Novák, S.2, Z.42-48).

Frau Dr. Janistyn-Novák betont die Tatsache, dass der Nationalfonds zu Beginn ausschließlich weibliche Mitarbeiterinnen hatte (vgl. Janistyn-Novák, S.4, Z.128-131). Sie sieht den Nationalfonds als bemerkenswerte Leistung von Frauen, durch die sehr viel gelungen ist (vgl. Janistyn-Novák, S.4, Z.136-138).

Die Einzigartigkeit des Nationalfonds sieht eine Mitarbeiterin vor allem in der Belegschaft, die immer noch Teamzusammengehörigkeit und Engagement zeigen würde, um Menschen helfen zu können (vgl. Int.1, S.6, Z.200-203).

"[...] es ist die einzige Organisation, die im Verfassungsrang steht." (Int.2, S.7, Z.247) Durch den Verfassungsrang könne der Nationalfonds nicht durch einzelne Parteien 
abgeschafft werden, der befragte Mitarbeiter verweist hier in Zusammenhang mit dem Gründungsjahr des Nationalfonds auf die damals erstarkende FPÖ unter Dr. Jörg Haider. Andere Organisationen, wie die Sammelstellen in den 1950er Jahren oder der Versöhnungsfonds, wurden mit Erfüllung ihrer Aufgaben aufgelöst. Auch der Allgemeine Entschädigungsfonds würde mit Ende des Jahres 2019 aufgelöst werden, aber der Nationalfonds bleibe bestehen. Laut inm kann dies auch als besondere Priorisierung interpretiert werden (vgl. Int.2, S.7-8, Z.258-262).

Eine weitere Mitarbeiterin sieht das Alleinstellungsmerkmal des Nationalfonds in der Tatsache, dass er als einzige Organisation, die in diesem Bereich tätig ist, beim Parlament angesiedelt ist (vgl. Int.3, S.9, Z.305-307). Ein weiteres besonderes Merkmal des Nationalfonds läge auch in der langen Dauer seines Bestehens im Verhältnis zu anderen, ähnlichen Organisationen. Dennoch sei der Nationalfonds überraschend wenig im Inland bekannt (vgl. Int.3, S.10, Z.332-335). Im Gegensatz zu dem von ihr formulierten geringeren Bekanntheitsgrad im Inland seien jedoch der internationale Bekanntheitsgrad und die internationale Vernetzung des Nationalfonds herausragend und würden ihrer Meinung nach ebenfalls als Alleinstellungsmerkmal gesehen werden können (vgl. Int.3, S.10, Z.339-341).

Durch den Nationalfonds sei ein positives Zeichen hinsichtlich der Wahrnehmung der Mitverantwortung Österreichs an den in der Vergangenheit begangenen Verbrechen gesetzt worden. Die befragte Mitarbeiterin hebt in diesem Zusammenhang vor allem die aktive und gelungene Kontaktherstellung mit den Antragstellerlnnen hervor (vgl. Int.4, S.4, Z.141-146).

Die gewachsene Struktur im Nationalfonds ist laut einer Mitarbeiterin einzigartig. Es habe keine vorgeformte strenge Führung, sondern viel Flexibilität und auch Entwicklungsmöglichkeiten für MitarbeiterInnen gegeben (vgl. Int.5, S.4-5, Z.146152). Einzigartig sei auch die Möglichkeit, die Führungsebene im Fonds zu erreichen, wenn man sie brauche. Die Mitarbeiterin empfindet die Nahbarkeit der Führungsebene als etwas, das den Fonds ebenfalls auszeichnen würde (vgl. Int.5, S.5, Z.156-158). 
Für Frau Mag. Lessing ist eine Besonderheit im Nationalfonds, dass auch von Seiten der Geschäftsleitung bisher keine allzu hierarchisch organisierte Struktur gewünscht war und dem noch immer so ist. Dies lasse sehr viel Freiraum für die Entwicklung der Mitarbeiterlnnen zu. Dennoch gibt sie zu bedenken, dass gewisse Hierarchien eingehalten werden sollten und sieht hier einen klaren Vorteil in der neuen Struktur (vgl. Lessing, S.6, Z.189-200).

Die Einzigartigkeit des Nationalfonds liege in der Definition des Opferbegriffs. Der Nationalfonds habe „[...] den breitesten Opferbegriff [...]“ (Int.6, S.7, Z.227) etabliert und zum ersten Mal verschiedene Opfergruppen entschädigt und so als Opfergruppen charakterisiert, betont eine Mitarbeiterin. Dies sei etwas, worauf man im Nationalfonds stolz sein könne (vgl. Int.6, S.7, Z.226-230). Der Nationalfonds würde auch aktiv von außen kontaktiert und als Partner für Kooperationen gesucht werden (vgl. Int.6, S.7, Z.235-236). Sie sieht die Besonderheit des Nationalfonds nicht nur in seinen Kompetenzen, sondern auch in seiner Verankerung. Der Nationalfonds sei kein Verein, sondern eine Institution und damit im Gegensatz zu anderen Vereinen kaum angreifbar (vgl. Int.6, S.7, Z.240-244). Für die Mitarbeiterin liegt ein Alleinstellungsmerkmal des Nationalfonds ebenfalls in seiner Ansiedelung beim Parlament (vgl. Int.6, S.7, Z.249).

Für Frau Mag. Lessing liegt die Besonderheit des Nationalfonds ebenfalls in der gesetzlichen Definition der Opfergruppen:

$[\ldots]$ ich glaube, das Einzigartige daran ist, $[\ldots]$ dass wir alle Opfergruppen vertreten, dass sie alle zu uns ein sehr großes Vertrauen gewonnen haben, dass Roma und Sinti, Juden, Kinder vom Spiegelgrund sich gleichermaßen bei uns wirklich aufgehoben gefühlt haben und fühlen und wir [..] hatten die einzigartige Chance, seit 1995 mit über 30.000 Überlebenden arbeiten zu dürfen. (Lessing, S.8, Z.264-268)

Damit beschreibt sie die Unterstützung der Überlebenden als Privileg. Für sie ist der direkte Kontakt mit den Opfern das, was den Nationalfonds ausmacht. Ihr ist keine andere Organisation bekannt, die in gleichem Maß mit den Überlebenden gearbeitet 
hat und sie sieht es als etwas Besonderes an, dass so viele Überlebende dem Nationalfonds ihre Erinnerungen und Lebensgeschichten anvertraut haben (vgl. Lessing, S.8, Z.268-277).

Sowohl die Einrichtung des Nationalfonds bei der Parlamentsdirektion, als auch die Tatsache, dass die ersten Antragstellerlnnen in den Räumlichkeiten des Parlaments empfangen wurden, nahm Frau Dr. Meissner als besondere Anerkennung für die Antragstellerlnnen wahr (vgl. Meissner, S.5-6, Z.177-190). Den Nationalfonds zeichne auch nach Meinung von Frau Dr. Meissner vor allem die Arbeit mit den Überlebenden aus.

Einzigartig, vielleicht darüber hinausgehend, [...] ist, dass der Nationalfonds mit, ich glaube über 15.000, 16.000, 17.000 Personen, die die Verfolgung auf unterschiedlichste Weise erlebt haben, persönlich sprechen konnte, sich die Mitarbeiter ein wahnsinniges Wissen aneignen konnten, das durch die Anträge und die Registratur, die wir haben und die Dokumente, die Nationalfonds und Entschädigungsfonds durch die Jahre zur Dokumentation der Anträge zusammengetragen haben, einen unglaublichen Schatz bietet [...] (Meissner, S.19, Z.680-686)

Überdies stelle das Wissen der MitarbeiterInnen, welches in Workshops zu Wissensmanagement und Wissenserhalt zur Verfahrensbearbeitung und Arbeitsabläufen dokumentiert wurde, eine Besonderheit dar. (vgl. Meissner, S.19, Z.690-700).

\section{Veränderungen der Aufgabengebiete}

Die Veränderungen der Aufgabengebiete und die damit zusammenhängenden Herausforderungen wurden in einer weiteren Subkategorie erfasst. Arbeitsbereiche haben sich, wie bereits in der Literatur erläutert, im Laufe der Jahre seit der Gründung des Nationalfonds verändert. 
Auf die Frage nach der Veränderung des Nationalfonds von einem kleinen Büro hin zu einer großen Organisation, antwortet eine Mitarbeiterin, dass während dieser Veränderungen immer fraglich war, wie lange der Fonds überhaupt bestehen würde (vgl. Int.1, S.3, Z.87-88). Aufgabenbereiche haben sich laufend verändert. Parallel zur Gründung des Allgemeinen Entschädigungsfonds kam im Nationalfonds im Jahr 2001 die Aufgabe der Mietrechtsentschädigung hinzu (vgl. Int.1, S.5, Z.154-161).

Die weiteren Entschädigungszahlungen wie die Mietrechtsentschädigung durch den Nationalfonds und weitere Vermögensentschädigungen durch den Allgemeinen Entschädigungsfonds haben zu einer Personalaufstockung geführt (vgl. Int.3, S.2, Z.49-55). Dieses rasche Wachstum war eine Herausforderung für die Organisation, die nicht gänzlich gelungen sei. Durch den Fokus auf die Erledigung der Arbeit wurden kaum teamstärkende Maßnahmen ergriffen (vgl. Int.3, S.3, Z.75-90). Diese Veränderung in der Größe der Organisation wirke bis heute nach: "Dann kam das rasche Anwachsen mit sehr vielen neuen Mitarbeitern, die sich allein gelassen gefühlt haben. Das hat bewirkt, dass da ein Bruch entstanden ist, [...] der auch geblieben ist, glaube ich." (Int.3, S.3, Z.90-92)

Durch Improvisation und auch Eigeninitiative der MitarbeiterInnen habe sich vieles verändert, erzählt ein Mitarbeiter. Er verweist auf die fehlende Technik zu Beginn der Arbeit des Nationalfonds (vgl. Int.2, S.3, Z.103-107). Die Arbeit habe sich durch die Veränderung der Technologie gewandelt. Es bestehe heute ein höherer qualitativer Anspruch an die Tätigkeiten (vgl. Int.2, S.3-4, Z.109-116). Er berichtet, dass sich Abteilungen im Laufe der Zeit verändert haben, die Datenerfassung und die juristischen Abteilungen wurden verkleinert und auch die Arbeit an sich veränderte sich (vgl. Int.2, S.4, Z.130-132). Eine Veränderung in der Organisation bestand auch im Entstehen von neuen Aufgabenbereichen: "[...] die Lebensgeschichten, die Familienrecherche, das Findbuch, die Kunstdatenbank. Das war ja nicht intendiert [...]" (Int.2, S.4, Z.142-143) Er beschreibt damit Veränderungen und Erweiterungen von Arbeitsgebieten, die von innen heraus entstanden sind und $z u$ einer Spezialisierung in der Organisation geführt haben (vgl. Int.2, S.4, Z.140-149). Er erzählt, dass viele Projekte von MitarbeiterInnen in Eigeninitiative begonnen wurden und es eine Herausforderung darstellte, diese Projekte und Aufgaben erfolgreich zu implementieren (vgl. Int.2, S.16, Z.575-580). 
Eine weitere Mitarbeiterin sieht die Veränderung der Aufgabenbereiche im Nationalfonds ebenfalls bedingt durch die Entwicklung der Organisation weg von einer Auszahlungsstelle hin zu einer Förderstelle und zu einer Wissensvermittlungsund Beratungsstelle (vgl. Int.5, S.1-2, Z.37-42). Die Veränderungen in einer Organisation würden die Arbeit interessant machen, weil man immer wieder mit neuen Aufgaben konfrontiert sei. Dies würde aber auch Anpassungsfähigkeit und Flexibilität von MitarbeiterInnen erfordern (vgl. Int.5, S.2, Z.47-51).

Für die neuen Aufgaben, die durch die Gesetzesnovelle implementiert wurden, ist es einer Mitarbeiterin nach sehr wichtig, dass ein Netzwerk geschaffen wird. "[...] ich finde, dass ein Netzwerk und zwar generell, nicht nur [...] innerhalb [...] einer Organisation, sondern auch rundherum geschaffen werden soll, damit es gut funktioniert." (Int.6, S.3, Z.91-93)

Eine der Aufgaben, die sich verändert haben, war laut Frau Mag. Lessing die Projektförderung. "Die Projektförderungen habe ich damals geleitet, [...] das war [...] noch sehr in den Kinderschuhen, das hat sich dann erst später wirklich zu einer sehr massiven Projektförderung entwickelt." (Lessing, S.3, Z.98-100) Frau Mag. Lessing sieht die Veränderung der Aufgabenbereiche auch im Abebben des Parteienverkehrs. Menschen zu empfangen und innen zuzuhören stellte über lange Jahre hinweg die Hauptaufgabe des Nationalfonds und auch des Allgemeinen Entschädigungsfonds dar (vgl. Lessing, S.3, Z.100-104).

Eine Entwicklung fand auch in der Recherche statt. Mag. Lessing berichtet, dass die Recherche im Laufe der Jahre immer differenzierter und detaillierter wurde, auch durch die Entwicklung der Internetrecherche (vgl. Lessing, S.3-4, Z.104-112). Mit der Zeit kamen immer neue Aufgaben hinzu. Die Arbeit wurde komplexer (vgl. Lessing, S.4, Z.112-116).

Mag. Lessing sieht aktuell die Notwendigkeit einer stärkeren Vernetzung mit anderen Organisationen. Auf die neue Unternehmensstruktur bezogen, betont sie hier den Bereich der Vermittlung. Man müsse aus der langjährigen Arbeit lernen und die aktuellen gesellschaftlichen Entwicklungen miteinbeziehen. Radikalisierung und Antisemitismus seien wieder Thema. Diesbezüglich müsse man neue Wege in der 
Vermittlung suchen und weiterhin auch kreativ denken. Diese Kreativität sei immer eine Stärke des Nationalfonds gewesen, wie Frau Mag. Lessing erläutert (vgl. Lessing, S.6, Z.211-221).

Laut Frau Dr. Meissner wurde der bildungspolitische Auftrag immer "[...] implizit mitgefühlt [...]." (Meissner, S.9, Z.319) Durch das Engagement der MitarbeiterInnen, durch die intensive Arbeit mit den AntragstellerInnen und Opfervertretungen wurden dem Nationalfonds immer wieder neue Aufgaben übertragen "[...] und wir haben auch selbst begonnen, Dinge die uns wichtig waren, soweit es unsere Ressourcen ermöglicht haben, mitlaufen zu lassen [...]." (Meissner, S.9, Z.314-315)

Eine wesentliche Veränderung brachte die dauerhafte Einrichtung des Nationalfonds, wie Frau Dr. Meissner festhält. Auch sie erzählt, dass der Nationalfonds ursprünglich eine temporäre Institution mit nur wenigen, im Gesetz verankerten, Aufgaben, nämlich die Gestezahlungen, die Zusatzzahlungen für sozial schwache Personen und die Projektförderung sein sollte (vgl. Meissner, S.9, Z.304-310). Zusätzlich zu diesen Aufgaben habe man aber bereits von Beginn an weitere Aufgaben erfüllt, schildert sie. Die Bewahrung der Lebensgeschichten der Antragstellerlnnen im Sinne eines bildungspolitischen Auftrags wurde von Anfang an als wichtig erachtet. Auch hier zeigte sich wieder das Engagement und die Eigeninitiative der Mitarbeiterlnnen (vgl. Meissner, S.9, Z.310-319).

Laut Dr. Meissner hat sich in den 24 Jahren des Bestehens viel geändert. Durch die letzte Gesetzesnovelle wurden auch Aufgaben verankert, die bereits vorab als wesentlich erachtet wurden.

Also geändert hat sich wirklich viel und aus heutiger Sicht, fast vor dem 25jährigem Jubiläum, haben wir das, was wir uns eigentlich gewunschen [sic] und für wichtig erachtet haben, [...] jetzt auch gesetzlich umsetzen können und das ist wirklich ein fantastischer Weg [...]. (Meissner, S.9, Z.326-329)

Für sie war der Nationalfonds nie eine reine Auszahlungsstelle, sondern immer schon eine Institution und Servicestelle für Menschen, nicht nur für Überlebende, sondern auch für die nachfolgenden Generationen. Dies würde sich auch in der 
Vermittlungsarbeit des Nationalfonds an Schulen zeigen (vgl. Meissner, S.10, Z.334341).

Ähnlich einer befragten Mitarbeiterin beschreibt Frau Dr. Meissner die Wandlung von einer reinen Auszahlungsstelle zu einer Vermittlungsstelle für Wissenschaft und Aufarbeitung als zentrale Veränderung in der Organisation, auch im Sinne des bereits erwähnten bildungspolitischen Auftrags des Nationalfonds (vgl. Meissner, S.9-10, Z.329-344). Die Aufarbeitung der gesammelten Materialien im Archiv des Nationalfonds stellt für Frau Dr. Meissner einen neuen Arbeitsbereich und auch eine Herausforderung für die Zukunft dar. Sie bezeichnet die gesammelten Unterlagen als "[...] Schatz und jetzt geht es darum diesen Schatz [...] nach datenschutzrechtlichen Bedingungen [...] für die Nachwelt und Wissenschaft wirklich aufzubereiten." (Meissner, S.10, Z.345-347)

Auch die Gründung und die baldige Beendigung des Allgemeinen Entschädigungsfonds nähmen eine zentrale Rolle in der Veränderung der Organisation ein. Es seien durch etliche Gesetzesnovellen und durch das Washingtoner Abkommen immer wieder weitere Aufgabengebiete hinzugekommen. Die Vorgabe, „[...] dass der Entschädigungsfonds eigentlich unter den Auspizien des Nationalfonds arbeitet [...]" (Meissner, S.11, Z.402), hätte zu einer engen Vernetzung der beiden Fonds und in weiterer Folge zu einer Organisationsveränderung geführt (vgl. Meissner, S.11, Z.397-405).

\subsubsection{Kategorie 2: Gesetzesnovelle des Nationalfondsgesetzes 2017}

Diese Kategorie beschreibt in zwei Subkategorien einerseits die Entstehung des Nationalfondsgesetzes und dessen Besonderheiten und andererseits die letzte Gesetzesnovelle aus dem Jahr 2017. In der zweiten Subkategorie wird darauf eingegangen, welche Gründe zu dieser Gesetzesnovelle führten, welche Ergebnisse sich abzeichnen und welche Chancen die Gesetzesnovelle für die weitere Arbeit des Nationalfonds beinhaltet. 


\section{Besonderheiten des Nationalfondsgesetzes}

Im Rahmen der Erstellung des Nationalfondsgesetzes gab es Diskussionen hinsichtlich des Umfangs der Entschädigungsleistungen, erinnert sich Frau Dr. Janistyn-Novák. Es wurde debattiert, ob individuelle Leistungen oder Pauschalleistungen erbracht werden sollten. Dr. Janistyn-Novák vermutet, dass der Nationalfonds gescheitert wäre, wenn man sich nicht für einen allgemeinen Ansatz der Anerkennung des Leids entschieden hätte (vgl. Janistyn-Novák, S.2, Z.59-65). Eine Vergleichbarkeit der Entschädigung der unterschiedlichen Verfolgungsschicksale konnte nicht angestrebt werden, daher einigte man sich auf Pauschalleistungen (vgl. Janistyn-Novák, S.2, Z.65-69).

Das Gesetz würde das Gewissen Österreichs reflektieren, erzählt Frau Mag. Lessing. Sämtliche Gesetzesnovellierungen seien im Laufe der Jahre immer einstimmig beschlossen worden, dies verdeutliche eine Anerkennung des Nationalfonds. "Wir sind keine Organisation, die irgendwo angesiedelt ist, sondern wir sind beim Nationalrat angesiedelt und das kann ich nicht oft genug sagen, dass das sozusagen wirklich im Herzen der Republik ist [...]." (Lessing, S.7, Z.238-240)

Für Frau Dr. Meissner ist bedeutend, dass erstmals verschiedene Opfergruppen im Nationalfondsgesetz festgeschrieben wurden (vgl. Meissner, S.3, Z.86). "[...] das war das Wesentliche, erstmals wirklich ein ganz, ganz breites Spektrum von den Opfergruppen, die damals historisch als Opfergruppen überhaupt belegt waren und von denen man auch wusste [...]" (Meissner, S.3, Z.91-94)

Der Verfassungsrang des Nationalfondsgesetzes macht den Nationalfonds einem Mitarbeiter zufolge einzigartig (vgl. Int.2, S.7-8, Z.258-263).

\section{Gründe, Formulierung und Ergebnis der Gesetzesnovelle, Chancen für den}

Nationalfonds in der Zukunft

Für Frau Dr. Janistyn-Novák ist die Gesetzesnovelle von 2017 der Versuch, eine Antwort auf eine Zukunft ohne ZeitzeugInnen zu finden. Für die nachfolgenden Generationen sei die Arbeit des Nationalfonds weiterhin wichtig, um die 
Erinnerungskultur und das Gedenken mit Leben zu füllen (vgl. Janistyn-Novák, S.9, Z.312-317).

Es sei essentiell für die konkrete Umsetzung der Gesetzesnovelle, dass Vorschläge für die zukünftigen Aufgaben vom Nationalfonds selbst kommen, betont Frau Dr. Janistyn-Novák (vgl. Janistyn-Novák, S.19, Z.678-681). Bezogen auf die zukünftigen Aufgaben des Nationalfonds sei die Formulierung der Novelle gut gelungen (vgl. Janistyn-Novák, S.1, Z.28-30).

Frau Mag. Maschke sieht es als Chance und auch als positive Herausforderung, dass die neuen Aufgaben des Nationalfonds in der Gesetzesnovelle verankert wurden (vgl. Maschke, S.3, Z.91-100).

Mit der Gesetzesnovelle sei eine gute Grundlage und Präzision für die kommenden Aufgaben geschaffen worden, betont auch ein Mitarbeiter (vgl. Int.2, S.5, Z.164-165). Er hält fest, dass nun durch diese Novelle eine nachträgliche Legitimation der Aufgaben, die teilweise bereits erfüllt werden, stattfand (vgl. Int.2, S.5, Z.179-180). Es sei auch intendiert, dass die Gesetzesnovelle in der neuen Organisationsstruktur abgebildet sei (vgl. Int.2, S.6, Z.186-187).

Eine andere Mitarbeiterin bezeichnet die Gesetzesnovelle von 2017 ebenfalls als eine Initiative vom Nationalfonds zur Konkretisierung von Aufgaben, die teilweise bereits übernommen wurden (vgl. Int.3, S.6, Z.195-198). Die Novelle hat nach Angaben der Mitarbeiterin eine Lücke in der gesetzlichen Legitimierung von Aufgabenbereichen geschlossen (vgl. Int.3, S.6, Z.198-203). Sie sieht in der neuen Struktur der Organisation und den neuen Aufgabenbereichen ebenfalls eine Abbildung der Novelle (vgl. Int.3, S.7, Z.244-247). Die Gesetzesnovelle würde auch den Weg für die zukünftige Vermittlungsarbeit ebnen, vor allem in Hinblick auf die Vermittlungsarbeit an Schulen und mit Jugendlichen (vgl. Int.3, S.15-16, Z.546-550).

Der langsame Wegfall von Agenden, wie die Zahlungen an Antragstellerlnnen und die Erweiterung des Betätigungsfeldes, waren laut einer anderen Mitarbeiterin ebenfalls Gründe für die Gesetzesnovelle (vgl. Int.4, S.2, Z.69-74). 
Für eine weitere Mitarbeiterin bietet die Formulierung der Gesetzesnovelle auch die Sicherheit für die öffentliche Bekanntmachung verschiedener Aufgabenbereiche (vgl. Int.5, S.2, Z.67-71). Die Novelle würde außerdem das Gefühl von Sicherheit für das Bestehen des Nationalfonds vermitteln, erzählt sie und sieht darin eine Zukunftsorientierung (vgl. Int.5, S.3, Z.75-79). Sie biete auch allgemein für MitarbeiterInnen Sicherheit und Struktur (vgl. Int.5, S.3, Z.84-86). Eine Abbildung der Gesetzesnovelle in der neuen Organisationsstruktur würde durch das neue Organigramm ersichtlich werden (vgl. Int.5, S.3, Z.91-102).

Die Veränderungen in der Arbeit, die mit der Gesetzesnovelle einhergehen, werden von einer anderen Mitarbeiterin beschrieben. Früher konnte man Rechercheergebnisse von Familiengeschichten nur dem Kreis der Antragsstellerlnnen zugänglich machen. Durch die Novelle sei es auch möglich, Anfragen von nachfolgenden Generationen nachzukommen. Hier würde die Novelle die Chance bieten, Menschen auf der Suche nach ihrer Vergangenheit und ihrer Familiengeschichten behilflich zu sein (vgl. Int.6, S.4-5, Z.143-165).

Frau Mag. Lessing beschreibt die Gesetzesnovelle als eine notwendige gesetzliche Implementierung von Aufgaben, die teilweise bereits ausgeführt wurden, für die es jedoch noch keine gesetzliche Grundlage gab. Für Frau Mag. Lessing diente die Novelle der gesetzlichen Legitimierung sämtlicher Tätigkeiten des Nationalfonds (vgl. Lessing, S.4, Z.132-135).

Einer der vorrangigen Gründe für die Gesetzesnovelle war für Frau Dr. Meissner die gesetzliche Verankerung des Projekts der Neugestaltung der österreichischen Länderausstellung in Auschwitz- Birkenau. Diese Aufgabe wurde dem Nationalfonds durch einen Ministerratsvortrag übertragen und musste durch ein Gesetz konsolidiert werden (vgl. Meissner, S.10, Z.354-361).

Auch für Frau Dr. Meissner deckt die Novelle nun alle Aufgaben ab, die bereits teilweise erfüllt wurden (vgl. Meissner, S.11, Z.370-376). Sie sei der Grundstein für weitere ausbaufähige Aufgabenbereiche, die der Nationalfonds in Zukunft übernehmen kann (vgl. Meissner, S.11, Z.382-393). In der neuen Organisationsstruktur wurde auch nach Frau Dr. Meissner versucht, die 
Gesetzesnovelle mit den Alleinstellungsmerkmalen des Nationalfonds und seiner zukünftigen Aufgaben abzubilden (vgl. Meissner, S.14, Z.500-503).

\subsubsection{Kategorie 3: Umstrukturierung des Nationalfonds 2019}

In dieser Kategorie ergaben sich anhand der ausgewerteten Antworten der InterviewpartnerInnen drei Subkategorien, welche die Umstrukturierung näher beleuchten. Die Gründe für die Umstrukturierung werden beschrieben, ebenso die Veränderungen, die damit einhergehen und welche Auswirkungen bereits für Mitarbeiterlnnen zu spüren sind. Des Weiteren wird ein Bezug zur Gesetzesnovelle hergestellt.

\section{Gründe, Veränderungen und Auswirkungen}

Frau Dr. Meissner schildert die Zusammensetzung der neuen Struktur, die auf sechs Säulen basiert. Die erste Säule ist der Bereich "Opferanerkennung, Kommunikation und Öffentlichkeitsarbeit", die zweite Säule ist nach wie vor die Projektförderung, die seit Beginn des Nationalfonds ausgeführt wird und die dritte Säule ist ein neuer Bereich, der unter dem Namen "Wissenschaft, Gedenken und Vermittlung" laufen wird (vgl. Meissner, S.14-15, Z.517-528). Ein vierter Bereich namens „Historische Recherchen, Kunstrestitution und Findbuch sowie Registratur/Archiv" wurde aus bereits bestehenden Abteilungen gebildet. Die zukünftigen und derzeitigen Aufgaben des Nationalfonds werden in diesen vier Bereichen abgebildet. Die anderen zwei Säulen bilden die bisher bereits existierenden Supportabteilungen „Personal \& Finanzen“ und „IT“. Für Frau Dr. Meissner liegt der Vorteil der neuen Struktur darin, dass es keine spezifischen Abteilungen mehr geben wird, sondern Bereiche, in denen übergreifend zusammengearbeitet werden wird (vgl. Meissner, S.15, Z.532544).

Die Herausforderung der Reorganisation laut Frau Dr. Meissner war, in einem ersten Schritt nicht auf die persönlichen Fähigkeiten und Kompetenzen der Mitarbeiterlnnen Rücksicht nehmen zu können. Abteilungen wurden aufgelöst und zu größeren Bereichen zusammengelegt, dadurch entfielen auch Führungsaufgaben. Dies sei für MitarbeiterInnen natürlich keine einfache Situation (vgl. Meissner, S.16, Z.558-567) Es wurde jedoch laut Frau Dr. Meissner darauf geachtet, dass es Synergieeffekte in 
den neuen Bereichen geben wird. Die Mitarbeiterlnnen müssten sich in den neuen Bereichen erst zurechtfinden (vgl. Meissner, S.16, Z.567-572)

Die Umstrukturierung bietet laut Dr. Meissner wieder die Möglichkeit, sich auf die inhaltliche Arbeit zu fokussieren und auch etwaige Doppelrollen hinsichtlich Funktionen und Positionen der MitarbeiterInnen zu vermeiden. Die Veränderung der Aufgabenbereiche ermöglicht somit wieder mehr Struktur (vgl. Meissner, S.13, Z.475477). In der Gesetzesnovelle von 2017 wurden teilweise Aufgaben gesetzlich verankert, die der Nationalfonds bereits seit Jahren erfüllt, wie z.B. die Neugestaltung der Österreich-Ausstellung in Auschwitz-Birkenau, aber auch neue Aufgaben wurden implementiert, wie der Bereich 'Wissenschaft, Gedenken und Vermittlung' (vgl. Meissner, S.13-14, Z.477-490). Für Dr. Meissner ist hier "[...] eine wunderschöne Aufgabe hinzugekommen [...]." (Meissner, S.14, Z.491)

Frau Dr. Meissner sieht die Notwendigkeit der Umstrukturierung in Zusammenhang mit der bevorstehenden Beendigung des Entschädigungsfonds Ende 2019. Die zukünftige Arbeit werde sich auf die Aufgaben, die bereits 2017 in der Gesetzesnovellierung formuliert wurden, konzentrieren (vgl. Meissner, S.12-13, Z.441-446).

Ein Teil des Umstrukturierungsprozesses betraf die Abbildung von temporären Tätigkeitsbereichen im neuen Organigramm (vgl. Meissner, S.16-17, Z.585-593). Der Fonds zur Instandsetzung der jüdischen Friedhöfe in Österreich, der für 20 Jahre beim Nationalfonds eingerichtet wurde, und das Projekt der Neugestaltung der österreichischen Länderausstellung in Auschwitz-Birkenau wurden als Stabstellen in die neue Struktur eingegliedert. Für die Abwicklungsagenden der noch offenen Aufgaben nach seiner offiziellen Beendigung Ende 2019 wird der Allgemeine Entschädigungsfonds ab Jänner 2020 ebenfalls als Stabstelle eingegliedert werden (vgl. Meissner, S.17, Z.601-618).

Den Erläuterungen von Frau Dr. Meissner folgend, werden die neuen Bereiche und Stabstellen in den beiden folgenden Abbildungen, welche im Rahmen einer Mitarbeiterlnnenversammlung am 14. Mai 2019 präsentiert wurden, anschaulich dargestellt. 


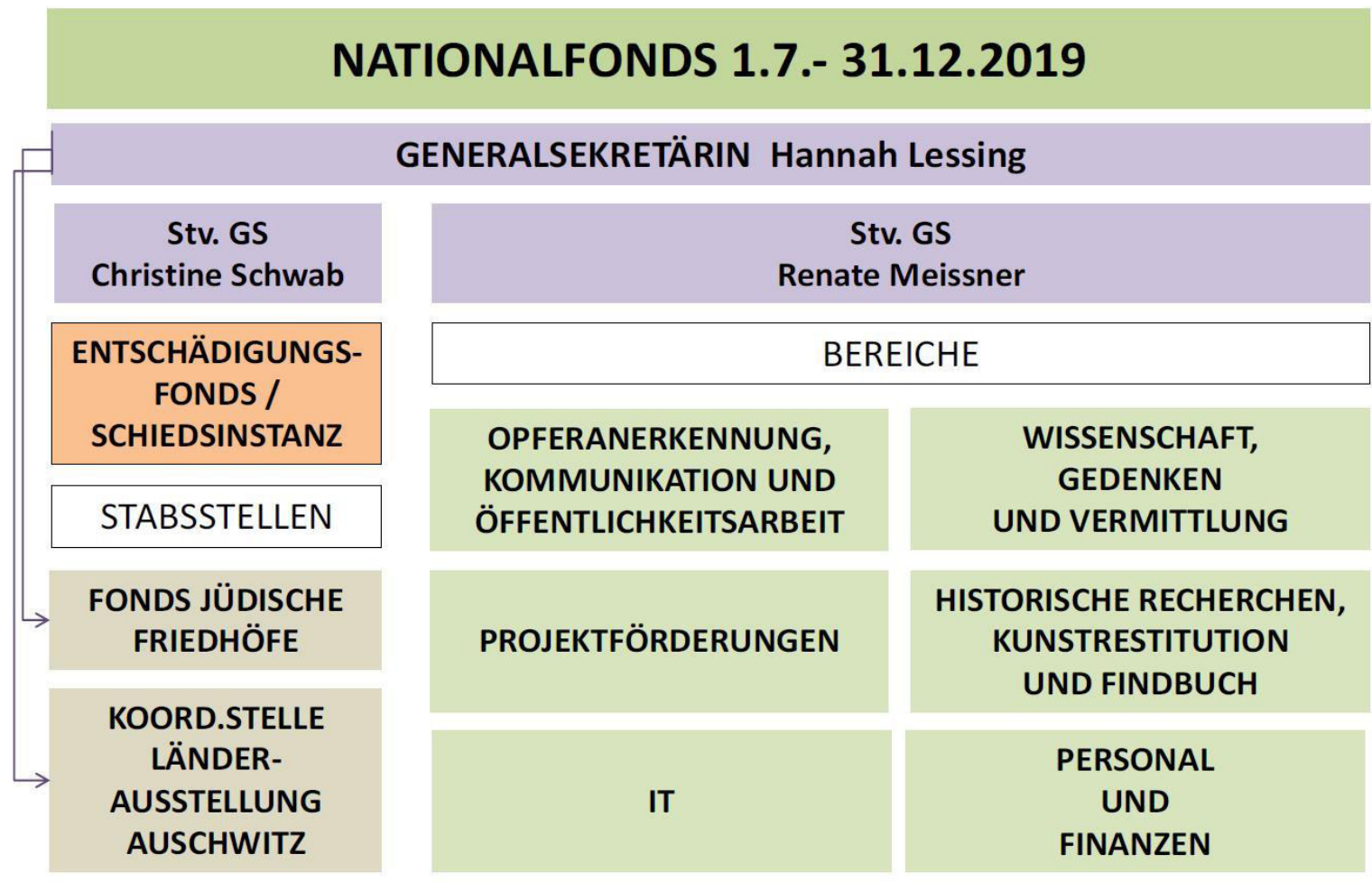

Abb.2: Die neue Organisationsstruktur im Überblick in der Übergangsphase bis 31.12.2019. (Quelle: Präsentation Organisationsstruktur, 2019)

\section{NATIONALFONDS ab $\mathbf{1 . 1 . 2 0 2 0}$}

\begin{tabular}{|c|c|c|}
\hline \multicolumn{3}{|c|}{ GENERALSEKRETÄRIN Hannah Lessing } \\
\hline & \multicolumn{2}{|c|}{$\begin{array}{c}\text { Stv. GS } \\
\text { Renate Meissner }\end{array}$} \\
\hline STABSSTELLEN & \multicolumn{2}{|c|}{ BEREICHE } \\
\hline $\begin{array}{l}\text { ENTSCHÄDIGUNGS- } \\
\text { FONDS / } \\
\text { SCHIEDSINSTANZ }\end{array}$ & $\begin{array}{l}\text { OPFERANERKENNUNG, } \\
\text { KOMMUNIKATION UND } \\
\text { ÖFFENTLICHKEITSARBEIT }\end{array}$ & $\begin{array}{l}\text { WISSENSCHAFT, } \\
\text { GEDENKEN } \\
\text { UND VERMITTLUNG }\end{array}$ \\
\hline $\begin{array}{l}\text { FONDS JÜDISCHE } \\
\text { FRIEDHÖFE }\end{array}$ & PROJEKTFÖRDERUNGEN & $\begin{array}{l}\text { HISTORISCHE RECHERCHEN, } \\
\text { KUNSTRESTITUTION } \\
\text { UND FINDBUCH }\end{array}$ \\
\hline $\begin{array}{l}\text { KOORD.STELLE } \\
\text { LÄNDER- } \\
\text { AUSSTELLUNG } \\
\text { AUSCHWITZ }\end{array}$ & IT & $\begin{array}{l}\text { PERSONAL } \\
\text { UND } \\
\text { FINANZEN }\end{array}$ \\
\hline
\end{tabular}

Abb.3: Die neue Organisationsstruktur im Überblick ab 1.1.2020. (Quelle: Präsentation Organisationsstruktur, 2019) 
Die Umstrukturierung hatte verschiedene Auswirkungen auf Mitarbeiterlnnen. Es bedeutete Veränderung und wurde von manchen mit Stress oder auch Verständnislosigkeit aufgenommen. Andere wiederum begrüßten diese Veränderung sehr, wie eine Mitarbeiterin berichtet (vgl. Int.1, S.5, Z.183-185). Auch ein anderer Mitarbeiter merkt an, dass die Umstrukturierung Widerstände durch das Aufbrechen von alten und gewohnten Strukturen hervorrufen würde. Seiner Meinung nach erfordere es jedoch eine Umstellung und eine Professionalisierung der Arbeit und der Arbeitsabläufe (vgl. Int.2, S.6, Z.194-202).

Für eine weitere Mitarbeiterin steht vor allem die Teambildung in den neuen Bereichen im Vordergrund. Eine Stabilisierung der neuen Teams müsse gelingen. Ihrer Meinung nach braucht es in der Organisation auch eine Art von Aufarbeitung von alten Defiziten. Es sei bemerkenswert, wie die Organisation gewachsen ist und nun wieder verkleinert werde. Diese Umstellung erfordere auch Geduld (vgl. Int.3, S.3, Z.102-111). Auch im Allgemeinen Entschädigungsfonds habe es von Anfang an wenig vorgegebene Strukturen gegeben, diese seien durch Eigeninitiative der Mitarbeiterlnnen selbst eingeführt worden. Diese Selbstständigkeit werde durch die neue Struktur nach der Umstrukturierung begrenzt, es solle mehr Leitung implementiert werden, diese Umstellung sei schwierig für Mitarbeiterlnnen (vgl. Int.3, S.4, Z.135-147). MitarbeiterInnen würden die Umstrukturierung unterschiedlich wahrnehmen. Für manche sei die Notwendigkeit der Umstellung ersichtlich, andere fühlen sich in ihrer Selbstständigkeit nun eingeschränkt und sähen die vorgenommenen Änderungen als Kritik an der bisherigen Arbeit (vgl. Int.3, S.5, Z.151-157). Der Nationalfonds hätte fast ein familiäres Ambiente, wie die Mitarbeiterin berichtet. Es gebe keine konzernartigen Strukturen und kein großes Gefälle zwischen Dienstgeber und Dienstnehmer, dies würde den Umgang mit der Umstellung ebenfalls erschweren (vgl. Int.3, S.5, Z.170-177). Die Mitarbeiterin erzählt, dass durch die durchwegs amikalen Umgangsformen im Nationalfonds der Eindruck erweckt werden könne, es gäbe keine oder nur kaum eine Führung. Dem sei aber nicht so (vgl. Int.3, S.5, Z.181-183).

Die Umstrukturierung müsse daher im Sinne der zukünftigen Zusammenarbeit gelingen. Es müssten Bereiche geschaffen werden, in denen eine "[...] relativ enge übergreifende Zusammenarbeit zwischen den Bereichen [...]" (Int.3, S.7, Z.248-249) 
gewährleistet sei. Es brauche mehr Übersicht und Effizienz, damit bisherige Doppelrollen und Unklarheiten in Zukunft beseitigt werden könnten (vgl. Int.3, S.7, Z.247-257). Für die Mitarbeiterin ist ein Ergebnis des raschen Wachstums der Organisation der Mangel an Transparenz zwischen den einzelnen Abteilungen. Viele MitarbeiterInnen würden kaum über die Arbeitsbereiche der KollegInnen Bescheid wissen. Die große Herausforderung werde sein, im Zuge der Umstrukturierung eine konstante Führung zu etablieren und einen strukturierten Austausch zu schaffen (vgl. Int.3, S.7-8, Z.257-272). Für MitarbeiterInnen sei es "[...] ein Schock, ein Herausgerissen-werden aus sehr vertrauten Strukturen." (Int.3, S.8, Z.277) Durch den Bruch in der Tradition würden Ängste freigesetzt werden. Die Herausforderung für die Führung sei nun, durch die Umstrukturierung klare Linien zu setzen und so auch Sicherheit zu schaffen (vgl. Int.3, S.8, Z.278-288).

Auch einer anderen Mitarbeiterin zufolge müsse sich die Arbeitsstruktur ändern, das vorhandene Wissen und die Kompetenzen der Mitarbeiterlnnen sollten aber dennoch in der neuen Struktur Beachtung finden (vgl. Int.4, S.3, Z.82-88).

Fortbildungsmöglichkeiten, um den neuen Aufgaben gewachsen zu sein, hält die befragte Mitarbeiterin für essentiell (vgl. Int.4, S.3, Z.96-100). Sie ist skeptisch, ob es der neuen Führungsebene gelingen kann, die neuen Strukturen in dem vorgegebenen Zeithorizont umsetzen zu können. Ihrer Meinung nach ist die Belegschaft zu wenig miteinbezogen worden (vgl. Int.4, S.3, Z.109-112). Laut ihr hat sich die Umstrukturierung hauptsächlich auf einen Wechsel in der Führungsebene bezogen (vgl. Int.4, S.3-4, Z.112-119).

Für eine weitere Mitarbeiterin ist es fraglich, ob die bestehenden Netzwerke zwischen den früheren Abteilungen in der neuen Struktur weitergeführt werden können. Ihrer Ansicht nach wurden die Mitarbeiterlnnen zu wenig zu den bisher bestehenden Arbeitsabläufen befragt (vgl. Int.5, S.3-4, Z.106-112). Für die zukünftige Zusammenarbeit zwischen den neuen Bereichsleiterlnnen und den Mitarbeiterlnnen seien Kommunikation und ein Austausch über frühere Arbeitsabläufe und zukünftige Optimierungen von Vernetzungen besonders wichtig (vgl. Int.5, S.4, Z.116-119). Der Prozess der Umstrukturierung habe einen Bruch in der Kommunikation und der Belegschaft erzeugt (vgl. Int.5, S.4, Z.139-142). 
Auch eine andere Mitarbeiterin steht der Umstrukturierung skeptisch gegenüber. Die Unterstützung von anderen Organisationen, die die Wichtigkeit der Arbeit im Fonds betonen, motiviere sie jedoch (vgl. Int.6, S.6, Z.191-195). Sie spricht auch die fehlende Kommunikation zwischen den Abteilungen an, man wisse nicht, wer welche Aufgaben hat und welche Außenkontakte der verschiedenen Abteilungen es gebe. Die Geschäftsleitung habe für die Umstrukturierung zu wenig Informationen über die bisherigen Zuständigkeiten und Aufgaben eingeholt (vgl. Int.6, S.6, Z.200-209).

Ansätze einer Struktur sind immer mitgewachsen. Erst durch den Wegfall vieler Aufgaben im Allgemeinen Entschädigungsfonds wurde eine klare Struktur durch die Nationalfondsgesetzesnovelle eingeführt (vgl. Lessing, S.5, Z.159-165). Die Umstrukturierung bietet für Frau Mag. Lessing die Möglichkeit, selbst wieder mehr inhaltlich arbeiten zu können. Die Strukturen würden kleiner und überschaubarer werden und sie könne sich dadurch wieder mehr in Projekte einbringen (vgl. Lessing, S.5, Z.169-176). Für die Umstrukturierung hat sich die Geschäftsleitung Hilfe von außen durch eine Unternehmensberatung geholt. Die neuen Strukturen sollten transparent und nachvollziehbar gestaltet werden, dies wäre durch externe Hilfe gewährleistet gewesen, wie Frau Mag. Lessing erläutert (vgl. Lessing, S.5-6, Z.181184).

Mit der neuen Struktur würden wieder gewisse Hierarchien eingeführt. Freiraum für die Entwicklung der MitarbeiterInnen solle dennoch beibehalten werden (vgl. Lessing, S.6, Z.189-194). Durch die neue Struktur solle auch mehr Transparenz über Zuständigkeiten eingeführt werden, um Missverständnissen vorzubeugen. Dennoch sei es auch weiterhin möglich, bereichsübergreifend zu arbeiten und zu gestalten (vgl. Lessing, S.6, Z.194-200). Die neue Struktur biete viele Optionen für zukünftige Arbeitsbereiche, wie Frau Mag. Lessing ebenfalls erläutert. Vermittlungsarbeit und die Zusammenarbeit mit anderen Organisationen sollten wieder mehr ausgebaut werden (vgl. Lessing, S.6-7, Z.207-221). 


\section{Bezug zur Gesetzesnovelle 2017}

Mag. Lessing berichtet, dass es zwischen dem Nationalfonds und dem Allgemeinen Entschädigungsfonds immer personelle Verstrickungen gab. Durch die bevorstehende Beendigung des Allgemeinen Entschädigungsfonds musste der Nationalfonds neu strukturiert werden (vgl. Lessing, S.4, Z.140-146). Die Gesetzesnovelle biete klare Strukturen für die zukünftige Arbeit des Nationalfonds (vgl. Lessing, S.4-5, Z.147-149). Die neue Struktur war nötig, da im Laufe der Zeit Aufgaben weggefallen sind und die vorhandene Struktur nicht mehr passend erschien. Durch die Gesetzesnovelle konnte eine neue, auf dem Gesetz basierende, Struktur geschaffen werden (vgl. Lessing, S.5, Z.154-165).

Die Gesetzesnovelle war laut Frau Dr. Meissner der ausschlaggebende Grund für die Neuorganisation des Nationalfonds (vgl. Meissner, S.12-13, Z.441-446). Es war eine Herausforderung durch die baldige Beendigung des Entschädigungsfonds und einer damit zusammenhängenden Veränderung in der Belegschaft, den Nationalfonds für die Zukunft neu zu gestalten (vgl. Meissner, S.13, Z.458-461). „[...] wir haben wirklich versucht, die Alleinstellungsmerkmale des Nationalfonds mit seinen zukünftigen Aufgaben auch organisational abzubilden." (Meissner, S.14, Z.502-503)

Frau Mag. Maschke empfindet die Umstrukturierung und auch die Gesetzesnovelle als Chance für den Nationalfonds, sich neu aufzustellen. Durch die Veränderung des Aufgabenprofils ergäbe sich eine positive inhaltliche Herausforderung (vgl. Maschke, S.3, Z.91-100).

Die Aufgaben des Nationalfonds, die nach Meinung einer Mitarbeiterin in der Gesetzesnovelle abgebildet werden, bieten auch neue personelle Möglichkeiten, um die neuen Tätigkeiten abzudecken (vgl. Int.3, S.7, Z.244-247).

Es herrscht jedoch auch eine gewisse Skepsis, ob die Gesetzesnovelle tatsächlich in der neuen Struktur abgebildet ist. Einer Mitarbeiterin zufolge ist die Motivation und das Engagement der MitarbeiterInnen in der Planung der neuen Struktur nicht ausreichend beachtet worden (vgl. Int.6, S.5-6, Z.182-187). 


\subsubsection{Kategorie 4: Herausforderungen für den Nationalfonds}

Um ein umfassendes Bild der Herausforderungen für den Nationalfonds zu erhalten, wurde zuerst nach den Herausforderungen der Gründung und der ersten Zeit des Bestehens des Nationalfonds gefragt. Diesbezügliche Aussagen wurden in der ersten Subkategorie zusammengefasst. Des Weiteren wurde nach den Herausforderungen, die die Umstrukturierung mit sich bringt, gesondert gefragt. Die Aussagen hierzu ergeben die zweite Subkategorie.

\section{Herausforderungen nach der Gründung des Nationalfonds}

Für Frau Dr. Janistyn-Novák war eine der ersten Herausforderungen nach der Gründung des Nationalfonds die Bewertung der Verfolgung und der Entschädigungen. Zentrale Fragen waren für sie: "Wie kann man denn Verfolgung entschädigen? Was bedeutet das? Ist es quasi eine weniger starke Verfolgung, wenn jemandem gelungen ist, in den Zug einzusteigen und vielleicht nach Budapest noch auszuweichen?" (Janistyn-Novák, S.2, Z.69-71) Es war klar, dass in den Schicksalen der ungefähr 30.000 Überlebenden, die der Nationalfonds erreicht hat, keine Vergleichbarkeit angestrebt werden konnte (vgl. Janistyn-Novák, S.2, Z.65-69). Eine weitere Herausforderung war, dass mit Beschlussfassung des Gesetzes niemand wusste, wie viele Personen tatsächlich ausfindig gemacht werden könnten (vgl. Janistyn-Novák, S.3, Z.79-81). Einige Mitarbeiterinnen, die durch ihr Wissen und ihren offenen Zugang gute Lösungen ermöglicht haben, waren laut Dr. JanistynNovák wahre Leuchttürme. Dennoch seien es Kompromisslösungen gewesen. (vgl. Janistyn-Novák, S.3, Z.100-104)

Sehr herausfordernd war laut Dr. Janistyn-Novák auch der Druck, die Gestezahlungen so rasch wie möglich zu leisten. Zusätzlich zu dem Zeitdruck war auch die Frage nach der organisatorischen Abwicklung präsent (vgl. Janistyn-Novák, S.4, Z.143-147). Dr. Janistyn-Novák erinnert sich, dass ihr zu Beginn der Arbeit schnell klar wurde, dass sie Unterstützung brauchen würde. Zu ihren ersten Aufgaben zählte die Bekanntmachung einer offiziellen Telefonnummer. Bereits kurz nach der Beschlussfassung gingen schon etliche Anrufe ein. Zur Unterstützung konnte sie dann bereits im Sommer [1995, Anm. d. Verf.] Frau Dr. Meissner für die Aufgabe gewinnen (vgl. Janistyn-Novák, S.5, Z.154-162). Ab Sommer 1995 gab es 
dann eine erste Anlaufstelle im Parlament für die AntragstellerInnen (vgl. JanistynNovák, S.5, Z.163-164).

Wie bereits im Kapitel 4.3.1. unter der Subkategorie „Die Gründung und der Beginn des Nationalfonds" beschrieben, war die hohe Arbeitsbelastung in der Anfangszeit ein zentrales Thema. Da die befragten Personen den hohen Arbeitsaufwand auch bei der Frage nach den Herausforderungen nach der Gründung erwähnten, wird in diesem Kapitel nochmals erläuternd darauf eingegangen.

Eine der ersten Herausforderungen für Dr. Meissner waren die Orientierung und die Aneignung von Wissen zu Maßnahmen der Entschädigungen. Weitere Herausforderungen waren

[...] Leute zu finden, Struktur aufzubauen, Organisation aufzubauen, dann die psychische Herausforderung für die Mitarbeiterinnen, [...] ohne [...] wirklich gewappnet gewesen zu sein, was auf einen zukommt und die andere Herausforderung, die Leute überhaupt ausfindig zu machen, zu finden, das war dann eine der Hauptaufgaben der Mag. Lessing. (Meissner, S.7, Z.245249)

Dr. Janistyn-Novák erinnert sich an die hohe Arbeitsbelastung, die sich bereits kurze Zeit nach der Bekanntmachung des Nationalfonds zeigte. Aus heutiger Sicht hätten für die Arbeitsbewältigung von Beginn an mehr Mitarbeiterlnnen eingestellt werden sollen (vgl. Janistyn-Novák, S.5, Z.175-177). Trotzdem gelang es, rasch die ersten Zahlungen zu leisten. "[...] im Juni war die Beschlussfassung [...] und im Oktober waren tatsächlich die ersten Auszahlungen." (Janistyn-Novák, S.6, Z.199-201)

Die Errichtung des Nationalfonds wurde durch internationale Medien bekannt gemacht, um möglichst viele Menschen zu erreichen. Die Reisen und Vorträge von Frau Mag. Lessing haben ebenfalls zum internationalen Bekanntheitsgrad des Nationalfonds beigetragen (vgl. Meissner, S.7, Z.249-252). 
Die Belastung in der Anfangszeit zeigte sich laut Dr. Janistyn-Novák auch an der Menge der Post (siehe dazu auch Kapitel 4.3.1.), die an den Fonds geschickt wurde. Frau Dr. Janistyn-Novák erinnert sich, dass täglich drei Postsäcke eingetroffen sind und es aufgrund dieser Menge kaum möglich war, die Post per Hand zu öffnen. Es wurde daher eine Schneidemaschine zur Öffnung der Kuverts angeschafft und eine Mitarbeiterin öffnete tagtäglich ausschließlich Briefe (vgl. Janistyn-Novák, S.6, Z.185192). Eine Mitarbeiterin erzählt, dass sie zu Beginn ihrer Arbeit im Nationalfonds eine von mehreren StudentInnen war, die ausnahmslos Post geöffnet haben. In jedem Raum des damaligen Büros lagen Stapel von Briefen und Fragebögen, die von potentiellen AntragstellerInnen retourniert wurden (vgl. Int.6, S.1-2, Z.39-41). Der Arbeitsaufwand war eine große Herausforderung. Das Ausmaß der Arbeit wurde unterschätzt, es habe sich auch keiner der MitarbeiterInnen mit Organisationsstruktur beschäftigt, der Fokus lag auf der schnellen Erledigung der Arbeit (vgl. Int.6, S.1-2, Z.37-44). Die fehlende Organisationsstruktur hat auch zu einer langen Bearbeitungsdauer geführt. Eine Mitarbeiterin berichtet, dass es zu Beginn nur zwei Telefonleitungen gab und die Telefone rund um die Uhr klingelten. Diese lange Bearbeitungsdauer und auch die späte Gründung des Nationalfonds an sich habe bei den AntragstellerInnen zu gemischten Gefühlen und oft auch Aufregung geführt (vgl. Int.6, S.2, Z.48-53). Durch die fehlende Organisationsstruktur erfolgte die Arbeit planlos. (vgl. Int.6, S.1, Z.31-33). Die Mitarbeiterin beschreibt die Zustände zu Beginn als chaotisch. 1995 gab es noch kein Internet, es gab generell nur wenige Computer im Büro, der Hauptteil der Arbeit wurde manuell erledigt (vgl. Int.6, S.2-3, Z.71-82). Auch die Einrichtung einer Datenbank und die Dateneingabe waren eine sehr große Herausforderung (vgl. Janistyn-Novák, S.6, Z.192-194 und Int.1, S.3, Z.78-81). Der Arbeitsaufwand war enorm, "[...] es wurde wahnsinnig viel auch händisch gemacht [...]." (Int.6, S.3, Z.79) Die Eingabe eines Datensatzes in das Computersystem dauerte bis zu einer Stunde (vgl. Int.6, S.2-3, Z.80-82).

Bevor die ersten Zahlungen getätigt werden konnten, mussten die potentiellen Antragstellerlnnen durch öffentliche Kundmachungen informiert werden. Besprechungen mit den Opferverbänden hinsichtlich der Kriterien der Antragstellung wurden geführt. Es wurde festgelegt, dass die Anträge nach Alter der Antragstellerlnnen bearbeitet werden sollten, damit die Auszahlungen prioritär an die ältesten Opfer erfolgen konnten (vgl. Janistyn-Novák, S.6, Z.198-207). 
Antragsformulare wurden in zwei Sprachen erstellt und Überlegungen wurden angestellt, welche Belege verlangt werden sollten, um eine Zahlung zu ermöglichen (vgl. Janistyn-Novák, S.6, Z.212-214).

Dr. Janistyn-Novák zufolge sind frühere Entschädigungsmaßnahmen, wie durch die Rückstellungskommission und die Opferfürsorge, oft an fehlenden Belegen gescheitert. Es wurden Belege von den Menschen verlangt, die auf ihrer Flucht oftmals alles verloren hatten (vgl. Janistyn-Novák, S.7, Z.217-220). Die Herausforderung im Nationalfonds war, Lösungen für fehlende Belege zu finden, um im Sinne der AntragstellerInnen entscheiden zu können. Viele Quellen mussten herangezogen werden, unter anderem Meldeevidenzen, um doch Nachweise zu finden (vgl. Janistyn-Novák, S.7, Z.224-234).

Auch für Frau Mag. Lessing war die Administration des Fonds eine große Herausforderung. Für sie war es essentiell, genügend Mitarbeiterlnnen einstellen zu können, die die Recherche für die AntragstellerInnen übernehmen und für die Leute da sein würden,

[...] weil diese Menschen haben keine Dokumente. Mein Papa ist mit 16 geflohen, der hat keinen Koffer mitgenommen mit allen Dokumenten [...] das war die Herausforderung. (Lessing, S.2, Z.60-63)

Eine Kooperation mit sämtlichen Archiven war ausschlaggebend für Frau Mag. Lessing. Es war ihr ein Anliegen, dass die Recherchearbeiten von MitarbeiterInnen übernommen wurden. Niemand sollte aufgrund fehlender Nachweisdokumente abgewiesen werden. (vgl. Lessing, S.2, Z.63-69).

Die Beschaffung der Nachweisdokumente der Verfolgung stellte auch für Dr. Meissner eine Herausforderung dar. Die Zusammenarbeit mit Archiven und anderen Organisationen war dafür essentiell, barg aber auch Schwierigkeiten.

[...] die Schwierigkeit war, dass diese Institutionen und ihre Archive eigentlich als wissenschaftliche Archive konzipiert sind und auch nicht gedacht waren, 
dass die tausende Anfragen im Monat kriegen und das war dann nicht nur unsere Herausforderung, sondern die Herausforderung für all diese Institutionen, das zu gewährleisten, mit wenig Personal [...], es waren ganz schwierige Verhandlungen und die Leute waren wirklich überfordert ja, haben aber das Beste geleistet, weil ja viele der Leute, die verfolgt wurden, im Laufe ihrer Flucht oder schon vorher die Dokumente nicht mitnehmen konnten [...] ganz große Herausforderung, das Dokumentenmaterial für die Leute sehr schnell zu erwirken und da ist wirklich Großartiges geleistet worden [...]. (Meissner, S.8, Z.263-273)

Eines der Erfolgsrezepte des Nationalfonds war für Frau Dr. Janistyn-Novák der Konsens mit der Politik im Sinne einer möglichst unbürokratischen Herangehensweise, da vollständige Nachweise nicht immer gelingen konnten (vgl. Janistyn-Novák, S.7, Z.231-234). Eine weitere Herausforderung war nicht nur die Arbeitsbelastung hinsichtlich der langen Arbeitszeiten, sondern auch die psychische Belastung für die MitarbeiterInnen. Täglich mit den Erinnerungen und auch den Reaktionen und Empfindungen der Antragstellerlnnen, wenn sie ihre Erinnerungen teilten, konfrontiert zu sein, war oft nicht einfach. Auch mit möglichen Retraumatisierungen der AntragstellerInnen musste man rechnen. Sowohl diese Hingabe als auch die Belastung habe die Mitarbeiterlnnen laut Dr. Janistyn-Novák sehr verbunden (vgl. Janistyn-Novák, S.7, Z.251-258). Retraumatisierungen der Antragstellerlnnen waren nicht selten. Sie erinnert sich, dass etliche AntragstellerInnen im Nationalfonds zum ersten Mal ihre Lebensgeschichte erzählt haben. Viele wollten nicht, dass die eigenen Familien von ihrem Schicksal erfuhren. Ein Antragsteller hätte seine Angst vor Behörden nicht überwinden können und das Parlamentsgebäude nicht betreten, sondern eine Freundin geschickt, die seine Unterlagen abgegeben hat (vgl. Janistyn-Novák, S.7-8, Z.258-264).

Trotz dieser emotionalen Belastungen sind die Antragstellerlnnen dennoch an den Nationalfonds herangetreten und haben den MitarbeiterInnen ihre Erinnerungen anvertraut. Durch den Nationalfonds hat die Anerkennung als Opfer des 
Nationalsozialismus ein persönliches Gesicht bekommen. "Das waren nicht Zahlen, sondern das waren einzelne Menschen, die Fürchterliches erlebt haben." (JanistynNovák, S.8., Z.286-287)

Der Umgang mit den emotionalen Reaktionen der Antragstellerlnnen auf die Gespräche mit den MitarbeiterInnen, die keine PsychologInnen waren, war auch für Frau Dr. Meissner eine Herausforderung, die nur mit Engagement und Hingabe gelingen konnte. Sie erzählt, dass sie mit den unterschiedlichen Emotionen der AntragstellerInnen konfrontiert waren. Die Emotionen reichten von Trauer bis hin zu Wut Österreich gegenüber (vgl. Meissner, S.6, Z.214-220). Sie empfingen täglich zwischen 30 und 60 Antragstellerlnnen (vgl. Meissner, S.7, Z.225-226). Besonders betroffen machte die intensive Konfrontation mit den Erzählungen der Menschen über ihre Schicksale:

[...] wenn man dann [...] hört, dass ein Antragsteller plötzlich erzählt und wirklich weinend niederbricht und erzählt, [...] er war dabei wie [...] seinem Bruder der Kopf gespalten wurde oder eine Dame, die bei mir war und mir unter Tränen erzählt hat, sie musste als 15-jähriges Mädchen die Zähne und Haare von den, in den Gaskammern Ermordeten, sortieren [...]. (Meissner, S.6-7, Z.220-224)

Der sensible Umgang mit den Antragstellerlnnen zählte für Frau Mag. Lessing ebenfalls zu den Herausforderungen der ersten Stunde.

[...] wir haben nicht gewusst, was uns erwartet und plötzlich war unser Büro überlaufen von Menschen und nicht, weil wir das Gefühl hatten, dass sie jetzt sagen, ha, jetzt kriegen wir viel Geld, sie wollten reden und das war wirklich, glaube ich, unsere größte Herausforderung am Anfang, zuzuhören, sich selber aber auch natürlich schützen, weil sie haben uns an der Hand genommen und [...] ihre Erinnerungen bei uns abgeladen, Wunden sind aufgegangen, 
Menschen haben geweint, [..] es war ein Gemisch an unglaublichen Gefühlen, das war, glaube ich, die größte Schwierigkeit am Anfang. (Lessing, S.2, Z.4552)

Laut einer Mitarbeiterin war die erste Herausforderung des Nationalfonds, ein Bewusstsein für die Thematik in der Gesellschaft zu schaffen. Diese Bewusstseinsbildung in bestimmten Bevölkerungskreisen hatte bereits vorher teilweise durch die Waldheim-Affäre begonnen (vgl. Int.3, S.1-2, Z.34-39).

Und ich glaube, die große Mehrheit in der Bevölkerung wollte das Thema immer abgeschlossen sehen und ich glaube, das war die große Herausforderung, da ein anderes Bewusstsein langsam einzuführen, was sicher nicht für alle gelungen ist, aber doch, doch in einem beachtlichen Maß, denke ich. (Int.3, S.2, Z.39-42)

Auch eine andere Mitarbeiterin sah die erste Herausforderung ebenfalls in der Bewusstseinsbildung und Bekanntmachung, dies bedeutete auch, gegen Widerstände anzugehen. Die zweite Herausforderung lag ihrer Meinung nach im Zugang zu den potentiellen Antragstellerlnnen. Es musste ein Weg gefunden werden, die Menschen zu erreichen und empathisch auf sie zuzugehen (vgl. Int.4, S.1, Z.28-32). Als weitere Herausforderung sah sie auch den damals gewünschten raschen Beginn der Entschädigungszahlungen an die Opfer, von denen so viele wie möglich erreicht werden sollten (vgl. Int.4, S.1, Z.36-37).

Frau Mag. Lessing sah die erste Herausforderung darin, einen Zugang zu den Menschen zu finden. Ihr Vater habe ihre Tätigkeit zuerst abgelehnt, er wollte nicht, dass sie sich mit dieser Thematik beschäftigt und er selbst habe kaum darüber gesprochen. Frau Mag. Lessing erzählt, dass sie über seine Erwartungshaltung ihrer Aufgabe gegenüber mit inm zu sprechen versuchte: 
[...] er war ja so ein Schweiger und hat das auch nie besprochen mit uns [...], aber ich habe gewusst, ich möchte das machen und wie er mir gesagt hat, [...], kannst du mir meine Kindheit zurückgeben oder kannst du mir meine Mutter aus Auschwitz zurückgeben, war mir klar, das wird [..] der größte Schritt sein am Anfang, dass wir [...] 50 Jahre nach Kriegsende überhaupt erst auf so viele Menschen zugehen können, wie erklären wir ihnen, dass es ein anderes Land jetzt ist, dass das Schweigen überwunden werden kann? (Lessing, S.1, Z.29-35)

Eine weitere Herausforderung war die Menge an Menschen, die in den Nationalfonds kamen und über ihre Geschichte sprechen wollten. Frau Mag. Lessing erzählt, dass auch aufgrund der Waldheim-Affäre und der Rede von Bundeskanzler Vranitzky das kollektive Schweigen bereits gebrochen war (vgl. Lessing, S.1-2, Z.36-41).

Den Nationalfonds, nur auf Grundlage des Nationalfondsgesetzes, von Grund auf aufzubauen, war für Frau Dr. Meissner die größte Herausforderung. Es musste eine Struktur geschaffen werden, ein Büro gefunden werden, Antragsformulare erstellt werden und die Anspruchsberechtigten gefunden werden (vgl. Meissner, S.4, Z.113124). Mit dem Gesetz wurde zwar die Grundlage für den Nationalfonds geschaffen, die Herausforderung lag dann aber in der Exekution des Gesetzes und der Umsetzung und Gestaltungsmöglichkeiten in organisatorischer Hinsicht (vgl. Meissner, S.4, Z.124-131). Der Parteienverkehr musste organisiert werden. Frau Dr. Meissner erzählt, dass versucht wurde, hier eine Struktur zu finden. Die AntragstellerInnen wurden vormittags empfangen und nachmittags waren die Mitarbeiterlnnen für die einlangenden Telefonate zuständig. Der Arbeitsdruck war enorm, es fanden alle vier bis sechs Wochen Sitzungen des Komitees statt, in denen ca. 800-1000 Anträge entschieden werden sollten (vgl. Meissner, S.7, Z.230-238). Eine Mitarbeiterin erzählt ebenfalls, dass pro Komitee-Sitzung und Umlauf [schriftliches Beschlussverfahren ohne Zusammenkunft des Komitees, Anm. d. Verf.] um die 1000 Anträge entschieden wurden. Der Arbeitsaufwand war sehr hoch, 
ebenso die Bearbeitungsdauer der Erfassung der Anträge im Computersystem (vgl. Int.1, S.2, Z.59-65).

Die Herausforderung, die potentiellen Antragstellerlnnen weltweit zu finden, wurde zur Hauptaufgabe von Frau Mag. Lessing, wie Frau Dr. Meissner schildert.

Eben nicht nur über Botschaften, Zeitungen, Inserate, sondern auch durch ihre Reisen in die ganze Welt und Vorträge, einfach den Nationalfonds, vor allem im Ausland aber auch im Inland publik zu machen, dass die Leute überhaupt von dieser Maßnahme erfahren. (Meissner, S.7, Z.249-252)

Dr. Meissner fasst zusammen, dass die geringen zeitlichen, personellen und auch technischen Ressourcen eine Herausforderung darstellten (vgl. Meissner, S.8, Z.276281). Die langen Arbeitszeiten wurden in Kauf genommen, damit die AntragstellerInnen aus dem Ausland trotz der Zeitverschiebung mit dem Nationalfonds Kontakt aufnehmen konnten. Ohne dieses persönliche Engagement der Mitarbeiterlnnen wäre das nicht möglich gewesen (vgl. Meissner, S.8, Z.285289).

\section{Herausforderungen nach der Umstrukturierung 2019}

Für Frau Dr. Janistyn-Novák besteht eine Herausforderung für die Zukunft des Nationalfonds darin, weiterhin engagierte Mitarbeiterlnnen sowie geeignete NachfolgerInnen für die Geschäftsleitung zu gewinnen (vgl. Janistyn-Novák, S.18, Z.654-659).

Frau Dr. Uhl sieht die zukünftige Positionierung des Nationalfonds mit seinen neuen Aufgaben im Feld der Erinnerungskultur als Herausforderung (vgl. Uhl, S.8, Z.282284)

Für eine Mitarbeiterin besteht die Herausforderung für die zukünftige Arbeit des Nationalfonds darin, „[...] all diesen Anforderungen gerecht zu werden und das [...] umsetzen zu können." (Int.1, S.6, Z.189-190) Ein Mitarbeiter vermutet, dass mit der 
letzten Gesetzesnovelle eine gute Grundlage für die zukünftige Arbeit des Nationalfonds geschaffen wurde, diese Grundlage aber immer auch politischen Rahmenbedingungen unterliegen würde. Es sei ein Experiment, dessen Auswirkungen noch nicht ersichtlich seien (vgl. Int.2, S.5, Z.164-170).

Die Neugestaltung von Arbeitsabläufen und Vorgehensweisen aufgrund der zukünftigen Aufgaben nach der Umstrukturierung sei wichtig, erläutert eine andere Mitarbeiterin (vgl. Int.4, S.2, Z.48-51). Eine weitere Herausforderung in der Zukunft sieht sie in der Umgestaltung der Aufgaben. Es brauche in Zukunft mehr Kooperationen mit Organisationen und mehr Vermittlungsarbeit (vgl. Int.4, S.3, Z.8894). Sie sieht es auch als Herausforderung an, den Nationalfonds in Zukunft mit der neuen Struktur zu positionieren. Es bestünde jedoch das Risiko, dass dies nicht gelingen könnte, wenn der Nationalfonds nicht aktiver die Zusammenarbeit mit anderen Organisationen suchen würde (vgl. Int.4, S.4, Z.126-131).

Auch für eine andere Mitarbeiterin stellt es eine Herausforderung für die Zukunft dar, vermehrt an die Öffentlichkeit zu treten. Für sie wäre es wichtig, "[...] öffentlich auftreten und wahrgenommen $\mathrm{zu}$ werden, auch als eine Stelle, die gegen Rassismus, Antisemitismus, für Menschenrecht eintritt." (Int.5, S.2, Z.59-61) Die Ausweitung der Öffentlichkeitsarbeit sieht auch eine weitere Mitarbeiterin als Herausforderung. Ihrer Meinung nach hat der Nationalfonds bisher hier keine aktive Rolle gespielt (vgl. Int.6, S.4, Z.112-118).

Politische Änderungen wirken sich nach Meinung einer Mitarbeiterin auch auf den Nationalfonds aus (vgl. Int.6, S.3, Z.100-103). Für sie wäre es wichtig, Netzwerke aufzubauen (vgl. Int.6, S.3, Z.107-108).

Für Frau Mag. Lessing ist es eine Herausforderung und ein gesellschaftlicher Auftrag, in Zukunft neue Wege zu beschreiten. Man müsse hinterfragen, warum trotz der jahrelangen Vermittlungsarbeit die Jugend dennoch nicht genug erreicht werden konnte (vgl. Lessing, S.7, Z.247-256).

Die zukünftige Herausforderung läge in der umfassenden Aufarbeitung des Archivs für die Wissenschaft und die Nachwelt, berichtet Frau Dr. Meissner (vgl. Meissner, 
S.10, Z.344-347). Ein Fokus werde auch auf der inhaltlichen Gestaltung und dem Ausbau der zukünftigen Aufgaben des Nationalfonds liegen (vgl. Meissner, S.13, Z.475-477).

\subsubsection{Kategorie 5: Zukunftsperspektiven für den Nationalfonds}

Aufgrund der Fragestellungen wurde diese Kategorie in drei Subkategorien unterteilt. Die erste Subkategorie enthält Aussagen zu den zukünftigen Aufgaben, die der Nationalfonds wahrnehmen wird bzw. wahrnehmen kann. Die zweite und dritte Subkategorie beleuchtet die Meinungen der befragten Personen zu den zukünftigen Chancen und Risiken für den Nationalfonds.

\section{zukünftige Aufgaben des Nationalfonds}

Frau Dr. Janistyn-Novák sieht die Wissensvermittlung auch in der Zukunft als zentrale Aufgabe des Nationalfonds. Vor allem die Publikationen des Nationalfonds zu den Lebensgeschichten der Opfer würden zu einer Bewusstseinsbildung und Mahnung beitragen (vgl. Janistyn-Novák, S.9, Z.317-321). Die Lebensgeschichten würden einen niederschwelligen Ansatz für die Auseinandersetzung mit den Geschehnissen des Nationalsozialismus darstellen, deren Wirkung man sich kaum entziehen könne (vgl. Janistyn-Novák, S.11, Z.369-379). Der Nationalfonds könne die vielen, gesammelten Berichte der ZeitzeugInnen an Schulen und an Jugendliche weitergeben und damit die Rolle der bald fehlenden Zeitzeuglnnen ein Stück übernehmen (vgl. Janistyn-Novák, S.12, Z.426-434). Die gesammelten Materialien im Archiv des Nationalfonds würden umfassende Möglichkeiten bieten, die Schicksale und Geschichten der Opfer lebendig zu halten (vgl. Janistyn-Novák, S.11, Z.382386). Durch die Menge an Material im Archiv des Nationalfonds wird es auch laut Frau Mag. Lessing möglich sein, weiterhin mit den Geschichten der Überlebenden zu arbeiten, selbst wenn sie nicht mehr am Leben sind (vgl. Lessing, S.13, Z.466-470).

Das Parlament würde sich derzeit ebenfalls wieder verstärkt mit dem Thema Antisemitismus beschäftigen, erzählt Frau Dr. Janistyn-Novák. Hier könnten in Zukunft gemeinsam mit dem Nationalfonds weitere Kooperationen, auch mit anderen Organisationen, entstehen (vgl. Janistyn-Novák, S.9, Z.321-328). „[..] mitzuwirken und [...] entsprechende Forschungen [...] zu unterstützen, das glaube ich, ist 
tatsächlich für die Zukunft nach wie vor eine ganz zentrale Aufgabe und [...] auf diesem Gebiet ist der Nationalfonds ein Kompetenzzentrum der Republik." (JanistynNovák, S.9, Z.328-331) Die Vernetzung mit anderen Organisationen sei essentiell, um gemeinsame Vorgehensweisen abstimmen und Widersprüche oder Verdoppelungen vermeiden zu können (vgl. Janistyn-Novák, S.9, Z.333-338).

Die weiterführende Bekanntmachung von entzogenem Eigentum in Datenbanken für eine mögliche Restitution könnte durch den Nationalfonds erfolgen. Dr. JanistynNovák nennt als Beispiel die Restitution von Büchern, deren ideeller Wert für die Nachkommen oftmals von großer Bedeutung sei (vgl. Janistyn-Novák, S.14, Z.496504).

Laut Frau Dr. Janistyn-Novák wird die Zukunft auch in den Vernetzungen mit anderen Organisationen liegen. Es gelte eine gemeinsame Strategie zu verfolgen und Widersprüche zu vermeiden (vgl. Janistyn-Novák, S.10, Z.332-338). Der Nationalfonds erfülle vor dem Hintergrund des Nationalsozialismus und des Holocaust, der einen Bruch in der Zivilisationsgeschichte darstellt, eine Aufgabe, die laut Frau Dr. Janistyn-Novák nie erledigt sein wird (vgl. Janistyn-Novák, S.12, Z.426434). Die Gewichtung der wissenschaftlichen Disziplinen könnte sich in Zukunft verlagern. Zu Beginn der Aufarbeitung der Geschichte spielten ZeithistorikerInnen eine zentrale Rolle. Jetzt wäre es für Frau Dr. Janistyn-Novák auch denkbar, dass es zu einer Erweiterung der Forschungsfragen durch Kooperationen verschiedener wissenschaftlicher Disziplinen kommen könnte (vgl. Janistyn-Novák, S.15, Z.523530). Sie sieht die Zukunft des Nationalfonds auch in der aktiven wissenschaftlichen Aufarbeitung des Archivs und der Veröffentlichung der Ergebnisse, sowie in der Vermittlungsarbeit (vgl. Janistyn-Novák, S.15, Z.535-540).

Es müsse ein Bezug zur Gegenwart hergestellt werden und die Aufgaben des Fonds sollten mit Fragen der Menschenrechte und der Menschenwürde verknüpft werden. Dr. Janistyn-Novák würde sich wünschen, dass der Nationalfonds hier auch in Zukunft eine führende Rolle einnimmt (vgl. Janistyn-Novák, S.15, Z.542-550). Ein Gegenwartsbezug sollte vor allem auch in Hinblick auf die Thematik der gesellschaftlichen Ausgrenzung, die nach wie vor aktuell sei, hergestellt werden, betont Frau Dr. Janistyn-Novák (vgl. Janistyn-Novák, S.13, Z.470-474). 
Frau Mag. Maschke betont die derzeit bereits bestehenden, guten Kooperationen des Nationalfonds mit anderen Playern in diesem Tätigkeitsfeld wie mit ,_erinnern.at_, dem Holocaust Education Institute des Bildungsministeriums. Sie würde sich wünschen, dass der Nationalfonds als Organisation, die bei der Parlamentsdirektion angesiedelt ist, die Koordinierungsstelle für das staatliche Gedenken und Erinnern Österreichs wird (vgl. Maschke, S.9, Z.321-331). Für sie ist der Nationalfonds eine der wesentlichen Institutionen im Handlungsfeld (vgl. Maschke, S.3, Z.108-110). Sie hält auch fest, dass Österreich ein Holocaust-Museum benötigen würde, der Nationalfonds könnte in Zukunft auch hier als Koordinierungsstelle fungieren (vgl. Maschke, S.10, Z.332-334).

Für Frau Dr. Uhl ist es fraglich, welchen Platz der Nationalfonds in Zukunft einnehmen kann. Auch sie merkt an, dass in der österreichischen Erinnerungslandschaft ein Holocaust Museum noch fehlen würde. Diese Lücke wird jedoch nach Dr. Uhl nicht so schnell gefüllt werden (vgl. Uhl, S.7, Z.230-233). Die Positionierung des Nationalfonds in Zukunft stelle eine Herausforderung dar ( $v g l$. Uhl, S.8, Z.282-284). Man müsse eine Lücke in dem Feld finden, die durch den Nationalfonds geschlossen werden könnte. (vgl. Uhl, S.8, Z.288-289).

Eine Mitarbeiterin würde sich wünschen, dass der Nationalfonds auch in Zukunft noch beim Parlament angesiedelt ist und größere Anerkennung erfährt (vgl. Int.1, S.9, Z.310-312). Ein anderer Mitarbeiter gibt zu bedenken, dass mit gleichbleibenden Mitteln und personellen Ressourcen nicht mehr als bisher vom Nationalfonds geleistet werden könne (vgl. Int.2, S. 16, Z.565-570). Der Nationalfonds sei auch keine Akademie der Wissenschaften. Es könnten zwar Konzepte erarbeitet werden, aber das Wissen im Nationalfonds beruhe zum großen Teil auf jahrelangen Erfahrungswerten und Erkenntnissen aus Selbststudien (vgl. Int.2, S.16, Z.582-587). Für inn bleibt offen, ob aufgrund der vorhandenen Mittel dennoch neue Ideen umgesetzt werden können, ohne die Mitarbeiterlnnen zu sehr zu belasten (vgl. Int.2, S.16-17, Z.591-600). Für den befragten Mitarbeiter wäre es auch vorstellbar, dass es den Nationalfonds in 20 Jahren nicht mehr geben könnte oder er vielleicht unter anderem Namen mit anderen Aufgaben weiter existieren würde (vgl. Int.2, S.18-19, Z.660-669). 
Eine Herausforderung für den Nationalfonds in Zukunft sei die Wissensvermittlung an Jugendliche, betont eine Mitarbeiterin. Auch sie nennt die Notwendigkeit neuer Anknüpfungspunkte und eines Gegenwartsbezugs (vgl. Int.3, S.13, Z.457-465).

Deswegen sehe ich da auch eine [...] Zukunft für den Nationalfonds, ja, weil ich glaube, dass das, was wir hier erlebt haben, diese vielen, vielen Kontakte mit Antragsstellern, das ist [...] ein Privileg und etwas, was aber auch ein Auslaufmodell ist [...], aber auf eine mittelbare Art werden wir selber zu Zeitzeugen [...] und ich glaube, das ist schon ein bisschen eine Verpflichtung, dass man [...] das verstärken könnte in Zukunft. (Int.3, S.13-14, Z.470-476)

Wünschenswert wäre der Mitarbeiterin zufolge eine Einrichtung des Nationalfonds als dauerhafte Institution im Parlament, " [...] der sozusagen ein bisschen dieses wache Auge auf politische Entwicklungen [...] in Erinnerung hält [...]" (Int.3, S.17, Z.596-597) Erinnerungs- und Gedenkarbeit mit Bezug zur Gegenwart, zu heutigem Antisemitismus und Rassismus, sieht auch eine weitere Mitarbeiterin als wünschenswerte, zukünftige Aufgabe des Nationalfonds (vgl. Int.4, S.1-2, Z.37-43). Für die Zukunft wäre der Mitarbeiterin auch wichtig, dass der Nationalfonds sich tagespolitisch zu Wort melden könne (vgl. Int.4, S.8, Z.292-295). Es sei ungewiss, ob der Nationalfonds eine Zukunft haben könne, es gäbe Gerüchte im politischen Umfeld, eine Zentralisierung anzustreben. Sie hege Zweifel, ob der Nationalfonds für die Zukunft gut aufgestellt sei (vgl. Int.4, S.9, Z.299-308).

Für eine weitere Mitarbeiterin liegt die Zukunft ebenso in der Vermittlungsarbeit und in der Kooperation und Vernetzung mit anderen Organisationen. Für sie ist eine feste Verankerung in der österreichischen Erinnerungslandschaft denkbar (vgl. Int.5, S.8, Z.286-293). Zukünftig mehr Vernetzungen mit anderen Organisationen herzustellen, ist auch für eine andere Mitarbeiterin von großer Bedeutung (vgl. Int.6, S.5, Z.177). Dennoch sei die Zukunft des Nationalfonds in ihren Augen ungewiss (vgl. Int.6, S.11, Z.384-385). 
Vermittlungsarbeit und Vernetzung mit Organisationen sind auch für Frau Mag. Lessing ein zentrales Thema für die zukünttige Arbeit (vgl. Lessing, S.6-7, Z.215221). Die Zukunft des Nationalfonds liegt laut Frau Mag. Lessing auch in der weiteren Aufarbeitung der gesammelten Materialien. Man müsse sich überlegen, wie man die Zeitzeuglnnen weiterleben lassen könne. Eine Möglichkeit hier würde durch ZeitzeugInnen zweiter Generation bestehen. Sie selbst gehe oft an Schulen und erzähle von der Geschichte ihres Vaters, es sei zwar nicht mehr seine direkte Geschichte, aber sie könne erzählen, "[...] was es geheißen hat, mit so einem Vater aufzuwachsen." (Lessing, S.14, Z.478)

Die Kunstrestitution, vor allem auch im privaten Bereich, wird laut Frau Mag. Lessing ebenfalls ein Betätigungsfeld des Nationalfonds bleiben. Sie könnte sich vorstellen, dass durch die Erforschung des Archivs auch eventuell wieder mehr HistorikerInnen beschäftigt sein werden. Sie hofft, dass der Nationalfonds auch weiterhin beim Nationalrat eingerichtet sein wird (vgl. Lessing, S.16, Z.558-566). "[...] im Nationalrat angesiedelt $\mathrm{zu}$ sein mit diesem Thema ist wichtig und daher sehe ich den Nationalfonds weiterhin genau da, wo wir sind, mit genau diesen Aufgaben." (Lessing, S.16, Z.581-583)

Frau Dr. Meissner sieht die zukünftigen Aufgabengebiete ebenfalls in der Aufarbeitung des Archivs. Durch die Digitalisierung des Archivs würden sich neue Möglichkeiten bieten. Sie sieht den Nationalfonds in Zukunft verstärkt als Servicestelle für Nachfolgegenerationen der Opfer des Nationalsozialismus. Der Fokus werde auf Wissenschaft, Aufarbeitung und Vermittlung liegen (vgl. Meissner, S.9-10, Z.329-344). In 20 Jahren könnte der Nationalfonds in einem Verbund von anderen Organisationen, die sich mit Gedenken, Vermittlung und Wissenschaft beschäftigen, verankert sein. Es wäre für sie vorstellbar, dass es in Österreich eine große Institution geben könnte, wie es bereits in anderen Ländern der Fall ist, wo all diese Themen verknüpft werden könnten (vgl. Meissner, S.27, Z.962-974). Sie ist auch der Meinung, dass der Nationalfonds in Zukunft eine zentrale Ansprechstelle für die zukünftigen Generationen sein wird, in der "[...] Menschliches und Wissenschaftliches zusammenfließt." (Meissner, S.27, Z.979) Durch die aufgezeichneten Erinnerungen der Überlebenden könne Geschichte auch in Zukunft weiterhin erlebbar gemacht werden (vgl. Meissner, S.23-24, Z.847-859). 
Und natürlich auch durch die Tätigkeit von Mitarbeitern und Mitarbeiterinnen, die dieses Wissen an Schulen tragen und bereit sind für Vorträge an Universitäten, an Interessierte, an Volkshochschulen. Also ich glaube, auch auf der persönlichen Ebene, nicht nur durch die Verbreitung von Publikationen, kann der Nationalfonds wahnsinnig viel noch beitragen und wird das vermehrt in Zukunft auch tun, als einer seiner Aufgaben in der Vermittlungstätigkeit [...] (Meissner, S.24-25, Z.882-887)

\section{Chancen}

Die Chance, dass der Nationalfonds weiterhin in der österreichischen Gedenklandschaft so etabliert bleibt, empfindet eine Mitarbeiterin als sehr groß. Dafür sei die Umstrukturierung notwendig gewesen (vgl. Int.1, S.6, Z.194-196).

Das Alleinstellungsmerkmal des Nationalfonds, beim Parlament angesiedelt zu sein, sei eine Chance für die Zukunft, erklärt eine weitere Mitarbeiterin (vgl. Int.3, S.9, Z.305-306). Diese Chance liege in "[...] einer klareren und stärkeren Positionierung in der Öffentlichkeit [...]" (Int.3, S.8, Z.294) Sie hebt hervor, dass der Nationalfonds auch zukünftig über dem tagespolitischen Geschehen stehen und vermehrt auf politische Grundhaltungen achten sollte (vgl. Int.3, S.8-9, Z.292-305). Einer anderen Mitarbeiterin zufolge liegt eine Chance des Weiterbestehens des Nationalfonds in der Klärung der internen Entscheidungskompetenzen (vgl. Int.5, S.4, Z.130-131). Sie bezieht sich damit auf die neue Struktur des Nationalfonds. Durch eine zukünftig transparente Informationsweitergabe von der Führungsebene könnten auch bereichsübergreifende Projekte verwirklicht werden (vgl. Int. 5, S.4, Z. 136-138). Eine Chance für den Nationalfonds liege in der Zusammenarbeit mit Organisationen, betont eine andere Mitarbeiterin (vgl. Int.6, S.5, Z.170-172).

Als Chance sieht Frau Mag. Lessing die Tatsache, „[...] dass wir sicher ein unglaubliches institutionelles Wissen haben [...]." (Lessing, S.7, Z.226-227) Für sie stellt auch das Archiv eine Chance dar. 
Dieses Archiv, [...] die Registratur, die wir haben, ist eine Riesen-Chance, weil ich glaube, das gibt es kaum weltweit, dass du nicht nur ein jüdisches Archiv hast, sondern eben alle Opfergruppen hast, dass wir über 24 Jahre Kontakt hatten zu den Überlebenden, die Geschichte ihrer Eltern und Großeltern aufgezeichnet haben, aber teilweise jetzt schon mit der Enkelgeneration in Kontakt sind, das heißt, unsere Registratur beinhaltet die Jahre 1870 bis 2019 , also das sind fünf, sechs Generationen, [...] das gibt es sonst nirgendwo, das ist eine Riesen-Chance. (Lessing, S.7, Z.230-236)

Frau Dr. Meissner sieht die Chance für eine Zukunft des Nationalfonds in der gesellschaftspolitischen Aktualität der Thematik. Auch nach fast 25-jährigem Bestehen des Nationalfonds sei die Arbeit noch lange nicht beendet, darin liegt für sie die Chance für ein Weiterbestehen (vgl. Meissner, S.18, Z.637-644).

Es ist eine Chance, wechselweise kann man sagen, für die Gesellschaft, noch mehr zu erfahren von den Sachen, die wir gerade aufarbeiten. Es ist eine wahnsinnige Chance für die Wissenschaft, dann später auf das zurückgreifen zu können [...] und es ist eine wahnsinnige Chance für die zweite und dritte Generation auch mit uns noch zu kommunizieren. Etwas über ihre, durch unsere Recherchen, über ihre Familien zu erfahren, [...] also dieses Wechselspiel ist für den Nationalfonds [eine] wahnsinnige Chance in Zukunft weiter zu bestehen und tolle Arbeit zu leisten, [...] mit den letzten noch lebenden Antragstellern, mit Wissenschaftlern, mit Schülerinnen und Schülern [...] (Meissner, S.18, Z.644-652)

In Kooperationen mit Organisationen und Vereinen besteht Frau Dr. Meissner zufolge ebenfalls eine Chance für die Zukunft (vgl. Meissner, S.19, Z.666-676). 


\section{Risiken}

Einem Mitarbeiter zufolge würde ein Scheitern der Organisationsreform ein Risiko für das Fortbestehen des Nationalfonds darstellen, in dem Fall wäre nicht nur der Nationalfonds, sondern auch die Geschäftsführung und die Generalsekretärin sehr gefährdet. Man würde dann zu einem politischen Spielball werden (vgl. Int.2, S.6-7, Z.215-229). Eine andere Mitarbeiterin merkt an, dass die neue Struktur und die neu eingeführte zusätzliche Hierarchie Verzögerungen in Entscheidungsfindungen begünstigen könnten. Dies würde für sie ein Risiko darstellen (vgl. Int.5, S.4, Z.125129).

Die Verrohung der Gesellschaft bezeichnet Frau Mag. Lessing als Risiko. Sie würde das jedoch nicht als Gefahr für den Fortbestand des Nationalfonds sehen, sondern vielmehr als „[...] eine Aufforderung an den Nationalfonds, dass wir weitermachen und neue Wege beschreiten [...]." (Lessing, S.7, Z.252). Die Zukunft des Nationalfonds sei auch vom politischen Willen abhängig, wie Frau Dr. Meissner erklärt. Es könne durchaus der Eindruck entstehen, dass sich zu viele Organisationen mit der gleichen Thematik beschäftigen. Sie sehe hier dennoch eine Chance in der Zusammenarbeit und Vernetzung, da die Schwerpunkte der verschiedenen Institutionen doch in unterschiedlichen Bereichen liegen würden (vgl. Meissner, S.18-19, Z.654-666).

\subsubsection{Kategorie 6: Meilensteine in der Erinnerungskultur}

In Bezug zur erarbeiteten theoretischen Erläuterung einiger Meilensteine der österreichischen Erinnerungskultur wurde auch in den Interviews nach Meilensteinen gefragt. Besonderes Augenmerk lag auf der Serie ,Holocaust', daher wurden die Aussagen hierzu in einer eigenen Subkategorie erfasst. Als zweite, induktive Subkategorie ergab sich in der Auswertung der Aussagen ein weiterer Meilenstein, nämlich die Gründung des Nationalfonds.

Frau Dr. Janistyn-Novák erzählt, dass die Erklärung des damaligen Bundeskanzlers Dr. Vranitzky in Israel viel bewegt hat (vgl. Janistyn-Novák, S.1, Z.48-53). Die Waldheim-Affäre und die Reden von Bundeskanzler Vranitzky stellten auch Frau Mag. Lessing zufolge einen Durchbruch dar (vgl. Lessing, S.1-2, Z.36-38). Durch 
diese Meilensteine wurde die beginnende Umkehr der Opferthese auch laut Frau Dr. Meissner möglich. Die Veränderung der Sichtweise habe eine andere Gedenk- und Erinnerungskultur ermöglicht (vgl. Meissner, S.21, Z.757-763).

\section{Die Serie , Holocaust}

Für Frau Dr. Uhl ist die Serie 'Holocaust' sozusagen ein Klassiker unter den Meilensteinen der Erinnerungskultur (vgl. Uhl, S.3, Z.99). Die Serie habe den "[...] Perspektivenwechsel auf die Geschichte der Opfer ganz entschieden angestoßen." (Uhl, S.3, Z.102)

Eine befragte Mitarbeiterin hat die Serie aufgrund ihres damals zu jungen Alters nicht gesehen (vgl. Int.1, S.7, Z.245). "Mir war nicht einmal bewusst, dass ich das damals irgendwo gesehen hätte oder dass es irgendwo gelaufen ist." (Int.1, S.7, Z.251-253)

Ein anderer Mitarbeiter erinnert sich, damals eine Sammlung von Zeitungsausschnitten zu der Serie angelegt zu haben. An die Serie selbst erinnere er sich jedoch kaum mehr (vgl. Int.2, S.10-11, Z.369-372). Die Auswirkungen der Serie formuliert er wie folgt: "Ich könnte mir vorstellen, dass das in der Bevölkerung [...] sehr unangenehme Erinnerungen ausgelöst hat." (Int.2, S.11, Z.393-396) Die Serie habe für inn subjektiv keine Erinnerungswerte. Ein Besuch als 17-jähriger im Konzentrationslager Mauthausen habe jedoch im Gegensatz zu der Serie bleibende Erinnerungen hinterlassen, sogar an das Wetter an diesem Tag könne er sich noch erinnern (vgl. Int.2, S.15, Z.527-531).

Für eine andere Mitarbeiterin hat die Serie den Beginn der Auseinandersetzung mit der Thematik ausgelöst (vgl. Int.3, S.12, Z.415-416). "Das war bei mir absolut genau der Auslöser, das hat mich wirklich massiv geprägt. Also das war [...] ein absolut einschneidendes Erlebnis." (Int.3, S.12, Z.420-421) Sie beschreibt die Serie als emotionalen Anstoß, sich mit der Thematik zu befassen, es sei zwar eine plakative und einfache Geschichte gewesen, dennoch wurden die Menschen dadurch vielleicht sogar besser und nachhaltiger als durch wissenschaftliche Aufarbeitung erreicht (vgl. Int.3, S.12-13, Z.424-440). Eine weitere Mitarbeiterin berichtet, dass sie die Serie ebenfalls nicht gesehen hat. Die Dokumentation ,Shoah' hätte sie dagegen sehr 
geprägt und zum ersten Mal mit der Thematik konfrontiert (vgl. Int.6, S.10, Z.353355).

Frau Mag. Lessing erzählt, dass im Jahr 1978 der Opfermythos in Österreich noch sehr verbreitet war. Für ihre Generation sei die Serie der erste Schritt einer Auseinandersetzung mit Einzelschicksalen gewesen. Sie erinnert sich an Diskussionsrunden im Fernsehen nach Ausstrahlung der Serie, in denen die Menschen weinten und erst das Ausmaß des Holocaust begriffen (vgl. Lessing, S.89, Z.285-305). „[...] für mich und meine Klasse, meine Generation, war das wirklich [...] nachvollziehbar an zwei Familien, in die man sich [...] hineinleben konnte." (Lessing, S.8, Z.292-293) Heute sei man sich in der Vermittlung und in der Gestaltung von Ausstellungen $\mathrm{zu}$ der Thematik bewusst, dass die Auseinandersetzung mit Einzelschicksalen ein Ansatz ist, mit dem man die Menschen konkreter erreichen kann als allein durch die Zahl von 6 Millionen Toten (vgl. Lessing, S.8-9, Z.293-295).

Natürlich ist ein Foto von einem Leichenhaufen bei der Befreiung von Auschwitz, wo ein Bagger [...] diese leblosen dünn, dürren Körper einfach hochhebt, [..] grauenhaft, aber 'Holocaust' hat uns die Möglichkeit gegeben, die Familie Levy und die Familie Weiß, mit innen mit zu leiden und zu verstehen, das sind ganz normale Familien gewesen, mit all ihren Verstrickungen mit, [...] Nicht-Juden verheiratet, Nicht-Jüdinnen in dem Fall, [...] dieser Zugang heute über die Einzelschicksale, das hat, glaube ich, wirklich das sozusagen ausgelöst [...] (Lessing, S.9, Z.296-302)

Der Perspektivenwechsel, den 'Holocaust' vor allem auf der persönlichen Ebene der Gesellschaft ausgelöst hat, war auch für Frau Mag. Lessing spürbar.

[...] das war, glaube ich, wirklich der Anfang dessen, dass man sich bewusstgemacht hat, dass das nicht irgendjemand war, [...] was ,Holocaust‘ verursacht hat, ist eben, dass nicht irgendjemand deportiert worden ist und 
nicht von irgendjemanden verpfiffen worden ist, sondern dass es der Nachbar war, der dann einfach auch gleich in deine Wohnung eingezogen ist. Der hat zugeschaut, während du auf den Lastwagen gesetzt worden bist und in das KZ zur Ermordung geschickt worden bist und hat sich in dein Ehebett hineingelegt, das war, [...] für viele Menschen dann plötzlich auch so, die Wohnung [...], in der meine Oma wohnt, ist das vielleicht so eine Wohnung? Also es hat so wahnsinnig viele ganz persönliche Geschichten ausgelöst. Das war für mich ,Holocaust'. (Lessing, S.9, Z.314-323)

Frau Dr. Meissner hat die Serie nicht gesehen, ist aber der Meinung, dass die Serie zeitgeschichtlich sehr wertvoll ist (vgl. Meissner, S.20, Z.729-732). Es sei für sie ein Zeitzeugnis, das für die Wissensvermittlung wichtig sei (vgl. Meissner, S.21, Z.744750).

\section{Die Gründung des Nationalfonds}

Der Gründungsmoment des Nationalfonds kann nach Frau Dr. Uhl im damaligen gesamteuropäischen Prozess in Zusammenhang mit dem damaligen EU- Beitritt Österreichs und der Rede von Dr. Vranitzky als Meilenstein gesehen werden (vgl. Uhl, S.4, Z.116-125).

Obwohl nach den Geschehnissen um Waldheim und Vranitzky bereits etliche Vertriebene wieder langsam begonnen hatten, Österreich zu besuchen, so entstand ein reger Besucherandrang erst durch die Gründung des Nationalfonds. Frau Mag. Lessing erinnert sich: "[...] ich erinnere mich an die ersten Tage im Nationalfonds und die ersten Monate, [...] wir haben Nummern ausgegeben, weil so viele Leute gekommen sind, die wollten reden." (Lessing, S.2, Z.39-41)

Für Frau Dr. Meissner konnte die Entstehung des Nationalfonds nur durch die vorangegangenen Ereignisse erfolgen. Es entstand, auch durch die vielbeachtete Rede des damaligen Bundeskanzlers Dr. Franz Vranitzky, langsam das Bewusstsein, dass ÖsterreicherInnen nicht nur Opfer, sondern auch Täter waren. Erst durch die 
Arbeit des Nationalfonds und seiner Leistung in der Anerkennung von verschiedenen Opfergruppen konnte auch hier das Bewusstsein geschärft werden. "[...] es gab ja auch nicht bedachte Opfergruppen im Gesetz bzw. es gab auch Menschen, die verfolgt wurden und die sich selbst nicht als Opfer gesehen [haben], zum Beispiel die Kinder vom Spiegelgrund." (Meissner, S.21, Z.764-766) Im Sinne der Opferanerkennung habe der Nationalfonds einen ganz wesentlichen Beitrag für die Stärkung und auch Erweiterung der Erinnerungskultur geleistet (vgl. Meissner, S.22, Z.777-781).

\subsubsection{Kategorie 7: Erinnerungskultur - Wandel und Funktion}

Die detaillierten Aussagen zu Wandel und Funktion der Erinnerungskultur ergaben die Unterteilung der zwei Themenbereiche in zwei Subkategorien. In diesem Zusammenhang wurde auch nach den Leistungen des Nationalfonds für die österreichische Erinnerungskultur gefragt und welche Bedeutung Erinnerungskultur persönlich für die Befragten hat. Auch hier ergaben sich zwei Subkategorien.

\section{Wandel und Veränderung der Erinnerungskultur}

Der Wandel der Erinnerungskultur zeigt sich nach Frau Dr. Janistyn-Novák unter anderem auch in der Veränderung der Begrifflichkeiten. Wurde früher von Entschädigung der Opfer gesprochen, so hat der Nationalfonds von Gesten und Anerkennung gesprochen, dies hätte eine Signalwirkung gehabt (vgl. JanistynNovák, S.6, Z.214-217).

Die Erinnerungskultur habe sich vor allem in den letzten 20 Jahren deutlich verändert. Diese Entwicklung sei sehr erfreulich und hänge auch mit der Bereitstellung von Mitteln zur finanziellen Förderung von Erinnerungsprojekten zusammen, wie Frau Mag. Maschke betont (vgl. Maschke, S.7, Z.238-241).

Auch Frau Dr. Uhl zufolge hat sich die Erinnerungskultur deutlich verändert, sie sei zum „[...] europäischen Mainstream [...]“ (Uhl, S.9, Z.309) geworden. Es sei jedoch fraglich, welche Auswirkungen politische Veränderungen - sie spricht hier die Erstarkung der europäischen rechtspopulistischen Strömungen an - auf die Zukunft 
der Erinnerungskultur haben werden (vgl. Uhl, S.9, Z.306-314). Eine Erschöpfung der Erinnerungskultur sehe sie in Zukunft nicht (vgl. Uhl, S.11, Z.398-399).

Einer Mitarbeiterin zufolge zeigt sich der Wandel vor allem in der vermehrten Vielfalt und im Umfang der Veranstaltungen und Projekte (vgl. Int.1, S.6, Z.213-215). Es habe seit der Waldheim-Affäre auch durch den Generationenwechsel eine zivilgesellschaftliche Entwicklung stattgefunden, berichtet ein anderer Mitarbeiter (vgl. Int.2, S.8, Z.273-284). Eine weitere Veränderung sei in der Form des Gedenkens zu bemerken. Es gäbe heute vielfältigere, lokale Projekte, die aus Eigeninitiativen der Bevölkerung entstünden (vgl. Int.2, S.8, Z.284-287). Für inn sei ein negativer Wandel der Erinnerungskultur jedoch in der Politik zu erkennen. "[...] die Lippenbekenntnisse der Politik. [...] Da hat sich aber nichts verändert, es ist nur vielleicht in seiner Aufdringlichkeit stärker geworden, wenn man es sich nicht mehr leisten kann, dazu zu schweigen." (Int.2, S.8, Z.287-291) Positiv sei zu bemerken, dass trotz einem scheinbar fehlenden politischen Konsens dennoch Gelder zur Verfügung gestellt werden würden (vgl. Int.2, S.8, Z.291-302).

Eine andere Mitarbeiterin sieht den positiven Wandel der Erinnerungskultur darin, dass nach der Waldheim-Affäre mehr Bewusstsein in der Gesellschaft und in der Öffentlichkeit für die Thematik entstanden ist (vgl. Int.3, S.10, Z.347-353). Der Nationalfonds habe ebenfalls zu diesem Wandel der Erinnerungskultur beigetragen. Durch die Projektförderungen und vor allem auch durch die Anerkennung der Opfergruppen wie z.B. der Kinder vom Spiegelgrund oder der Wehrmachtsdeserteure konnte die Wahrnehmung in der Öffentlichkeit verändert werden (vgl. Int.3, S.10-11, Z.354-369). Erinnern sei jedoch nicht immer ein Allheilmittel. Die Mitarbeiterin merkt unter Bezugnahme auf das Buch von Christian Meier 'Das Gebot zu Vergessen und die Unabweisbarkeit des Erinnerns' an, dass nach dem Zweiten Weltkrieg die Verdrängung der Geschehnisse für eine gewisse Zeit lang notwendig gewesen wäre.

[...] ich glaube aber auch, dass eine Gesellschaft nur ein gewisses Maß an Grauen tragen kann und dass Vergessen ein [...] Tool ist, ein [...] Hilfsmittel, 
um [...] etwas erträglich zu machen und ich glaube, das war auf Seiten der Opfer [...] auch [...] (Int.3, S.18, Z.624-627)

Der Wandel der Erinnerungskultur sei auch mit dem Wandel in der Gesellschaft verbunden.

Ich glaube, dass Gesellschaften auch so etwas Vergleichbares haben, wie eine posttraumatische Belastungsstörung und dass so etwas eben naturgemäß lang nachwirkt und wenn man bedenkt, dass bei Überlebenden [...] diese ganzen Erinnerungen [...] verstärkt wiederkommen, dann glaube ich, dass in Gesellschaften [...] ähnliche Mechanismen wirken und ich glaube, was [...] eine Gesellschaft gut vertragen könnte, wäre eine offene Diskussion, auch [...] über diese Dualität Erinnern - Vergessen und dass beides seinen Platz haben kann. (Int.3, S.18, Z.632-638)

Nach Meinung der Mitarbeiterin müsste man dem Vergessen mehr Raum geben.

[...] man muss sich an der Wirklichkeit orientieren, es nützt nichts, wenn man [..] sozusagen hohe moralische Ansprüche vor sich herträgt und niemanden damit erreicht, ja, oder das Gegenteil erreicht, was noch schlimmer ist. Also die Gefahr sehe ich schon, dass man die Leute überfordert und dass da Gegenreaktionen kommen, die genau das Gegenteil von dem sind, was wir eigentlich erreichen wollen. (Int.3, S.19, Z.662-666)

Es gebe mehr zivilgesellschaftliches Engagement, auch hierdurch zeige sich der Wandel der Erinnerungskultur, erzählt eine andere Mitarbeiterin (vgl. Int.5, S.5, Z.167-172). Aufgrund dieses Engagements von Schulen, Vereinen oder auch Einzelpersonen sei es möglich, Erinnerungskultur abseits von Museen und Gedenkstätten zu vermitteln (vgl. Int.5, S.5, Z.177-182). Eine weitere Mitarbeiterin 
sieht eine Veränderung in der Erinnerungskultur auch hauptsächlich im Anstieg von Privatinitiativen auf lokaler Ebene (vgl. Int.6, S.8, Z.260-267).

Frau Mag. Lessing berichtet, dass der Holocaust in ihrer Schulzeit Ende der 1970er Jahre kein Unterrichtsthema war (vgl. Lessing. S.8, Z.285-286). Auch für sie ist der Wandel der Erinnerungskultur hauptsächlich im gestiegenen Engagement der Zivilgesellschaft zu erkennen, dies würde sich auch in der Projektförderung des Nationalfonds zeigen (vgl. Lessing, S.9-10, Z.328-337).

Laut Meinung von Frau Dr. Meissner zeigte sich der Wandel in der Erinnerungskultur nicht nur durch die Waldheim-Affäre und die Rede des damaligen Kanzlers Vranitzky in Israel, sondern auch durch die Arbeit des Nationalfonds. Frau Dr. Meissner zufolge hat die Anerkennung der verschiedenen Opfergruppen zu einer Veränderung in der Gedenk- und Erinnerungskultur geführt (vgl. Meissner, S.21, Z.757-780).

\section{Funktion von Erinnerungskultur}

Auf die Frage, ob sich die Erinnerungskultur erschöpfen kann, antwortet ein Mitarbeiter, dass dies seiner Meinung nach nicht passieren könne. „Spricht doch ziemlich viel aus der Holocaustforschung, [...] dagegen, weil [...] sich diese Erinnerung auch durch das Nicht-Erinnern und Nicht-Weitergeben der Informationen innerhalb der Familie [...] nicht erschöpft." (Int.2, S.12, Z.410-414)

Dennoch sei die jetzige Bedeutung und Funktion der Erinnerungskultur kein Garant für die Zukunft.

Es ist keine Garantie dafür, dass diese Erinnerung, sagen wir 2038 oder 2042 zum 100. Jahrestages der Wannseekonferenz oder zu Beginn des [...] Zweiten Weltkrieges [...], dass das dann noch diese Bedeutung hat, die wir gerne hätten oder die heutige Bedeutung. (Int.2, S.13, Z.464-467)

Einer anderen Mitarbeiterin zufolge müssen neue Wege in der Vermittlung gefunden werden, um einer Erschöpfung entgegenzuwirken. Erinnerungskultur würde für die 
heutige Jugend weniger greifbar werden, Erinnerungen würden durch den Lauf der Zeit verblassen (vgl. Int.3, S.13, Z.449-457). Die Herstellung eines Gegenwartsbezugs ist in diesem Zusammenhang auch für eine andere Mitarbeiterin von großer Bedeutung (vgl. Int.4, S.5, Z.175-186). Für sie liegt die Funktion der Erinnerungskultur darin, eine Wiederholung der Vergangenheit zu verhindern (vgl. Int.4, S.6, Z.194-197).

Eine weitere Mitarbeiterin sieht die Funktion der Erinnerungskultur im Respekt den Überlebenden gegenüber begründet. Auch ihrer Meinung nach kann sich Erinnerungskultur erschöpfen, wenn es nicht gelingt, einen Bezug zur Gegenwart herzustellen (vgl. Int.5, S.6-7, Z.217-228). Für eine andere Mitarbeiterin steht fest, dass sich die Erinnerungskultur in ihrer Funktion nicht erschöpfen könne, da Relevanz und Kontext nach wie vor gegeben wären (vgl. Int.6, S.10, Z.336-345).

\section{Die Leistungen des Nationalfonds für die Erinnerungskultur}

Der Nationalfonds ist für Frau Dr. Janistyn-Novák "[...] einer der Garanten dafür, dass das Thema des Nationalsozialismus nicht [...] ritualisiert abgehandelt wird." (Janistyn-Novák, S.14, Z.480-481) Er sei eine anerkannte Institution geworden, betont sie (vgl. Janistyn-Novák, S.16, Z.585).

Die Projektförderungen des Nationalfonds, die auch kleine private Initiativen fördern, charakterisieren laut Frau Dr. Uhl eine zentrale Leistung des Nationalfonds für die Erinnerungskultur (vgl. Uhl, S.6, Z.191-195). Für sie ist der Nationalfonds in erster Linie eine Förderinstitution (vgl. Uhl, S.6, Z.206-207).

Das lange Bestehen des Nationalfonds, seine Etablierung, Expertisen und Erfahrungswerte stellen eine beachtliche Leistung für die Erinnerungskultur in Österreich dar, betont eine Mitarbeiterin (vgl. Int.1, S.8, Z.278-281).

Die Gründung des Nationalfonds hat auch die wissenschaftliche Aufarbeitung des Holocaust gefördert. Dr. Janistyn-Novák nennt hier als Beispiel die Historikerkommission (vgl. Janistyn-Novák, S.8, Z.273-277). 
Durch die Sammlung von Lebensgeschichten könne man die "[...] österreichische Geschichte immer wieder lebendig halten." (Lessing, S.15, Z.520-521) Für Frau Mag. Lessing ist dies etwas, "[...] was wir am besten leisten können." (Lessing, S.15, Z.521-522) Der Versand der publizierten Bände der Lebensgeschichten der Opfer ist für einen weiteren Mitarbeiter ebenfalls eine beeindruckende Leistung. Es seien mittlerweile zehntausende Bücher verschickt worden (vgl. Int.2, S.15, Z.550-552). Für inn leistet auch das Findbuch einen maßgeblichen Beitrag zur Gedenkkultur:

Das ist dann Gedenken. Weil irgendjemand könnte einmal die Familienforschung beginnen, in Australien, in Österreich oder [...] in Israel und hat auf einen Schlag plötzlich Personen, die er gar nicht kannte und wenn er sie nicht gekannt hat, [...] hat er plötzlich statt zwei oder drei, zwölf Ansatzpunkte, um weiter zu recherchieren. Ich habe sie damit, wir haben sie damit zurückgeholt. (Int.2, S.18, Z.644-648)

Als Verdienst für die Erinnerungskultur bezeichnet eine andere Mitarbeiterin ebenfalls die Projektförderungen, aber auch die Vermittlungsarbeit und die Verbreitung von Wissen. Diese Leistungen würden in Zukunft noch weiter ausgebaut werden können (vgl. Int.3, S.15-16, Z.541-556). Eine weitere Mitarbeiterin betont ebenfalls die Ausbaufähigkeit der Projektförderungen (vgl. Int.6, S.9, Z.306-307). Sie würde sich wünschen, dass die MitarbeiterInnen des Nationalfonds „[...] als Botschafter agieren und ein bisschen mehr, vor allem in den Schulen aber auch in den Regionen und [...] Bundesländern mehr [...]“ (Int.6, S.9, Z.308-309) Präsenz zeigen könnten.

Die umfangreiche Sammlung von historischem Wissen und vor allem auch persönlichen Dokumenten im Archiv des Nationalfonds bezeichnet eine Mitarbeiterin als Möglichkeit der zukünftigen Wissensvermittlung (vgl. Int.4, S.7-8, Z.258-268). Den gewaltigen Umfang und die Einzigartigkeit des Archivs des Nationalfonds, das wissenschaftlich aufgearbeitet werden könne, charakterisiert auch eine andere Mitarbeiterin als Leistung für die Erinnerungskultur (vgl. Int.5, S.7, Z.253-261). Für sie wäre die Vermittlung des im Nationalfonds zusammengetragenen Wissens ein Beitrag, den der Nationalfonds in Zukunft im Sinne einer verstärkten 
Öffentlichkeitsarbeit für die österreichische Erinnerungskultur leisten könne (vgl. Int.5, S.8, Z.266-272).

Als Leistungen für die Erinnerungskultur nennt auch Frau Mag. Lessing die Projektförderungen, die auch Projekte aus der Zivilgesellschaft unterstützen, die Digitalisierung des Archivs und den Erfolg der Publikationen des Nationalfonds (vgl. Lessing, S.15, Z.522-534).

Für Frau Dr. Meissner besteht ein wesentlicher Beitrag zur Erinnerungskultur in der Anerkennung der verschiedenen Opfergruppen. Im Laufe der Zeit sei somit für die lange nicht anerkannten Opfergruppen wie die Wehrmachtsdeserteure eine erweiterte Erinnerungskultur entstanden, die auch zu vielen Gedenkprojekten geführt habe (vgl. Meissner, S.22, Z.777-793). Die Leistung des Nationalfonds bestehe darin, "[...] was er für die Gesellschaft leisten kann, durch Erinnern, Gedenken, Vermitteln und die Unterstützung [...] von den verschiedensten Gedenk- und Erinnerungs- und wissenschaftlichen Projekten, weil die Wissenschaft ja dann wiederum Arbeit leistet für die Erinnerungs- und Gedenkkultur." (Meissner, S.25, Z.892-895)

Durch die Projektförderungen des Nationalfonds könne die zivile Gedenkkultur maßgeblich unterstützt werden, erklärt Frau Dr. Meissner (vgl. Meissner, S.22, Z.798-807). Auch inhaltlich leiste der Nationalfonds viel für die Erinnerungskultur.

[...] durch die Herausgabe von lebensgeschichtlichen Zeugnissen, durch die Gestaltung von Workshops, die wir auch anbieten, für Lehrerinnen und Lehrern, wie sie lebensgeschichtliche Zeugnisse den Schülerinnen und Schülern vermitteln können, durch die Aufarbeitung seiner Registratur und Archivs und in späterer Folge sicher durch die Zurverfügungstellung von Materialien für die Wissenschaft und vielleicht auch in Form von kleinen eigenen Projekten. (Meissner, S.24, Z.873-878)

Die Tätigkeiten der Mitarbeiterlnnen des Nationalfonds, die ihr Wissen im Rahmen von Vorträgen, Workshops, Präsentationen und Veranstaltungen der Öffentlichkeit 
vermitteln, trügen zum Erhalt der Erinnerungskultur bei (vgl. Meissner, S.24-25, Z.882-895).

\section{Persönlicher Bezug der InterviewpartnerInnen zu Erinnerungskultur}

Im folgenden Kapitel wird die persönliche Bedeutung von Erinnerungskultur für die Befragten durch einige Zitate hervorgehoben.

Je länger man damit arbeitet, desto mehr nimmt es [...] natürlich Platz im Leben ein und $[\ldots]$ ich glaube, für mich bedeutet es etwas, $[\ldots]$ wo ich darauf achten muss, $[\ldots]$ es im richtigen Verhältnis zu sehen. Erinnern und Gedenken ist wichtig, aber [...] man muss sehr darauf achten, dass man andere nicht überfährt damit, [...] wenn du lange mit der Vergangenheit arbeitest, dann ist die Gefahr, dass du zuviel [...] durch die Brille der Vergangenheit siehst und [...] das könnte auch zu einer gewissen [...] Hybris führen [...] (Int.3, S.16, Z.560-569)

"[...] man sollte immer daran erinnert werden, in der Hoffnung, dass es niemals wieder so weit kommt." (Int.1, S.9, Z.301-302)

Verantwortung zu unternehmen. Das ist für mich ganz etwas Wichtiges. [...] und das hat mich wirklich durch die Jahre immer mitgetragen, [...] sagen zu können, ich bin hier, ich beschäftige mich mit dem Thema Holocaust, mit dem Thema Erinnern, Gedenken. Um das [...] Wissen zu vermitteln und aufrecht zu erhalten und weiter zu geben. (Int.4, S.8, Z.272-278)

"Am ehesten Leute - verstorbene, ermordete, verfolgte - in irgendeiner Weise ins Gedächtnis zurückzuholen." (Int.2, S.17, Z.605-606) 
[‥] für mich ist es natürlich immer wieder dieser Weg über meine Großmutter, dass ich sie einfach am Leben erhalte. [...] wenn ich ihren Namen bei einer Veranstaltung nenne, dann ist es für mich was ganz, ganz Persönliches. [...] da habe ich noch immer das Gefühl, dass ich ihr ein bisschen gerecht werde, weil ich einfach sie aus der Vergessenheit heraushole, das ist für mich Erinnerungskultur, dass jeder und jedes Opfer [...] ein Recht darauf hat [...]. Wir müssen uns erinnern, weil hier sind Menschen grausamst ermordet worden [...]. Wenn wir uns nicht mehr darum kümmern, zu sagen, das ist damals passiert, dann werden sie ein zweites Mal ermordet und das ist für mich Erinnerungskultur, das ist eine Verpflichtung [...] (Lessing, S.15-16, Z.542-553)

"Also für mich bedeutet Erinnern [...], dass man Dinge, die sich verändern, zuerst einmal festhalten muss, bevor sie verschwinden. Es bedeutet, Welten festzuhalten für die kommenden Generationen, auch für ihre eigenen Wurzeln, damit sie andocken können." (Meissner, S.26, Z.923-927)

Es bedeutet, Entwicklungen zu sehen und [...] sich zu erinnern, damit Dinge nicht wieder geschehen, also um aus den Dingen zu lernen, aber was die persönliche Erinnerung für die Leute betrifft, ihr Leben und ihr Wirken einfach für die Nachwelt bewusst zu machen, [...], aber auch einfach Schicksale nachvollziehbar zu machen und zu gedenken, [...] sie zu würdigen, also Erinnerungskultur ist für mich auch eine Würdigungskultur [...] (Meissner, S.26, Z.927-938) 


\subsubsection{Kategorie 8: Kritik an Erinnerungskultur}

In dieser Kategorie wird auf die Kritik an der Erinnerungskultur, wie es auch im theoretischen Teil der vorliegenden Arbeit erläutert wurde, eingegangen. Die Meinungen und Bewertungen der befragten Personen zu Instrumentalisierung, Institutionalisierung und Ritualisierung der Erinnerungskultur werden in diesem Kapitel zusammengefasst. In diesem Zusammenhang wird auch der Erfolg der Erinnerungskultur angesprochen.

Eine Ritualisierung der Erinnerungskultur hat nach Frau Dr. Janistyn-Novák den Vorteil der Kontinuität. Wichtig sei nur darauf zu achten, dass es nicht inhaltslos werde, sondern dennoch eine Auseinandersetzung mit der Thematik stattfinde (vgl. Janistyn-Novák, S.10, Z.348-357).

Frau Mag. Maschke sieht auch Vorteile in der hohen medialen Präsenz von Erinnerung, vor allem im Gedenkjahr 2018. Es sei legitim, solch einschneidende Jubiläen zu begehen. Um eine Breitenwirksamkeit zu erlangen, könne man durch Projekte und medienwirksames Auftreten punkten (vgl. Maschke, S.4, Z.130-134). Für sie ist es selbstverständlich, dass junge Menschen durch die zunehmende zeitliche Distanz immer weniger an das Geschehene andocken können. Dennoch präge das Geschehene Gesellschaften über Generationen hinweg. Bildungsverantwortliche müssten sich dessen bewusst sein und daher gelte es Instrumente zu finden, die junge Menschen anregen, anhand der Geschehnisse der Vergangenheit zu reflektieren, wie sie gegenwärtigen rassistischen Erscheinungsformen, wie etwa Antisemitismus und anderen entgegentreten können (vgl. Maschke, S.4-5, Z.143-166).

Die Kritik an der Erinnerungskultur könne auch auf den Erfolg derselben zurückzuführen sein und eine Institutionalisierung und Ritualisierung wäre somit ein Ergebnis dieses Erfolgs, erläutert Frau Dr. Uhl. „Also das würde ich in dem Sinn nicht negativ sehen, sondern die Absenz von Institutionalisierung und Ritualisierung wäre ein Problem [...]." (Uhl, S.9, Z.323-325) 
In ihren Ausführungen verweist Frau Dr. Uhl auf Assmanns Buch 'Das neue Unbehagen an der Erinnerungskultur'. Die Kritik würde oft mit diesem Unbehagen an einer Auseinandersetzung mit der Thematik im Sinne eines Erschöpfungsnarrativs einhergehen. Wenn es jedoch keine erfolgreiche Erinnerungskultur geben würde, wäre auch eine kritische Auseinandersetzung damit nicht möglich (vgl. Uhl, S.12, Z.418-424).

Für eine Mitarbeiterin ist die Kritik an der Überpräsenz der Erinnerungskultur nachvollziehbar. Sie erachtet eine zukünftige Auseinandersetzung damit dennoch als sehr wichtig, um nachfolgenden Generationen die Thematik weiter vermitteln zu können, besonders in der Zukunft, wenn keine ZeitzeugInnen mehr am Leben sein werden (vgl. Int.1, S.7, Z.225-230). Ein anderer Mitarbeiter sieht die Kritik an der Erinnerungskultur vor allem im übermäßigen, akademischen Zugang. Das nahende Ende der Zeitzeuglnnen-Ära würde zu einem abstrakteren Erinnern führen (vgl. Int.2, S.9, Z.320-323). Durch technische Konstrukte, wie die Hologramme von Überlebenden in der USC Shoah Foundation ${ }^{103}$, würde versucht werden, den Verlust der ZeitzeugInnen zu kompensieren. Dies könne jedoch nicht die authentischen Berichte der Überlebenden ersetzen (vgl. Int.2, S.9-10, Z.327-338). Auch für inn bedeutet Ritualisierung nicht automatisch Kritik. Man müsse zwischen Ritus, der eine gesellschaftliche Funktion habe, und Ritualisierung unterscheiden. "[...] es ist ein Ritus, der gesellschaftlich etwas bringt, dann ist das etwas anderes, als zum Ritual Erstarrtes, dem wir uns, weil es der Tag ist, aussetzen." (Int.2, S.10, Z.349-351) Kritik an einer Institutionalisierung sei ebenfalls vorsichtig $z u$ bewerten. Gedenkveranstaltungen seien nicht automatisch negativ zu beurteilen, wenn sie von einer Institution initiiert werden würden, auch hier sei vor allem der Inhalt der Veranstaltungen wichtig (vgl. Int.2, S.10, Z.351-361).

Außerhalb ihrer Tätigkeit im Nationalfonds nehme sie schon Kritik wahr, berichtet eine weitere Mitarbeiterin. Andererseits herrsche mehr Verständnis, wenn Menschen konkret durch lokale Projekte zum Beispiel erreicht werden. Die Herausforderung sei, Menschen direkt zu erreichen (vgl. Int.3, S.11, Z.377-384). Sie erläutert, dass durch

\footnotetext{
${ }^{103}$ Anm. d. Verf.: Die USC Shoah Foundation - the Institute for Visual History and Education ist eine, von Stephen Spielberg im Jahr 1994 gegründete gemeinnützige Organisation, die mittlerweile an der University of Southern California angesiedelt ist. Die Organisation zeichnet Video-Interviews von Überlebenden des Holocaust auf. Siehe auch: https://sfi.usc.edu/about [Datum des Zugriffs: 30.8.2019]
} 
den lokalen Bezug eines Erinnerungsprojektes ein Bewusstsein geschaffen und Geschichte aufgearbeitet werden könne, auch wenn dies manchmal Unbehagen hervorrufen würde. Dieser lokale Zugang sei ihrer Meinung nach eine Herausforderung für die zukünftige Erinnerungskultur (vgl. Int.3, S.11, Z.384-401).

Kritik an der Erinnerungskultur sei berechtigt, wenn Gedenken zum leeren, inhaltslosen Ritual werden würde. Nach Ansicht einer weiteren Mitarbeiterin sollten vor allem Jugendliche über Inhalte und Gefühle erreicht werden, um der immer wiederkehrenden Frage, was das denn noch mit innen $z u$ tun habe, entgegenzutreten (vgl. Int.4, S.5, Z.167-175). Als berechtigt empfindet eine andere Mitarbeiterin die Kritik auf institutionalisierter politischer Ebene. Die Authentizität solcher Gedenkfeiern würde nicht immer gegeben sein. Sie selbst nimmt kleinere Veranstaltungen als authentischer wahr, gerade im Gedenkjahr 2018 habe es mehr Veranstaltungen als sonst üblich gegeben (vgl. Int.5, S.6, Z.190-202).

Eine Mitarbeiterin merkt an, dass Gedenkfeiern oft aufgesetzt und nicht authentisch wirken würden. Sie ist der Meinung, dass es am besten sei, wenn Gedenkfeiern durch Eigeninitiativen heraus entstünden und den Menschen die Möglichkeit geboten würde, sich selbst einzubringen und Überlegungen anzustellen (vgl. Int.6, S.9, Z.320327).

Frau Mag. Lessing kann die Kritik an einer Überpräsenz in den Medien ebenfalls gut nachvollziehen. Besonders im Gedenkjahr 2018 habe es sehr viele Veranstaltungen gegeben. Eine Institutionalisierung an sich würde sie jedoch nicht kritisieren (vgl. Lessing, S.12, Z.407-415).

Es haben sich Sachen institutionalisiert, aber eine Institutionalisierung von Gedenken ist nicht automatisch etwas Schlechtes, es kommt darauf an, [...] zum Beispiel das Fest der Freude ist ein tolles Gedenken, weil es ist wirklich die klare Darstellung, dass der 8. Mai nicht ein Besatzungstag war, sondern ein Befreiungstag und ein Fest der Freude. (Lessing, S.12, Z.415-419) 
Solange noch ZeitzeugInnen am Leben seien und bei Veranstaltungen sprechen könnten, könne man institutionalisierte Gedenkfeiern sinnvoll gestalten. Auch Mag. Lessing zufolge müsse man jedoch darauf achten, dass Gedenken nicht zu einer leeren Hülle werde (vgl. Lessing, S.12, Z.420-426). Die Fülle an Gedenkveranstaltungen könne durchwegs Überforderung beim Einzelnen erzeugen, erläutert Frau Dr. Meissner. Sie ist jedoch der Meinung, dass Rituale gebraucht werden. Es könne als Motivation betrachtet werden, eigene Projekte ins Leben zu rufen (vgl. Meissner, S.23, Z.817-832).

\subsubsection{Kategorie 9: Herausforderungen und Zukunftsperspektiven für die Erinnerungskultur}

Die Herausforderungen und Zukunftsperspektiven für die österreichische Erinnerungskultur bilden die letzte Kategorie der Datenauswertung. Für diese Kategorie ergaben sich anhand der differenzierten Aussagen vier Subkategorien. Eine Subkategorie fasst die Aussagen zu möglichen Gefahren für den Erhalt der Erinnerungskultur zusammen. Eine weitere Subkategorie, welche induktiv aufgrund der Aussagen einiger befragter Personen entstanden ist, befasst sich mit möglichen zukünftigen Wirkungsstätten der Erinnerungskultur. Die letzten beiden Subkategorien enthalten Antworten auf die Fragen, welche zukünftigen Aufgaben die Erinnerungskultur haben wird und welche dieser Aufgaben vom Nationalfonds erfüllt werden könnten.

\section{Gefahren für den Erhalt der Erinnerungskultur}

Eine Gefahr für den Erhalt der Erinnerungskultur liegt Frau Mag. Maschke zufolge in der immer größer werdenden zeitlichen Distanz der Geschehnisse und der dadurch entstehenden Frage der „Holocaust-Distortion“, einer möglichen Leugnung und Verzerrung des Holocaust, die auch international diskutiert wird (vgl. Maschke, S.7, Z.245-253).

Für Frau Dr. Uhl könnte der Erfolg der Erinnerungskultur auch eine Gefahr bergen. Wenn das Gedenken an die Opfer des Holocaust von einer Gegenkultur zu einem „[...] Teil der staatlichen Repräsentationskultur wurde, ist es natürlich [...] ein auch staatliches Projekt." (Uhl, S.12, Z.431-432) Ein daraus entstehendes Problem wäre 
folglich die Möglichkeit der Erstarrung eines offenen Diskurses in Pathosformeln (vgl. Uhl, S.12, Z.432-433).

Man müsse darauf achten, dass die Erinnerungskultur nicht zu einem leeren Gedenken wird, hält Frau Mag. Lessing auch in diesem Zusammenhang fest (vgl. Lessing, S.10, Z.343-344). Um die Gefahr eines hüllenlosen Gedenkens zu vermeiden, dürfe man nicht aufhören, die Zugänge zur Erinnerungskultur weiterzuentwickeln (vgl. Lessing, S.11, Z.376-382). Die größte Gefahr für den Erhalt der Erinnerungskultur stellt für Frau Dr. Meissner das Ende der ZeitzeugInnen-Ära und das damit einhergehende schwindende Interesse an der Thematik dar. Es müssten neue Zugänge gefunden und entworfen werden (vgl. Meissner, S.25, Z.900910).

\section{Zukünftige Wirkungsstätten für Erinnerungskultur}

Das fehlende Holocaust-Museum stelle eine Lücke in der österreichischen Erinnerungslandschaft dar, hält Frau Dr. Uhl fest (vgl. Uhl, S.7, Z.238). In Österreich beziehungsweise in Wien gebe es keinen zentralen Ort wie zum Beispiel das NSDokumentationszentrum in München. Laut Frau Dr. Uhl ist die Situation dadurch gekennzeichnet, dass verschiedene Museen und Institutionen die Aufgabe übernehmen, sich mit der Geschichte des Holocaust auseinandersetzen, etwa das Wien Museum, das Jüdische Museum und das Haus der Geschichte Österreich am Heldenplatz in seinen Wechselausstellungen (vgl. Uhl, S.7-8, Z.248-264).

Auch ein Mitarbeiter kann sich eine zukünftige, zentrale Forschungs- und Wirkungsstätte, angelehnt an das Haus der Geschichte, für die Erinnerungskultur vorstellen (vgl. Int.2, S.14-15, Z.518-524).

\section{Zukünftige Aufgaben der Erinnerungskultur}

Für Frau Dr. Janistyn-Novák ist der Erhalt der Erinnerungskultur eine Verpflichtung, um den Opfern weiterhin zu gedenken und eine Warnung, die Opfer nicht zu vergessen (vgl. Janistyn-Novák, S.12, Z.436-441). Für Frau Mag. Lessing drückt die Erinnerungskultur den Überlebenden Respekt aus. „Ich finde noch immer, solange wir Überlebende haben, muss man innen nicht nur aus Respekt den Raum geben, 
dass sie eine Nachricht an die Jugend richten können, sondern auch, weil sie auf einer ganz anderen emotionalen Basis Menschen erreichen." (Lessing, S.12-13, Z.438-441)

Für die zukünftigen Aufgaben der Erinnerungskultur brauche es eine disziplinenübergreifende Zusammenarbeit von ExpertInnen (vgl. Janistyn-Novák, S.15, Z.523-530). Für sie liegt ein zentraler Ansatz in der Auseinandersetzung mit Jugendlichen, auch durch die Möglichkeiten der Kanäle der sozialen Netzwerke (vgl. Janistyn-Novák, S.16, Z.578-583).

Die Vermittlung von demokratischen Haltungen sowie die Herstellung eines Gegenwartsbezugs zu aktuellen Fluchterfahrungen und damit zusammenhängenden Traumata stellt für Frau Mag. Maschke einen Auftrag an die Erinnerungskultur in Zukunft dar (vgl. Maschke, S.5, Z.153-163). Die vielfältigen Erinnerungen einer heterogenen Gesellschaft müssten miteinander verknüpft werden. Gegenwartsgeschichten von Menschen, die sich auf der Flucht befinden und auch zu uns kommen, könnten zur Unterstützung von historischem Lernen herangezogen werden (vgl. Maschke, S.8-9, Z.291-297). Biographisches Lernen sei ihr zufolge eine gute Möglichkeit für die Vermittlungsarbeit. Schülerinnen und Schüler könnten sich mit Einzelschicksalen auseinandersetzen und mit eigenen Erfahrungen in Bezug setzen und so aus der Vergangenheit lernen (vgl. Maschke, S.5-6, Z.182-188). Für Frau Mag. Maschke ist Erinnern durch die ZeitzeugInnenschaft stark geprägt. Geschichten über Flucht und Ausgrenzung würden wichtige Möglichkeiten bieten, sich mit der Vergangenheit auseinanderzusetzen. Leider müsse man sich überlegen, wie diese Auseinandersetzung und das Lernen aus der Vergangenheit auch in Zukunft weiter gewährleistet werden könne. Sie nennt hier Möglichkeiten durch didaktisch aufbereitete und mittels moderner Technologien konservierte Interviews, wie sie auch von _erinnern.at_zur Verfügung gestellt werden (vgl. Maschke, S.6, Z.198-205).

Es gibt auch laut Frau Mag. Lessing bereits einige Überlegungen für die Zukunft ohne ZeitzeugInnen. Als Beispiel nennt sie ebenfalls die Hologramme der USC Shoah Foundation. Einem Überlebenden werden ca. 1500 Fragen gestellt, er wird in einem 360 Grad Winkel gefilmt und als Hologramm beantwortet der Überlebende 
dann Fragen und spricht zu einem. Mag. Lessing empfindet dies zwar als tolle technische Möglichkeit, vor allem die Jugend könne damit erreicht werden (vgl. Lessing, S.13, Z.447-458). Dennoch steht sie diesem Ansatz auch skeptisch gegenüber.

Ich finde es spooky und ich finde es vielleicht sozusagen gefährlich, weil ich mir denke, was werden Revisionisten sagen, die dann sagen, die haben ja nie existiert, das sind lauter Hologramme, ja? Dann gibt es eben auch Virtual Reality, dann kriegst du [..] eine Brille aufgesetzt und du gehst mit Pinchas Gutter durch Majdanek. Du stehst in der Früh mit inm in seinem Hotelzimmer, [...], dann fährt er mit dir Taxi und fährt ins Konzentrationslager Majdanek und geht mit dir [...] zur Gaskammer und zeigt, wo seine Schwester verbrannt worden ist, [...] das ist so nahe, das ist unglaublich. Wollen wir so einen Erlebnispark? (Lessing, S.13, Z.458-466)

Die Erinnerungskultur habe sich jetzt schon durch Social Media weiterentwickelt. Frau Mag. Lessing nennt hier als Beispiel die QR-Codes, mit denen mobil digitale Informationen zu den Stolpersteinen ${ }^{104}$ aufgerufen werden können (vgl. Lessing, S.10-11, Z.365-373).

Also es gibt so tolle Sachen heute. Das entwickelt sich alles weiter, man darf nicht aufhören, weil sonst irgendwann einmal wird es nur mehr ein Teil eines Geschichtsbuches sein, aber die Erinnerungskultur entwickelt sich gut, weil [..] es sind wie gesagt nicht nur hüllenlose Gedenken, wo man irgendetwas abfeiert, es [...] wird wieder politischer, das Erinnern und das ist auch gut so und [...] ich sehe genug Jugendliche, [...] die bereit sind, sich mit dieser Erinnerungskultur auseinanderzusetzen. (Lessing, S.11, Z.376-382)

\footnotetext{
${ }^{104}$ Anm. d. Verf.: die Stolpersteine sind ein Erinnerungsprojekt, bei dem Pflastersteine, auf denen die Namen und Daten von Opfern des Holocaust eingraviert sind, vor den ehemaligen Wohnhäusern der Opfer in den Boden verlegt werden. https://steinedererinnerung.net/ [Datum des Zugriffs: 30.8.2019]
} 
Wie Erinnerungskultur in Zukunft Jugendlichen generell vermittelt werden könnte, wenn keine ZeitzeugInnen mehr am Leben sein werden, ist dennoch auch für Frau Mag. Lessing eine schwierige Frage. Erinnerungskultur würde sich verändern. Für sie stellen zukünftig vor allem Mahnmale und Erinnerungsorte einen wichtigen Zugang dar, um die Menschen weiterhin für die Thematik zu sensibilisieren (vgl. Lessing, S.10, Z.346-364). Auch laut Frau Dr. Uhl ist die Sorge, weiterhin Erinnerungen und Erinnerungsorte lebendig zu halten, ein zentraler Aspekt für viele Institutionen (vgl. Uhl, S.10, Z.345-351).

Mag. Lessing befürchtet trotzdem, dass der Holocaust irgendwann einmal nur mehr Teil der Geschichtsbücher sein wird, mit zwei institutionalisierten Gedenktagen, dem 27. Jänner anlässlich der Befreiung von Auschwitz und dem 5. Mai anlässlich der Befreiung Mauthausens (vgl. Lessing, S.14, Z.483-489). Wie das Gedenken in 10, 20 oder 30 Jahren aussehen werde und ob Mahnmale oder Stolpersteine irgendwann verschwinden werden würden, bleibe offen (vgl. Lessing, S.14, Z.493-497).

Die zukünftige Gestaltung von Erinnerungskultur sieht eine Mitarbeiterin weiterhin in Veranstaltungen, Schulprojekten, Gedenktafeln, womit die Aufmerksamkeit der Gesellschaft erreicht werden kann (vgl. Int.1, S.8, Z.272-273). Ein weiterer Mitarbeiter betont die Wichtigkeit von Besuchen von Gedenkstätten auch in der Zukunft (vgl. Int.2, S.15, Z.527-531). Für zukünftige Generationen müsse eine Erinnerungskultur weiterhin existieren (vgl. Int.2, S.12, Z.430).

Eine Transformierung der Weitergabe des Wissens ist für eine andere Mitarbeiterin in Zukunft wichtig. Man dürfe die Jugend nicht mit moralisierendem, zwanghaftem Gedenken überfordern. Eine empathische Herangehensweise sei notwendig (vgl. Int.3, S.14, Z.481-491). Sie würde auch einen frühen Beginn der Wissensvermittlung bei Kindern befürworten. "Also da ist noch [...] mehr zu erreichen, wenn man früher ansetzt, dass die [Kinder] ein kritisches Denken entwickeln." (Int.3.S.14, Z.504-505) In Zukunft müsse man auch dem Vergessen und der Verarbeitung Raum geben können, man könne die Menschen nicht zum Erinnern zwingen. Dies würde Abwehrhaltungen und Gegenreaktionen hervorrufen. In Zukunft müsse sich die Erinnerungskultur auch an der Wirklichkeit orientieren (vgl. Int.3, S.18-19, Z.652671). Der Begriff der Erinnerungskultur sei an sich zu vergangenheitsorientiert, 
erläutert sie weiters (vgl. Int.3, S.16, Z.562-564). Es würde durchaus die Möglichkeit bestehen, dass der Begriff ,Erinnerungskultur' eine abschreckende Wirkung habe, die auch als Zumutung empfunden werde, dem gelte es entgegenzuwirken. Für sie sei es letztendlich eine Frage des Marketings (vgl. Int.3, S.16-17, Z.576-587).

Eine zukünftige Erinnerungskultur ohne ZeitzeugInnen wird anders sein, erzählt eine weitere Mitarbeiterin. Erinnern sei wichtig, es gebe Möglichkeiten der Vermittlung auch ohne ZeitzeugInnen. Ihrer Meinung nach ist es essentiell, wachsam gegenüber Tendenzen, die Ausgrenzung zur Folge haben, zu bleiben und einen Gegenwartsbezug der Erinnerungskultur herzustellen (vgl. Int.5, S.7, Z.233-244). Auch Frau Mag. Lessing betont die Wichtigkeit eines Gegenwartsbezugs. Man müsse Parallelen zum heutigen Geschehen ziehen (vgl. Lessing, S.11, Z.382-393).

Eine weitere Mitarbeiterin sieht die zukünftige Erinnerungskultur vor allem durch Schulprojekte weiterhin verankert (vgl. Int.6, S.8, Z.272-276). "Sie erforschen so die Geschichte, werden selber zu Forscher[n] und dann konfrontieren sie sich mit der Vergangenheit der eigenen Familie und das ist etwas ganz [...] Spannendes, was bis [...] vor kurzem vielleicht überhaupt [...] kein Thema war." (Int.6, S.8, Z.286-289)

Nach Meinung von Frau Dr. Meissner wird eine zukünftige Erinnerungskultur ohne ZeitzeugInnen jedenfalls eine Veränderung erfahren. Sie denkt jedoch, dass der persönliche Bezug zu den Einzelschicksalen der Überlebenden und der Opfer aufrechterhalten werden kann. Durch Videointerviews, Audiofiles, durch persönliche Lebensgeschichten und Fotos, wie sie auch der Nationalfonds publiziert, könne die Erinnerung weitervermittelt werden (vgl. Meissner, S.23, Z.837-847). "Es wird eine Veränderung, ich glaube aber trotzdem und es arbeiten wirklich viele daran, dass es weiterhin eine veränderte Erinnerungskultur geben wird [...]" (Meissner, S.24, Z.863864) 


\section{Conclusio und kritische Reflexion}

Die Forschungsergebnisse werden nun zusammengefasst und mit den theoretischen Ausarbeitungen der vorliegenden Arbeit in Zusammenhang gebracht, um anschließend zu einer Beantwortung der Forschungsfrage, was der Nationalfonds aufgrund der gesetzlichen Erweiterung der Aufgabenbereiche für die österreichische Erinnerungs- und Gedenkkultur leisten kann und welche organisatorischen Herausforderungen sich dadurch ergeben, zu gelangen. Um zu einem umfassenden Bild der Leistungen des Nationalfonds zu gelangen, wurden nicht nur die Aufgabenbereiche nach der gesetzlichen Erweiterung von 2017, sondern auch die Veränderungen der Aufgabenbereiche seit der Gründung des Fonds betrachtet. So konnte ein ausführlicher Überblick über die organisatorischen Entwicklungen und Herausforderungen des Fonds gewonnen werden. Die Verankerung des Nationalfonds in der österreichischen Erinnerungs- und Gedenkkultur wurde theoretisch und empirisch aufgearbeitet. Hierzu wurde auch auf die historische Entwicklung der Erinnerungskultur unter Einbeziehung von Erläuterungen zu Traumata und Retraumatisierungen bei Opfern des Holocaust sowie zu verschiedenen Gedächtnistheorien Bezug genommen.

In dieser Arbeit wurden die Entwicklung der österreichischen Erinnerungskultur und die damit zusammenhängenden Schlüsselereignisse erläutert. Es zeigten sich auch etliche Verknüpfungen zwischen Meilensteinen der Erinnerungskultur, die in der Arbeit beschrieben wurden, und dem Nationalfonds. So hat z.B. Generalsekretärin Mag. Hannah Lessing in der Serie „Holocaust - die Geschichte der Familie Weiß“, die zu einem erstmaligen Perspektivenwechsel auf eine opferorientierte Erinnerungskultur führte, mitgewirkt. Die sogenannte Waldheim-Affäre von 1986 wurde ebenfalls als Meilenstein gesehen. Durch die damaligen Geschehnisse kamen Forderungen nach umfassenden Restitutionen wieder zur Sprache und führten in weiterer Folge als Antwort auf die bisher unzureichenden Restitutionsmaßnahmen der Republik zur Gründung des Nationalfonds im Jahr 1995. Als weitere Meilensteine gelten die Stockholm Deklaration und die Gründung der „Task Force for International Cooperation on Holocaust Education, Remembrance, and Research (ITF), welche 2012 in International Holocaust Remembrance Alliance (IHRA) umbenannt wurde. Die österreichische Mitgliedschaft in der IHRA seit 2001 unter der Co-Leitung der 
Generalsekretärin des Nationalfonds, Mag. Hannah Lessing, verdeutlicht die Verbindung zum Nationalfonds ebenfalls.

Der Begriff Erinnerungskultur fand ab den 1990er Jahren Eingang in den Sprachgebrauch und kennzeichnet den Umgang mit der Vergangenheit in Bezug auf Erinnern und Gedenken an den Holocaust. Die Entwicklung einer Erinnerungskultur im Sinne einer Verankerung im kollektiven Gedächtnis war stark mit der offiziellen Haltung Österreichs und auch mit dem Umgang der Gesellschaft mit der eigenen Vergangenheit verknüpft. Die Haltung Österreichs, sich als erstes Opfer des Nationalsozialismus zu sehen und die kaum vorhandene Verantwortungsübernahme verzögerten eine fundierte Auseinandersetzung mit der eigenen Geschichte für lange Zeit. Die Vergangenheit wurde verdrängt, um in die Zukunft blicken zu können, sowohl auf Täter- als auch auf Opferseite. Wie hier beschrieben, war Schweigen eine Form der Vergangenheitsbewältigung für die Gesellschaft einerseits und für die Überlebenden andererseits, die ihre traumatischen Erfahrungen vergessen wollten und sich oft schuldig fühlten überlebt zu haben. Im Hinblick auf die Situation in Österreich in der Nachkriegszeit trugen für viele Überlebende nicht nur die ablehnende Haltung des offiziellen Österreich, sondern auch die fehlende beziehungsweise späte Anerkennung als Überlebende und die mangelnde Behandlung der traumatischen Folgen, die sie aufgrund ihrer Verfolgung erlitten hatten, zu einer vielfachen Manifestierung der Traumatisierungen bei. Das Schweigen der Überlebenden war auch Ausdruck eines Vermeidungsverhaltens, das eines der Symptome der posttraumatischen Belastungsstörung darstellt. Die doppelte Mauer des Schweigens, wie Dan Bar On es bezeichnet hat, konnte durch den Generationenwechsel in den 1960er, 1970er Jahren durchbrochen werden.

Erst durch die beginnende wissenschaftliche Aufarbeitung, die Klassifizierung des Holocaust - Syndroms als Form der Posttraumatischen Belastungsstörung und die Erkenntnis der transgenerationalen Weitergabe von Traumata wurden die weitreichenden Folgen der traumatischen Erlebnisse sichtbar. Die Folgen dieser traumatischen Erlebnisse sind bis heute spürbar. Oftmals kommt es bei Personen mit posttraumatischer Belastungsstörung aufgrund von wegfallenden Bewältigungsstrategien zu einer Retraumatisierung im Alter und/oder zu einer transgenerationalen Weitergabe von Traumata an die Nachkommen. 
Der erste Generationenwechsel ermöglichte den Paradigmenwechsel zu einer opferorientierten Erinnerung. Zu diesem Paradigmenwechsel trug die Ausstrahlung der Serie "Holocaust - die Geschichte der Familie Weiß" im Jahr 1979 bei. Der erstmalige Blick auf die Zeit des Nationalsozialismus aus der Perspektive der Opfer wenn auch nur in einer fiktiven Fernsehserie - hatte große Auswirkungen auf die zivilgesellschaftliche Auseinandersetzung mit der Vergangenheit. Die Ausstrahlung der Serie ist somit zu Recht als Meilenstein in der Entwicklung der Erinnerungskultur anzusehen. Dieser Tatsache wurde auch in den Ergebnissen der Befragungen Rechnung getragen.

Für den Wandel von einer Vergangenheitsbewältigung zu einer Erinnerungskultur war der Perspektivenwechsel auf eine Opferorientierung maßgeblich. Die Gründung des Nationalfonds fiel in die Zeit, in der Österreich die These, das erste Opfer des Nationalsozialismus gewesen zu sein, nicht mehr aufrechterhalten konnte. Ereignisse wie die Waldheim-Affäre, aber auch das offizielle Bekenntnis des damaligen Bundeskanzlers Vranitzky vor dem Parlament im Jahr 1991 bedingten eine beginnende gesellschaftliche Auseinandersetzung mit der Thematik. Die Gründung des Fonds war durch einige Besonderheiten gekennzeichnet und mit etlichen Herausforderungen verbunden. Eine Besonderheit ist, dass der Nationalfonds beim Nationalrat angesiedelt ist und im Verfassungsrang steht. Durch die erstmalige Anerkennung sämtlicher Opfergruppen im Nationalfondsgesetz hat der Nationalfonds essentiell zu einem Paradigmenwechsel beigetragen. Zu den Herausforderungen der ersten Stunde zählte die Tatsache, dass man bei der Beschlussfassung des Nationalfondsgesetzes das Ausmaß der zu bewältigenden Arbeit nicht kannte. Durch die späte Gründung des Fonds entstand ein Zeitdruck, möglichst schnell Entschädigungszahlungen an die noch lebenden Opfer des Nationalsozialismus leisten zu können. Durch diesen Zeitdruck und auch durch die Verantwortung den Überlebenden gegenüber standen die Arbeit und die Auszahlungen immer im Vordergrund. Eine Organisationsstruktur wuchs erst langsam mit der Zeit. Im Laufe seines 24jährigen Bestehens unterlag der Nationalfonds vielen Veränderungen. Die Organisation wuchs aufgrund neuer Aufgaben, die hinzukamen und durch die Gründung des Allgemeinen Entschädigungsfonds und des Fonds zur Instandsetzung der jüdischen Friedhöfe. 
In der Auswertung der Interviewaussagen hat sich ergeben, dass es laut den Befragten in der Anfangszeit des Nationalfonds kaum eine Organisationsstruktur gab. Diese Aussagen müssen allerdings differenziert betrachtet werden. Die Vorgaben durch das Nationalfondsgesetz stellten in Grundzügen eine Organisationsstruktur auf formaler Ebene dar, aber es gab keine weiterführenden Vorgaben, wie die Organisation aufzubauen sei und wie die Abteilungen und Arbeitsabläufe gestaltet werden sollten. Eine interne Organisationstruktur hat sich erst im Laufe der Jahre auch durch Initiativen der MitarbeiterInnen entwickelt, da durch die immer vielfältigeren Aufgabengebiete des Nationalfonds und auch durch die spätere Gründung des Allgemeinen Entschädigungsfonds und des Fonds zur Instandsetzung der jüdischen Friedhöfe in Österreich gewisse Arbeitsstrukturen vonnöten waren.

Die Mitarbeiterlnnen und auch die Geschäftsleitung haben in den Befragungen die anfänglich fehlenden Strukturen als unzureichend bezeichnet, dennoch hat sich gezeigt, dass genau dies zum Erfolg des Nationalfonds geführt hat. Durch das Engagement, die Freiheit in der Gestaltung und die Eigeninitiativen der Belegschaft, sowie dem Wunsch der Geschäftsleitung, keine strengen Hierarchien einführen zu wollen, konnten die zahlreichen Aufgaben mit der nötigen Flexibilität und Professionalität gemeistert werden. Der Nationalfonds hat sich von einer kleinen Organisation, die zu Beginn temporär geplant war und ursprünglich ausschließlich für Entschädigungszahlungen an noch lebende Opfer des Nationalsozialismus sowie für Projektförderungen gegründet wurde, zu einer international anerkannten Institution entwickelt, die in vielen Bereichen einen wichtigen Beitrag zur österreichischen Erinnerungs- und Gedenkkultur leistet.

Zusammenfassend hat der Nationalfonds maßgeblich zum Wandel der Erinnerungskultur beigetragen. Durch die vielfältige Arbeit des Nationalfonds, die beständige Unterstützung durch die Politik und die Etablierung als anerkannte Institution wurde ein stärkeres Bewusstsein für die Thematik geschaffen und die Wahrnehmung in der Öffentlichkeit verändert. Die Definition des Opferbegriffs im Nationalfondsgesetz hat zu einer Erweiterung der Erinnerungskultur geführt. Durch den Nationalfonds haben erstmals Opfergruppen, die vorher nicht als Opfer angesehen wurden, Anerkennung erhalten. Der Nationalfonds zeichnet sich auch durch seine herausragende internationale Vernetzung aus. Die Befragungen haben 
ergeben, dass diese Vernetzung in Zukunft auch österreichweit stärker ausgebaut werden muss.

Eine weitere zentrale Leistung des Nationalfonds für die Erinnerungskultur ist die Projektförderung, die von Beginn an eine Kernaufgabe dargestellt hat. Die Projektförderung wird auch in der neuen Organisationsstruktur als eigener Bereich abgebildet und soll laut Aussagen einiger befragter Personen in Zukunft noch weiter ausgebaut werden. Es werden zivilgesellschaftliche und lokale Initiativen unterstützt, die zur Bewusstseinsbildung beitragen. Das gestiegene Engagement der Zivilgesellschaft lässt sich auch durch die geförderten Projekte erkennen. Im Sinne dieser Bewusstseinsbildung hat die Projektförderung ebenfalls zu einer Veränderung der Erinnerungskultur geführt. Der Nationalfonds wird als maßgebliche Förderstelle betrachtet.

Es ist festzuhalten, dass der Nationalfonds von den Mitarbeiterlnnen trotz kritischer Äußerungen als einzigartige Organisation gesehen wird. Das Engagement der MitarbeiterInnen stellt eine ungeheure Ressource dar, die auch in Zukunft als wesentlich erachtet werden sollte. Als zentralen Beweggrund, der motivierend wirkt, nannten alle befragten Mitarbeiterlnnen die gesellschaftspolitische Verantwortungsübernahme, die diese Tätigkeit für sie bedeutet. Die unbürokratische Herangehensweise und die amikalen Umgangsformen haben den Nationalfonds zu einem geschätzten und auch außergewöhnlichen Arbeitgeber gemacht. Aus einigen Antworten hat sich induktiv ergeben, dass die Gründung des Nationalfonds als Meilenstein der österreichischen Erinnerungskultur gesehen werden kann. Einige der befragten Personen betrachten es als ein Privileg, über die Jahre hinweg mit vielen Überlebenden gearbeitet haben zu dürfen. Es wird eine Herausforderung für den Nationalfonds werden, die zukünftige Erinnerungskultur ohne Zeitzeuglnnen weiterhin lebendig zu gestalten und neue Ansätze zu finden. Es hat sich durch die Auswertung der vorliegenden Untersuchung die weiterführende Frage ergeben, welcher Platz dem Vergessen in der Erinnerungskultur gewidmet werden darf oder soll. Solange es jedoch noch Überlebende gibt, ist Erinnern und Gedenken, auch im Rahmen von institutionalisierten Gedenkveranstaltungen, ein Ausdruck des Respekts den Überlebenden gegenüber. 
So wie der erste Generationenwechsel ab Ende der 1960er Jahre einen Erfolg der opferorientierten Erinnerungskultur und eine umfassende Auseinandersetzung mit der nationalsozialistischen Vergangenheit und dem Holocaust ermöglichte, so stellt nun ein erneuter Generationenwechsel die Zukunft der Erinnerungskultur vor neue Herausforderungen. Als zentraler und essentieller Ansatz für die zukünftige Arbeit und Gestaltung von Erinnerungskultur wurde in den Interviews immer wieder die Notwendigkeit der Herstellung eines Gegenwartsbezugs genannt. In diesem Zusammenhang wurde auch angemerkt, dass der derzeitige Zugang zu Erinnerungskultur zu akademisch sei. Die Einbeziehung weiterer wissenschaftlicher Disziplinen, auch aus dem sozialwissenschaftlichen Bereich, könnte einen niederschwelligeren Zugang erleichtern und neue Zielgruppen erschließen.

Die Vermittlungsarbeit wird als eine der zentralsten zukünftigen Aufgaben des Nationalfonds wahrgenommen. Durch den Datenschatz, den das einzigartige Archiv des Nationalfonds bietet, können die lebensgeschichtlichen Erinnerungen der ZeitzeugInnen weitergegeben werden. Ein zukünftiger Zugang wäre die Weitergabe der Erinnerungen und die Erzählung über die Erfahrungen in der Arbeit mit den Überlebenden durch MitarbeiterInnen des Nationalfonds. Für nachfolgende Generationen bietet das Archiv des Nationalfonds, das auch durch die Digitalisierung einen erleichterten Zugang ermöglichen wird, eine wichtige Quelle für zukünftige Familienrecherchen und wissenschaftliche Forschungen. Technische Möglichkeiten, wie sie auch in den Befragungen genannt wurden, können in Zukunft genutzt werden, um die Thematik einem jungen Publikum zu erschließen. Ebenso wird der moralisierende Zugang überdacht werden müssen, um Abwehrhaltungen, die sich oft in der Frage „Was hat das noch mit mir zu tun?" zeigen, entgegenwirken zu können.

Bemerkenswert waren die Ergebnisse zur Frage nach zukünftigen Tätigkeitsfeldern des Nationalfonds. In den Aussagen einzelner InterviewpartnerInnen zeichnete sich der Wunsch ab, dass der Nationalfonds auch weiterhin bei der Parlamentsdirektion angesiedelt sein soll. Er wird als Kompetenzzentrum der Republik gesehen und könnte den Aussagen der befragten Expertinnen zufolge einerseits in Zukunft als Koordinierungsstelle für staatliches Erinnern und Gedenken, sowie andererseits als eine mögliche Koordinierungsstelle für ein bisher fehlendes österreichisches Holocaust- Museum fungieren. Ein Holocaust-Museum als fehlende Wirkungsstätte 
im Feld der Erinnerungskultur hat sich induktiv aus den Aussagen einiger befragter Interviewpartnerinnen ergeben. In Hinblick auf die Zukunftsperspektiven des Nationalfonds wurde in manchen Antworten allerdings auch eine Unsicherheit darüber, wie lange und in welcher Form der Nationalfonds zukünftig bestehen würde, artikuliert. Diese Unsicherheit lässt sich auf die noch nicht klar definierte Umsetzung der neuen Aufgaben durch die gesetzliche Erweiterung der Bereiche zurückführen, da die neuen Strukturen im Nationalfonds noch nicht gänzlich implementiert sind. Diese Umsetzung wird erst im 4. Quartal 2019 erfolgen und weiter ausgebaut werden.

Durch die Gesetzesnovelle aus dem Jahr 2017, die aufgrund der bevorstehenden Beendigung des Allgemeinen Entschädigungsfonds Ende 2019 und der Legitimation von Aufgaben, die bis dahin noch nicht gesetzlich verankert waren, beschlossen wurde, wurde auch der Grundstein für eine Neuorganisation des Nationalfonds gelegt. Die Formulierungen der Novelle entsprechen in ihren Grundzügen den Artikeln der Stockholm Deklaration aus dem Jahr 2000. In der Deklaration wurde die Verpflichtung zur Aufklärung über den Holocaust und zur Verankerung der Erinnerung an den Holocaust und seine Folgen im kollektiven Gedächtnis formuliert. Ebenso wurde die Verpflichtung zur Bewahrung des Gedenkens an die Opfer und die Personen, die Widerstand geleistet haben, in die Stockholm Deklaration aufgenommen. Die Forschung über den Holocaust und seine Folgen zu verstärken und die Öffnung der Archive, um einen Zugang zu Forschung zu gewährleisten, zählen ebenfalls zu den Verpflichtungen, denen die Deklaration Ausdruck verleiht. Die Stockholm Deklaration soll über das nationale Gedächtnis hinaus zur Entwicklung eines kosmopolitischen Gedächtnisses beitragen. In den Formulierungen der Stockholm Deklaration und der Gesetzesnovelle des Nationalfonds 2017 sind Parallelen zu erkennen. Die genannten Bestrebungen wurden auch in der Novellierung des NF-G formuliert.

Die Umstrukturierung des Nationalfonds und die Implementierung einer neuen Organisationsstruktur auf Basis der Gesetzesnovelle 2017 haben etliche Herausforderungen in organisatorischer Hinsicht zur Folge. Der Nationalfonds wuchs von einer kleinen Organisation mit wenigen Mitarbeiterlnnen zu einer großen Institution, die insgesamt drei Fonds umfasste und ca. 160 MitarbeiterInnen hatte, 
heran. Eine der aktuellen Herausforderungen ist es, aufgrund des Wegfalls von Arbeitsbereichen und der bevorstehenden Auflösung des Allgemeinen Entschädigungsfonds, eine verkleinerte Organisation strukturiert neu aufzubauen. Unter Einbeziehung einer externen Unternehmensberatung wurde eine neue, den aktuellen Aufgaben angepasste, Organisationsstruktur eingeführt, die auch den bildungs- und gesellschaftspolitischen Auftrag des Nationalfonds in Zukunft widerspiegelt. Der Nationalfonds wird dadurch auch offiziell von einer Auszahlungsund Förderstelle zu einer Institution für Vermittlung und Gedenken. Der Prozess ist vor allem für die MitarbeiterInnen schwierig, wie aus den Antworten in den Befragungen zu erkennen war. Für die Belegschaft bedeutet es Veränderungen in ihren Funktionen und in der Arbeit. Langsam gewachsene und in Eigeninitiative der Mitarbeiterlnnen gestaltete Strukturen wurden durch neue ersetzt, die in diesem Prozess nur teilweise mitgestaltet werden konnten. In den Befragungen der Belegschaft war zu erkennen, dass eine vermehrte inhaltliche Involvierung in den Prozess gewünscht gewesen wäre. Aus den Befragungen war klar zu erkennen, dass MitarbeiterInnen dieser Umstellung mit Skepsis, Unsicherheit und auch Unverständnis gegenüberstehen. Eine Herausforderung für die Geschäftsleitung und die neue Führungsebene wird sein, die Belegschaft proaktiv auf dem Weg in die neue Unternehmensstruktur zu begleiten und zu führen. Die Umsetzung und die inhaltliche Gestaltung der neuen Bereiche wird gelingen müssen, um den Nationalfonds für die Erfüllung seiner Aufgaben zukünftig gut positionieren zu können. Eine der zentralen Herausforderungen wird die Förderung von bereichsübergreifender Transparenz und Kommunikation innerhalb der Organisation darstellen.

Eine deutliche Erkenntnis der Ergebnisse war auch das Engagement der Mitarbeiterlnnen im Nationalfonds, ohne dem die Bewältigung der Arbeit und der sensible Zugang $\mathrm{zu}$ den AntragstellerInnen unter den zahlreichen Herausforderungen nicht in der Form gelingen hätte können. Diesem Engagement ist es auch zu verdanken, dass der Nationalfonds zu einer etablierten und anerkannten Institution geworden ist.

Eine maßgebliche, aber ungeplante Erkenntnis war die Feststellung, dass es neben den sozialtherapeutischen Angeboten anderer Einrichtungen auch direkt im 
Nationalfonds MitarbeiterInnen mit psychosozialer Ausbildung bedurft hätte. Aufgrund der Aussagen der befragten Personen wäre kritisch anzumerken, dass beim Nationalfonds seit der Dauer des Bestehens nicht explizit MitarbeiterInnen aus psychosozialen Berufen angestellt wurden. Geschulte Fachkräfte aus dem psychosozialen Bereich mit fachspezifischem Wissen und Expertisen hätten eventuellen Retraumatisierungen von Antragstellerlnnen noch besser vorbeugen und entgegenwirken und auch einen professionelleren Zugang zur eigenen Abgrenzung in Hinblick auf Psychohygiene ermöglichen können. Die Befragten, die die Anfangszeit des Nationalfonds miterlebt haben, berichteten über die starke Belastung, der die MitarbeiterInnen nicht nur durch das Ausmaß der Arbeit, sondern auch auf emotionaler Ebene ausgesetzt waren. Der Umgang mit der psychischen Belastung hätte rückblickend durch geschultes Personal besser bewältigt werden können.

Die Deutung der Kritik an der Erinnerungskultur war eine weitere unerwartete Erkenntnis. In der Fachwelt wurde Kritik an einer Ritualisierung und Institutionalisierung geäußert, wie auch im Literaturteil der vorliegenden Arbeit ausgeführt wurde. Befragungsergebnisse haben jedoch gezeigt, dass diese Kritik durchaus positiv behaftet sein kann. Kritik wird auch durch die erfolgreiche Etablierung der Erinnerungskultur in ihrer derzeitigen Erscheinungsform bedingt. Durch eine Ritualisierung kann Gedenken im kollektiven Gedächtnis verankert werden. Es soll jedoch keine inhaltslose Gedenk- und Erinnerungskultur werden, die durch leere Rituale am Leben erhalten wird. Laut Assmann entsteht das Unbehagen an der Erinnerungskultur durch die Institutionalisierung. Dem könne durch zivilgesellschaftliche Auseinandersetzung entgegengewirkt werden. Es hat sich in der Untersuchung gezeigt, dass vor allem durch die Projektförderung des Nationalfonds das angesprochene zivilgesellschaftliche Engagement unterstützt und gefördert werden kann.

In Anlehnung an die verschiedenen Gedächtnistheorien wäre zu überlegen, ob man in den heutigen heterogeneren Gesellschaften noch von einem kollektiven Gedächtnis sprechen kann oder ob man nicht Assmanns Vorschlag, stattdessen von sozialen, politischen oder nationalen Gedächtnissen zu sprechen, folgen sollte. Es stellt sich in diesem Zusammenhang aufgrund der Heterogenität der Gesellschaften 
auch die Frage, wer zukünttig Anteil an einem kollektiven Gedächtnis haben kann und wie zukünftige Generationen den Begriff des kollektiven Gedächtnisses interpretieren werden. Erinnerungskultur bezieht sich derzeit ausschließlich auf die kollektive Erinnerung an den Holocaust. Die bestehende Erinnerungskultur sollte auf eine Erinnerungskultur erweitert werden, die aktuelle Bezüge zu Flucht- und Ausgrenzungserfahrungen der heutigen heterogenen Gesellschaften einbeziehen kann.

Die Opferorientierung, die durch den Generationenwechsel den dadurch entstehenden nötigen Abstand für eine selbstkritische Reflexion erst ermöglicht hatte, ist die vorherrschende Orientierung der Erinnerungskultur. Diese Opferorientierung war ein wichtiger Schritt in der Entwicklung der Erinnerungskultur. Es steht allerdings außer Frage, dass die Erinnerungskultur in Zukunft zu aktualisieren sein wird, wie auch bereits Assmann ausgeführt hat. Neben dem Ansatz der Opferorientierung muss ein neuer Fokus auf die Entstehungsgründe von Ausgrenzungsgesellschaften gelegt und auch hier wieder auf aktuelle Entwicklungen Bezug genommen werden, wie es Welzer beschrieben hat.

In der Stockholm Deklaration wurde bereits die Verantwortung der Völkergemeinschaft, Rassismus, Ausländerfeindlichkeit und Antisemitismus entgegenzutreten, festgehalten. Dieser Absatz der Deklaration, die im Jänner 2000 verabschiedet wurde, sollte als der geforderte Ansatz einer Erweiterung der Erinnerungskultur interpretiert werden und als Richtlinie für die Umsetzung einer solchen dienen. Erinnerungskultur sollte sich in Zukunft nicht mehr nur ausschließlich auf die Geschichte des Holocaust beziehen, sondern aktuelle Bezugsrahmen schaffen, die näher an der Lebenswelt der heutigen Jugend liegen, da die generationale Verbindung zu den Geschehnissen von damals langsam abnimmt. Laut Welzer stellen Jugendliche mit Migrationshintergrund eine neue Zielgruppe dar. Der Nationalfonds kann hier nicht nur im Rahmen der Vermittlungsarbeit an Schulen, sondern auch durch zukünftige Kooperationen mit Einrichtungen im sozialen Bereich und einer niederschwelligen Herangehensweise diese aktuellen Bezugsrahmen schaffen. Die Ergebnisse der Studie der Claims Conference haben die Notwendigkeit einer neuen Ausrichtung der Erinnerungskultur gezeigt. Eine Erweiterung des Feldes der sich mit Erinnerungskultur beschäftigenden Wissenschaften um 
Sozialarbeitswissenschaft und weiterer Bezugsdisziplinen kann hier einen neuen Ansatz darstellen.

Knigge und Frei halten fest: „Die Zukunft der Erinnerung lässt sich bedenken, kaum aber festschreiben." (Knigge, Frei, 2002, S.XI) Durch die vielfältigen Aufgabenbereiche des Nationalfonds und eine zukunftsorientierte und offene Herangehensweise kann es gelingen, neue Ansätze für die Erinnerungskultur zu kreieren und $z u$ einer begrifflichen Erweiterung im Sinne eines interkulturellen Verständnisses und einer gesellschafts- und bildungspolitischen Instanz gegen Antisemitismus, Rassismus und Ausgrenzung beizutragen. Selbst wenn sich die Zukunft der Erinnerung nicht festschreiben lässt, so kann sie sich doch nicht nur bedenken, sondern auch zukunftsgerichtet neu gestalten lassen. 


\section{Literaturverzeichnis}

Assmann, A. (2016a). Das neue Unbehagen an der Erinnerungskultur. Eine Intervention. München: C.H. Beck.

Assmann, A. (2016b). Zur Kritik, Karriere und Relevanz des Gedächtnisbegriffs. Die ethische Wende in der Erinnerungskultur. In L. Radonić, H. Uhl, (Hg.) (2016). Gedächtnis im 21. Jahrhundert: Zur Neuverhandlung eines kulturwissenschaftlichen Leitbegriffs (S.29-42). Bielefeld: Transcript Verlag.

Assmann, A. (2018). Der lange Schatten der Vergangenheit. Erinnerungskultur und Geschichtspolitik. München: C.H. Beck.

Berger, P.L., Luckmann, T. (2018). Die gesellschaftliche Konstruktion der Wirklichkeit. Frankfurt am Main: Fischer Verlag.

Brisch, K.H. (2013). Die Weitergabe von traumatischen Erfahrungen von Bindungspersonen an die Kinder. In M. Rauwald (Hg.). Vererbte Wunden. Transgenerationale Weitergabe traumatischer Erfahrungen (S.38-46). Weinheim, Basel: Beltz Verlag.

Dömötör M., Höller H., Keglovits B, Killic J., Mayerhofer S., (2013). „Sozialökonomische Betriebe als Chance auf nachhaltige Integration in den ersten Arbeitsmarkt am Beispiel von „fix und fertig“".. Bedeutung von Multiproblemlagen und Beurteilung von Wiedereingliederungsmaßnahmen aus Sicht der KlientInnen und ExpertInnen. Unveröffentlichte Bachelorarbeit. Wien: FH Campus Wien.

Düringer, H. (2012). Die Vergangenheit ist nicht abgeschlossen. Religiöse Aspekte des Erinnerns. In M. Frölich, U. Jureit, C. Schneider (Hrsg.) (2012). Das Unbehagen an der Erinnerung - Wandlungsprozesse im Gedenken an den Holocaust (S.55-66). Frankfurt am Main: Brandes \& Apsel Verlag GmbH.

Frei, N. (2002). Die Zukunft der Erinnerung. Geschichtswissenschaft, Gedenkstätten, Medien. In In V. Knigge, N. Frei. (Hrsg.) Verbrechen erinnern. Die Auseinandersetzung mit Holocaust und Völkermord (S.369-377). München: Verlag C.H. Beck.

Froschauer, U., Lueger, M. (2003). Das qualitative Interview. Zur Praxis interpretativer Analyse sozialer Systeme. Wien

Frölich, M., Jureit, U., Schneider, C., (Hrsg.) (2012). Das Unbehagen an der ErinnerungWandlungsprozesse im Gedenken an den Holocaust. Frankfurt am Main: Brandes \& Apsel Verlag $\mathrm{GmbH}$.

Gläser, J., Laudel, G. (2010). Experteninterviews und qualitative Inhaltsanalyse. Wiesbaden: VS Verlag für Sozialwissenschaften.

Grandsard, C. (2011). Singularität vs. Universalität der Shoah - Implikationen für die Psychotherapie. In J. Brunner, N. Zajde (Hg). Tel Aviver Jahrbuch für deutsche Geschichte 39: Holocaust und Trauma. Kritische Perspektiven zur Entstehung und Wirkung eines Paradigmas (S.209-222). Göttingen: Wallstein Verlag.

Halbwachs, M. (1985). Das Gedächtnis und seine sozialen Bedingungen. Suhrkamp Taschenbuch Wissenschaft. Berlin: Suhrkamp. 
Jureit, U. (2012). Normative Verunsicherungen. Die Besichtigung einer erinnerungspolitischen Zäsur. In Frölich, M., Jureit, U., Schneider, C., (Hrsg.). Das Unbehagen an der Erinnerung - Wandlungsprozesse im Gedenken an den Holocaust (S.2136). Frankfurt am Main: Brandes \& Apsel Verlag GmbH.

Kaminer-Zamberk, E.I. (2013). Die Folgen der Shoah in der Zweiten Generation. In M. Rauwald (Hg.). Vererbte Wunden. Transgenerationale Weitergabe traumatischer Erfahrungen (S.77-88). Weinheim, Basel: Beltz Verlag.

Kellermann, N.P.F. (2011). „Geerbtes Trauma“ - die Konzeptualisierung der transgenerationellen Weitergabe von Traumata. In J. Brunner, N. Zajde (Hg). Tel Aviver Jahrbuch für deutsche Geschichte 39: Holocaust und Trauma. Kritische Perspektiven zur Entstehung und Wirkung eines Paradigmas (S.137-160). Göttingen: Wallstein Verlag.

Klüger, R. (2008). weiter leben. Eine Jugend. Wien: Echomedia Verlag Ges.m.b.h.

Knigge, V. (2002). Gedenkstätten und Museen. In V. Knigge, N. Frei. (Hg.). Verbrechen erinnern. Die Auseinandersetzung mit Holocaust und Völkermord (S.378-389). München: Verlag C.H. Beck.

Knigge, V., Frei, N. (2002) (Hg.). Verbrechen erinnern. Die Auseinandersetzung mit Holocaust und Völkermord. München: Verlag C.H. Beck.

Knight, R. (2000). „Ich bin dafür, die Sache in die Länge zu ziehen.“ Wortprotokolle der österreichischen Bundesregierung von 1945-1952 über die Entschädigung der Juden. Wien: Böhlau Verlag

Kroh, J. (2012). Das erweiterte Europa auf dem Weg zu einem gemeinsamen Gedächtnis? Die Stockholmer „Holocaust-Konferenz“ und ihre Bedeutung für die europäische Erinnerung. In Frölich, M., Jureit, U., Schneider, C., (Hrsg.). Das Unbehagen an der Erinnerung Wandlungsprozesse im Gedenken an den Holocaust (S.201-216). Frankfurt am Main: Brandes \& Apsel Verlag GmbH.

Lessing, H. (2000). Einblicke und Ausblicke. Der Nationalfonds der Republik Österreich für Opfer des Nationalsozialismus. Gesetzliche Grundlagen und Intentionen. In Nationalfonds der Republik Österreich für Opfer des Nationalsozialismus (Hg.). In die Tiefe geblickt. Lebensgeschichten (S.67-73). Wien: Edition INW, Illustrierte Neue Welt.

Levy, D., Sznaider, N. (2007). Erinnerung im globalen Zeitalter: Der Holocaust. Frankfurt am Main: Suhrkamp Verlag.

Leys, R. (2011). Die „Überlebensschuld“ im psychoanalytischen Diskurs - Ein kurzer historischer Überblick. In J. Brunner, N. Zajde (Hg). Tel Aviver Jahrbuch für deutsche Geschichte 39: Holocaust und Trauma. Kritische Perspektiven zur Entstehung und Wirkung eines Paradigmas (S.86-115). Göttingen: Wallstein Verlag.

Mayerhofer, S. (2014). Das Holocaust-Syndrom als Form der posttraumatischen Belastungsstörung. Die Handlungsmöglichkeiten Sozialer Arbeit in der psychosozialen Betreuung von Child Survivors am Beispiel von ESRA. Unveröffentlichte Bachelorarbeit. Wien: FH Campus Wien.

Mayring, P. (2002). Einführung in die qualitative Sozialforschung. Eine Anleitung zu qualitativem Denken. Weinheim und Basel: Beltz Verlag.

Mayring, P. (2015). Qualitative Inhaltsanalyse. Grundlagen und Techniken. Weinheim und Basel: Beltz Verlag. 
Meier, C. (2010). Das Gebot zu vergessen und die Unabweisbarkeit des Erinnerns. Vom öffentlichen Umgang mit schlimmer Vergangenheit. München: Siedler Verlag.

Pelinka, A. (2001). Zum Geleit. In A. Sternfeld. Betrifft: Österreich. Von Österreich betroffen. (S.11-19). Wien: Böhlau Verlag.

Perz, B. (2002). Österreich. In V. Knigge, N. Frei. Verbrechen erinnern. Die Auseinandersetzung mit Holocaust und Völkermord (S.150-162). München: Verlag C.H. Beck.

Radonić, L., Uhl, H. (Hg.) (2016). Gedächtnis im 21. Jahrhundert: Zur Neuverhandlung eines kulturwissenschaftlichen Leitbegriffs. Bielefeld: Transcript Verlag.

Rauwald, M., Maccarrone Erhardt, R. (2013). Therapeutische Herausforderungen bei der Behandlung von transgenerational vermittelten Traumata. In M. Rauwald (Hg.). Vererbte Wunden. Transgenerationale Weitergabe traumatischer Erfahrungen (S.57-65). Weinheim, Basel: Beltz Verlag.

Sabrow, M. (2012a). Held und Opfer. Zum Subjektwechsel deutscher Vergangenheitsbewältigung im 20. Jahrhundert. In Frölich, M., Jureit, U., Schneider, C., (Hrsg.) (2012). Das Unbehagen an der Erinnerung - Wandlungsprozesse im Gedenken an den Holocaust. (S.37-54). Frankfurt am Main: Brandes \& Apsel Verlag GmbH.

Sabrow, M., Frei, N. (Hrsg.) (2012b). Die Geburt des Zeitzeugen nach 1945. Göttingen: Wallstein Verlag.

Sänger, R., Udolf, M. (2013). Transgenerationale Traumaweitergabe im Kinder- und Jugendhilfesystem - Auswirkungen und Umgang. In M. Rauwald (Hg.). Vererbte Wunden. Transgenerationale Weitergabe traumatischer Erfahrungen (S.139-148). Weinheim, Basel: Beltz Verlag.

Schneider, C. (2012). Generation im Abtritt. Vom Schicksal historischer Gegenidentifizierungen. In Frölich, M., Jureit, U., Schneider, C., (Hrsg.). Das Unbehagen an der Erinnerung - Wandlungsprozesse im Gedenken an den Holocaust (S.85-100). Frankfurt am Main: Brandes \& Apsel Verlag GmbH.

Schrader, C. (2013). „Diesen Deckel wollte ich eigentlich gar nicht aufmachen“ - zur Reaktivierung transgenerationaler Traumata im Alter. In M. Rauwald (Hg.). Vererbte Wunden. Transgenerationale Weitergabe traumatischer Erfahrungen (S.38-46). Weinheim, Basel: Beltz Verlag.

Sternfeld, A. (1990). Betrifft: Österreich. Wien: Löcker Verlag.

Sternfeld, A. (2001). Betrifft: Österreich. Von Österreich betroffen. Wien: Böhlau Verlag Ges. m. b. H. und Co. KG.

Uhl, H. (2012). Vom Pathos des Widerstands zur Aura des Authentischen. Die Entdeckung des Zeitzeugen als Epochenschwelle der Erinnerung. In M. Sabrow, N. Frei (Hrsg.) (2012b). Die Geburt des Zeitzeugen nach 1945 (S.224-246). Göttingen: Wallstein Verlag.

Unfried, N. (2013). Biologische und neurobiologische Hintergründe der Traumatisierung. In M. Rauwald (Hg.). Vererbte Wunden. Transgenerationale Weitergabe traumatischer Erfahrungen (S.47-54). Weinheim, Basel: Beltz Verlag. 
Vyssoki, D., Tauber, T., Strusievici, S., Schürmann-Emanuely, A. (2004). Trauma bei den Opfern der NS-Verfolgung. In A. Friedmann, P. Hofmann, B. Lueger-Schuster, M. Steinbauer, D. Vyssoki (Hg.). Psychotrauma. die Posttraumatische Belastungsstörung (S.197-211). Wien: Springer Verlag.

Welzer, H. (2002). Der Holocaust im deutschen Familiengedächtnis. In V. Knigge, N. Frei. Verbrechen erinnern. Die Auseinandersetzung mit Holocaust und Völkermord (S.342-358). München: Verlag C.H. Beck.

Welzer, H, (2012). Vom Zeit- zum Zukunftszeugen. Vorschläge zur Modernisierung der Erinnerungskultur. In M. Sabrow, N. Frei (Hrsg.) (2012b). Die Geburt des Zeitzeugen nach 1945 (S.33-48). Göttingen: Wallstein Verlag.

Zajde, N. (2011). Die Shoah als Paradigma des psychischen Traumas. In J. Brunner, N. Zajde (Hg). Tel Aviver Jahrbuch für deutsche Geschichte 39: Holocaust und Trauma. Kritische Perspektiven zur Entstehung und Wirkung eines Paradigmas (S.17-39). Göttingen: Wallstein Verlag.

\section{Internetquellen:}

Achleitner, J. (2016). 1986 - ein Jahr mit Folgen. URL:

https://www.nachrichten.at/nachrichten/thema/1986-Ein-Jahr-mit-Folgen;art180211,2320366 [Datum des Zugriffs: 30.8.2019]

Allgemeiner Entschädigungsfonds für Opfer des Nationalsozialismus (o.V.) (o.J.).

Naturalrestitution. URL: https://www.entschaedigungsfonds.org/naturalrestitution.html [Datum des Zugriffs: 30.8.2019]

Allgemeiner Entschädigungsfonds für Opfer des Nationalsozialismus (o.V.) (o.J.). Organe des Allgemeinen Entschädigungsfonds. URL:

https://www.entschaedigungsfonds.org/organe.html [Datum des Zugriffs: 30.8.2019]

Allgemeiner Entschädigungsfonds für Opfer des Nationalsozialismus (o.V.) (o.J.). Rechtsgrundlagen. URL: https://www.entschaedigungsfonds.org/gesetze.html [Datum des Zugriffs: 30.8.2019]

Allgemeiner Entschädigungsfonds für Opfer des Nationalsozialismus (o.V.) (o.J.). Über uns. URL: https://www.entschaedigungsfonds.org/ueber-uns.html [Datum des Zugriffs: 30.8.2019]

Allgemeiner Entschädigungsfonds für Opfer des Nationalsozialismus (o.V.) (o.J.). Verfahren vor dem Antragskomitee. URL: https://www.entschaedigungsfonds.org/verfahren-vor-demantragskomitee.html [Datum des Zugriffs: 30.8.2019]

Allgemeiner Entschädigungsfonds für Opfer des Nationalsozialismus (o.V.) (o.J.). Verfahren vor der Schiedsinstanz. URL: https://www.entschaedigungsfonds.org/verfahren-vor-derschiedsinstanz.html [Datum des Zugriffs: 30.8.2019]

Allgemeiner Entschädigungsfonds für Opfer des Nationalsozialismus (o.V.) (o.J.). Vermögensentschädigung. URL: https://www.entschaedigungsfonds.org/vermoegensentschaedigung.html [Datum des Zugriffs: 30.8.2019]

Allgemeiner Entschädigungsfonds für Opfer des Nationalsozialismus (o.V.) (2018). Schiedsinstanz für Naturalrestitution: Antragsbearbeitung abgeschlossen. URL: https://www.entschaedigungsfonds.org/meldung/schiedsinstanz-fuer-naturalrestitutionantragsbearbeitung-abgeschlossen.html [Datum des Zugriffs: 30.8.2019] 
Allgemeiner Entschädigungsfonds für Opfer des Nationalsozialismus (o.V.) (2019).

Newsletter Juni 2019. URL:https://www.entschaedigungsfonds.org/meldung/newsletter-juni2019.html [Datum des Zugriffs: 30.8.2019]

Allgemeiner Entschädigungsfonds für Opfer des Nationalsozialismus (o.V.) (2019). Rund 215 Millionen US-Dollar an rund 25.000 Berechtigte ausbezahlt. URL:

https://www.entschaedigungsfonds.org/meldung/rund-215-millionen-us-dollar-an-rund-25000-berechtigte-ausbezahlt.html [Datum des Zugriffs: 30.8.2019]

Angerer, C. (o.J.). Wozu brauchen wir NS-Gedenkstätten? Und warum ist diese Frage nicht verkehrt? URL: https://www.ph-online.ac.at/ph-

ooe/voe main2.getVollText?pDocumentNr=484712\&pCurrPk=5524

[Datum des Zugriffs: 30.8.2019]

Assmann, J. (o.J.) Kollektives Gedächtnis und kulturelle Identität. URL:

https://core.ac.uk/download/pdf/35125036.pdf [Datum des Zugriffs: 30.8.2019]

Assmann, A. (2007). Die Last der Vergangenheit. URL: https://zeithistorische-

forschungen.de/3-2007/id\%3D4398 [Datum des Zugriffs: 30.8.2019]

Blum, J. (2012). Holocaustgedenken: Was gesagt werden muss. URL:

http://www.hagalil.com/2012/07/yehuda-blum/ [Datum des Zugriffs: 19.11.2018]

Bruckmüller, E. (2011). Kollektives Gedächtnis und öffentliches Gedenken.

URL: https://www.doew.at/cms/download/cri8a/gum bruckmueller.pdf [Datum des Zugriffs:

30.8.2019]

Bundesministerium für Arbeit, Soziales, Gesundheit und Konsumentenschutz. Internationale statistische Klassifikation der Krankheiten und verwandter Gesundheitsprobleme. 10.

Revision - BMGF-Version 2017.

URL:https://www.sozialministerium.at/cms/site/attachments/7/7/0/CH3987/CMS1537963053

638/icd-10 bmgf 2017+ - systematisches verzeichnis.pdf [Datum des Zugriffs: 30.8.2019]

Bundesministerium für auswärtige Angelegenheiten (Hg.) (2001): Außenpolitische

Dokumentation. Sonderdruck. Osterreichische Maßnahmen zur Restitution und

Entschädigung von Opfern des Nationalsozialismus. Wien. URL:

https://www.nationalfonds.org/files/content/documents/nf/UBl16A016990.pdf [Datum des

Zugriffs: 30.8.2019]

Claims Conference. The Conference on Jewish Material Claims Against Germany. (2019).

Neue Erhebung der Claims Conference stellt kritische Lücken bei Holocaust-Kenntnissen in Österreich fest. URL: https://www.claimscon.de/no cache/presse-

news/aktuelles/newsdetail.html?tx news pi1\%5Bnews\%5D=58\&tx news pi1\%5Bcontroller $\% 5 \mathrm{D}=$ News\&tx news pi $\% 5 \mathrm{Baction} \% 5 \mathrm{D}=$ detail\&cHash=4f7002a76dc756505ce0173a66e38 a42 [Datum des Zugriffs: 30.8.2019]

Corneließen, C. (2008). Erinnern in Europa. URL:

http://www.bpb.de/geschichte/zeitgeschichte/geschichte-und-erinnerung/39860/erinnern-ineuropa? $\mathrm{p}=$ all [Datum des Zugriffs: 30.8.2019]

Demokratiezentrum Wien. (o.V.) (2014). Waldheim-Debatte.

URL: http://www.demokratiezentrum.org/wissen/wissensstationen/waldheim-debatte.html

[Datum des Zugriffs: 30.8.2019] 
Die Presse (o.V.) (o.J.). Jahrestag: Waldheim, die SA, sein Pferd und die ,Pflicht'. URL: https://diepresse.com/home/zeitgeschichte/1342242/Jahrestag Waldheim-die-SA-seinPferd-und-die-Pflicht\#slide-1342242-9 [Datum des Zugriffs: 30.8.2019]

Dokumentationsarchiv des österreichischen Widerstandes. (o.V.) (o.J.) Rückstellungen entzogener Vermögen. URL: http://ausstellung.de.doew.at/b39.html [Datum des Zugriffs: 1.9.2019]

erinnern.at (o.V.) (o.J.) 27. Jänner: Internationaler Holocaust-Gedenktag. URL: http://www.erinnern.at/bundeslaender/oesterreich/gedenktage/27.-jaenner/27.-jaennerinternationaler-holocaust-gedenktag [Datum des Zugriffs: 30.8.2019]

erinnern.at (o.V.) (o.J.). Was ist Antisemitismus? Österreich nimmt IHRA-Arbeitsdefinition von Antisemitismus an. URL:

http://www.erinnern.at/bundeslaender/oesterreich/e bibliothek/antisemitismus-1/was-istantisemitismus-oesterreich-nimmt-ihra-arbeitsdefinition-von-antisemitismus-an [Datum des Zugriffs: 30.8.2019]

Findbuch für Opfer des Nationalsozialismus (o.V.) (o.J.). Über uns. URL: https://www.findbuch.at/ueber-uns.html [Datum des Zugriffs: 30.8.2019]

Findbuch für Opfer des Nationalsozialismus (o.V.) (o.J.). Das Findbuch. URL: https://www.findbuch.at/das-findbuch.html [Datum des Zugriffs: 30.8.2019]

Fonds zur Instandsetzung der jüdischen Friedhöfe in Österreich. (o.V.) (o.J.) Jüdische Friedhöfe. Über uns. URL: https://www.friedhofsfonds.org/ueber-uns.html [Datum des Zugriffs: 30.8.2019]

Fonds zur Instandsetzung der jüdischen Friedhöfe in Österreich. (o.V.) (o.J.) Organe des Friedhofsfonds. Beirat. URL: https://www.friedhofsfonds.org/organe/beirat.html\#beirat [Datum des Zugriffs: 30.8.2019]

Fonds zur Instandsetzung der jüdischen Friedhöfe in Österreich. (o.V.) (o.J.)

Rechtsgrundlagen. URL: https://www.friedhofsfonds.org/gesetze.html [Datum des Zugriffs: 30.8.2019]

Frank, B. (2019). Als eine Fernsehserie Schockwellen auslöste. URL:

https://www.sueddeutsche.de/medien/fernsehen-holocaust-wdr-1.4276735 [Datum des Zugriffs: 30.8.2019]

Friedman, David. (1980). A Dream Fulfilled: World Gathering of Holocaust Survivors in Israel June 15-18, 1981. URL: https://www.jta.org/1980/02/14/archive/a-dream-fulfilled-worldgathering-of-holocaust-survivors-in-israel-june-15-18-1981 [Datum des Zugriffs: 30.8.2019]

Funk, M. (2018). Erinnern kann auch cool sein. URL:

https://www.zeit.de/freitext/2018/01/26/holocaust-ns-zeit-erinnerungskultur/ [Datum des Zugriffs: 30.8.2019]

Gebhardt, R. (2012). „Der Broder darf das!“ URL: http://buecher.hagalil.com/2012/03/broder3/ [Datum des Zugriffs: 30.8.2019]

Haus der Geschichte Österreich (o.V.) (o.J.) Displaced Persons. URL: https://www.hdgoe.at/displaced-persons [Datum des Zugriffs: 30.8.2019] 
Held, M. (2015). „Der Holocaust ist nicht mehr exklusiv“. URL:

https://www.t-online.de/nachrichten/deutschland/gesellschaft/id 76028134/9-11-in-

deutschland-der-holocaust-ist-nicht-mehr-exklusiv-.html [Datum des Zugriffs: 30.8.2019]

Heubner, C. (2017). Wir dürfen Auschwitz nicht den Neurechten überlassen. URL:

https://www.tagesspiegel.de/politik/gedenkkultur-und-rassismus-wir-duerfen-auschwitz-nichtden-neurechten-ueberlassen/20497244.html [Datum des Zugriffs: 30.8.2019]

International Holocaust Remembrance Alliance (o.V.) (o.J.). Arbeitsdefinition von Antisemitismus. URL: https://www.holocaustremembrance.com/de/node/196 [Datum des Zugriffs: 30.8.2019]

International Holocaust Remembrance Alliance (o.V.) (o.J.). Arbeitsdefinition von Leugnung und Verharmlosung des Holocaust URL: https://holocaustremembrance.com/de/node/122 [Datum des Zugriffs: 30.8.2019]

International Holocaust Remembrance Alliance (o.V.) (o.J.). Austria. URL:

https://www.holocaustremembrance.com/member-countries/austria [Datum des Zugriffs: 30.8.2019]

International Holocaust Remembrance Alliance (o.V.) (2019). Committee on Antisemitism and Holocaust Denial Paper on Holocaust Distortion and Denial. URL:

https://holocaustremembrance.com/sites/default/files/inline-

files/Paper\%20on\%20Distortion 0.pdf [Datum des Zugriffs: 30.8.2019]

International Holocaust Remembrance Alliance (o.V.) (o.J.). Our Structure. URL:

https://www.holocaustremembrance.com/our-structure [Datum des Zugriffs: 30.8.2019]

International Holocaust Remembrance Alliance (o.V.) (o.J.). Permanent Office. URL:

https://www.holocaustremembrance.com/index.php/permanent-office [Datum des Zugriffs: 30.8.2019]

International Holocaust Remembrance Alliance (o.V.) (o.J.) Stockholm Declaration. A commitment shared by 32 member countries. URL:

https://www.holocaustremembrance.com/index.php/de/node/17?usergroup $=7$ [Datum des Zugriffs: 30.8.2019]

Klüger, R. (2011). Gedenktag - Ruth Klügers Gedicht aus Auschwitz. URL:

https://www.parlament.gv.at/ZUSD/PDF/Gedenktagsrede RuthKlueger.pdf

[Datum des Zugriffs: 30.8.2019]

Koch, S. (2018). Der Holocaust? „Krass, so krass“ URL:

http://www.haz.de/Sonntag/Top-Thema/Der-Holocaust-Krass-so-krass [Datum des Zugriffs: 30.8.2019]

Köhler, M. (2012). Kritik der deutschen Erinnerungskultur: Ritualisiertes Gedenken. URL: http://www.hagalil.com/2012/03/schoah-11/ [Datum des Zugriffs: 30.8.2019]

Konrad, H. (o.J.). Moskauer Deklaration. URL: https://www.hdgoe.at/moskauer-deklaration [Datum des Zugriffs: 30.8.2019]

Kuretsidis-Haider, C. (o.J.) Gedenken und Mahnen. NS-Herrschaft, Erinnerungskulturen und Gedächtnislandschaften nach 1945. URL:

http://www.niemals-vergessen.at/html/nv erinnerung.html [Datum des Zugriffs: 30.8.2019] 
Lackner, H. (2016). Der Fall Kurt Waldheim: Pflicht und Dunkel. URL:

https://www.profil.at/oesterreich/history/fall-kurt-waldheim-pflicht-dunkel-6253333 [Datum des Zugriffs: 30.8.2019]

Mantler, A. (2018). „Gedächtnis und Generationserfahrung“. Zum Gedenk- und Bedenkjahr 2018 macht sich die Historikerin Heidemarie UhI Gedanken. URL:

https://www.schule.at/fileadmin/DAM/Gegenstandsportale/Politische Bildung/Dateien/Heide marie Uhl ueber das Gedenkjahr 2018.pdf [Datum des Zugriffs: 30.8.2019]

Meissner, R.S. im Auftrag des Nationalfonds (Hg.) (2005a). 10 Jahre Nationalfonds. Zahlen. Daten. Fakten. Nationalfonds der Republik Österreich für Opfer des Nationalsozialismus. Allgemeiner Entschädigungsfonds für Opfer des Nationalsozialismus. Wien. URL: https://www.nationalfonds.org/files/content/documents/nf/10 nf info de.pdf [Datum des Zugriffs: 30.8.2019]

Meissner, R.S. im Auftrag des Nationalfonds (Hg.).(2005b). 10 Jahre Nationalfonds. Einblicke. Ausblicke. Nationalfonds der Republik Österreich für Opfer des Nationalsozialismus. Wien. URL: https://www.nationalfonds.org/files/content/documents/nf/10 nf ea de.pdf [Datum des Zugriffs: 30.8.2019]

Meissner, R.S. im Auftrag des Nationalfonds (Hg.) (2015). 20 Jahre Nationalfonds der Republik Österreich für Opfer des Nationalsozialismus. Wien. URL:

https://www.nationalfonds.org/files/content/documents/nf/20-jahre-nf magazin.pdf [Datum des Zugriffs: 30.8.2019]

Mund, Heike (2019). Wie der Begriff "Holocaust" überhaupt nach Deutschland kam. URL: https://www.dw.com/de/wie-der-begriff-holocaust-\%C3\%BCberhaupt-nach-deutschlandkam/a-47254116 [Datum des Zugriffs: 30.8.2019]

Nationalfonds der Republik Österreich für Opfer des Nationalsozialismus (o.V.) (o.J.). Anerkennung verschiedener Opfergruppen.

URL:https://www.nationalfonds.org/opferanerkennung.html [Datum des Zugriffs: 30.8.2019]

Nationalfonds der Republik Österreich für Opfer des Nationalsozialismus (o.V.) (o.J.).

Aufgaben im Bereich der Kunstrestitution. URL: https://www.nationalfonds.org/verwertungerbloser-kunstgegenstaende.html [Datum des Zugriffs: 30.8.2019]

Nationalfonds der Republik Österreich für Opfer des Nationalsozialismus (o.V.) (o.J.). Die österreichische Länderausstellung aus dem Jahr 1978. URL:

https://www.nationalfonds.org/ausstellung-1978.html [Datum des Zugriffs: 30.8.2019]

Nationalfonds der Republik Österreich für Opfer des Nationalsozialismus (o.V.) (o.J.). Individualzahlungen des Nationalfonds im Überblick. URL:

https://www.nationalfonds.org/individualzahlungen-des-nationalfonds.html [Datum des Zugriffs: 30.8.2019]

Nationalfonds der Republik Österreich für Opfer des Nationalsozialismus (o.V.) (o.J.). International Holocaust Remembrance Alliance. URL: https://www.nationalfonds.org/ihra.html [Datum des Zugriffs: 30.8.2019]

Nationalfonds der Republik Österreich für Opfer des Nationalsozialismus (o.V.) (o.J.). Kuratorium. URL: https://www.nationalfonds.org/organe/kuratorium.html\#kuratorium [Datum des Zugriffs: 30.8.2019] 
Nationalfonds der Republik Österreich für Opfer des Nationalsozialismus (o.V.) (o.J.). Lebensgeschichtliche Erinnerungen. URL:

https://www.nationalfonds.org/lebensgeschichten.html [Datum des Zugriffs: 30.8.2019]

Nationalfonds der Republik Österreich für Opfer des Nationalsozialismus (o.V.) (o.J.). Mietrechtsentschädigung. URL:

https://www.nationalfonds.org/mietrechtsentschaedigung.html [Datum des Zugriffs:

30.8.2019]

Nationalfonds der Republik Österreich für Opfer des Nationalsozialismus (o.V.) (o.J.).

Projektförderung des Nationalfonds.

URL:https://www.nationalfonds.org/projektfoerderung.html [Datum des Zugriffs: 30.8.2019]

Nationalfonds der Republik Österreich für Opfer des Nationalsozialismus (o.V.) (o.J.).

Projektinformation. Beschluss zur Neugestaltung. URL:

https://www.nationalfonds.org/projektinfo.html [Datum des Zugriffs: 30.8.2019]

Nationalfonds der Republik Österreich für Opfer des Nationalsozialismus (o.V.) (o.J.). Sanierung des ehemaligen Häftlingsblocks. URL:

https://www.nationalfonds.org/sanierung.html [Datum des Zugriffs: 30.8.2019]

Nationalfonds der Republik Österreich für Opfer des Nationalsozialismus (o.V.) (o.J.). Team des Nationalfonds. URL: https://www.nationalfonds.org/team.html [Datum des Zugriffs:

30.8.2019]

Nationalfonds der Republik Österreich für Opfer des Nationalsozialismus (o.V.) (o.J.). Weitere Rechtsquellen und parlamentarische Materialien. Rechtsquellen seit Mai 1945.

URL:https://www.nationalfonds.org/weitere-rechtsquellen-und-parlamentarische-materialien [Datum des Zugriffs: 1.9.2019]

Nationalfonds der Republik Österreich für Opfer des Nationalsozialismus (Hg.) (o.V.) (2019). Media Information. June 2019. URL:

https://www.entschaedigungsfonds.org/files/content/documents/nf/Medieninformation EN 18 062019 web.pdf [Datum des Zugriffs: 30.8.2019]

Online Focus (o.V.) (2012). Henryk M. Broder zu KZ - Tourismus. „Auschwitz ist ein

Disneyland des Todes“. URL: https://www.focus.de/politik/deutschland/henry-m-broder-

kritisiert-vergangenheitsbewaeltigung-auschwitz-ist-ein-disneyland-des-

todes aid 722898.html [Datum des Zugriffs: 30.8.2019]

Rauscher, H. (2016). „Ich habe im Krieg nichts anderes getan als meine Pflicht erfüllt." URL: https://derstandard.at/2000031874110/lch-habe-im-Krieg-nichts-anderes-getan-als-meinePflicht [Datum des Zugriffs: 30.8.2019]

Rechtsinformationssystem des Bundes (o.V.) (1995). Bundesgesetzblatt für die Republik Österreich. Bundesgesetz über den Nationalfonds der Republik Österreich für Opfer des Nationalsozialismus. URL:

https://www.ris.bka.gv.at/Dokumente/BgblPdf/1995 432 0/1995 $432 \quad$ 0.pdf [Datum des Zugriffs: 30.8.2019]

Rechtsinformationssystem des Bundes (o.V.) (2017). Bundesgesetzblatt für die Republik Österreich. Bundesgesetz, mit dem das Bundesgesetz über den Nationalfonds der Republik Österreich für Opfer des Nationalsozialismus geändert wird. URL:

https://www.ris.bka.gv.at/Dokumente/BgblAuth/BGBLA 2017 | 143/BGBLA 2017 | 143.pdf sig [Datum des Zugriffs: 30.8.2019] 
Rechtsinformationssystem des Bundes (o.V.) (2019). Bundesrecht konsolidiert: Gesamte Rechtsvorschrift für Kunstrückgabegesetz, Fassung vom 27.07.2019. URL:

https://www.ris.bka.gv.at/GeltendeFassung.wxe?Abfrage=Bundesnormen\&Gesetzesnummer $=10010094$ [Datum des Zugriffs: 30.8.2019]

Reinecke, S. (2019). US-Fernsehserie „Holocaust. “Ein Meilenstein der Erinnerungskultur. URL: https://www.deutschlandfunkkultur.de/us-fernsehserie-holocaust-ein-meilensteinder.1005.de.html?dram:article id=437527 [Datum des Zugriffs: 30.8.2019]

Republik Österreich. Parlament. (o.V.) (o.J.). Biographie Dr. Kurt Waldheim. URL: https://www.parlament.gv.at/WWER/PAD 13961/ [Datum des Zugriffs: 30.8.2019]

Republik Österreich. Parlament. (o.V.) (1991). Stenographisches Protokoll der 35. Sitzung des Nationalrates der Republik Österreich. XVIII. Gesetzgebungsperiode. (S.12-16). URL: https://www.parlament.gv.at/PAKT/VHG/XVIII/NRSITZ/NRSITZ 00035/imfname 142026.pdf [Datum des Zugriffs: 30.8.2019]

Republik Österreich. Parlament. (o.V.) (1994). Stenographisches Protokoll der 173. Sitzung des Nationalrates der Republik Österreich. XVIII. Gesetzgebungsperiode. (S.63-70). URL: https://www.parlament.gv.at/PAKT/VHG/XVIII/NRSITZ/NRSITZ 00173/imfname 142596.pdf [Datum des Zugriffs: 30.8.2019]

Republik Österreich. Parlament. (o.V.) (1995). Stenographisches Protokoll der 40. Sitzung des Nationalrates der Republik Österreich. XIX. Gesetzgebungsperiode. (S.71). URL: https://www.parlament.gv.at/PAKT/VHG/XIX/NRSITZ/NRSITZ 00040/imfname 141767.pdf [Datum des Zugriffs: 30.8.2019]

Schrörs, T. (2019). Die Erschütterung. URL:

https://www.faz.net/aktuell/feuilleton/medien/serien/ard-sender-wiederholen-dieamerikanische-serie-holocaust-15972921.html [Datum des Zugriffs: 30.8.2019]

Schuller, M. (2012). Vergiss nicht. Vergesst. Der letzte Zeuge: Harald Welzer plädiert für eine neue deutsche Erinnerungskultur. URL: https://www.tagesspiegel.de/kultur/vergissnicht-vergesst/7113068.html [Datum des Zugriffs: 30.8.2019]

Schuster, J. (2017). Erinnern ohne Zeugen - über die Zukunft der Gedenkkultur. URL: https://www.deutscher-koordinierungsrat.de/dkr-texte-rabbiner-brandt-vorlesung-Schuster$\underline{2017}$ [Datum des Zugriffs: 30.8.2019]

Steine der Erinnerung (o.V.) (o.J.) URL https://steinedererinnerung.net/ [Datum des Zugriffs: 30.8.2019]

Uhl, H. (2003). Die Moskauer Deklaration und der Umgang Österreichs mit der Opferthese. http://www.klahrgesellschaft.at/Referate/Uhl 2003.html [Datum des Zugriffs: 30.8.2019] Uhl, H. (2010). Warum Gesellschaften sich erinnern.

URL: http://www.politischebildung.com/pdfs/32 uhl.pdf [Datum des Zugriffs: 30.8.2019]

Wallner, A.-M. (2014). Gedächtniskultur: „Erinnern ist nicht per se gut“. URL:

https://diepresse.com/home/science/3862154/Gedaechtniskultur Erinnern-ist-nicht-per-segut [Datum des Zugriffs: 30.8.2019]

Weiss, S. (2018). Friedenspreis für europäische Erinnerungskultur. URL: https://derstandard.at/2000089225669/Aleida-und-Jan-Assmann-Archaeologen-deskollektiven-Gedaechtnisses [Datum des Zugriffs: 30.8.2019] 
Welzer, H. (2011). Für eine Modernisierung der Erinnerungs- und Gedenkkultur. URL: https://www.gedenkstaettenforum.de/nc/aktuelles/einzelansicht/news/fuer eine modernisier ung der erinnerungs und gedenkkultur/ [Datum des Zugriffs: 30.8.2019]

Wolfrum, E. (2010). Erinnerungskultur und Geschichtspolitik als Forschungsfelder. Konzepte - Methoden - Themen. URL:

https://archiv.ub.uniheidelberg.de/volltextserver/13790/1/Wolfrum Erinnerungskultur und G eschichtspolitik.pdf [Datum des Zugriffs: 30.8.2019]

\section{Sonstige Quellen:}

Nationalfonds der Republik Österreich für Opfer des Nationalsozialismus (o.V.) (2019). firmeninterne Unterlage einer MitarbeiterInnenversammlung. Power Point Präsentation. Organisationsstruktur NF NEU. 14.Mai 2019. (vertraulich).

Kurztitel für den Quellenverweis im Text: Präsentation Organisationsstruktur 


\section{Anhang}

\subsection{Interviewleitfäden}

\subsubsection{Interviewleitfaden der befragten MitarbeiterInnen}

Einstiegsfragen:

- Seit wann arbeitest du beim Nationalfonds?

- Was bedeutet es für dich, beim Nationalfonds zu arbeiten - ist es nur ein Job?

Theoriegeleitete Fragen zum Nationalfonds:

- Der Nationalfonds wurde 1995 gegründet. Was waren die ersten Herausforderungen nach der Gründung des Fonds?

Leitfragen:

- Welche Aufgabenbereiche haben sich verändert in den letzten 23 Jahren seit der Gründung?

- Welche Veränderungen bedeutet es f eine Organisation, wenn sich Aufgabengebiete ändern?

- Welche Herausforderungen ergeben sich für die zukünftige Arbeit?

Theoriegeleitete Fragen zum Nationalfonds:

- Warum kam es zu der Gesetzesnovelle von 2017?

Leitfragen:

- Was verändert sich in der Organisation aufgrund der Gesetzesnovelle von 2017?

- Wird die Gesetzesnovelle in der neuen Organisationsstruktur abgebildet?

- Was bedeutet die Umstrukturierung des Nationalfonds für die MitarbeiterInnen?

- Welche Chancen und Risiken siehst du für den Nationalfonds?

- Was macht den Nationalfonds einzigartig?

Theoriegeleitete Fragen zu Erinnerungs- und Gedenkkultur:

- Stichwort Gedenkjahr 2018 - es gab sehr viele Gedenkveranstaltungen. Es gibt Kritiker, die von einer Institutionalisierung und Ritualisierung des Gedenkens durch die Überpräsenz in den Medien sprechen - womit Erinnerung und Gedenken immer abstrakter werden würden. Wie siehst du diese Kritik?

- Die amerikanische Serie „Holocaust“ aus dem Jahr 1978 gilt als ein Meilenstein in der Medienrepräsentation des Holocaust. Sie wurde heuer wieder im TV gezeigt. Hast du gewusst, dass Hannah in der Serie mitgespielt hat?

Leitfragen:

- Welchen Einfluss hatte die Serie auf die Vergangenheitsbewältigung aus heutiger Sicht?

- Wie hat sich die österreichische Erinnerungs- und Gedenkkultur in den letzten Jahren verändert?

Theoriegeleitete Fragen zu Erinnerungs- und Gedenkkultur:

- Rudolf Herz, ein Künstler und Medienforscher stellt in seinem Beitrag im Buch „Verbrechen Erinnern. Die Auseinandersetzung mit Holocaust und Völkermord“ die Frage: Wie verhält sich die Holocaust- Erinnerung zu ihrer Funktion? Kann man sagen, dass sie sich in ihrer Funktion erschöpft? Wie würdest du diese Fragen beantworten? 
Leitfragen:

- Welche Zukunft hat die Erinnerungskultur, wenn keine Zeitzeugen mehr am Leben sind?

- Wie wichtig ist Erinnerungs- und Gedenkkultur für die nächsten Generationen?

- Was kann der Nationalfonds für die österreichische Erinnerungs- und Gedenkkultur leisten?

- Was bedeutet Erinnerungs- und Gedenkkultur für dich?

- Wo siehst du den Nationalfonds in 20 Jahren?

\subsubsection{Interviewleitfaden der befragten internen Expertinnen}

Einstiegsfragen:

- Seit wann arbeitest du beim Nationalfonds?

- Seit wann leitest du den Nationalfonds? (Lessing)

- Was bedeutet es für dich, beim Nationalfonds zu arbeiten? (Ist es nur ein Job oder ist es mehr?)

- Was bedeutet es für dich, den Nationalfonds zu leiten?

Leitfragen:

- Was waren die ersten Herausforderungen nach der Gründung des Fonds?

- Welche Organisationsstruktur gab es in der ersten Zeit?

- Welche Aufgabenbereiche haben sich verändert in den letzten 24 Jahren seit der Gründung?

-

Theoriegeleitete Fragen zum Nationalfonds:

- Warum kam es zu der Gesetzesnovelle von 2017?

Leitfragen:

- Was verändert sich in der Organisation aufgrund der Gesetzesnovelle?

- Was bedeuten diese Veränderungen für die zukünftige Arbeit des Nationalfonds?

- Wird die Gesetzesnovelle in der neuen Organisationsstruktur abgebildet?

- Was gilt es in der Umstrukturierung des Nationalfonds zu bedenken?

- Was bedeutet die Umstrukturierung des Nationalfonds für die MitarbeiterInnen?

- Welche Chancen und Risiken siehst du für den Nationalfonds?

- Was macht den Nationalfonds einzigartig?

Theoriegeleitete Fragen zu Erinnerungs- und Gedenkkultur:

- Die amerikanische Serie „Holocaust" aus dem Jahr 1978 gilt als ein Meilenstein in der Medienrepräsentation des Holocaust. Sie wird heuer wieder im TV gezeigt. Du hast in der Serie damals mitgespielt. Wie war das damals für dich? (Lessing)

- Hast du gewusst, dass Hannah in der Serie mitgespielt hat? (Meissner)

- Welchen Einfluss hatte die Serie auf die Vergangenheitsbewältigung aus heutiger Sicht?

Leitfragen:

- Wie hat sich die österreichische Erinnerungs- und Gedenkkultur in den letzten Jahren verändert?

- Stichwort Gedenkjahr 2018 - es gab sehr viele Gedenkveranstaltungen. Es gibt Kritiker, die von einer Institutionalisierung und Ritualisierung des Gedenkens durch die Überpräsenz in den Medien sprechen - womit Erinnerung und Gedenken immer abstrakter werden würden. Wie siehst du diese Kritik? 
Theoriegeleitete Fragen zu Erinnerungs- und Gedenkkultur:

- Rudolf Herz, ein Künstler und Medienforscher stellt in seinem Beitrag im Buch „Verbrechen Erinnern. Die Auseinandersetzung mit Holocaust und Völkermord“ die Frage: Wie verhält sich die Holocaust- Erinnerung zu ihrer Funktion? Kann man sagen, dass sie sich in ihrer Funktion erschöpft? Wie würdest du diese Fragen beantworten?

Leitfragen:

- Welche Zukunft hat die Erinnerungskultur, wenn keine Zeitzeugen mehr am Leben sind?

- Wie wichtig ist Erinnerungskultur für die nächsten Generationen?

- Was kann der Nationalfonds für die österreichische Erinnerungs- und Gedenkkultur leisten?

- Welche Gefahren siehst du für den Erhalt der Erinnerungskultur in den nächsten Jahrzehnten?

- Was bedeutet Erinnerungskultur für dich?

- Wo siehst du den Nationalfonds in 20 Jahren?

\subsubsection{Interviewleitfaden der befragten externen Expertin (Dr. Janistyn-}

\section{Novák)}

Theoriegeleitete Fragen zum Nationalfonds:

- Wie haben Sie die Gründung des Nationalfonds erlebt?

Leitfragen:

- Welche Herausforderungen gab es für die Arbeit des Nationalfonds in der ersten Zeit nach der Gründung?

- Welche Organisationsstruktur gab es in der ersten Zeit?

Theoriegeleitete Fragen zum Nationalfonds:

- Was bedeutet die Gesetzesnovelle von 2017 für die zukünftige Arbeit des Nationalfonds?

Leitfragen:

- Welche Herausforderungen ergeben sich für die zukünftige Arbeit des Nationalfonds auch aufgrund der letzten Gesetzesnovelle?

- Was kann der Nationalfonds für die österreichische Erinnerungs- und Gedenkkultur leisten?

- Sehen Sie den Nationalfonds als Teil der Landschaft der österreichischen Erinnerungskultur? Wenn ja, wodurch?

- Welche Bedeutung hat der Nationalfonds im 21. Jahrhundert?

- Wo sehen Sie den Nationalfonds in 20 Jahren?

Theoriegeleitete Fragen zu Erinnerungs- und Gedenkkultur:

- Stichwort Gedenkjahr 2018 - es gab sehr viele Gedenkveranstaltungen. Es gibt Kritiker, die von einer Institutionalisierung und Ritualisierung des Gedenkens durch die Überpräsenz in den Medien sprechen - womit Erinnerung und Gedenken immer abstrakter werden würden. Wie sehen Sie diese Kritik?

- Wie hat sich die österreichische Erinnerungs- und Gedenkkultur in den letzten Jahren verändert? 
Leitfragen:

- Welche Gefahren sehen Sie für den Erhalt der Erinnerungskultur in den nächsten Jahrzehnten?

- Wie wichtig ist Erinnerungskultur für die nächsten Generationen?

- Was bedeutet Erinnerungskultur für Sie?

\subsubsection{Interviewleitfaden der befragten externen Expertin (Mag. Maschke)}

Theoriegeleitete Fragen zum Nationalfonds:

- Wie haben Sie die Gründung des Nationalfonds erlebt?

Theoriegeleitete Fragen zum Nationalfonds:

- Haben Sie von der Gesetzesnovelle 2017 des Nationalfonds gehört?

- Was bedeutet die Gesetzesnovelle von 2017 für die zukünftige Arbeit des Nationalfonds?

Leitfragen:

- Welche Herausforderungen ergeben sich für die zukünftige Arbeit des Nationalfonds auch aufgrund der letzten Gesetzesnovelle?

- Was kann der Nationalfonds für die österreichische Erinnerungs- und Gedenkkultur leisten?

- Sehen Sie den Nationalfonds als Teil der Landschaft der österreichischen Erinnerungskultur? Wenn ja, wodurch?

- Wo sehen Sie den Nationalfonds in 20 Jahren?

Theoriegeleitete Fragen zu Erinnerungs- und Gedenkkultur:

- Stichwort Gedenkjahr 2018 - es gab sehr viele Gedenkveranstaltungen. Es gibt Kritiker, die von einer Institutionalisierung und Ritualisierung des Gedenkens durch die Überpräsenz in den Medien sprechen - womit Erinnerung und Gedenken immer abstrakter werden würden. Wie sehen Sie diese Kritik?

- Wie hat sich die österreichische Erinnerungs- und Gedenkkultur in den letzten Jahren verändert?

- Welche Zukunft hat die Erinnerungskultur, wenn keine Zeitzeugen mehr am Leben sind?

Leitfragen:

- Welche Gefahren sehen Sie für den Erhalt der Erinnerungskultur in den nächsten Jahrzehnten?

- Wie wichtig ist Erinnerungskultur für die nächsten Generationen?

- Wie kann Erinnerungskultur der jungen Generation bzw. den nächsten Generationen vermittelt werden?

Theoriegeleitete Fragen zu Erinnerungs- und Gedenkkultur:

- Die amerikanische Serie „Holocaust" aus dem Jahr 1978 gilt als ein Meilenstein in der Medienrepräsentation des Holocaust. Sie wurde heuer wieder im TV gezeigt.

Welchen Einfluss hatte die Serie auf die Vergangenheitsbewältigung aus heutiger Sicht?

Theoriegeleitete Fragen zu Erinnerungs- und Gedenkkultur:

- Rudolf Herz, ein Künstler und Medienforscher stellt in seinem Beitrag im Buch "Verbrechen Erinnern. Die Auseinandersetzung mit Holocaust und Völkermord“ die Frage: Wie verhält sich die Holocaust- Erinnerung zu ihrer Funktion? Kann man sagen, dass sie sich in ihrer Funktion erschöpft? Wie würden Sie diese Fragen beantworten? 


\subsubsection{Interviewleitfaden der befragten externen Expertin (Dr. Uhl)}

Theoriegeleitete Fragen zu Erinnerungs- und Gedenkkultur:

- Die amerikanische Serie „Holocaust“ aus dem Jahr 1978 gilt als ein Meilenstein in der Medienrepräsentation des Holocaust. Sie wird heuer wieder im TV gezeigt. Welchen Einfluss hatte die Serie auf die Vergangenheitsbewältigung aus heutiger Sicht?

\section{Leitfragen:}

- Wie hat sich die österreichische Erinnerungs- und Gedenkkultur in den letzten Jahren verändert?

- Stichwort Gedenkjahr 2018 - es gab sehr viele Gedenkveranstaltungen. Es gibt Kritiker, die von einer Institutionalisierung und Ritualisierung des Gedenkens durch die Überpräsenz in den Medien sprechen - womit Erinnerung und Gedenken immer abstrakter werden würden. Wie sehen Sie diese Kritik?

- Welche Zukunft hat die Erinnerungskultur, wenn keine Zeitzeugen mehr am Leben sind?

- Wie wichtig ist Erinnerungskultur für die nächsten Generationen?

- Wie kann Erinnerungskultur der jungen Generation bzw den nächsten Generationen vermittelt werden?

Theoriegeleitete Fragen zu Erinnerungs- und Gedenkkultur:

- Rudolf Herz, ein Künstler und Medienforscher stellt in seinem Beitrag im Buch „Verbrechen Erinnern. Die Auseinandersetzung mit Holocaust und Völkermord" die Frage: Wie verhält sich die Holocaust- Erinnerung zu ihrer Funktion? Kann man sagen, dass sie sich in ihrer Funktion erschöpft? Wie würden Sie diese Fragen beantworten?

Leitfragen:

- Welche Gefahren sehen Sie für den Erhalt der Erinnerungskultur in den nächsten Jahrzehnten?

Theoriegeleitete Fragen zum Nationalfonds:

- Wie haben Sie die Gründung des Nationalfonds erlebt?

Leitfragen:

- Was kann der Nationalfonds für die österreichische Erinnerungs- und Gedenkkultur leisten?

- Sehen Sie den Nationalfonds als Teil der Landschaft der österreichischen Erinnerungskultur? Wenn ja, wodurch?

- Welche Herausforderungen ergeben sich für die zukünftige Arbeit des Nationalfonds - auch aufgrund der letzten Gesetzesnovelle von 2017? (evtl. erklären)

- Welche Bedeutung hat der Nationalfonds im 21. Jahrhundert?

- Wo sehen Sie den Nationalfonds in 20 Jahren? 


\subsection{Extraktionstabellen}

\begin{tabular}{|c|c|c|c|c|c|c|c|}
\hline \begin{tabular}{|l|}
$\begin{array}{l}\text { Kate- } \\
\text { gorien }\end{array}$ \\
\end{tabular} & \begin{tabular}{|l|}
$\begin{array}{l}\text { Sub- } \\
\text { kategorien }\end{array}$ \\
\end{tabular} & Int.1 & Int.2 & Int.3 & Int.4 & Int.5 & Int.6 \\
\hline \multirow[t]{5}{*}{ Kat.1 } & Subkat.1.1 & $\begin{array}{l}\text { S.2, Z.47-48 } \\
\text { S.2, Z.42-43 } \\
\text { S.3, Z.75-78 }\end{array}$ & S.2, Z.66-71 & $\begin{array}{l}\text { S.1, Z.27 } \\
\text { S.1, Z.26-30 } \\
\text { S.4,Z.120-121 } \\
\text { S.4,Z.119-127 }\end{array}$ & & & $\begin{array}{l}\text { S.2, Z.43-44 } \\
\text { S.2, Z.59-65 }\end{array}$ \\
\hline & Subkat.1.2 & $\begin{array}{l}\text { S.3-4,Z.110-118 } \\
\text { S.4,Z.126-131 }\end{array}$ & S.2-3, Z.71-83 & $\begin{array}{l}\text { S.4, Z.133-134 } \\
\text { S.4, Z.135-147 } \\
\text { S.5, Z.170-177 } \\
\text { S.5, Z.181-183 }\end{array}$ & & S.3, Z.75-79 & $\begin{array}{l}\text { S.4, Z.129-137 } \\
\text { S.6, Z.201-209 }\end{array}$ \\
\hline & Subkat.1.3 & S.1, Z.21-24 & S.1-2, Z.35-40 & S.1, Z.19-21 & S.1, Z.13-14 & S.1, Z.12-17 & S.1, Z.15-25 \\
\hline & Subkat.1.4 & S.6, Z.200-203 & $\begin{array}{l}\text { S.7, Z.247 } \\
\text { S.7-8,Z.258-262 }\end{array}$ & $\begin{array}{l}\text { S.9, Z.305-307 } \\
\text { S.10,Z.332-335 } \\
\text { S.10,Z.339-341 }\end{array}$ & S.4, Z.141-146 & $\begin{array}{l}\text { S.4-5, Z.146-152 } \\
\text { S.5, Z.156-158 }\end{array}$ & \begin{tabular}{|l|} 
S.7, Z.226-230 \\
S.7, Z.235-236 \\
S.7, Z.240-244 \\
S.7, Z.249
\end{tabular} \\
\hline & Subkat.1.5 & $\begin{array}{l}\text { S.3, Z.87-88 } \\
\text { S.5, Z.154-161 }\end{array}$ & $\begin{array}{l}\text { S.3, Z.103-107 } \\
\text { S.3-4,Z.109-116 } \\
\text { S.4, Z.130-132 } \\
\text { S.4, Z.142-143 } \\
\text { S.4, Z.140-149 } \\
\text { S.16, Z.575-580 } \\
\end{array}$ & $\begin{array}{l}\text { S.2, Z.49-55 } \\
\text { S.3, Z.75-90 } \\
\text { S.3, Z.90-92 }\end{array}$ & & $\begin{array}{l}\text { S.1-2, Z.37-42 } \\
\text { S.2, Z.47-51 }\end{array}$ & S.3, Z.91-93 \\
\hline \multirow[t]{2}{*}{ Kat.2 } & Subkat.2.1 & & S.7-8,Z.258-263 & & & & \\
\hline & Subkat.2.2 & & $\begin{array}{l}\text { S.5, Z.164-165 } \\
\text { S.5, Z.179-180 } \\
\text { S.6, Z.186-187 }\end{array}$ & \begin{tabular}{|l|} 
S.6, Z.195-198 \\
S.6, Z.198-203 \\
S.7, Z.244-247 \\
S.15-16,Z.546- \\
550 \\
\end{tabular} & S.2, Z.69-74 & $\begin{array}{l}\text { S.2, Z.67-71 } \\
\text { S.3, Z.75-79 } \\
\text { S.3, Z.84-86 } \\
\text { S.3, Z.91-102 }\end{array}$ & S.4-5, Z.143-165 \\
\hline \multirow[t]{2}{*}{ Kat.3 } & Subkat.3.1 & S.5, Z.183-185 & S.6, Z.194-202 & \begin{tabular}{|l|} 
S.3, Z.102-111 \\
S.4, Z.135-147 \\
S.5, Z.151-157 \\
S.5, Z.170-177 \\
S.5, Z.181-183 \\
S.7, Z.248-249 \\
S.7, Z.247-257 \\
S.7-8,Z.257-272 \\
S.8, Z.277 \\
S.8, Z.278-288 \\
\end{tabular} & $\begin{array}{l}\text { S.3, Z.82-88 } \\
\text { S.3, Z.96-100 } \\
\text { S.3, Z.109-112 } \\
\text { S.3-4,Z.112-119 }\end{array}$ & $\begin{array}{l}\text { S.3-4, Z.106-112 } \\
\text { S.4, Z.116-119 } \\
\text { S.4, Z.139-142 }\end{array}$ & $\begin{array}{l}\text { S.6, Z.191-195 } \\
\text { S.6, Z.200-209 }\end{array}$ \\
\hline & Subkat.3.2 & & & S.7, Z.244-247 & & & S.5-6, Z.182-187 \\
\hline \multirow[t]{2}{*}{ Kat.4 } & Subkat.4.1 & $\begin{array}{l}\text { S.2, Z.59-65 } \\
\text { S.3, Z.78-81 }\end{array}$ & & \begin{tabular}{|l} 
S.1-2, Z.34-39 \\
S.2, Z.39-42
\end{tabular} & $\begin{array}{l}\text { S.1, Z.28-32 } \\
\text { S.1, Z.36-37 } \\
\text { S.1, Z.31-33 }\end{array}$ & & $\begin{array}{l}\text { S.1-2,Z.39-41 } \\
\text { S.1-2, Z.37-44 } \\
\text { S.2, Z.48-53 } \\
\text { S.1, Z.31-33 } \\
\text { S.2-3, Z.71-82 } \\
\text { S.3, Z.79 } \\
\text { S.2-3,Z.80-82 }\end{array}$ \\
\hline & Subkat.4.2 & S.6, Z.189-190 & S.5, Z.164-170 & & \begin{tabular}{|l|} 
S.2, Z.48-51 \\
S.3, Z.88-94 \\
S.4, Z.126-131 \\
\end{tabular} & S.2, Z.59-61 & \begin{tabular}{|l|} 
S.4, Z.112-118 \\
S.3, Z.100-103 \\
S.3, Z.107-108
\end{tabular} \\
\hline \multirow[t]{3}{*}{ Kat.5 } & Subkat.5.1 & S.9, Z.310-312 & \begin{tabular}{|l} 
S.16, Z.565-570 \\
S.16, Z.582-587 \\
S.16-17,Z.591- \\
600 \\
S.18-19,Z.660- \\
669 \\
\end{tabular} & \begin{tabular}{|l} 
S.13, Z.457-465 \\
S.13-14,Z.470- \\
476 \\
S.17, Z.596-597
\end{tabular} & $\begin{array}{l}\text { S.1-2, Z.37-43 } \\
\text { S.8, Z.292-295 } \\
\text { S.9, Z.299-308 }\end{array}$ & $\begin{array}{l}\text { S.8, Z.286-293 } \\
\text { S.5, Z.177 }\end{array}$ & S.11, Z.384-385 \\
\hline & Subkat.5.2 & S.6, Z.194-196 & & \begin{tabular}{|l|} 
S.9, Z.305-306 \\
S.8, Z.294 \\
S.8-9, Z.292-305 \\
\end{tabular} & & $\begin{array}{l}\text { S.4, Z.130-131 } \\
\text { S.4, Z.136-138 }\end{array}$ & S.5, Z.170-172 \\
\hline & Subkat.5.3 & & S.6-7,Z.215-229 & & & S.4, Z.125-129 & \\
\hline
\end{tabular}




\begin{tabular}{|c|c|c|c|c|c|c|c|}
\hline \begin{tabular}{|l|}
$\begin{array}{l}\text { Kate- } \\
\text { gorien }\end{array}$ \\
\end{tabular} & \begin{tabular}{|l} 
Sub- \\
kategorien
\end{tabular} & | Int.1 & Int.2 & Int.3 & Int.4 & Int.5 & Int.6 \\
\hline \multicolumn{8}{|l|}{ Kat.6 } \\
\hline & Subkat.6.1 & $\begin{array}{l}\text { S.7, Z.245 } \\
\text { S.7, Z.251-253 }\end{array}$ & $\begin{array}{l}\text { S.10-11,Z.369- } \\
372 \\
\text { S.11,Z.393-396 } \\
\text { S.15,Z.527-531 }\end{array}$ & $\begin{array}{l}\text { S.12, Z.415-416 } \\
\text { S.12, Z.420-421 } \\
\text { S.12-13,Z.424- } \\
440 \\
\text { S.10, Z.354-355 } \\
\end{array}$ & & & S.10,Z.353-355 \\
\hline & Subkat.6.2 & & & & & & \\
\hline \multirow[t]{4}{*}{ Kat.7 } & Subkat.7.1 & S.6, Z.213-215 & $\begin{array}{l}\text { S.8, Z.273-284 } \\
\text { S.8, Z.284-287 } \\
\text { S.8, Z.287-291 } \\
\text { S.8, Z.291-302 }\end{array}$ & \begin{tabular}{|l|} 
S.10,Z.347-353 \\
S.10-11,Z.354- \\
369 \\
S.18,Z.624-627 \\
S.18,Z.632-638 \\
S.19,Z.662-666 \\
\end{tabular} & & $\begin{array}{l}\text { S.5, Z.167-172 } \\
\text { S.5, Z.177-182 }\end{array}$ & S.8, Z.260-267 \\
\hline & Subkat.7.2 & & $\begin{array}{l}\text { S.12, Z.410-414 } \\
\text { S.13, Z.464-467 }\end{array}$ & S.13, Z.449-457 & $\begin{array}{l}\text { S.5, Z.175-186 } \\
\text { S.6, Z.194-197 }\end{array}$ & S.6-7, Z.217-228 & S.10, Z.336-345 \\
\hline & Subkat.7.3 & S.8, Z.278-281 & $\begin{array}{l}\text { S.15, Z.550-552 } \\
\text { S.18, Z.644-648 }\end{array}$ & $\begin{array}{l}\text { S.15-16, Z.541- } \\
556\end{array}$ & S.7-8, Z.258-268 & $\begin{array}{l}\text { S.7, Z.253-261 } \\
\text { S.8, Z.266-272 } \\
\end{array}$ & \begin{tabular}{|l|} 
S.9, Z.306-307 \\
S.9, Z.308-309 \\
\end{tabular} \\
\hline & Subkat.7.4 & S.9, Z.301-302 & S.17, Z.605-606 & S.16, Z.560-569 & S.8, Z.272-278 & & \\
\hline Kat.8 & & S.7, Z.225-230 & \begin{tabular}{|l|} 
S.9, Z.320-323 \\
S.9-10,Z.327- \\
338 \\
S.10, Z.349-351 \\
S.10, Z.351-361 \\
\end{tabular} & $\begin{array}{l}\text { S.11, Z.377-384 } \\
\text { S.11, Z.384-401 }\end{array}$ & S.5, Z.167-175 & S.6, Z.190-202 & S.9, Z.320-327 \\
\hline \multirow[t]{3}{*}{ Kat.9 } & Subkat.9.1 & & & & & & \\
\hline & Subkat.9.2 & & $\begin{array}{l}\text { S.14-15, Z.518- } \\
524\end{array}$ & & & & \\
\hline & Subkat.9.3 & S.8, Z.272-273 & $\begin{array}{l}\text { S.15, Z.527-531 } \\
\text { S.12, Z.430 }\end{array}$ & $\begin{array}{l}\text { S.14, Z.481-491 } \\
\text { S.14, Z.504-505 } \\
\text { S.18-19,Z.652- } \\
671 \\
\text { S.16,Z.562-564 } \\
\text { S.16-17, Z.576- } \\
587\end{array}$ & & $\begin{array}{l}\text { S.7, Z.233-240 } \\
\text { S.7, Z.244 }\end{array}$ & $\begin{array}{l}\text { S.8, Z.272-276 } \\
\text { S.8, Z.286-289 }\end{array}$ \\
\hline
\end{tabular}




\begin{tabular}{|c|c|c|c|c|c|c|}
\hline Kategorien & $\begin{array}{l}\text { Sub- } \\
\text { kategorien }\end{array}$ & Mag. Maschke & Dr. Uhl & Dr. Janistyn & Mag. Lessing & Dr. Meissner \\
\hline \multirow[t]{5}{*}{ Kat.1 } & Subkat.1.1 & & S.5, Z.181-186 & \begin{tabular}{|l} 
S.2, Z.49-53 \\
S.17, Z.612-616 \\
S.3, Z.79-81 \\
S.4-5,Z.145-152 \\
S.5, Z.152-153 \\
S.3, Z.77-79 \\
S.5, Z.177-180 \\
S.3, Z.100-103 \\
S.5, Z.180-184 \\
S.7, Z.246-249 \\
S.7, Z.249-251 \\
S.17, Z.591-592
\end{tabular} & $\begin{array}{l}\text { S.2-3, Z.73-81 } \\
\text { S.3, Z.81-84 } \\
\text { S.3, Z. 88-89 } \\
\text { S.3, Z.88-92 } \\
\text { S.5, Z.157-158 }\end{array}$ & \begin{tabular}{|l|} 
S.2, Z.46-48 \\
S.2, Z.50-51 \\
S.2, Z.53-60 \\
S.2, Z.57-58 \\
S.2, Z.60-66 \\
S.2. Z.66-70 \\
S.3, Z.75-81 \\
S.4, Z.140 \\
S.5, Z.151-154 \\
S.5,Z.154 \\
S.5, Z.154-157 \\
S.5, Z.163-173 \\
S.9, Z.305-306 \\
S.9, Z. $306-310$ \\
\end{tabular} \\
\hline & Subkat.1.2 & S.4, Z.115-120 & & \begin{tabular}{|l} 
S.7, Z.233-234 \\
S.7, Z.234-236 \\
S.7. Z.241 \\
S.16, Z.583-588 \\
\end{tabular} & $\begin{array}{l}\text { S.5, Z.169-176 } \\
\text { S.5-6, Z.181-184 }\end{array}$ & S.12, Z.418-430 \\
\hline & Subkat.1.3 & & & & $\begin{array}{l}\text { S.1, Z.9-11 } \\
\text { S.1, Z.15 } \\
\end{array}$ & \begin{tabular}{|l|} 
S.1, Z.26-30 \\
S.2, Z.44-53
\end{tabular} \\
\hline & Subkat.1.4 & & & \begin{tabular}{|l|} 
S.2, Z.42-48 \\
S.4,Z.128-131 \\
S.4,Z.136-138 \\
\end{tabular} & \begin{tabular}{|l|} 
S.6, Z.189-200 \\
S.8, Z.264-268 \\
S.8, Z.268-277 \\
\end{tabular} & $\begin{array}{l}\text { S.5-6, Z.177-190 } \\
\text { S.19, Z.680-686 } \\
\text { S.19, Z.686-700 } \\
\end{array}$ \\
\hline & Subkat.1.5 & & & & $\begin{array}{l}\text { S.3, Z.98-100 } \\
\text { S.3, Z.100-104 } \\
\text { S.3-4, Z.104-112 } \\
\text { S.4, Z.112-116 } \\
\text { S.6, Z.211-221 }\end{array}$ & \begin{tabular}{|l|} 
S.9, Z.319 \\
S.9, Z.314-315 \\
S.9, Z.304-310 \\
S.9, Z.310-319 \\
S.9, Z.326-329 \\
S.10, Z.334-341 \\
S.9-10,Z.329-344 \\
S.10, Z.345-347 \\
S.11, Z.402 \\
S.11, Z.397-405 \\
\end{tabular} \\
\hline \multirow[t]{2}{*}{ Kat.2 } & Subkat.2.1 & & & \begin{tabular}{|l|} 
S.2, Z.59-65 \\
S.2, Z.65-69 \\
\end{tabular} & S.7, Z.238-240 & \begin{tabular}{|l|} 
S.3, Z.86 \\
S.3, Z.91-94
\end{tabular} \\
\hline & Subkat.2.2 & S.3, Z.91-100 & & $\begin{array}{l}\text { S.1, Z.28-30 } \\
\text { S.9, Z.312-317 } \\
\text { S.19, Z.678-681 }\end{array}$ & S.4, Z.132-135 & \begin{tabular}{|l|} 
S.10, Z.354-361 \\
S.11, Z.370-376 \\
S.11, Z.382-393 \\
S.14, Z.500-503
\end{tabular} \\
\hline \multirow[t]{2}{*}{ Kat.3 } & Subkat.3.1 & & & & $\begin{array}{l}\text { S.5, Z.169-176 } \\
\text { S.5, Z.159-165 } \\
\text { S.5-6, Z.183-184 } \\
\text { S.6, Z.189-194 } \\
\text { S.6, Z.194-200 } \\
\text { S.6-7, Z.207-221 }\end{array}$ & $\begin{array}{l}\text { S.14-15,Z.517-528 } \\
\text { S.15, Z.532-544 } \\
\text { S.16, Z.558-567 } \\
\text { S.16, Z.567-572 } \\
\text { S.13, Z.475-477 } \\
\text { S.13-14,Z.477-490 } \\
\text { S.14, Z.491 } \\
\text { S.12-13,Z.441-446 } \\
\text { S.16-17,Z.585-593 } \\
\text { S.17, Z.601-618 }\end{array}$ \\
\hline & Subkat.3.2 & S.3, Z.91-100 & & & $\begin{array}{l}\text { S.4, Z.140-146 } \\
\text { S.4-5, Z.147-149 } \\
\text { S.5, Z.154-165 }\end{array}$ & $\begin{array}{l}\text { S.12-13,Z.441-446 } \\
\text { S.13,Z.458-461 } \\
\text { S.14,Z.502-503 }\end{array}$ \\
\hline
\end{tabular}




\begin{tabular}{|c|c|c|c|c|c|c|}
\hline Kategorien & \begin{tabular}{|l} 
Sub- \\
kategorien
\end{tabular} & Mag. Maschke & Dr. Uhl & Dr. Janistyn & Mag. Lessing & Dr. Meissner \\
\hline \multirow[t]{2}{*}{ Kat.4 } & Subkat.4.1 & & & \begin{tabular}{|l} 
S.2, Z.69-71 \\
S.2, Z.65-69 \\
S.3, Z.79-81 \\
S.3, Z.100-104 \\
S.4, Z.143-147 \\
S.5, Z.154-162 \\
S.5, Z.163-164 \\
S.5, Z.175-177 \\
S.6, Z.199-201 \\
S.6, Z.185-192 \\
S.6, Z.192-194 \\
S.6, Z.198-207 \\
S.6, Z.212-214 \\
S.7, Z.217-220 \\
S.7, Z.224-234 \\
S.7, Z.231-234 \\
S.7, Z.251-258 \\
S.7-8,Z.258-264 \\
S.8,Z.286-287
\end{tabular} & $\begin{array}{l}\text { S.2, Z.60-63 } \\
\text { S.2, Z.63-69 } \\
\text { S.2, Z.45-52 } \\
\text { S.1, Z. 29-35 } \\
\text { S.1-2, Z.36-41 }\end{array}$ & $\begin{array}{l}\text { S.7, Z.245-249 } \\
\text { S.7, Z.249-252 } \\
\text { S.8, Z.263-273 } \\
\text { S.6, Z.214-220 } \\
\text { S.7, Z.225-226 } \\
\text { S.6-7, Z.220-224 } \\
\text { S.4, Z.113-124 } \\
\text { S.4, Z.124-131 } \\
\text { S.7, Z.230-238 } \\
\text { S.7., Z.249-252 } \\
\text { S.8, Z.276-281 } \\
\text { S.8., Z.285-289 }\end{array}$ \\
\hline & Subkat.4.2 & & S.8, Z.282-284 & S.18, Z.654-659 & S.7, Z.247-256 & $\begin{array}{l}\text { S.10, Z.344-347 } \\
\text { S.13, Z.475-477 }\end{array}$ \\
\hline \multirow[t]{3}{*}{ Kat.5 } & Subkat.5.1 & $\begin{array}{l}\text { S.9, Z.321-331 } \\
\text { S.3, Z.108-110 } \\
\text { S.10,Z.332-334 }\end{array}$ & $\begin{array}{l}\text { S.7, Z.230-233 } \\
\text { S.8, Z.282-284 } \\
\text { S.8, Z.288-289 }\end{array}$ & \begin{tabular}{|l|} 
S.9, Z.317-321 \\
S.11, Z.369-379 \\
S.12, Z.426-434 \\
S.11, Z.382-386 \\
S.9, Z.321-328 \\
S.9, Z.328-331 \\
S.9, Z.333-338 \\
S.14, Z.496-504 \\
S.10, Z.332-338 \\
S.12, Z.426-434 \\
S.15, Z.523-530 \\
S.15, Z.535-540 \\
S.15, Z.542-550 \\
S.13, Z.470-474
\end{tabular} & $\begin{array}{l}\text { S.13, Z.466-470 } \\
\text { S.6-7, Z.215-221 } \\
\text { S.14, Z.478 } \\
\text { S.16, Z.558-566 } \\
\text { S.16, Z.581-583 }\end{array}$ & $\begin{array}{l}\text { S.9-10,Z.329-344 } \\
\text { S.27, Z.962-974 } \\
\text { S.27, Z.979 } \\
\text { S.23-24,Z.847-859 } \\
\text { S.24-25,Z.882-887 }\end{array}$ \\
\hline & Subkat.5.2 & & & & $\begin{array}{l}\text { S.7, Z.226-227 } \\
\text { S.7, Z.230-236 }\end{array}$ & \begin{tabular}{|l|} 
S.18, Z.637-644 \\
S.18, Z.644-652 \\
S.19, Z.666-676 \\
\end{tabular} \\
\hline & Subkat.5.3 & & & & S.7, Z.252 & S.18-19,Z.654-666 \\
\hline \multirow[t]{3}{*}{ Kat.6 } & & & & S.1, Z.48-53 & S.1-2, Z.36-38 & S.21, Z.757-763 \\
\hline & Subkat.6.1 & & $\begin{array}{l}\text { S.3, Z.99 } \\
\text { S.3, Z.102 }\end{array}$ & & \begin{tabular}{|l|} 
S.8-9, Z. $285-305$ \\
S.8-9, Z.293-295 \\
S.9, Z.296-302 \\
S.9, Z.314-323
\end{tabular} & $\begin{array}{l}\text { S.20, Z.729-732 } \\
\text { S.21, Z.744-750 }\end{array}$ \\
\hline & Subkat.6.2 & & S.4, Z.116-125 & & S.2, Z.39-41 & \begin{tabular}{|l|} 
S.21, Z.764-766 \\
S.22,Z.777-781
\end{tabular} \\
\hline
\end{tabular}




\begin{tabular}{|c|c|c|c|c|c|c|}
\hline \begin{tabular}{|l|} 
Kate- \\
gorien
\end{tabular} & \begin{tabular}{|l|} 
Sub- \\
kategorien
\end{tabular} & Mag. Maschke & Dr. Uhl & Dr. Janistyn & Mag. Lessing & Dr. Meissner \\
\hline \multirow[t]{4}{*}{ Kat.7 } & Subkat.7.1 & S.7, Z.238-241 & \begin{tabular}{|l|} 
S.9, Z.309 \\
S.11,Z.398-399 \\
\end{tabular} & S.6, Z.214-217 & \begin{tabular}{|l|} 
S.8, Z.285-286 \\
S.9-10, Z.328-337
\end{tabular} & S.21, Z.757-780 \\
\hline & Subkat.7.2 & & & & & \\
\hline & Subkat.7.3 & & $\begin{array}{l}\text { S.6, Z.191-195 } \\
\text { S.6, Z.206-207 }\end{array}$ & $\begin{array}{l}\text { S.14,Z.480-481 } \\
\text { S.16,Z.585 } \\
\text { S.8,Z.273-277 }\end{array}$ & $\begin{array}{l}\text { S.15,Z.520-521 } \\
\text { S.15, Z.521-522 } \\
\text { S.15, Z.522-534 }\end{array}$ & $\begin{array}{l}\text { S.22, Z.777-793 } \\
\text { S.25, Z.892-895 } \\
\text { S.22, Z.798-807 } \\
\text { S.24, Z.873-878 } \\
\text { S.24-25,Z.882-885 }\end{array}$ \\
\hline & Subkat.7.4 & & & & S.15-16,Z.542-553 & $\begin{array}{l}\text { S.26, Z.923-927 } \\
\text { S.26, Z.927-938 }\end{array}$ \\
\hline Kat.8 & & $\begin{array}{l}\text { S.4, Z.130-134 } \\
\text { S.4-5,Z.143-166 }\end{array}$ & $\begin{array}{l}\text { S.9,Z.323-325 } \\
\text { S.12,Z.418-424 }\end{array}$ & S.10, Z.348-357 & $\begin{array}{l}\text { S.12, Z.407-415 } \\
\text { S.12, Z.415-419 } \\
\text { S.12, Z.420-426 }\end{array}$ & S.23, Z.817-832 \\
\hline \multirow[t]{3}{*}{ Kat.9 } & Subkat.9.1 & S.7, Z.245-253 & \begin{tabular}{|l|} 
S.12,Z.431-432 \\
S.12,Z.432-433 \\
\end{tabular} & & \begin{tabular}{|l} 
S.10, Z.343-344 \\
S.11, Z.376-382 \\
\end{tabular} & S. $25, Z .900-910$ \\
\hline & Subkat.9.2 & & $\begin{array}{l}\text { S.7,Z.238 } \\
\text { S.7-8,Z.248-264 }\end{array}$ & & & \\
\hline & Subkat.9.3 & $\begin{array}{l}\text { S.5, Z.153-163 } \\
\text { S.8-9, Z.291-297 } \\
\text { S.5-6, Z.182-188 } \\
\text { S.6, Z.198-205 }\end{array}$ & S.10, Z.345-351 & $\begin{array}{l}\text { S.12, Z.436-441 } \\
\text { S.15, Z.523-530 } \\
\text { S.16, Z.758-583 }\end{array}$ & \begin{tabular}{|l} 
S.12-13,Z.434-437 \\
S.13,Z.447-458 \\
S.13, Z.458-466 \\
S.10-11,Z.365-373 \\
S.11, Z.376-382 \\
S.10, Z.344-361 \\
S.14, Z.483-489 \\
S.14, Z.Z.493-497 \\
S.11, Z.382-393
\end{tabular} & $\begin{array}{l}\text { S.23, Z.837-847 } \\
\text { S.24, Z.863-864 }\end{array}$ \\
\hline
\end{tabular}




\subsection{Abbildungsverzeichnis}

Abb.1: Die Organe des Nationalfonds der Republik Österreich für Opfer des Nationalsozialismus, des Allgemeinen Entschädigungsfonds für Opfer des Nationalsozialismus und des Fonds zur Instandsetzung der jüdischen Friedhöfe in Österreich.

Abb.2: Die neue Organisationsstruktur im Überblick in der Übergangsphase bis 31.12.2019. (Quelle: Präsentation Organisationsstruktur, 2019)

Abb.3: Die neue Organisationsstruktur im Überblick ab 1.1.2020. (Quelle: Präsentation Organisationsstruktur, 2019) S.106 


\section{Eigenständigkeitserklärung}

Ich erkläre an Eides statt, dass ich die vorliegende Masterarbeit eigenständig und ohne fremde Hilfe verfasst, andere als die angegebenen Quellen nicht benutzt und die den Quellen wörtlich oder inhaltlich entnommenen Stellen als solche kenntlich gemacht habe.

Die Masterarbeit wurde bisher in gleicher oder ähnlicher Form keiner anderen inländischen oder ausländischen Prüfungsbehörde vorgelegt und auch noch nicht veröffentlicht.

Die vorliegende Fassung entspricht der eingereichten elektronischen Version.

$$
3.9 .2019
$$

Wien, September 2019

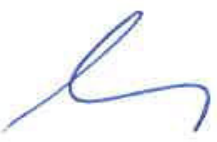

(Stephanie Mayerhofer) 